Florida International University FIU Digital Commons

$8-1-2001$

\title{
Toward a unified theory of task-oriented and relationship-oriented leader behavior: a multi- country generalizability study
}

Ellen Antoinette Drost

Florida International University

DOI: $10.25148 /$ etd.FI15101228

Follow this and additional works at: https://digitalcommons.fiu.edu/etd

Part of the Organizational Behavior and Theory Commons

\section{Recommended Citation}

Drost, Ellen Antoinette, "Toward a unified theory of task-oriented and relationship-oriented leader behavior: a multi-country generalizability study" (2001). FIU Electronic Theses and Dissertations. 3086.

https://digitalcommons.fiu.edu/etd/3086 
FLORIDA INTERNATIONAL UNIVERSITY

Miami, Florida

TOWARD A UNIFIED THEORY OF TASK-ORIENTED AND RELATIONSHIPORIENTED LEADER BEHAVIOR: A MULTI-COUNTRY GENERALIZABILITY STUDY

\begin{abstract}
A dissertation submitted in partial fulfillment of the requirements for the degree of DOCTOR OF PHILOSOPHY in BUSINESS ADMINISTRATION
\end{abstract} by Ellen Antoinette Drost 
To: Dean Joyce Elam

College of Business Administration

This dissertation, written by Ellen Antoinette Drost, and entitled Toward a Unified Theory of Task-Oriented and Relationship-Oriented Leader Behavior: A Multi-Country Generalizability Study, having been approved in respect to style and intellectual content, is referred to you for judgment.

We have read this dissertation and recommend that it be approved.

Joyce Elam

Mary B. Teagarden

Mary Ann Von Glinow, Major Professor

Date of Defense: August 1, 2000

The dissertation of Ellen Antoinette Drost is approved.

Dean Joyce Elam

College of Business Administration

Interim Dean Samuel S. Shapiro

Division of Graduate Studies

Florida International University, 2001 


\section{DEDICATION}

I dedicate this dissertation to my family and close friends: Big Ellen, Annelies, Henriette, Digna, George, Alex, Caesar, Laura Ellen, Giovanna, Benjamin, Dorothy, Leslie, Nick, Bunnie, Jack, Willem, David, Brice, Teun, Ana, Carmen and Cindy. Without their patience, support, and love, the completion of this work would not have been possible. 


\section{ACKNOWLEDGMENTS}

I wish to thank the members of my committee for their time, valuable comments and patience. Thank you to Dr. Joyce Elam, who agreed to serve on the committee despite her busy schedule. A special thank you to my dear friend and partner in academic travel ventures, Dr. Mary Teagarden, who introduced me to the rigors of academe and who encouraged me to pursue a doctorate degree. A very grateful thank you to my major professor, Dr. Mary Ann Von Glinow, for her support, encouragement, patience, compassion and friendship throughout my studies, and, especially, for having the confidence in me to complete this project. 
TOWARD A UNIFIED THEORY OF TASK-ORIENTED AND RELATIONSHIPORIENTED LEADER BEHAVIOR: A MULTI-COUNTRY

GENERALIZABILITY STUDY

by

Ellen Antoinette Drost

Florida International University, 2001

Miami, Florida

Professor Mary Ann Von Glinow, Major Professor

The theoretical foundation of this study comes from the significant recurrence throughout the leadership literature of two distinct behaviors, task orientation and relationship orientation. Task orientation and relationship orientation are assumed to be generic behaviors, which are universally observed and applied in organizations, even though they may be uniquely enacted in organizations across cultures. The lack of empirical evidence supporting these assumptions provided the impetus to hypothetically develop and empirically confirm the universal application of task orientation and relationship orientation and the generalizability of their measurement in a cross-cultural setting. Task orientation and relationship orientation are operationalized through consideration and initiation of structure, two well-established theoretical leadership constructs. Multiplegroup mean and covariance structures (MACS) analyses are used to simultaneously 
validate the generalizability of the two hypothesized constructs across the 12 cultural groups and to assess whether the similarities and differences discovered are measurement and scaling artifacts or reflect true cross-cultural differences. The data were collected by the author and others as part of a larger international research project. The data are comprised of 2341 managers from 12 countries/regions. The results provide compelling evidence that task orientation and relationship orientation, reliably and validly operationalized through consideration and initiation of structure, are generalizable across the countries/regions sampled. But the results also reveal significant differences in the perception of these behaviors, suggesting that some aspects of task orientation and relationship orientation are strongly affected by cultural influences. These (similarities and) differences reflect directly interpretable, error-free effects among the constructs at the behavioral level. Thus, task orientation and relationship orientation can demonstrate different relations among cultures, yet still be defined equivalently across the 11 cultures studied. The differences found in this study are true differences and may contain information about cultural influences characterizing each cultural context (i.e. group). The nature of such influences should be examined before the results can be meaningfully interpreted. To examine the effects of cultural characteristics on the constructs, additional hypotheses on the constructs' latent parameters can be tested across groups. Constructlevel tests are illustrated in hypothetical examples in light of the study's results. The study contributes significantly to the theoretical understanding of the nature and generalizability of psychological constructs. The theoretical and practical implications of embedding context into a unified theory of task orientated and relationship oriented leader behavior are proposed. Limitations and contributions are also discussed. 
I. INTRODUCTION 1

The Emic-Etic Dilemma in Cross-Cultural Research 2

Construct Comparability in Cross-Cultural Research 3

Research question $\quad 5$

Summary 6

II. A REVIEW OF WESTERN-BASED LEADERSHIP THEORIES AND RESEARCH

Trait Leadership Theories $\quad 7$

Behavioral Theories of Leadership 9

Contingency Theories of Leadership $\quad 12$

Fiedler's Contingency Theory 13

Path-Goal Theory 14

Normative Decision Theory 15

$\begin{array}{lr}\text { Recent Leadership Theories } & 18\end{array}$

$\begin{array}{ll}\text { Transactional Theories } & 18\end{array}$

Implicit Theory 19

Charismatic and Transformational Leadership Theories 21

Conclusion of Leadership Review 24

III A REVIEW OF CROSS-CULTURAL LEADERSHIP THEORIES

AND RESEARCH 26

Behavioral Theories of Leadership $\quad 26$

Contingency Theories of Leadership $\quad 30$

Fiedler's Contingency Theory $\quad 30$

Path-Goal Theory 31

Normative Decision Theory 33

Non-Western Leadership Theories $\quad 35$

Misumi's Performance Maintenance Theory of Leadership 35

Sinha's Nurturant Task-oriented Leadership Model 38

Conclusion of Cross-Cultural Leadership Review $\quad 39$

IV. RESEARCH METHODOLOGY 41

Operationalization of Task Orientation and Relationship Orientation $\quad 42$

Scale and Questionnaire Development $\quad 42$

Reliability and Validity $\quad 44$

Response Bias 45

Independence of Consideration and Initiation of Structure 46

Consideration and Initiation of Structure as

Higher Order Dimensions $\quad 48$ 
Two-Factor Models of Leader Behavior $\quad 50$

Democratic versus Autocratic Styles 51

Task-oriented and Relationship-oriented Leader Behaviors 51

Transactional and Transformational Leadership 53

Conclusions of the Operationalization of Task Orientation and

Relationship Orientation $\quad 53$

Analytical Framework $\quad 55$

Construct Comparability in Cross-Cultural Research $\quad 55$

Means and Covariance Structure (MACS) Analyses 56

Construct Comparability in MACS Analyses Framework $\quad 58$

Assessment of Model Fit $\quad 59$

Test Statistics and Model Fit Indices $\quad 60$

Hypotheses $\quad 62$

General Structural Equation Measurement Model $\quad 62$

Hypothesis 1: Same factor structure $\quad 64$

Hypothesis 2: Equality of factor loadings $\quad 65$

Hypothesis 3: Equality of intercepts $\quad 67$

Hypothesis 4: Equality of measurement error variances $\quad 70$

Hypothesis 5: Equality of latent means 73

$\begin{array}{lll}\text { V. DATA ANALYSIS AND RESULTS } & 75\end{array}$

Data Collection $\quad 75$

Sample $\quad 75$

$\begin{array}{ll}\text { Questionnaire } & 77\end{array}$

$\begin{array}{ll}\text { Preliminary Data Analyses } & 79\end{array}$

Demographic Profile Analysis $\quad 79$

Descriptive Statistics $\quad 82$

Analysis of Variance $\quad 84$

Item Bias Detection Analysis $\quad 86$

Correlations and Reliabilities $\quad 89$

Results of Preliminary Data Analyses $\quad 90$

Hypotheses Tests $\quad 91$

Hypothesis $1 \quad 92$

Hypothesis $2+93$

Hypothesis $3 \quad 93$

Hypothesis $4 \quad 94$

Hypothesis 5

Results 97

Estimated Relative Mean Difference Scores for Task Orientation $\quad 98$

Estimated Relative Mean Difference Scores for

$\begin{array}{ll}\text { Relationship Orientation } & 100\end{array}$

$\begin{array}{lll}\text { VI. DISCUSSION AND CONCLUSION } & 103\end{array}$

Theory and Method 103

Generalizability of Task Orientation and Relationship Orientation 105 
Assessment of Cultural Effects on

Task Orientation and Relationship Orientation

Theoretical Contributions

Methodological Contributions

Limitations

113

Implications for Theory and Practice 


\section{LIST OF TABLES}

TABLE

1. Indicator questions for Consideration and Initiation of Structure

2. Sequence of hypothesis tests for Equality of Latent Means

3. Descriptive statistics for 9 leader behavior items for managers from 12 cultures 121

4. Descriptive statistics for 9 leader behavior items for managers by country/region 122

5. Reliabilities for Consideration and Initiation of Structure by country/region

6. Correlations among 9 Leader Behavior Items for Managers from 12 Cultures

7. Correlations among 9 Leader Behavior Items for Managers from Australia

8. Correlations among 9 Leader Behavior Items for Managers from Canada

9. Correlations among 9 Leader Behavior Items for Managers from China

10. Correlations among 9 Leader Behavior Items for Managers from Gulf Region

11. Correlations among 9 Leader Behavior Items for Managers from Indonesia

12. Correlations among 9 Leader Behavior Items for Managers from Japan

13. Correlations among 9 Leader Behavior Items for Managers from Korea

14. Correlations among 9 Leader Behavior Items for Managers from Mexico

15. Correlations among 9 Leader Behavior Items for Managers from Latin America 135

16. Correlations among 9 Leader Behavior Items for Managers from the Philippines 136

17. Correlations among 9 Leader Behavior Items for Managers from Taiwan

18. Correlations among 9 Leader Behavior Items for Managers from the U.S.

19. Results: Equivalence of Latent Means by Country/Region

20. Estimated Relative Mean Difference Scores for Consideration and 


\section{LIST OF FIGURES}

1. Two-Factor Model for Consideration and Initiation of Structure

2. Hypothesis 1: Same factor structure

3. Hypothesis 2: Same factor loadings

4. Hypothesis 3: Equality of intercepts

5. Hypothesis 3 (IS): Equality of intercepts for Initiation of Structure

6. Hypothesis 3(C): Equality of intercepts for Consideration

7. Hypothesis 4: Equality of Measurement Error Variances

8. Hypothesis 5: Equality of Latent Means, Step 1

9. Hypothesis 5: Equality of Latent Means, Step 2

10. Australia, Equality of Latent Means, Step 1

11. Australia, Equality of Latent Means, Step 2

12. United States, Equality of Latent Means, Step 1

13. United States, Equality of Latent Means, Step 2

14. Canada, Equality of Latent Means, Step 1

15. Canada, Equality of Latent Means, Step 2

16. China, Equality of Latent Means, Step 1

17. China, Equality of Latent Means, Step 2

18. Taiwan, Equality of Latent Means, Step 1

19. Taiwan, Equality of Latent Means, Step 2 
21. Japan, Equality of Latent Means, Step 2

22. Korea, Equality of Latent Means, Step 1

23. Korea, Equality of Latent Means, Step 2

24. Philippines, Equality of Latent Means, Step 1

25. Philippines, Equality of Latent Means, Step 2

26. Gulf Region, Equality of Latent Means, Step 1

27. Gulf Region, Equality of Latent Means, Step 2

28. Latin America, Equality of Latent Means, Step 1

29. Latin America, Equality of Latent Means, Step 2

30. Mexico, Equality of Latent Means, Step 1

31. Mexico, Equality of Latent Means, Step 1 


\section{CHAPTER 1}

\section{INTRODUCTION}

There is a significant recurrence throughout the leadership literature of two distinct leadership behaviors, task orientation and relationship orientation. These two behaviors appear as important phenomena in leadership theorizing and research. They are widely recognized as the most consistently observed and measured behaviors in leadership research (House \& Aditya, 1997). Because organizations are both taskperforming and social institutions, it seems logical that a set of common task-oriented behaviors must be performed to ensure organizational performance, and a set of common relationship-oriented behaviors that must be performed to maintain cohesiveness and social integration among organizational members, units, and processes (Misumi, 1985; House \& Aditya, 1997). As such, these two sets of common behaviors are assumed to be generic behaviors, which are universally observed and applied in organizations across cultures, even though they may be uniquely enacted in organizations across cultures. This study will hypothetically develop and empirically confirm these assumptions.

Because generic behaviors are universally observed and applied behaviors across cultures, the enactment of these behaviors would, inevitably, reflect their cultural context. Such contextual influences often represent pervasive characteristics of a society and are a part of the cultural makeup of the individuals (Little, 2000). Generic leadership behaviors would, inevitability, reflect such influences. Thus, at the behavioral level, 
pervasive cultural characteristics (i.e. influences on behaviors) are reliable and measurable aspects of the individuals brought up in a given culture (Little, 2000). Therefore, it is important to establish generalizabililty of leader behaviors empirically before reliable and valid interpretations of their implicit manifestations can be made.

\section{The Emic-Etic Dilemma in Cross-Cultural Research}

While this study hopes to establish the universal application of two generic leader behaviors, task orientation and relationship orientation, it is also confronted with both the ambiguities of the universal application of theoretical constructs and the generalizability (validity) of equivalent measurements administered across different countries. In crosscultural research, these challenges to theory and method as to whether or not to generalize from one situation or perspective to another is often referred to as the emicetic dilemma (Berry, 1990). The debate between the culture specific or emic approach and the cross-culturally generalizable or etic approach addresses whether behaviors can only be understood (in their full complexity) within the cultural context in which they occur, or whether they are generalizable.

The emic-etic distinction continues to stimulate criticism in comparative methodology and, particularly, in the area of comparability (equivalence). Berry (1990) has attempted to operationalize the emic-etic distinction in a three-step sequence that begins with a study of one's own culture and ends with an explicit act of comparison between two cultures. This three-step approach may lead to a general or derived etic 
theory or measure, in contrast to the current dependence on imposed etic theories and measures primarily drawn from Western societies. Even though Berry's (1990) procedure is preferred, imposed etic theories and measures continue to be more widely used (Drost \& Von Glinow, 1998). The study confronts the emic-etic dilemma and adopts the cross-culturally generalizable approach, or etic approach, by applying U.S. leadership theory, task orientation and relationship orientation, derived from the perceptions of managers across a large number of cultures.

The study's U.S. theory (with task orientation and relationship orientation) leads to a fundamental concern in cross-cultural research, as with most multiple-group comparisons: the identification of theoretical constructs and/or relationships which are valid across different cultures (groups). Conceptually, generic leadership behaviors can be deduced from general statements based on empirical data that evaluate the perceptions of leader behaviors across cultures. However, instances in which such generalizations are manifested in dissimilar behaviors in different cultures may also occur. It could be that certain cultures give unique meanings to task orientation and relationship orientation. Thus, in cross-cultural research, comparability (equivalence) is often treated from a measurement perspective (van de Vijver \& Tanzer, 1997).

Construct Comparability in the Measurement of Cross-Cultural Research

The extant leadership literature, which will be reviewed in Chapters 2 and 3, has publicized broad generalizations about the differences in leadership traits and behaviors 
of cultural groups, which, upon closer examination, are based on psychometrically poor measures and inadequate assessment procedures. In order to avoid making such broad generalizations, equivalence of measures (also referred to as measurement equivalence), should be demonstrated instead of simply assumed (Poortinga \& Malpass, 1986; van de Vijver \& Tanzer, 1997; Little, 2000). Thus, if the universal application of task orientation and relationship orientation, which are investigated here, is to be understood, measurement equivalence of these leader behaviors must be established (to ensure crosscultural comparability) before valid interpretations of cross-cultural differences can be made.

Because measurement equivalence is a prerequisite for valid comparisons across cultural groups (van de Vijver \& Tanzer, 1997), it is important that researchers use an analytical framework that can ensure construct comparability. Mean and covariance structures (MACS) analyses framework is a powerful technique in that it extends standard structural equation modeling (SEM) techniques wherein mean-level information of the indicators is structured (analyzed) along with the variance-covariance information typical of SEM (Little, 1997). The MACS analyses framework is particularly useful in cross cultural research, because it simultaneously validates (test) hypothesized factor structures across (cultural) groups and can test the nature of (systematic) cultural influences on many aspects of the constructs (i.e. means, variances, covariances, correlations) (Little, 1997). 
This study seeks to establish the universal application of two generic leadership behaviors, task orientation and relationship orientation. In order to establish such an application, the study must focus on measurement equivalence of these two leadership behaviors in a multi-cultural setting and must address whether the empirical similarities and differences discovered are measurement and scaling artifacts or reflect real crosscultural differences.

Task orientation and relationship orientation are operationalized through consideration and initiation of structure, two well-established theoretical leadership constructs, consideration and initiation of structure. The study uses a powerful analytical framework, mean and covariance structures analyses (MACS), to assess their measurement equivalence and to identify real cross-cultural differences (if they exist) across 10 national and 2 regional settings.

The research question of the study is:

Are task orientation and relationship orientation generalizable across cultures? 
Summary

The study seeks to establish the universal application of task orientation and relationship orientation. Mean and covariance structures (MACS) analyses are used to establish generalizability of task orientation and relationship orientation in a multicultural setting and to address whether the empirical similarities and differences discovered are measurement and scaling artifacts or reflect "true" cultural effects.

In Chapter 2, a review of post-WWII U.S. leadership theories reveals how taskorientation and relationship-orientation have come to be widely accepted as universal leadership behaviors. In Chapter 3, a further review of the leadership literature in crosscultural settings reveals that while task-orientation and relationship orientation are identifiable, there are no consistent patterns in terms of frequency, extent, and crosscultural comparisons. Chapter 4 discusses the research methodology and presents a framework for testing measurement equivalence and for identifying (real) cross-cultural differences. Analytical and statistical procedures, along with the study's findings, are presented in Chapter 5. The study concludes in Chapter 6 with a discussion of the results, limitations, and implications for future research. 


\section{CHAPTER 2}

\section{A REVIEW OF WESTERN-BASED LEADERSHIP THEORIES AND RESEARCH}

It would be a daunting task to organize and integrate all that is known about leadership and cross-cultural leadership. Many excellent reviews and texts have been written with that purpose in mind (e.g. Bass, 1990; House \& Aditya, 1997; Yukl, 1998). This literature review reveals that within the major leadership theories, task orientation and relationship orientation have, in varying degrees, always emerged. It addresses the three major perspectives in leadership research: the trait, behavioral and contingency perspectives. The cross-cultural literature review further testifies to the presence, albeit not clearly understood, of task orientation and relationship orientation.

\section{Trait Leadership Theories}

Trait leadership theories emphasize the personal attributes of leaders and ascribe leader success to extraordinary abilities such as persuasive powers, unusual foresight, penetrating intuition, and steadfast energy (Yukl, 1989). During the 1930's and 1940's, hundreds of studies were conducted to reveal these "traits". However, this immense research effort failed to find any traits that would guarantee leadership success (Yukl, 1989). The main problem with early trait research was that there was little empirically substantiated personality theory to guide the search for leadership traits (House \& Aditya, 1997). Moreover, test-measurement theory was not well developed during early trait 
research. Little information about the psychometric properties of the trait measures were reported; thus, many of the measures had limited validity. Consequently, the lack of theory and valid measurement instruments both contributed to the near consensus among leadership scholars that trait research was pointless.

In the early 1970 s, substantial advancement in measurement theory and clarification of theoretical issues led to several new empirically supported traits which led to the re-emergence of the leadership trait paradigm (House \& Aditya, 1997). One of the major criticisms of Trait Theory was that traits must be stable and predictable over time and across situations (Davis-Blake \& Pfeffer, 1988). Schneider (1983) observed, however, that traits are predictive of an individual's behavior in select situations, rather than across situations. Similarly, House, Shane and Herold (1996) noted that individual dispositions are possibly stable over extended periods of time, but not inevitably over a lifetime. In a reevaluation of earlier research by Stogdill (1948), House and Baetz (1979) found that intelligence, pro-social assertiveness, self confidence, energy-activity, and task-relevant knowledge were consistently supported as recurring traits. In their metaanalysis of 35 earlier studies of six leader traits, Lord, De Vader, and Alliger (1986) found that three traits--masculinity, dominance and intelligence--were all significantly related to follower perceptions of leadership (House \& Aditya, 1997).

Even though theoretical clarifications of leader traits were given in the Schneider (1983) and House, Shane and Herold (1996) studies, the findings of the meta analysis 
provide no explanation for the associations between the traits and leader effectiveness (House \& Aditya, 1997).

In sum, traits that consistently emerge from trait theory and research to date are physical energy, intelligence greater than the average intelligence of followers led, prosocial influence domination (House \& Baetz, 1979), achievement motivation, self confidence, flexibility and the motives of the leader motive profile (House \& Aditya, 1997). Furthermore, the effects of leader behaviors and leader effectiveness are improved by the relevance of the traits to the situation in which the leader acts. Moreover, traits have a stronger influence on leader behaviors when the situation permits the expression of individual disposition. Thus, the behavioral manifestation of a trait is stronger in weak situations and weaker in strong situations (House \& Aditya, 1997).

Behavioral Theories of Leadership

Following World War II, researchers disappointed with the "trait" approach to leadership turned their attention toward leader behaviors (what managers do on the job). Researchers hoped that an emphasis on the observable characteristics of leadership might prove productive both in terms of describing/explaining the specific nature of leadership activity and in identifying patterns of behaviors that are related to effective leadership (Chemers, 1997, p. 22). The studies at The Ohio State University (i.e. Fleishman, 1953; Fleishman, Harris, \& Burt,1955), the University of Michigan (e.g. Bowers and 
Seashore,1966; Likert,1961, 1967) and Harvard University (Bales \& Slater, 1955) are good examples of the behavioral approach.

The most comprehensive program of research on leadership behavior was initiated at The Ohio State University. The goal of this program was to develop an instrument that would accurately measure leader behavior. This eventually resulted in the Leader Behavior Description Questionnaire (LBDQ), one of the most longstanding and widely used instruments in leadership research (Chemers, 1997). Factor analyses of the questionnaire (Halpin \& Winer, 1957) revealed that subordinates perceived leader behaviors to fall into two factors or behavioral clusters. The first of these, consideration, included relationship-oriented behaviors as showing concern for subordinates and being supportive and friendly. The second factor, initiation of structure, referred to behaviors designed to organize and structure group activities, define relationships, assign subordinates toward task accomplishment (Chemers, 1997).

The LBDQ factors were related to superior and subordinate evaluations of leaders and to a variety of dependent measures including follower satisfaction, group harmony , team performance, turnover rates, and more. Although many individual studies were able to relate consideration or initiation of structure to particular outcome measures, the relationships between the two leader behavior factors and outcome measures shifted, being positively related in one study to negatively related or unrelated in another (for an extensive discussion, see Bass, 1990, Chapter 24). 
Around the same time, researchers at the University of Michigan (Kahn, 1953;

Katz \& Kahn, 1951) were studying leader behavior from a different perspective. Using interviews with subordinates of industrial supervisors, the Michigan group identified two general leader behaviors: production-oriented and employee-oriented behaviors (Chemers, 1997). Production-oriented supervisors emphasized planning, direction and productivity, while employee-oriented supervisors were characterized as having good rapport with subordinates, an open and accepting management style, and a concern for the problems and feelings of subordinates (Chemers, 1997).

In a series of studies conducted by Bales and his colleagues (Bales \& Slater, 1955) at Harvard University, a method was developed for recording group behavior. Their interaction process analysis, which logged the behavior of college students in leaderless group discussion, revealed two distinct types of emergent leaders. The most active individuals were engaged in organizing, summarizing, and directive behaviors and were called task-specialists, while the second type, the socio-emotional specialists, acted to reduce interpersonal tensions, raise morale, and instigate group participation (Chemers, 1997, p. 22).

These three research programs, using different methodologies, reached consensus by identifying two leader behavior patterns. One pattern focused on the task, by organizing and directing the work of others (e.g. initiation of structure, task-oriented leader behavior) while the other focused on maintaining a positive emotional 
interpersonal atmosphere among the group members (e.g. consideration, relationshiporiented leader behavior) (Chemers, 1997).

It is important to stress, however, that ratings of behavior reflect the perceptions of the rater, not actual behavior, and that perceptions are influenced by many factors in addition to actual behavior. Just as personal traits may be somewhat important depending on the situation, leaders also must tailor their behavior to the situation. Simply emphasizing universal approaches of traits, behaviors or styles is unlikely to be sufficient to explain the dynamics of the leadership process (Chemers, 1997). The behavioral approach also failed to examine critical situational factors. Effective leaders must adapt their actions to the requirements of the task and characteristics of the subordinates who perform the task (Yukl \& Van Fleet, 1992; Dorfman, 1996). Thus, a more complex and integrative approach to understanding leadership was needed, which involved the interaction of a leader behaviors, such as consideration and initiation of structure, with important aspects of the (leadership) situation. This is considered next in the contingency theories of leadership.

\section{Contingency Theories of Leadership}

In the early $1960 \mathrm{~s}$, as trait and behavioral approaches in earlier studies fell out of favor, the contingency approach turned leadership research in a dramatic new direction. The contingency approach was the first to emphasize the importance of situational factors such as the nature of the work performed by the leader's unit, the leader's discretion and 
authority, the subordinate ability and motivation, the nature of the external environment and the role requirements dictated by the manager's superiors, peers, subordinates, and outsiders, and how they interact with leader personality and behavior (Yukl, 1989; Chemers, 1997).

There are three well-known approaches to contingency theory: Fiedler's Contingency Theory, Path-Goal Theory and Normative Decision Theory. The following is a brief discussion of their approaches and directions.

Fiedler's Contingency Theory

Fiedler's ground breaking Contingency Theory (Fiedler, 1967; Fiedler \& Chemers,1984) is probably the most researched model of leadership (Bass, 1990). Fiedler posited a two-way interaction between a measure of leader task-motivation versus relationship motivation, and a measure of what was initially referred to as situational control (House \& Aditya, 1996). The personality-motivational orientation of the leader, measured by the least preferred coworker (LPC) score, shows the extent to which the leader's primary motivation is task-oriented or relationship-oriented (Dorfman, 1996). In a personal communication with Robert House (House \& Aditya, 1997) in May of 1997, Fiedler stated that his contingency hypothesis was based on three zones of situational control. He hypothesized that task-motivated leaders perform best in situations of high and low control while relationship-motivated leaders perform best in situations of moderate control. 
Despite its breakthrough, contingency theory was criticized for inconsistent empirical evidence (Schriesheim \& Kerr, 1977; Ashour, 1973; Strube \& Garcia, 1981; Vecchio, 1983; Peters, et al., 1985). So far, supporters of the theory argue that numerous validity studies using sophisticated meta-analyses techniques have sufficiently supported the major premises of the theory (Dorfman, 1996; House \& Aditya, 1997).

Path-Goal Theory

The Path-Goal Theory (Evans, 1970; House, 1971, House \& Dessler, 1974, Schriesheim \& Von Glinow, 1977) was also intended to reconcile prior inconsistent findings concerning task-oriented and relationship-oriented leader behaviors. The theory specified a number of situational moderators of relationships between task-oriented and relationship-oriented leader behaviors and their effects (House \& Aditya,1997). The theory is follower-oriented given that the attributes of followers, such as their competence and personality needs, affect the appropriate leader behavior. Task and environment, such as task structure and complexity, are also considered. The revised theory (House \& Mitchell, 1974) uses four specific leader behaviors: directive leadership, supportive leadership, achievement-oriented leadership, and participative leadership.

Recent reviews of the Path-Goal Theory suggest that it has not been adequately tested (Evans, 1996; Schriesheim \& Neider, 1996). House and Aditya (1997) consider this to be attributed to the complexity of the model, which specifies four leader behaviors, 
several situational and follower trait moderators, five intervening variables (follower expectancies and valences), and two dependent variables (follower satisfaction and performance).

Inasmuch as the original Path-Goal Theory was a theory of dyadic relationships between supervisors and subordinates, House's 1996 version of the Path-Goal Theory is a theory of relationships between superiors and work unit effectiveness (House \& Aditya, 1997). The boundary conditions are more clearly defined for hypothesis testing than the older Path Goal Theory. Eight classes of leader behavior and situational contingencies are specified that moderate the effect of these behaviors on work unit performance. However, House's 1996 version remains to be tested.

Normative Decision Theory

Vroom and Yetton (1973) developed a model of participation, the Normative Decision Model, which was reformulated by Vroom and Jago (1988), also referred to as the Vroom-Yetton-Jago model (Chemers, 1997). The model implies that leaders are more effective if they use decisions that are appropriate in a particular situation (Yukl, 1989). Vroom and his colleagues argue that one of a leader's privileges is controlling the process by which decisions are made. Their model is both prescriptive (e.g. normative) in specifying the boundaries that determine which type of decision processes should be used and descriptive in collecting data on the kinds of processes that leaders actually do employ. The model is operationalized as a prescriptive model in the form of 
a decision tree (House \& Aditya, 1997). It includes a variety of decision strategies to be considered against a set of situational constraints that are guided by a number of rules designed to protect the ultimate decision from various deficiencies (Chemers, 1997). The decision strategies fall into three general categories; autocratic, consultative, and democratic (group). The model suggests that all situations which require decisions on the part of a group/leader has an ideal decision strategy and the effectiveness of any decision will result accordingly.

The validity of the model is tested by measuring whether the leader/manager acted in accordance with the prescriptions of the model An important limitation of prescriptive models concerns the extent to which leaders are able to vary their behaviors to match certain situations. The Vroom-Yetton-Jago model and the Path-Goal Theory assume that leaders can easily change their behavior to match the situation. In contrast, Fiedler and Chemers $(1974,1984)$ present leadership style as a personality trait that is stable over time and not easily changeable. The empirical evidence is controversial. Vroom reported that only $10 \%$ of the variance in managers' responses to his standard problems was attributed to the differences between managers, and the variance attributed to the differences between the problems was 3 to 5 times as great (Chemers, 1997, p. 52). Bass and his associates (Bass \& Valenzi, 1974) analyzed a large data set relating decision styles to intrapersonal, interpersonal, and organizational variables. Their findings indicated that managers had a tendency to use one style or set of related styles more than others. That is, they showed individual differences reflecting stable patterns of autocratic or participative styles. Thompson and Chemers (1993) reported results of two studies in 
which managers were asked to report the likelihood that they would change decision styles or behaviors in various situations. Half the managers were told that the purpose of the research was to examine common decision making behavior of managers, while the other half was told that the study was about differences between female and male managers (Chemers, 1997, p. 53). The ratings given to several autocratic and democratic decision strategies or task-oriented and relationship-oriented leader behaviors varied considerably from one condition to another. Responses indicated that managers were more self-conscious when they thought that gender issues were being studied.

The three contingency theories reviewed here focus on the extent to which the leader emphasizes task-oriented, directive, and structuring issues versus relationshiporiented, supportive, and participative behaviors. There is overlap across the three contingency theories on the specification of critical situational factors. All three models include variables that relate to the clarity and certainty of the task requirements and goal paths (Chemers, 1997). The theories attempt to make explicit predictions relating person and situation variables. However, Fiedler's Contingency Theory differs to some extent from the other two theories in that the leader's behavior is a product of stable, ingrained cognitive and emotional reaction patterns. Both the Path-Goal Theory and Normative Decision Theory assume that the leader is capable of using any behaviors required by the situation (Chemers, 1997).

Even though some of the major predictions of Fiedler's Contingency Theory and Path-Goal Theory were supported by meta-analyses (Peters, Hartke \& Pohlman, 1985; 
Strube \& Garcia, 1981; Wofford \& Liska, 1993), the theories did not do well overall, most likely due to their complexity and, therefore, limited capability of being empirically tested and replicated. With the waning of scholarly interest in the contingency theories, several theories were recently introduced which intended to explain different aspects of the leadership phenomena. These theories were very much concerned with the nature of leader-follower relationships, with the cognitive processes underlying evaluations and perceptions of leadership and with the characteristics of charismatic/transformational leaders. These more recent leadership theories are discussed next.

Recent Leadership Theories

Transactional Theories

The transactional theories or exchange theories of leadership are focused on how leaders can motivate followers by creating fair exchanges and by clarifying mutual responsibilities and benefits (Chemers, 1997). The leader-follower relationships are seen as reciprocal exchanges in which leaders and followers create a transaction that allows for mutual satisfaction of goals and needs.

George Graen and his associates have extensively studied transactional/exchange approaches, known as Grain's Vertical Dyadic Linkage Model, but more commonly referred to as Leader-Member Exchange (LMX) Theory (Graen \& Cashman, 1975; Dansereau, Graen, \& Haga, 1975; Graen, 1976; Graen \& Scandura, 1987). LMX Theory 
examines relationships, as opposed to behavior or traits of either followers or leaders.

The theory views leader-follower relationships in terms of social exchanges in which both leaders and followers perform effectively in response to high-quality relationships with each other (House \& Aditya, 1997). LMX theory postulates that high-quality relationships between leaders and followers are built on mutual respect and trust (such as mentor-protégé relationships) (Chemers, 1997). It is not clear, however, whether these are universal attributes of high-quality relationships.

Despite the substantial volume of literature on dyadic-relationships, the questions raised by the theory are still being answered. For instance, little attention has been dedicated to specific leader behaviors that foster high-quality relationships. The theory implies that the superiors need to be supportive, engage in open communication, and delegate a substantial amount of discretion to the subordinate in conducting their work. The leader behaviors implied in these theories are those behaviors observed by earlier researchers as relationship-oriented behaviors and general (or universal), rather than specific (or individual), supervision (e.g. Katz \& Kahn, 1953). However, there is little evidence relevant to this proposition (House \& Aditya, 1997).

\section{Implicit Leadership Theory}

Over the many years of leadership research, developments in the field of psychology had a valuable effect on leadership theorizing. Personality, cognitive and social psychology contributed to the understanding of the effects of cognitive structures 
on interpersonal perception. Similar issues in leadership concepts led Robert Lord and his associates to advance the Implicit Theory of Leadership (Lord et al., 1984) which posits that leadership perceptions form a number of hierarchically organized cognitive categories, each of which is represented by a prototype. Implicit theories are cognitive frameworks or categorization systems that are in use during information processing to encode, process, and recall specific events and behavior (Bass, 1990, p. 376). Thus, as Lord and Mayer (1991) argued, a person is a leader in part by matching the particular traits and behaviors of an observed individual to the prototype for a leader in a specific context.

Evidence for the existence of leader prototypes has been produced in laboratory studies (e.g. Lord \& Mayer, 1991; Gioia \& Sims, 1985; Rush, Thomas \& Lord, 1977). Implicit theories of leadership can be manipulated in advance to get raters to rate the same observed leadership behavior, such as task-oriented leader behavior, differently. If we believe and are told that a leader is effective in carrying out his or her job, it will influence what the type of leadership we perceive to exist in the later performance observed in that leader (Bass, 1990, p. 376). For example, in a laboratory study by Gioia and Sims (1985), subjects viewed the videotapes of contingently reinforcing leadership behavior by managers in action. When the managers were presented to the subjects as effective leaders before the subjects saw the tapes, the subjects described the managers as being significantly higher in task-oriented behavior (initiation of structure) than the managers who were presented to the subjects as ineffective before the same tapes were viewed (Bass, 1990, p. 376). This perspective raises the possibility that there are some 
universally endorsed leader attributes and behaviors that comprise implicit leadership theories.

To gain an understanding of the kind of leadership in which followers are persuaded to go beyond their own self-interests and become truly dedicated to the leader's mission, we must address charismatic and transformational leadership.

Charismatic and Transformational Leadership Theories

House's original Path-Goal Theory led to the development of the 1976 Theory of Charismatic Leadership (House, 1977), which laid the groundwork for the charismatic and transformational leadership (Bryman, 1992) paradigm. These leadership theories include the Theory of Transformational Leadership, built on the transactionaltransformational conceptualization derived from Burns (1978), and later developed and operationalized by Bass (1985), the Attributional Theory of Charismatic Leadership (Conger \& Kanungo, 1987), the visionary theories by Kouzes and Posner (1987), Bennis and Nanus (1985) and Tichy and Devanna (1986), and the Value Based Theory of Leadership (House et al., 1996). These theories all address the effects that transformational leaders have on followers. For example, House and Shamir (1993) theorized that charismatic leaders are able to transform the needs, values, preferences, and aspirations of followers from self-interest to collective interest. Charismatic leaders are able to accomplish these effects by engaging followers' self-concepts and linking valued aspects of those self-concepts to the leader's vision and mission, and by arousing 
unconscious motives relevant to task accomplishment. In other words, the bases for motivational subordinate effort and commitment are made intrinsic (i.e. tied to the follower's self-concept) rather than extrinsic as they are in transactional leadership, based on a quid pro quo of effort for personal gain (Chemers, 1997, p. 89).

In the mid 1980s, Bernard Bass and his colleagues developed an influential measurement model, the Full Range of Leadership Model, which places transformational and charismatic leadership, among others, on an active-passive leadership continuum and describes how these leadership types are related (e.g. Bass, 1985; Bass \& Avolio, 1989; Yammarino \& Bass, 1990; Bass \& Avolio, 1993; and others). Their efforts ultimately resulted in the development of a survey instrument, the multifactor leadership questionnaire (MLQ) (Bass \& Avolio, 1989). A recent meta-analysis by Lowe, Kroeck and Sivasubramaniam (1996), based on 32 correlations between the charisma scale of the MLQ (Bass \& Avolio, 1989) and independent ratings of leader effectiveness, indicated a mean corrected correlation of 0.35 . A second meta-analysis by these authors, based on fifteen correlations between charisma and subordinates' ratings of their superiors' effectiveness indicated a corrected correlation of 0.81 (House \& Aditya, 1997).

In a recent literature review, House and Aditya (1997) concluded that the charismatic theories offer inadequate or untested explanations of the processes by which the charismatic leader behaviors affect followers. The authors note that Transformational Leadership Theory (Bass, 1985) rests on Maslow's theory of motivation, which has been largely disproved by empirical tests (Wahba \& Bridwell, 1975), while, the charisma 
theory advanced by Conger and Kanungo (1987) rests on an attributional explanation of the effects of charismatic leaders. However, the specific attribution processes to which the theory alludes are not clearly specified. These processes, therefore, have yet to be empirically demonstrated.

It is clear that transformational and charismatic leadership theories provide important insights, but that serious conceptual weaknesses need to be addressed to make the theories more plausible (Yukl, 1999). The theories do not explain the underlying influence processes clearly, nor do they specify how the leader behaviors are related to these processes. Yukl (1999) provides an excellent evaluation of conceptual weaknesses in transformational and charismatic leadership theories. Of particular interest here is Yukl's criticism of Bass using the label "full range leadership theory" for his transformational theory (Bass, 1985) when the theory neglects to include a measure of task-oriented behavior relevant for effective leadership.

Moreover, the charismatic and transformational theories create the impression that charismatic leadership is equally applicable to all organizational situations. As one critic remarked: "One of the most surprising features of the New Leadership is that it heralds a return to the 'one best way' approach to thinking about leadership that was characteristic of most trait and style research" (Bryman, 1992, p. 157, in Shamir \& Howell, p. 257). Similarly, in a recent Special Issue of Leadership Quarterly on charismatic leadership, Shamir and Howell (1999) noted that most studies on charismatic and transformational leadership pay little attention to the role of context and situational 
factors. Beyer (1999), in the same Special Issue, questioned how different research in transformational leadership is when compared to earlier research from the behavioral paradigm. In a reply, Bass (1999) concurred that his measures of transformational leadership captured variance not accounted for by the established constructs of consideration and initiation of structure. However, as Beyer (1999) noted, that capturing additional variance does not prove that these new approaches to leadership are different from the older theories, but could be a methodological artifact.

\section{Conclusion of Leadership Review}

Despite the lack of universal acceptance of the aforementioned theories of leadership reviewed here, they have contributed to the development of a cumulative body of informed and, to some extent, empirically supported leadership research. Early trait research was shown to have identified traits that have some claim to universality, at least in the U.S. (Lord et al, 1986). The behavioral perspective identifies two distinct patterns of leader behavior, task orientation and relationship orientation that continue to have importance in explaining leader effectiveness (House \& Aditya, 1997). The contingency theories extend the behavioral theories by adding situational moderators to explain leader effectiveness. The theories concur that there is a single best way for a leader to act within a given situation. Despite their groundbreaking approach to leadership theorizing, the contingency models are complex and ambiguous and, as a result, provide only partial tests of the theories. The transactional or dyadic theories imply leader behaviors that are similar to the task-oriented and relationship-oriented behaviors observed by earlier 
researchers. Transformational and charismatic leadership theories are conceptually weak and return to a "one best way" (Bryman, 1992, p. 157, in Shamir \& Howell, 1999, p. 257) approach to thinking about leadership that can be seen in earlier leadership trait and style approaches discussed earlier.

Noteworthy is the recurrence throughout the literature of two distinct leadership behaviors, task-orientation and relationship-orientation. These two behaviors appear throughout the literature review as important phenomena in leadership theorizing and research. Therefore, it is likely that these two leadership behaviors are generic to the application of leadership and universally accepted and effective across organizations, industries, and cultures (House \& Aditya, 1997). It is this hypothesis, heretofore undeveloped theoretically and undemonstrated empirically, which this study tests. The role of task orientation and relationship orientation in other cultures is the topic of the next chapter. 


\section{A REVIEW OF CROSS-CULTURAL LEADERSHIP THEORIES AND RESEARCH}

The U.S.-based theories of leadership just reviewed point to task orientation and relationship orientation as the dominant generic leadership behaviors to emerge in 50 years of leadership research. We can view them as general U.S. leader behavior approaches or styles. This review of cross-cultural leadership literature has produced conflicting but nevertheless compelling evidence of the universality of these two leader behaviors.

This chapter addresses three major theories in cross-cultures in leadership research: behavioral leadership theories, contingency theories and non-western theories of leadership and related measurement and methodological inconsistencies.

Behavioral Leadership Theories

Many studies have supported the importance of the task-orientated and relationship-orientated leadership dimensions across cultures. Cross-cultural studies by Bond and Hwang (1986), Sinha (1980) and Ayman and Chemers (1983) have supported task-oriented and relationship-oriented leader behaviors to increase subordinates' satisfaction. Tscheulin (1973), in his review of task-oriented and relationship-oriented leadership studies, confirmed that the similarity of results across 20 years of research 
with different cultures and different analytical methods were significant (Dorfman, 1996).

Studies in Israel by Fleishman and Simmons (1970) and Rim (1965) extended U.S.

findings to Israeli foremen, head nurses and industrial supervisors. They found that the most influential people were those high in both consideration and initiation of structure (Dorfman, 1996).

However, results of other studies in different cultures are not so consistent. For instance, Anderson (1983) found that considerate leader behavior was detrimental to midlevel managerial effectiveness in New Zealand (Bass, 1990). In a comparison of Turkish and American first-line supervisors, Kenis (1977) observed that American supervisors were perceived to be more considerate and participative than Turkish supervisors, but equal in structuring behaviors. However, whereas consideration, participation and structure were related to satisfaction with supervision for Americans, only consideration was related to satisfaction with supervision for the Turkish sample (Dofrman, 1996, p. 287). The Bass, et al. (1979) 12-nation data for Exercise Supervise concluded that the French and Latin Americans regarded being considerate as unimportant at all levels of management (Bass, 1990). Moreover, consideration was stressed by fast-track managers but not by slow-climbing managers in Portugal, Italy, and Spain. However, consideration was de-emphasized by managers with accelerated careers in Latin America, India, France, Belgium and Scandinavia.

In general, cross-cultural studies support the importance of considerate leader behavior in increasing subordinates' satisfaction (Dorfman, 1996). This should not be 
surprising since supportive leaders show concern for subordinates and are considerate and available to listen to subordinates' problems (Howell et al., 1994). Contrary findings are infrequent, but they do occur, as, for example, in Anderson's (1983) study in New Zealand where considerate behavior was found to be detrimental to midlevel manager effectiveness. Moreover, Bennett (1977) found that Chinese and Filipino bank managers were less relationship-oriented than their Western counterparts and concluded that effectiveness of this behavior may be dependent on cultural norms.

In their reviews of cross-cultural leadership, Tannenbaum (1980), Bhagat, et al. (1990), Bass (1990), and Smith and Peterson (1988) draw different conclusions concerning task-oriented and relationship-oriented leader behaviors. For instance, while Smith and Peterson (1988) note that the two leadership functions have not been consistently supported in individualistic societies (where the theories were developed), they conclude that studies conducted in collectivistic societies have given more consistent support to the two leadership behaviors. Completely contrasting results were reached by Bhagat, et al. (1990, 1989), these researchers concluded that (the well known dimensions of) consideration and initiation of structure are not as appropriate in non-Western cultures as they are in the U.S.

Moreover, some evidence exists that cultures differ in their perception of considerate behaviors. Studies indicated that cultures varied in their perceptions of managerial supportive behaviors and often differed in the factor structure of leadership scales measuring Consideration and Initiation of Structure (Anderson, 1983; Ayman \& 
Chemers, 1982; Drost \& Von Glinow, 1998). Ayman and Chemers (1982) came up with a single factor (instead of the two factors) for consideration and initiation of structure for European managers in comparison to U.S. managers as perceived by their respective subordinates (Bass, 1990). Drost and Von Glinow's (1998) study of Mexican managers and employees indicated different factor structures for managers and employees as perceived by their subordinates. It is not clear from the studies, however, whether the differences are measurement artifacts or real cross-cultural differences. In summary, results from the cross-cultural studies reviewed here provide evidence that culture matters, but it is difficult to specify the precise nature of cultural contingencies.

This raises several methodological issues that are relevant to the literature just cited. First, any scale (survey items) that is developed without concern for the possible emic (Berry, 1990) (i.e., insider familiar with the culture) nature of the construct (behavior measured) in question (without any culturally contingent measures) will provide ambiguous findings and interpretations and fail to generalize the other culturally different settings. Second, the constructs themselves are multifaceted and the definitions vary among the originators of the theories. Moreover, within a particular theory, the conceptualization and operationalization of the constructs themselves and the measurement instruments have changed over time. As Podsakoff and Schriesheim (1985) found in their review of LBDQ studies, many researchers failed to mention which version of the LBDQ they used or how they had modified the questionnaire (Bass, 1990). These variabilities create numerous measurement problems for the researcher (Dorfman, 1996). 
In short, the ambiguity of interpretation, especially in cross-cultural studies, is problematic.

The contingency approach sought to explain some of the ambiguities of crosscultural research that consists of identifying specific dimensions of cultural variation and using these dimensions to understand how leadership changes from culture to culture. Triandis (1993) proposed to use culture as a moderator in a contingency model. The contingency approaches to cross-cultural leadership research are discussed next.

Contingency Theories of Leadership

Fiedler's Contingency Theory.

Initial tests in Holland and Belgium were supportive of Fiedler's Contingency Model (Fiedler, Meuwese \& Oonk, 1961) (Least Preferred Coworker (LPC) model). Bennett (1977) conducted a study of managers in the Philippines and Hong Kong that provided some evidence that culture might play a moderating role in the contingency model (Dorfman, 1996). Bennett (1977) found that high-performing Chinese managers were more relationship-oriented (high LPC scores), whereas Filipino managers were more task-oriented (low LPC scores).

Although Bennett (1977) acknowledged that the study was not a thorough test of the LPC model, he concluded from the results that a person-oriented or relationship- 
oriented dimension to leadership, which is similar to consideration, may depend on cultural norms for its effectiveness. On the contrary, other studies of considerate leadership determined the opposite-it is the initiation of structure leadership dimension, which is similar to task-orientation, that is culturally contingent (Dorfman, 1996). Test of Fiedler's contingency model in Japan have been ambiguous. LPC scores seem to depend on the particular sample, and serious questions about the reliability and construct validity of the LPC measure have arisen in Japan.

\section{Path-Goal Theory}

In Path-Goal Theory, the subordinate's task-related need for structure is hypothesized to moderate subordinate's reactions to the leader's directive or considerate behavior (Chemers, 1996).

Dorfman and Howell (1988) used the Path-Goal Theory to investigate the moderating impact of culture differently. They looked at the influence of culture by comparing individuals who showed a strong association with particular cultural values (i.e. power distance, collectivism/ individualism, paternalism, and masculinity). They reported that supportive leader behaviors were not moderated by cultural beliefs. Full and partial moderating effects of culture were found for directive leader behavior. Directive leadership had a maximal effect on employee performance and attitude when the employee held strong cultural beliefs in the cultural dimensions that were expected to 
promote directive leader behavior (e.g. high power distance between supervisor and subordinate is believed appropriate).

Al-Gattan (1985) found support for the model in Saudi Arabia, when he investigated the relationships between the four leader behaviors (achievement-oriented, participative, directive, and supportive) and subordinate performance and satisfaction moderated by the subordinate's task, need strength, and locus of control. All four leader behaviors related significantly to satisfaction with supervision and most of the hypotheses were fully or partially supported by the model.

Howell, et al. (1994) incorporated Yukl's multiple linkage model, a meta-theory that includes a wider range of leader behaviors, into the original Path-Goal model to test the generalizability of six leader behaviors across five countries in North America and Asia. They found complete universality for supportive behavior and contingent reward, partial universality for directive and charismatic behaviors, and cultural specificity for participative and contingent punishment behaviors. In comparing the impacts of leader behaviors in Western versus Asian cultures, Howell and his colleagues were surprised to find that the U.S. was the only culture where leader participation had a significant, positive effect on subordinates attitudes. The combination of high individualism (Hofstede,1980) and high participative management in the U.S. most likely contributed to this culturally-unique outcome in the U.S. sample (Dorfman,1996). Dorfman et al. (1997) replicated the findings in a similar study of leader behaviors across five countries (U.S., S. Korea, Japan, Taiwan, and Mexico) which provided evidence for both 
conceptual and measurement equivalence and to some extent for generalizability of supportive, contingent reward and charismatic leader behaviors.

Normative Decision Theory

One of the best supported situational or contingency leadership theories is the Vroom and Yetton (1973) normative decision model and their revised model (Vroom \& Jago 1988), whereby managers select an appropriate level of participation for problem solving among pre-specified problem sets. However, there is little research investigating the validity of the normative decision model in cross-cultural situations (Dorfman, 1996).

Preliminary cross-cultural investigations confirmed that the model might be helpful in understanding participatory leadership in other cultures. Bottger, Hallein, and Yetton (1985) investigated the potential use of participation among 150 managers from Africa, Papua-New Guinea, and the Pacific Islands using the standard Vroom and Yetton model. They found that in situations of low power and low structure, managers' participation was highest, compared to situations of high power and high structure, a clear indication that when managers feel powerless and problems are not structured, a participatory style is preferred. The authors attributed these findings to the level of managerial education across countries, rather than to cultural influences. However, no attempt was made to rule out alternative cultural hypotheses (Dorfman, 1996). 
Considerable cultural differences have been found in participative leadership that can be conceptualized along a continuum that ranges from highly autocratic decisions to complete delegation (Dorfman, 1996). The differences along this continuum become especially apparent in cross-cultural research, when data from more freely socialized societies are compared to data from more rigid societies where core beliefs of the local culture are accepted (Bass, 1990).

Particularly noteworthy are predictions of participation based on Hofstede's $(1980,1991)$ power distance scores. For instance, in a five-country study of leader behaviors, Dorfman and colleagues (1997) found no support for participative leadership in Mexico. On the contrary, Pelled and Hill (1997) observed that participative management enhanced performance and lowered turnover in a study of maquiladora plants in Northern Mexico, even though Mexico's rigid culture seems incongruent with participative management practices as evidenced by the Dorfman et al. study (1997). Similarly, Vargas and Johnson (1993) noted that managers responded positively when asked whether or not their plants used participative practices and also reported that employee involvement was beneficial to the plant's performance.

Jago et al. (1993) observed some contrasts between U.S. managers and additional European counterparts. For instance, Swiss, Austrian and German managers were the most participative, Czech and Polish managers the most autocratic, and the French and U.S. managers somewhere in between. Interestingly, while Polish managers were more likely to be participative on trivial matters, both U.S. and Polish managers were prone to 
become more autocratic as subordinate conflict increased. However, in general, Jago et al.'s study supported the prediction that participation scores are higher for low power distance cultures (Dorfman, 1996).

Many studies support the notion that general supervision is favored by workers high in power distance and authoritarian countries, such as those in Latin America, the Middle East, South East Asia (Whyte, 1963; Singh \& Arya 1965; Meade \& Whittaker 1967; Meade 1967; Redding \& Casey 1975; Kenis, 1977; Hofstede, 1980; Bass, 1989,1990). However, conflicting evidence exists in the studies mentioned earlier (i.e. Pelled \& Hill, 1997; Vargas \& Johnson, 1997). At this time there is no compelling evidence to either support or refute the universality of participative leadership found in Western societies (Dorfman, 1996).

Non-Western Leadership Theories

Misumi's Performance-Maintenance Theory of Leadership

An extensive study of non-Western leader behavior was conducted by Misumi (1985) in Japan. Misumi (1985) believed that leaders exhibited two basic leadership functions in all situations: performance functions $(\mathrm{P})$, which are related to task requirements; and maintenance functions $(\mathrm{M})$, which are related to relationships among workers. Misumi's (1985) performance (P) and maintenance $(M)$ functions resemble the consideration and initiation of structure constructs of the Leader Behavior Description 
Questionnaire (LBDQ) (Stogdill, 1963) and the task-oriented and relationship-oriented leader behaviors.

While many theories of leadership in the U.S. regard the task-oriented and relationship-oriented leadership functions as independent constructs, Musimi's (1985) research indicated consistently that the best Japanese leaders combined task-oriented behavior with considerate, relationship-oriented behavior (Misumi \& Peterson, 1985; Chemers, 1997). Comparable effects were reported for Iranian managers (Ayman \& Chemers, 1983) and for Indian managers (Sinha, 1993).

The similarity of the main constructs of Misumi's (1985) performancemaintenance theory to those of U.S. theories raises the question about the universality of basic leadership constructs (e.g. consideration and initiation of structure) and the generalizablity of their measurement. Smith et al. (1989) carried out a cross-cultural comparison of Misumi's (1985) performance and maintenance constructs in the U.S., Great Britain, Hong Kong and Japan. They administered surveys to shop floor workers and their immediate supervisors, which contained 20 items from the performancemaintenance measure (Misumi \& Peterson, 1985) and an additional 36 items developed to describe specific behaviors in which supervisors handled concrete problems.

Factor analyses of the scales yielded the two-factor solution, with a task/performance factor and a consideration/maintenance factor in all four countries. Even though the pattern of factor loadings on the performance/maintenance factors was 
similar in all countries, for certain items the factor loadings (the regression weights) were not always similar across countries. For instance, items such as "Does your superior treat you fairly?' loaded on the maintenance factor in each country, and "does your superior urge you to complete work within a specified time?" loaded on the performance factor in all countries. But, items such as "Does your superior let you know about plans and tasks for your day-to-day work?" indicated much stronger factor loadings (regression weights) on the performance factor in the Anglo cultures than in the Asian countries (Chemers, 1997, p. 129-130). These differences in factor loadings could be attributed to measurement artifacts or real cross-cultural differences. These methodological issues will be discussed in details in the research methodology chapter.

The specific behaviors from the performance-maintenance survey indicated a more dramatic influence of cultural differences. Of the 36 specific behaviors surveyed, only 8 loaded on the same factors in all cultures. For example, in the Asian sample, a supervisor who discusses a subordinate's poor performance with other members of the group rather than confronting the subordinate directly indicated strong maintenance behavior, whereas the same behavior was perceived as negative maintenance behavior in the Western sample. Another example relates to the high maintenance supervisor in China, the supervisor resolved personal difficulties (tactfully) in an indirect manner, whereas the high maintenance supervisor in Britain and the U.S. shared the task-related information with the subordinate (Chemers, 1997, p. 130). 
Misumi's (1985) PM theory of leadership is unique in that it uses items to reflect leadership behaviors that are broadly applicable to many contexts (e.g. country, type of organization, group) while it also tailors items to the specific context (research setting). Furthermore, Misumi observed that the meaning of a particular leader behavior might change in the context of other leader behaviors, a type of leadership interaction (Dorfman, 1996, p. 304). In contrast to the behavioral approach to leadership theories developed in the U.S., but consistent with contingency theories of leadership, Misumi (1985) posits that the two leader behaviors are contingent upon context, thus contributing to the consistency and universality of leader behavior.

Sinha's Nurturant Task-Oriented Leadership Model

Similar to Misumi's (1985) research in Japan, Sinha (1984) developed a Nurturant Task-oriented model (NT) that incorporated a combination of leadership styles in India (Dorfman, 1996). According to the theory, nurturant task-oriented leaders are considerate, care for their subordinates, and are committed to their growth. However, their nurturance is dependent on the subordinate's task accomplishment-the leader becomes a caring source given that the subordinate works hard, respects and obeys the supervisor and is highly productive (Dorfman, 1996). Evidence suggests that the effectiveness of NT is contingent upon a number of variables such as the subordinate's need for a dependency relationship and acknowledgment of a hierarchical relationship (Sinha, 1984; Dorfman, 1996). The NT model closely resembles other contingency 
theories such as Path-Goal theory and Misumi's PM (1985) theory, thus lending additional support for a universality argument.

The similarity of the main constructs of Misumi's (1985) PM theory and Sinha's (1984) NT theory to those of many U.S. theories of task-oriented and relationshiporiented leader behaviors begins to reinforce the universality of these basic leadership functions and the generalizability of their measurement (Chemers, 1997). However, it is also apparent that cultural variability in values, beliefs and needs influences the ways in which these two functions are most effectively executed.

\section{Conclusions on Cross-Cultural Leadership Review}

Despite insufficient empirical support for the generalizability of leadership theories across cultures, there are some indications that a number of leadership behaviors are indeed universal. Similar to the U.S.-based leadership review, task orientation and relationship orientation have been the most frequently measured and observed leader behaviors in cross-cultural research. While the logic suggesting universality of these two leader behaviors is compelling, some of the conflicting results from the literature review suggest that it is difficult to conceptualize these two leader behaviors across cultures. The ambiguities found in the literature make it difficult to specify the precise nature of cultural contingencies and leave the researcher with some important questions. 
For example, in three cross-cultural studies on task-oriented and relationshiporiented leader behaviors by Smith et al. (1989) in the U.S., Great Britain, Hong Kong and Japan; by Ayman and Chemers (1982) in the U.S. and Europe, and by Drost and Von Glinow (1998) in Mexico, conclusions are based on different factor structures than those hypothesized by the theories. This raises a fundamental question in cross-cultural research as to whether task orientation and relationship orientation are generalizable across cultures. The extant literature suggests that task orientation and relationship orientation are universally observed and applied leader behavior, but under which conditions or contexts these behaviors prevail is not clear. It is also unclear whether or not the observed similarities and differences are measurement artifacts or "true" (valid) cross-cultural differences in the perceptions behaviors.

This study addresses these challenges and supports the cross-cultural generalizing or etic (Berry, 1990) approach by using U.S. leadership theory to establish the universal application of task-oriented and relationship-oriented leader behaviors in a multi-cultural setting. The next chapter will present the research methodology. 


\section{CHAPTER 4}

\section{RESEARCH METHODOLOGY}

The last chapter concluded that if cross-cultural leadership research is to progress, comparability of theoretical constructs must be established before valid interpretations of cross-cultural differences can be made. This chapter examines measurement and theoretical issues relevant to the analytical framework proposed in this chapter.

The chapter has three sections. The first section discusses the operationalization of task orientation and relationship orientation through two well-established theoretical constructs, consideration and initiation of structure and examines the constructs' scale and questionnaire development, reliability and validity, response bias, independence of the factors, higher order factor dimensions. The section concludes that two-factor models of task-oriented and relationship-oriented leader behaviors prevail and that these models can be successfully operationalized through consideration and initiation of structure.

The second section focuses on measurement and theoretical issues, discusses construct comparability or measurement equivalence (these terms are used interchangeably from here on) in multiple-group comparisons and introduces mean and covariance structures (MACS) analyses and the MACS analytical framework. The third section presents the hypotheses. 
Operationalization of Task Orientation and Relationship Orientation

The LBDQ (Halpin \& Winer, 1957; Stogdill, 1963), discussed on page 6, was developed from 150 statements describing different aspects of leader behavior. From these results, the Ohio State University researchers discovered two factors that indicated that subordinates perceived leader behavior to consistently fall into two independent categories. The first category concerned relationship-oriented leader behaviors, which they labeled consideration. The second category concerned task-oriented behaviors, which they labeled initiation of structure (see page 6 for discussion).

Scale and Questionnaire Development

Three leader behavior description questionnaires were developed over the years. The LBDQ consisted of 40 statements, which measured the two factors, consideration and initiation of structure (Hempdill \& Coons, 1957). An industrial version, the Supervisory Behavior Description Questionnaire (SBDQ) came next, followed by the LBDQ-Form XII, which consisted of 20 statements which measured the two constructs and expanded from the initial two-factors to a broader array of leader-behavior dimensions (Bass, 1990).

The LBDQ and the SBDQ differed with regard to their measures of initiation of structure (Bass, 1990). The LBDQ contained a subset of 15 items which asked 
subordinates to describe the actual structuring behavior of their leader, such as establishing well-defined patters of communication and detailing ways to get the job done (Halpin, 1957). The SBDQ consisted of 20 items that also asked subordinates to describe the actual structuring behavior of their leader, however, initiation of structure was intended to reflect the extent to which the leader organized and defined interactions among group members, established ways to get the job done, scheduled work, criticized subordinates, etc. (Schriesheim, House \& Kerr, 1976). The SBDQ initiation of structure measure included a wider spectrum of structuring behaviors based on group interaction than the LBDQ, while such items on the LBDQ addressed communication and ways to get the job done.

Based on a theoretical analysis of the differentiating roles among group members, Stogdill (1963) proposed 10 additional patterns of behavior involved in leadership. Conceptually these behaviors were independent of consideration and initiation of structure and were to be included in the LBDQ, hence the LBDQ-XII. These additional behaviors included (1) representation — behaves as the representative of the group, (2) reconciliation - resolves conflicting organizational demands and eases confusion in the system, (3) tolerance of uncertainty - is able to accept uncertainty without distress or anxiety, (4) persuasiveness - uses persuasion and dispute effectively, shows confidence, (5) tolerance for freedom-allows followers opportunity for initiative, decision, and action, (6) role retention-actively implements the leadership role, rather than conceding leadership to others, (7) production emphasis-compels productive output, (8) predictive accuracy - demonstrates foresight and the know-how to foresee outcomes accurately (9) 
integration-maintains a tight organization and settles inter-member conflicts, (10) influence with supervisors-maintains friendly relations with superiors, has power with them, is determined to move up in the organization (Bass, 1990, p. 516).

Noteworthy is the inclusion of a persuasiveness factor (factor 4), which anticipated the more recent focus on the measurement of transformational and charismatic leader behaviors (Bass, 1990).

Reliability and Validity

Even though all three questionnaires/versions have been used extensively throughout the literature, an assessment of their reliability was necessary because the content of the scales varied causing difficulties in establishing their validity. Moreover, researchers deleted items, modified the wording of items for use in a particular study, or failed to mention which version they used, which complicated matters even further. Over the years, however, many reviews and meta-analytical studies reported stable and consistent measures of consideration and initiation of structure from one situation to another (Korman, 1966; Schriesheim \& Kerr, 1974; Hunt, Osborn, \& Schriesheim, 1978; Podsakoff \& Schriesheim, 1985; Fisher \& Edwards, 1988). Reported reliability measures for consideration and initiation of structure were .93 and .81 for the LBDQ, .81 and .68 for the SBDQ, and .90 and .78 for the LBDQ-XII (Schriesheim \& Kerr, 1974; Bass, 1990). 
In general, factor validation studies of the LBDQ-XII scales suggested that each factor was strongly dominated by a single appropriate scale. In their four studies using 9 of the LBDQ-XII scales, Stogdill, Goode and Day $(1963,1964,1965)$ reported that just two factors, representation and role retention, emerged with only their representative scale items. However, when all items (12 scales) of the LBDQ-XII were correlated and factor analyzed, somewhat different factors structures emerged. One general factor emerged with numerous and highly loaded items of persuasiveness. Items from the other scales that loaded on the general factor included being persuasive, being able to resolve demands, enjoying the leadership role, influencing superiors, and structuring the task to be performed. This general factor suggested that leaders were seen as being supportive of their followers' welfare and considerate, as well. The remaining factors tended to be composed of items from single scales. Moreover, consideration separated into two factors, discussed later in connection with Miller's (1973) hierarchical analysis. Overall, these findings imply that the behavior of leaders is complex in structure and that followers are able to differentiate among different aspects of leader behavior (Bass, 1990).

Response Bias of Consideration and Initiation of Structure

Despite empirical support for the validity of consideration and initiation of structure, the scales suffered from halo effects and were burdened by other response errors, such as leniency and social desirability (Schriesheim, Kinicki \& Schriesheim, 
1979). Therefore, it was difficult to establish whether they were true valid measures of consideration and initiation of structure (Bass, 1990).

Schriesheim, Kinicki and Schriesheim (1979) reviewed the effects of leniency in five studies and concluded that leniency response bias, the tendency to describe others in favorable but probably untrue terms, did not particularly affect descriptions of initiation of structure. However, although consideration and leniency are conceptually distinct, they concluded that consideration reproduced an underlying leniency factor when applied in field settings. Moreover, the consideration items were not socially unbiased and predisposed to leniency. As a result, leniency explained much of the variance in consideration. As Fleishman (1973) noted, this might explain why consideration tends to correlate higher with other evaluative variables as compared to initiation of structure.

Independence of Consideration and Initiation of Structure

Given their original orthogonal factor structure, consideration and initiation of structure are, however, not independent factors (Bass, 1990). In a review of studies using the LBDQ, Schriesheim, House, and Kerr (1976) reported a positive median correlation of 0.45 between the two factors. Similarly, in a subsequent review of 10 studies using the LBDQ-XII, the median correlation between consideration and initiation of structure was 0.52. Higher correlations were observed when job pressure (as a situational variable) was strong. In a review of 22 industrial and 9 military studies, Weissenberg and Kavanagh (1972) concluded that a significant positive correlation was found between 
consideration and initiation of structure, even though managers think they should behave as if consideration and initiating structure are independent. Katerberg and Hom (1981) reported positive between-group and within-group correlations between the two leader behaviors.

In his review of leadership studies, Bass (1990) reported significant negative correlations between consideration and initiation of structure in studies that used the SBDQ, which included some autocratic items (Bass, 1990). The median correlation was, however, only -0.05 . In a comprehensive review of 32 studies using the SBDQ, Fleishman (1989) reported a similar correlation of -0.02 between consideration and initiation of structure. Although all three versions contained some degree of arbitrary punitive performance, the SBDQ, in particular, was the most marked and contained items that measured punitive, subjective, coercive, and dominating behaviors that affected the scores for initiation of structure. The reliability of initiation of structure on the SBDQ was raised from 0.68 to 0.78 when the 3 punitive items (e.g. "the leader demands more than we can do", "needles subordinates for greater effort") were removed from its scoring scale (as in Bass, 1990, p. 513). The LBDQ-XII was considered most free of such autocratic items (Schriesheim \& Kerr, 1974).

Even though factorially and conceptually independent, the intercorrelations between consideration and initiation of structure suggested that the leader's tendencies to be considerate and to initiate structure are typically found to correlate moderately with each other. Because researchers were not satisfied that the behavior of leaders could be 
adequately described with just two factors, alternative and additional scales were developed to provide a more detailed profile of behavior.

Consideration and Initiation of Structure as Higher-Order Dimensions

The introduction of the LBDQ-XII (Stogdill, 1963), which measured additional domains of leader behavior, stimulated an interest in fine-tuning the basic content of consideration and initiation of structure and related measures. For instance, Yukl (1971) established the viability of a three-factor approach including consideration, initiation of structure, and centralization of decisions. Wofford (1971) expanded the framework of leader behavior to five dimensions including personal enhancement, personal interaction, group achievement and order, dynamic achievement, security, and maintenance (Bass, 1990, p. 519).

In a study of 170 Canadian school principals described by 1551 teachers, Brown (1967) obtained scores on each of the 12 factors of the LBDQ-XII. Brown reported that two higher-order factors accounted for 76 percent of the total variance explained. Production, structure and representation clustered around an axis of initiation of structure, while tolerance of uncertainty, tolerance of freedom, and consideration clustered about an axis of consideration (Bass, 1990). The remaining loadings patterns fell between the clusters at the extremes of these two orthogonal axes. Similar results were obtained for the LBDQ-XII by Stogdill et al.'s (1965) study of university presidents. Production 
emphasis, structure, representation and persuasiveness clustered around the first axis and uncertainty, freedom and consideration clustered around the second axis (Bass, 1990).

The lack of equivalence between the LBDQ's measures of consideration and initiation of structure and similar instruments reported in these studies gave Miller (1973) the impetus to collect 160 items from 9 standard instruments often used in leadership research to obtain a better understanding of the similarities and differences in the measures of consideration and initiation of structure. Miller extracted 73 nonduplicative items from the following questionnaires: the LBDQ (Halpin \& Winer, 1957), Survey of Organizations (Taylor \& Bowers, 1972), interaction process analysis (Bales, 1950), the Job Descriptive Index (Smith, Kendall, \& Hulin, 1969), the Orientation Inventory (Bass, 1962), the Continuum of Leadership Behaviors scale anchors (Tannenbaum \& Schmidt, 1958), six statements describing decision making styles (Vroom \& Yetton, 1973), the five bases of social power (French \& Raven, 1959), and measures of the least preferred co-worker (LPC) (Fiedler, 1967).

Miller (1973) collected data from 200 respondents in 10 organizations from different industries. Miller factor analyzed the data by stipulating a hierarchical solution starting with a two-factor solution, then repeating the analysis stipulating a three-factor solution, and so on. Each solution reflected well-known leader behavior factors. Interestingly, the initial two-factor solution clearly paralleled consideration and initiation of structure (Bass, 1990). Subsequent higher-order factor analysis, based on an oblique 
rotation, again yielded the two higher order factors of consideration and initiation of structure.

The validation studies reviewed here indicate that a two-factor solution is repeatedly extracted in the analysis of leader-behavior descriptions. Even though a multifactor solution appears warranted due to the complexity of leader behavior, task orientation and relationship orientation continue to be the most widely recognized and observed behaviors in leadership research, implying the universality a the two-factor model of leader behavior.

Two-Factor Models of Leader Behavior

From the literature reviewed, it appears that two-factor models of leader behavior have dominated the theories of leadership research. Examples are democratic versus autocratic leadership, participative versus directive leadership and task-oriented versus relationship-oriented leadership. Each pair includes behaviors such as orientation toward employees, providing support and maintaining group cohesiveness (consideration) versus the facilitation of work, goal achievement, and production orientation (initiation of structure) (Bass, 1990). The relationships between the two-factor models of leader behavior and consideration and initiation of structure are discussed next. 
The various scales of consideration and initiation of structure each contain a variety of autocratic and democratic elements (Bass, 1990). Yukl and Hunt (1976) investigated the relationship between Bower and Seashore's (1966) four leadership styles of emphasis on goals, facilitation of work, support and facilitation of interaction and the LBDQ. Their study indicated that support correlated 0.66 with consideration and 0.61 with initiation of structure. Alternatively, emphasis on goals correlated 0.64 with consideration and 0.76 with initiation of structure, while facilitation of work correlated 0.56 with consideration and 0.64 with the initiation of structure. Taken somewhat differently, these findings suggest that a general factor may permeate all the scales.

Task-oriented and Relationship-oriented Leader Behaviors

Consideration emphasizes the leader's orientation to followers (e.g. " sees that subordinates are rewarded for a job well done", stresses the important of people and their satisfaction at work," in Bass, 1990, p. 525), as well as participative decision making behavior ("gets approval of subordinates important matters before going ahead", puts subordinate's suggestions into operation," in Bass, p. 525). Initiation of structure emphasizes the concern with the task ("emphasizes the meeting of deadlines," sees that subordinates work to their full capacity," in Bass, p. 525), as well as directiveness ("decides in detail what should be done and how it should be done," makes attitudes clear," in Bass, 1990, p. 525). Besides, hypothetically opposite to exhibiting 
consideration is behavior that is, unsupportive, uncaring and exploitative (Bernardin, 1976) while the lack of initiation of structure implies conditions to continue without giving directions or being task oriented.

Fiedler's (LPC) Contingency model of leadership claims to measure task-oriented and relationship-oriented leader behaviors, although controversy continues about the effectiveness of Fiedler's LPC model. The LPC (least-preferred coworker) scale describes the one person with whom the respondent could work least well. This description is made by marking 16 items that have opposite poles (e.g. pleasantunpleasant; cold-warm; friendly-unfriendly; distant-close; and so on). A high LPC score was considered to indicate a relationship-oriented person, whereas a low LPC score was considered to indicate a task-oriented person (Bass, 1990). Meuwese and Fiedler (1965) reported that high and low LPC leaders did not differ significantly in the total scores for consideration and initiation of structure. In a study by Graham (1968), high LPC leaders were described as being higher in consideration and structure than were lowLPC leaders. Similarly, Yukl (1968) noted that low-LPC leaders (task-oriented) tended to be described as high in initiation of structure and low in consideration. Yukl (1971) and Kavanagh (1975) reasoned that task-oriented behavior is implicit in initiating structure, but that subordinates are still able to influence their superior's decisions. 
In a study of 294 MBA students, Seltzer and Bass (1987) reported that the scales of initiation of structure on the LBDQ-XII correlated $0.53,0.55$, and 0.59 with charisma, individualized consideration, and intellectual stimulation, respectively, on the MLQ (Bass \& Avolio, 1989). Consideration on the LBDQ, on the other hand, correlated $0.78,0.78$, and 0.65 with the same MLQ transformation measures, respectively, and 0.64 and -0.23 , with the same MLQ transactional leadership measures, respectively. Moreover, strong associations exist between transformational leadership and consideration. In a study of 264 retail chain-store employees describing their supervisors, Peterson, Phillips, and Duran (1989) reported that the MLQ scale of charismatic leader behavior correlated higher with measures of consideration ( 0.48 with maintenance orientation and 0.74 with support) than measures of initiation of structure ( 0.16 with pressure for production and 0.22 with assigning work) (Bass, (1990). Clearly, these associations among the various leadership concepts and consideration and initiation of structure indicate the two-factor dominance of task-oriented and relationship-oriented leader behaviors.

Conclusion of the Operationalization of Task Orientation and Relationship Orientation

The validation studies reviewed here indicate that a two-factor solution is repeatedly extracted in the analysis of leader-behavior descriptions. Although conceptualized and factored as two independent constructs, consideration and initiation of structure, were generally found to correlate moderately with each other, as were 
autocratic versus democratic and task-oriented and relationship-oriented leadership.

Thus, leaders who are high on one factor are often high on the other factor, as well (Bass, 1990, p. 524).

The psychometric review indicated that when 3 punitive items from the initiation of structure scale were eliminated from the SBDQ, its reliability raised substantially. Moreover, the usual negative associations between initiation of structure (as measured by the SBDQ), and other behavioral variables (e.g. job satisfaction, morale) became positive when the punitive/coercive elements were removed (Bass, 1990). Moreover, the autocratic items on earlier versions of the LBDQ may have a negative effect, especially on job satisfaction. Leniency effects are likely to continue to bias the results along with halo effects when single sources of variance are used to evaluate both leader behavior and outcomes (Bass, 1990).

Leadership concepts such as orientation toward employees, human relations skills, providing for satisfaction of needs, group-maintenance and providing support are all analogous to consideration and initiation of structure as was indicated in numerous correlational and factor analytic studies. A similar conclusion can be drawn for initiation of structure that parallels concepts such as the utilization of technical skills, enabling the achievement of goals, differentiation of the supervisory role, a production orientation and the facilitation of work. (Miner, 1973; Bass, 1990). In sum, evidence abound that task orientation and relationship orientation are generic leader behaviors that can be reliably and validly operationalized (measured) through consideration and initiation of structure. 


\section{Construct Comparability in Cross-cultural Research}

A fundamental concern in cross-cultural research is ensuring construct comparability or measurement equivalence of psychological constructs (for simplicity, construct comparability and measurement equivalence will be used interchangeably throughout the paper) when testing for differences across groups. Cross-cultural studies typically hypothesize cultural differences in individual perceptions and attitudes on the basis of scale scores (Cheung \& Rensvold, 2000), however, observed differences may be due to measurement artifacts unrelated to the constructs of interests (Cheung \& Rensvold, 2000; Little, 1997; Mullen, 1995; van de Vijver \& Leung, 1997; Berry, Poortinga, Segall, \& Dasen, 1992). Thus, a question often raised is whether test or scale scores obtained in different cultural populations can be interpreted in the same way across these populations (van de Vijver \& Tanzer, 1997).

Because observed variables reflect both common and specific sources of variance, cultural effects may influence both sources at the construct level (Mulaik, 1972). Construct comparability will hold if cultural effects have influenced only the commonvariance components of the construct's indicators and not their unique-variance components (see Little, 1997, 2000). If cultural effects influence the unique variance components of the construct's indicators (e.g. an item is perceived differently or is poorly translated in one group), nonequivalence would result (Little, 1997). Measurement 
nonequivalence, even though an important analytical outcome in cross-cultural research, does not allow for quantitative construct comparisons (Little, 1997).

The advantages of measurement equivalence are that the constructs are generalizable to each cultural context, sources of error and bias are minimal, cultural differences have not inconsistently affected the constructs basic measurement characteristics, and the reliable and true properties of the constructs can be assessed for possible group differences (Little, 1997). In sum, measurement equivalence of psychological constructs must be ensured before results can be meaningfully compared across cultures and specific hypotheses about the underlying constructs can be tested.

Mean and Covariance Structures (MACS) Analyses

Mean and covariance structures (MACS) analyses are recommended (e.g. Cheung \& Rensvold, 2000; Little, 1997, 2000) to ensure measurement equivalence when testing for mean differences of (latent) constructs in a multiple group settings. The MACS analyses framework used here is adopted from Cheung \& Rensvold (2000), Mullen (1995) and, in particular, from Little (1997). The discussion follows their premises.

MACS analyses are an extension of standard structural equation modeling techniques (e.g. confirmatory factor analysis), in that mean-level information about the indicators is also analyzed along with the usual variance-covariance information of SEM analyses (Little, 2000). MACS analyses are particularly useful for multiple group 
comparisons of (latent) constructs, because they simultaneously validate (test) the hypothesized factor structures in each group and detect possible differences on the reliable and true properties of the constructs (Little, 1997; 2000).

In SEM, measurement equivalence is tested in hierarchically linked levels (Bollen, 1989; Cheung \& Rensvold, 1999; Little, 1997, 2000, Mullen, 1995). The least restrictive level, structural equivalence or equality of factor structures, suggests that each group associates the same indicators with the same underlying constructs (factors) (Cheung and Rensvold, 1999). The next level, factorial invariance, indicates that each group ascribes approximately the same weight to manifest variables (indicators) indicated by equal factor loading parameters (Cheung \& Rensvold, 1999). This implies that respondents give more or less the same meaning to the items. A higher level, intercept invariance, or strong factorial invariance(e.g. Meredith, 1993; Little, 1997) implies that neither one of the groups has a tendency to systematically respond higher or lower than the other group (i.e. acquiescence response set bias), which affects the validity of the scale. The next highest level, equality of measurement error variances (Mullen, 1995) or strict factorial invariance (Meredith, 1993; Little, 1997), implies that each group has equivalent measurement error variances, addressing the reliability and validity of scales across groups (Mullen, 1995; van de Vijver \& Tanzer, 1997). Both strong factorial invariance (Meredith, 1993; Little, 1997; Cheung \& Rensvold, 2000) and strict factorial equivalence (Meredith, 1993; Mullen, 1995) indicate measurement equivalence of (latent) construct. When measurement equivalence is established, the latent aspects of the constructs (i.e. means, covariances) can be tested for possible cultural influences on 
the constructs across groups. The equality of latent variables (constructs) is a precondition for comparing correlations of latent variables across groups (Little, 1997; Cheung \& Rensvold, 2000).

The remainder of the chapter presents the MACS analytical framework, provides an overview of the theory of SEM, discusses the "fit" of structural equation models to the (sampled) data and justifies the hypotheses.

Construct Comparability in (MACS) Analyses Framework

The MACS analyses framework addresses two distinct questions about construct: comparability: (1) whether the constructs' operational definitions are equivalent in two or more groups, and (2) whether the latent elements of the constructs (e.g. the constructs' means or covariances) are similar in two or more groups. This second question focuses on hypotheses about possible "true" cultural influences (i.e. differences in the constructs' means and/or covariances) on the constructs. In a MACS analyses framework, the reliable components of the measurement space, e.g. indicator loadings, intercepts, error variances are tested for measurement equivalence before the components of the latent space (e.g. means and covariances) are addressed.

Construct comparability (either at the measurement level or the latent level) occurs when the parameters of a construct's manifest variables are not significantly different across groups (Little, 1997). This hypothesis is tested as follows: (1) cross- 
group equality constraints are placed on each corresponding measurement parameter, (2) latent variances and means are allowed to vary freely in groups that follow, and (3) a simultaneous model estimation (mathematical) procedure estimates whether the model fits the data, by estimating an implied covariance matrix which is then compared to the observed covariance matrix (i.e., actual data) (Jorekog \& Sorbom, 1989; Little, 1997; Kelloway, 1998). Measurement equivalence is reasonable if the equality constraints across groups generate the best possible fit values that hold across groups and do not have a significant influence on model fit (i.e. the model fits the data) (Little, 2000).

\section{Assessment of Model Fit}

The assessment of model fit (the fit between the actual data and the estimated, hypothesized model) in MACS analyses is evaluated using either a statistical or a modeling rationale (see Little, 2000). When a statistical rationale is used, equivalence across groups is tested as a nested-model comparison between a model in which specific parameters across groups are constrained to equality and one in which the parameters in all groups are freely estimated (Bollen, 1989). The difference in $\chi^{2}$ (Chi-square difference test) between the two models is a test of the equality restrictions. If $\chi^{2}$ is nonsignificant, the statistical test indicates no cross-group differences in the estimated parameters. When a modeling rationale is used, practical fit indices determine the overall adequacy of a model. Generally, this later rationale is used for models with many constrained parameters because the $\chi^{2}$ is an extremely sensitive fit index/statistic, especially when estimated on large sample sizes (Joreskog \& Sorbom, 1989). From this 
perspective, if a model with many constrained parameters indicates adequate practical fit, then the set of constraints are considered acceptable approximations of the data. The assessment of model fit is discussed in the next section.

Test Statistics and Model Fit Indices

The $\chi^{2}$ statistic may be viewed as a test statistic for the hypothesis that discrepancies between the model and data are due only to sampling variation rather than model departures from underlying assumptions (Medsker, et al., 1994). The $\chi^{2}$ statistic is distributed asymptotically as a $\chi^{2}$ distribution when statistical conditions are met. The degrees of freedom of the model serve as a standard by which to judge whether $\chi^{2}$ is too large. Large $\chi^{2}$ values correspond to bad fit between the model and data and small values to good fit (Joreskog \& Sorbom, 1989). However, the $\chi^{2}$ statistic is an overly sensitive index of model fit, especially for evaluating models with numerous constraints on large sample sizes. Consequently, if a model with many constraints indicates adequate practical fit, then the set of constraints can be regarded as reasonable approximations of the data (Little, 2000).

A precise criterion for using model fit as test statistics has not been established (Little, 1997). One of the earliest and most frequently reported fit indices that compares a model's fit against other nested models is the Tucker-Lewis Index, TLI, (Tucker \& Lewis, 1973). Tucker and Lewis (1973) implied that if a difference in Rho between a 
freely estimated and constrained model is less than approximately .05 then the information gained by adding an additional dimensions is fairly trivial relative to the freely estimated model. Similarly, McGraw and Joreskog (1971) agreed that a difference of .022 between a freely estimated and constrained model was negligible and opted for invariance on the basis of parsimony and minimal difference in fit (Little, 1997). Following Little (1997), a modeling rational may be justified if: (1) overall model fit is acceptable (using an acceptable standard such as Rho .90); (2) the difference in fit between the freely estimated and constrained model is negligible (e.g. $\Delta \mathrm{TLI}<.05$ ), and (3) the accepted model is substantively more meaningful and parsimonious than the alternative model.

Wheaton (1983) and Medsker et al. (1994) suggest that researchers report multiple fit indices to represent different aspect of the model. The Comparative Fit Index (CFI) (Bentler, 1990), is known as the best approximation of the population parameter for an overall model fit and is less sensitive to sample size (Medsker, et al., 1994). The CFI ranges between 0 and 1 , with values exceeding 0.90 indicating a good fit to the data (Kelloway, 1998). The CFI will be reported to evaluate overall model fit. The TLI is an incremental fit index and has proven to be a less bias estimator of the asymptotic value (Mulaik et al., 1989). Again, TLI ranges between 0 and 1, with values exceeding 0.90 indicating a good fit to the data (Mulaik et al., 1989). The TLI will be reported to test nested models. The Root Mean Squared Error of Approximation (RMSEA), developed by Steiger (1990), is based on the analysis of residuals, with smaller values indicating a better fit to the data (Kelloway, 1998). Steiger (1990) suggests that values below 0.05 
indicate very good fit to the data and values below 0.10 a good fit to the data. The advantage of using the RMSEA is that it also provides a test of the significance of the RMSEA by testing whether the value obtained is significantly different from 0.05 (suggested by Steiger as a very good fit to the data). Pclose tests the null hypothesis that the population RMSEA is not greater than 0.05 .

Hypotheses

\section{General Structural Equation Measurement Model}

A comprehensive introduction to structural equations modeling and its application is given in Bollen (1989). A basic overview will be provided here to explain the measurement models in the subsequent hypotheses.

In SEM, two or more groups are compared on the latent variable (construct), not on a linear combination of the manifest (indicator) variables. Following Bollen (1989) the structural equation measurement model for $x_{i}$ can be written as

$$
x=\Lambda_{\mathrm{x}} \xi+s+e
$$

where vector $x$ represents the input variables (indicators), matrix $\Lambda$ (lambda) the coefficients of regression (factor loadings) of the input variables $x$ on their corresponding 
theoretical latent construct, $\xi$ (xi), and $s$, a vector of systematic variance components, unrelated to $\xi$, and $e$, a vector of random errors of measurement.

The general measurement model for latent variables, which is a general confirmatory factor analysis model, can be defined by the parameters

$$
\Lambda_{x}^{(g)}, \Theta_{\delta}^{(g)}, \Phi^{(g)}
$$

where the superscript $(g)$ refers to the gth cultural sample, $g=1,2, \ldots, \ldots G$.

The $\Lambda_{x}$ (lambda) matrices contain parameters that are the (structural) coefficients that link the latent constructs and the indicator variables. The $\Theta_{\delta}$ (theta-delta) matrices are covariance matrices of the errors of measurement. The $\Phi$ (phi) matrices are covariance matrices of the latent constructs.

Figure 1 illustrates the specifications of the two-factor model for consideration and initiation of structure. Consideration is measured with 5 indicators and initiation of structure is measured with 4 indicators. The model hypothesizes that the relation between the latent constructs, consideration and initiation of structure, and each indicator is the same across 12 cultures. Table 1 shows the indicator questions for both latent constructs. 
The general hypothesis for measurement equivalence is that the measurement models are equivalent (the same relation of indicators to latent variable holds) between two or more groups. Table 2 presents the proposed hierarchy of hypotheses tests proposed summary form (Little, 1997, 2000; Cheung \& Rensvold, 2000; Mullen, 1995; Bollen, 1989).

Hypothesis 1: Same Factor Structure

The hierarchy begins with the most general hypothesis and tests the fit of a theoretically derived baseline model. The pattern of significant factor loadings between manifest variables (indicators) and latent variables (constructs) is tested for equivalence (invariance) across groups, that is

$$
\mathrm{H}_{\text {form }}: \Lambda_{\text {form }}^{(1)}=\Lambda_{\text {form }}^{(2)}=\ldots=\Lambda_{\mathrm{x}}^{(\mathrm{G})}
$$

for all groups $(g)$. Factor loadings are not constrained to be equal across groups when testing for factorial structure. In order to identify the model, the measurement scale of each latent construct may be fixed arbitrarily by setting one of its factor loadings $(\lambda s)$ equal to one (1) for each factor across groups. The other factor loadings ( $\lambda \mathrm{s})$, along with the diagonal elements of the error variances matrix, $\Theta$, and the covariance matrix, $\Phi$, are not constraint (also referred to as "free") across groups. The measurement model is depicted in Figure 2. 
The assessment of model fit (the fit between the actual data and the estimated, hypothesized model) in MACS analyses is evaluated using either a statistical or a modeling rationale (Little, 2000). When a statistical rationale is used, equivalence across groups is tested as a nested-model comparison between a model in which specific parameters across groups are constrained to equality and one in which the parameters in all groups are freely estimated (Bollen, 1989).

Model fit is assessed by evaluating the difference in $\chi^{2}$ (Chi-square difference test) between the two models is a test of the equality restrictions and several overall model fit indices (i.e. TLI, CFI and RMSEA). Failure to obtain adequate fit suggests that either different groups produce different numbers of factors or some items load on different factors across groups, or both, which "fit" the data better than the hypothesized number of correlated common factors. If acceptable fit is not obtained, then an adequate baseline model does not exist and it makes little sense to continue testing for measurement equivalence (Bollen, 1989).

Hypothesis 2: Equality of Factor Loadings

Hypothesis 2 tests the fit of the equality of factor loadings, also referred to as factorial invariance. The baseline model is compared to a model in which all factor loadings are constrained to be equal across groups; that is

$$
\mathrm{H}_{\Lambda \mathrm{x}}: \Lambda_{\mathrm{x}}^{(1)}=\Lambda_{\mathrm{x}}^{(2)}=\ldots=\Lambda_{\mathrm{x}}^{(\mathrm{G})}
$$


for all groups (g). Model specification is the same for hypothesis 1, except for the addition that all the factor loadings $\left(\Lambda_{\mathrm{x}}\right)$ are constrained to be equal across the groups.

The constrained model is tested against (or compared to) the baseline model (hypothesis 1). Model fit assessment is the same as described in hypothesis 1 . The measurement model is depicted in Figure 3.

Factorial invariance indicates that the respondents across groups ascribe approximately the same weight to the indicators, as manifested by equal (i.e. not significantly different) factor loading parameters. This equivalence condition is most frequently of interest, because it is a necessary condition for comparisons across groups and for comparing means and intercepts in a latent variable system (Bollen, 1989).

If factorial invariance does not hold, (i.e. factor loadings are significantly different), subsequent tests are required to determine the sources of non-invariance that may be attributed, for example, to poorly translated items, or, in particular, extreme response set (ERS) bias, the tendency of one group to consistently use the extreme categories of the rating scale on particular items than another group.

However, factorial invariance will not detect a systematic response set bias, also referred to as acquiescence response set (ARS) (Cheung \& Rensvold, 2000) bias, which occurs when one group has a tendency to respond systematically higher or lower to the indicators than other group, resulting in scale displacement, even if both groups have the same factor loadings parameters (Bollen, 1989, Mullen, 1995). This response tendency 
would be detected in a difference in the intercepts for the same item of across groups (Bollen, 1989).

In sum, nonuniform ERS is related to unequal factor loadings, while nonuniform ARS is related to unequal intercepts (i.e. intercept noninvariance). These threats to the (cross-cultural) reliability and validity of scales are addressed in the hypotheses 3 , equality of intercepts.

Hypothesis 3: Equality of Intercepts

Hypothesis 3 tests the fit of the equality of intercepts or strong factorial invariance (Meredith, 1993; Little, 1997). The factor invariant model, hypothesis 2, is compared to a model in which all intercepts of the indicators are constrained to be equal across groups; that is

$$
\mathrm{H}_{\Lambda v}: \Lambda_{\mathrm{x}}^{(1)}=\Lambda_{\mathrm{x}}^{(2)}=\ldots=\Lambda_{\mathrm{x}}^{(\mathrm{G})}, \quad v_{i}^{(1)}=v_{i}^{(2)}=\ldots=v_{i}^{(\mathrm{G})}
$$

for all groups $(g)$. The equality of intercepts of each item, that is the value of the manifest variables (indicators) when the value of the latent mean is zero (0), tests for consistency in responses. Following Bollen (1989), the measurement equation to estimate intercept terms $\left(v_{x}^{(\mathrm{g})}\right)$, also involves estimates of the latent means $(\kappa)$, 


$$
x^{(\mathrm{g})}=v_{x}^{(\mathrm{g})}+\Lambda_{x}^{(\mathrm{g})} \xi^{(\mathrm{g})}+\delta^{\mathrm{g})},
$$

where the expected value of $x$ is

$$
E(x)=v_{x}+\Lambda_{x}+\kappa
$$

for all groups $(g)$. The first equation estimates the mean, $\kappa$, of the latent variables $(\xi)$ and the second equation estimates the intercepts of the manifest variables $(x)$. Convention requires at a minimum the equality of factor structures (hypothesis 1) and the equality of factor loadings (hypothesis 2) across groups before restrictions on intercepts and means can be tested (Bollen, 1989, p. 366).

Model specification is the same as hypothesis 2, except for the addition that all the intercepts $\left(v_{x}{ }^{(g)}\right)$ are constraint to be equal across groups. To identify the model, the latent means must be assigned a scale and origin (Bollen, 1989). Following Bollen (1989), each latent variable has its scale and origin matched to one of the observed variables $\left(X_{i}\right)$. This scale is established by setting the factor loadings $(\lambda)$ of an arbitrary observed variables to one (1) and the corresponding intercepts $\left(v_{x}\right)$ to zero $(0)$. This leads to $E\left(X_{i}\right)=\kappa_{j}$, where $\kappa_{j}$, is a single latent variable that underlies the manifest variables $\left(X_{i}\right)$. Thus, the latent variable is given the same mean and units as the observed variables $\left(X_{i}\right)$. Similar to the previous tests, the constrained model is tested against (or compared to) the lesser-constrained model ( $\mathrm{H}_{2}$ ). Model fit criteria are the same as the previous hypotheses. The measurement model is depicted in Figure 4. 
Intercept invariance, or strong factorial invariance (Meredith, 1993, Little, 1997)

indicates that the constructs have equivalent measurement properties (i.e. they are defined in the same operational manner in each group studied) and, thus, they can be compared meaningfully and with quantitative precision across the groups studied (Little, 1997, 2000). Thus, strong factorial invariance (i.e. intercept invariance) is a necessary condition for comparing differences in the constructs' (latent) means. As such, the cultural differences in the constructs' latent space are quantifiable in nature, and can be assessed as mean-level, variance, and covariance or correlational effects (Little, 1997; 2000).

If intercept invariance hypothesis is rejected, significant intercept differences point toward the presence of a differential bias and confound the detection of true mean differences on the latent variable (Cole, Maxwell, Arvey \& Salas, 1993). In simultaneously equated multiple latent variable model, separate intercept tests on each construct can be conducted first, before an attempt to compare the means of the latent constructs is abandoned (Cheung \& Rensvold, 2000). Separate intercept tests for initiation of structure and consideration are depicted in Figures 5 and 6.

Advocates of strong factorial invariance (i.e. Meredith, 1993; Cheung \& Rensvold, 2000; Little, 2000) contend that any test of factorial invariance must include the intercepts or means of the indicators. Strong factorial invariance is believed to be less biasing than strict factorial invariance (full scale equivalence) equivalence, in which measurement errors are constrained to be equal across groups, because even if random 
error is quite similar across groups, if it is not exactly equal, the non-equal part of the random error is driven into other parameters of the model and could introduce possible sources of bias. Under the assumption that such biases or errors are negligible, they should not be constrained across groups so that the theoretically meaningful common variance components can be analyzed for cross-cultural differences with as little bias as possible (for details see Little, 2000, p. 55, footnote 1)

Strong factorial invariance indicates construct comparability or measurement equivalence. A more restrictive condition is advocated by Mullen (1995), the equality of measurement error variances, also referred to as strict factorial invariance (Meredith, 1993; Little, 1997) or full scalar equivalence (van de Vijver \& Tanzer, 1997). The equality of measurement error variances is a combined test of the equality of systematic error variance and random error variance and addresses both the reliability and validity of measurement scales (Bollen, 1989; Mullen 1995).

Hypothesis 4: Equality of Measurement Error Variances

Hypothesis 4 tests the fit of equality of measurement error variances. The factor invariant model is compared with a model in which all measurement error variances are constrained to be equal across groups; that is

$$
\mathrm{H}_{\Lambda \Theta}: \Lambda_{\mathrm{x}}{ }^{(1)}=\Lambda_{\mathrm{x}}{ }^{(2)}=\ldots=\Lambda_{\mathrm{x}}{ }^{(\mathrm{G})}, \Theta_{\delta}{ }^{(1)}=\Theta_{\delta}{ }^{(2)}=\ldots=\Theta_{\delta}{ }^{(\mathrm{G})}
$$


for all groups $(g)$. Model specification is the same as hypothesis 2, factorial invariance, except for the addition that all measurement errors $\left(\Theta_{\delta}^{(G)}\right)$ are constraint to be equal across groups. Again, the constrained model is tested against (or compared to) the lesser constrained model (H 2) and, subsequently, evaluated by the same criteria as in the previous hypotheses. The measurement model is depicted in Figure 7.

If equality of measurement error variance hypothesis holds, measurement equivalence of the constructs is established. This combined test of systematic and random error variance addresses two prevalent threats to measurement equivalence, inconsistent scoring and scalar nonequivalence across populations (Douglas and Craig, 1983; Riordan \& Vandenberg, 1994; Triandis, 1994). First, inconsistent scoring results in random error (e), which affects the reliability of the scale (Mullen, 1985). This occurs when subjects in a particular culture are either unfamiliar with the scale's application or its scoring format, they may respond to it inconsistently, which threatens the reliability of the scale. Second, scalar nonequivalence or response set bias (Cunningham, Cunningham and Green, 1977; Mullen, 1995; Cheung \& Rensvold, 2000), may affect systematic error $(s)$, threatening the validity of the scale for cross-cultural comparison (Mullen, 1995). Here, the issue is whether the scores obtained from subjects in different countries have the same meaning and interpretation (Douglas and Craig, 1983). Scaling or response set bias may be due to cultural characteristics such as acquiescence, social desirability, or modesty, all of which may influence subjects scoring (Mullen, 1995). 
Measurement equivalence will hold if cultural influences have affected the common-variance component of a construct's indicators and not differently influenced the indicators unique components (Meredith, 1993, Little, 2000). If cultural influences affect the specific components of the indicators, such as when an item is perceived and responded to differently in one group, non-equivalence of the construct would appear. However, measurement equivalence does not rule out uniform construct-level biases Little, 2000). For instance, if acquiescence, social desirability and/or modesty are pervasive characteristics (i.e. defining aspects) of a cultural group, then they are part of the cultural makeup of the individuals. These characteristics, like any other defining characteristic, will affect each indicator of a construct to approximately to the same degree if the indicators are consistently representative of the construct (Little, et al., 1999). Therefore, the construct would, inevitably, reflect this influence (Little, 2000).

In sum, if pervasive cultural characteristics uniformly affect the responses of individuals, the construct's means and variances would be affected (Meredith, 1993; Little, 1997). At the construct level, uniform cultural influences are not measurement artifacts, but reflect characteristics of a particular group of individuals (Little, 1997, 2000). These cultural influences are quantitative in nature (i.e. between culture differences) and can be assessed as mean-level, variance, covariance or correlational effects (Little, 2000, p. 215). In hypothesis 5 the constructs means are tested across groups for evidence of pervasive cultural influences (i.e. between-group differences). 
Hypothesis 5: Equality of Latent Means

Hypothesis 5 tests the fit of equality of latent means. The intercept invariance model is compared with a model in which the latent means are constrained to be equal across groups; that is

$$
\mathrm{H}_{\Lambda v \mathrm{~K}}: \Lambda_{\mathrm{x}}^{(1)}=\Lambda_{\mathrm{x}}^{(2)}=\ldots=\Lambda_{\mathrm{x}}^{(\mathrm{G})}, \quad v_{i}^{(1)}=v_{i}^{(2)}=\ldots=v_{i}^{(\mathrm{G})}, \quad \kappa_{i}^{(1)}=\kappa_{i}^{(2)}=\ldots \kappa_{i}^{(\mathrm{G})}
$$

for all groups $(g)$. Model specification is the same as hypothesis 4 except for the addition that the latent means $\left(\kappa_{i}^{(G)}\right)$ are constraint to be equal across groups. Again, the constrained model is tested against (or compared to) the lesser constrained model ( $\mathrm{H} 4)$ by calculating the Chi-square difference test statistic $\left(\chi^{2}=\chi^{2}\right.$ constrained $-\chi^{2}$ unconstrined $)$ and the differences in several model fit indices (i.e. $\triangle \mathrm{TLI}, \triangle \mathrm{CFI}$ and $\triangle \mathrm{RMSEA}$ ).

The identification of the model follows Bollen (1989) and Arbuckle and Wothke (1999) and the AMOS 4.0 program modeling conventions and is identical to hypothesis 3. The difference in set up, however, is in the latent means, and requires a two-step process. First, the latent means of an arbitrary group (e.g. the U.S. in this study) are set to zero (0). By setting the means for the U.S. sample to zero, the model is identified, and the other means can be estimated. This method tests the relative means of the two latent variables (constructs) across the 11 samples, because all means cannot be estimated at once. The next step is to carry out a test of the null hypothesis that the latent means are equivalent across groups. To do this, the previous analysis will be repeated but with the 
added constraint that all 12 groups have the same latent means. Since the arbitrary group's latent means (here the U.S. sample) were fixed at zero in the previous step, in the subsequent step the latent means of the other groups will be constrained to zero, as well. The two measurement models in the two-step approach are depicted in Figures 8 and 9.

The two-step process assesses whether the latent means (parameters) predicted to be nonzero in the model are in fact significantly different from zero. To test whether there is a significant difference (in the latent means) from zero, the ratio of the latent mean to its standard error is reported as a $t$ test by the AMOS 4.0 program. Given the large sample size of this study, these $t$ values are in practice interpreted using the critical values for the $Z$ test, such that values above 1.96 are significant at the $p<0.05$ level (Kelloway, 1998, p. 29), said differently, values above 1.96 are significantly different from zero.

Nonequivalence of latent means may indicate that the differences in the constructs across groups are due to pervasive cross-cultural influences and not to measurement artifacts. Thus, when the latent means are estimated as the optimal common difference, and should nonequivalence occur, it reflects the true, valid (cross-cultural) differences across groups (McArdle \& McDonald, 1984; Little, 2000). The MACS analytical system allows for precise tests of cultural differences in a quantitative manner, while simultaneously establishing measurement equivalence (Little, 2000). Data analysis and results are discussed in the next chapter. 


\section{DATA ANALYSIS AND RESULTS}

The preceding chapter described the MACS analyses framework and hypotheses tests. This chapter reports the data collection techniques and the results from the empirical analyses of the data.

Data analyses are performed in three stages. First, a demographic profile for each country is investigated for sample comparability. During the second stage, the psychometric adequacy of the two leadership scales, consideration and initiation of structure, is examined for possible threats to the assumptions of multivariate normality that underlie MACS analyses. The occurrence of item bias, a major problem in crosscultural research, is also probed during this stage. In the third stage the hypotheses are tested in MACS analyses.

\section{Data Collection}

Sample

This study uses data collected by this researcher and others as part of a larger research project, the Best International Human Resource Management Practices project (i.e.Best Practices Project), which was designed to examine a range of international 
human resource management practices, leadership and organizational contextual factors across countries. A detailed discussion of the genesis of the "Best Practices" project and its methodology is provided by Teagarden, et al. (1995).

The questionnaire was distributed to both managers/engineers and non-managers in some countries and to only managers/engineers in other countries. In an effort to improve sample comparability, only manager data were included in the analyses reported in this study. Significant differences in the samples remain in the depth and breadth of industries surveyed in each country. Though differences in sample comparability with respect to type of industry are common to comparative international research, caution remains prudent in interpreting the results (Milliman, Nathan, Von Glinow, Huo, Lowe, and Kim, 1995). In general, personal and academic contacts were used for data collection, which means a shift from a random sampling technique to one of quasitheoretical based sampling.

The research samples in this study are comprised of 2341 managers and engineers from 10 countries and two regions. The number of respondents completing the survey, average age, percentage that are male, and percentage with a bachelor's degree or higher education level respectively are as follows: Australia ( $n=438,36$ years, $58 \%$ male, $67 \%$ bachelor's or higher), Canada ( $\mathrm{n}=126,41$ years, $81 \%$ male, $75 \%$ bachelor's or higher), USA ( $n=145,41$ years, $64 \%$ male, $87 \%$ bachelor's or higher), China ( $n=192,36$ years, $67 \%$ male, $66 \%$ bachelor's or higher), Taiwan ( $\mathrm{n}=118,36$ years, $86 \%$ male, $84 \%$ bachelor's or higher), Japan ( $\mathrm{n}=280,37$ years, $92 \%$ male, $70 \%$ bachelor's degree or 
higher), Korea ( $\mathrm{n}=242,32$ years, $98 \%$ male, $71 \%$ bachelor's or higher), Indonesia $(\mathrm{n}=247,82 \%$ male, $77 \%$ bachelor's or higher), Philippines ( $\mathrm{n}=168,44$ years, $43 \%$ male, 98\% bachelor's or higher), Gulf region (Jordan and Saudi Arabia ) $(n=108,60 \%<30$ years, $79 \%$ male, $72 \%$ bachelor's or higher), Latin America ( $n=145,33$ years, 59\% male, $81 \%$ bachelor's or higher), and Mexico ( $\mathrm{n}=185,32$ years, $80 \%$ male, $77 \%$ bachelor's or higher). Demographic profiles for each country/region are reported in the Appendix.

Questionnaire

The questionnaire was originally developed for a large international research project, the Best Practices in International Human Resources Project, in which this researcher has contributed. The questionnaire included the 10 -item short form version of the 20-item LBDQ-XII (Stogdill, 1963) to measure consideration and initiation of structure. Earlier research identified the LBDQ-XII as the most reliable measure of consideration and initiation of structure (Schriesheim and Kerr, 1974). Also included in the questionnaire were items on human resource management practices, communication, business strategy practices, cultural factors, organizational contextual factors, job satisfaction, organizational effectiveness and demographical data.

The questionnaire was developed in English and translated into various languages of the participating countries by native-born bilingual MBA students and/or bilingual researchers familiar with the business environment in that country. Subsequently, back translations were performed by native born professors from the management field. 
Given the constructs established validity with U.S. samples (Schriesheim \& Kerr, 1974) and cross-national samples (Tscheulin, 1973), a 10-item short form version of the revised LBDQ-XII (Stogdill, 1963) was designed to operationalize the two factor model of consideration and initiation of structure. This shorter version included 5 items for each construct. For this study, only 9 items were included, because item 6 ("stresses high standards of performance for group or unit") appeared to duplicate item 2 ("emphasizes high standards of performance"), and rendered difficulties with conceptualization and translation in certain cultures. This resulted in a 5-item scale for consideration and a 4-item scale for initiation of structure.

The 9 leadership items were measured with a 5-point Likert-type scale with the anchors $1=$ Not at all, $2=$ To a small extent, $3=$ To a moderate extent, $4=$ To a large extent, 5=To a very great extent. The 9 leadership items queried the extent to which the respondent's immediate supervisor exhibited consideration and initiation of structure: My immediate supervisor; 1) sets specific goals for me to accomplish; 2) emphasizes high standards of performance; 3) stresses the importance of work goals; 4) is friendly and easy to approach; 5) is eager to recognize and reward good performance; 6) stresses high standards of performance for group or unit (this question was dropped from the scale due to difficulties with conceptualization in translation); 7) is willing to listen to my problems; 8) treats me with respect; 9) checks everything, individual judgment is 
not trusted; 10) when suggestions are made to top management, they receive fair evaluation.

Respondents were asked two types of questions when describing their immediate supervisor's behavior: first, to indicate the current behavior of their supervisor (is now), and second, to indicate the desired (ideal) behavior of their supervisor (should be). A total of 18 responses were made to the 9 items, 9 is now assessments, and 9 should be assessments. For this study, the current state (is now) is considered to establish measurement equivalence for the two leader behaviors. The set of demographic variables and leadership items, including their instructions, is provided in the Appendix.

\section{Preliminary Data Analyses}

Demographic Profile Analyses

The demographic profile variables (i.e. gender, age, education, and salary) for each country and region were examined through various SPSS 10.0 techniques (e.g. frequencies, cross tabulations) for accuracy of data entry, missing values, and fit between their distributions and the assumptions of multivariate analysis. The demographic variables were also examined for male and female managers, separately, and are reported in the cross-tabulations in the Appendix. 
Cases with an out-of-range value (e.g. data entry error) were treated as missing values. In 4 countries, U.S., China, Latin America and the Philippines, data entry errors in gender classification were also treated as missing values. The salary classifications in the Mexico data were adjusted and recoded to fit the general salary classifications (to US dollars) of the other samples. After screening and cleaning each data set, the data were merged into one large data set (spreadsheet) to conduct the preliminary and statistical analyses.

Overall response rates were 76 percent male and 24 percent female across the 12 cultures sampled. Most of the respondents were between the age of 30 and 50 (74\%) and well educated, with the majority $(73 \%)$ having at least a bachelors degree. The salaries of the managers were distributed quite evenly across the five salary levels, ranging from less than $\$ 25,000$ to more than $\$ 100,000$ USD. The respondents' average company and job tenure were 10 and 5 years, respectively.

Although the sample as a whole appeared quite homogeneous with respect to age and education, differences were apparent. First, and importantly, the data were underrepresented by female managers. The U.S., China, Latin America and Australia were the most closely matched with regard to the percentage of female managers with $35 \%, 33 \%$, $39 \%$ and $34 \%$, respectively. The Philippines had the highest percentage of $58 \%$ female managers, and Japan and Korea had the lowest percentage of female managers, 7\% and $1 \%$, respectively. 
Gender differences in educational background were also noted. Japan, Korea, Australia and China had the largest percentages of male managers with less than a college education with $45 \%, 38 \%, 31 \%$ and $24 \%$, respectively, in comparison to other male managers in the sample with less than a college education. The largest percentage of non-college educated female managers were reported for Australia, China and Canada with $58 \%, 49 \%$ and $45 \%$, respectively, in comparison to other female managers in the sample with less than a college education. The largest within-country differences in education level for both male and female managers were given for China, Japan and the Gulf region.

Salaries were the lowest for managers from Mexico, the Philippines and the Gulf region with earnings of less than $\$ 25,000$ per year. For male managers these percentages were $47 \%, 73 \%$ and $78 \%$, respectively. For female managers these percentages were $93 \%, 83 \%$ and $75 \%$, respectively.

Age differences between male and female managers were also observed. The largest percentages of female managers under 30 years of age were observed in the Gulf region, Korea, Mexico, Latin America and Taiwan with $67 \%, 67 \%, 45 \%, 39 \%$, and $31 \%$, respectively. The Gulf region showed the largest percentage of male managers (58\%) under 30 years old, followed by Indonesia with $21 \%$ of male managers under 30 . 
The 9 leader behavior items were examined for accuracy of data entry, missing values, and fit between their distributions and the assumptions of multivariate analysis through various SPSS 10.0 techniques for the total sample and each country, separately. Descriptive statistics (i.e. means, standard deviations, skewness and kurtosis) for 9 leader behavior items for the total sample and for each country /region, separately, are reported in the Tables 3 and 4.

The distributional properties of the 9 leader behavior items indicated moderate skewness and kurtosis in the 12 data sets. According to Bollen (1989), the consequences of the violations of the multivariate normality distributional assumptions on Maximum Likelyhood (ML) estimators are mainly reflected in the robustness of the model estimates and tests of statistical significance. Boomsma (1983) observed that with high skewness, the chi-square tended to be too large. In another study, Muthen and Kaplan (1985) compared of various estimators, among others, ML and general least squares (GLS), of factor analysis for non-normal Likert-type scales and observed that ML and GLS chisquare tests and estimated standard errors were relatively robust, except when the observed variables had large skewness or kurtosis. According to Muthen and Kaplan (1985), not much distortion will occur with ML and GLS estimators when skewness and kurtosis range from $-1,0$ to +1.0 . West, Finch and Curran (1995) recommended a wider skewness and kurtosis range (skew $>2$; kurtosis $>7$ ) for questioning the adequacy of ML estimation methods than Muthen and Kaplan (1985). 
Because most of the observed skewness (-1.68 to 1.12$)$ and kurtosis $(-1.49$ to 2.64) in the data are within the Muthen and Kaplan (1985) and West et al.'s (1995) recommended range, the data were not standardized to correct for the assumptions of multivariate normality.

At the country level, the distributional properties of all items in the Gulf data indicated high negative kurtosis $(-0.747$ to -1.315$)$. The Canadian data revealed both high positive skewness and kurtosis (1.266 and 1.173, respectively) for item 9, (my immediate supervisor checks everything, individual judgment is not trusted). At the item level, the distributional properties of item 8, (my immediate supervisor treats me with respect), were high in skewness and kurtosis in the Australia, Canada, Mexico, Latin America and high negative skewness in the Philippines data sets.

The distributional properties of the total sample indicated mild negative skewness and kurtosis for all items. However, the range of negative skewness $(-0.008$ to -0.498$)$ and negative kurtosis $(-0.260$ to -0.835$)$ of the items were much smaller than the recommended thresholds for questioning the adequacy of ML (Maximum Likelihood) estimation methods (see West et al., 1995), which will be used (e.g. Joreskog, 1981; Bollen, 1989) in the MACS analyses. 
The differences in the distributional properties of the 9 items across the 12 samples, initiated an investigation in item bias, before subsequent construct level analyses were performed. Because gender differences are frequently reported in crosscultural research (e.g. Gorphade, Hattrup \& Lackritz, 1999), the data were first examined for gender differences. Analysis of variance (ANOVA) and between-subjects multivariate analysis of variance (MANOVA) were conducted for each country/region, separately, to identify whether differences in the managers' mean rating on the items were due to cross-cultural differences, gender differences or both in the 12 countries/regions studied. The results of the MANOVAs are reported in the Appendix.

A between-country (groups) ANOVA indicated significant differences in the managers' average ratings/responses for all (9) items across the 12 countries/regions. Next, an investigation of gender differences of the total sample revealed significant gender differences in mean responses to three items: items 2, (my immediate supervisor emphasizes high standards of performance); item 4, (my immediate supervisor is friendly and easy to approach); and, item 7, (my immediate supervisor is willing to listen to my problems), all three at the $\mathrm{p}<.05$ level. Inspection of the stem-and-leaf plots and box plots for these data indicated that the ratings of the female managers' on these 3 items were higher than their male counterparts. 
The within country ANOVAs did not indicate significant gender differences in the managers average ratings/responses to the 9 items in the U.S., Canada, Australia, the Gulf region and the Philippines data. Minor gender differences were observed in Indonesia, the Latin American region and Taiwan; item 2, (my immediate supervisor emphasizes high standards of performance); item 8, (my immediate supervisor treats me with respect); and, item 1, (my immediate supervisor sets specific goals for me to accomplish), for each country, respectively, at the $\mathrm{p}<0.05$ level. Three items indicated a gender difference in Mexico: item 2, (my immediate supervisor emphasizes high standards of performance) at the $\mathrm{p}<0.05$ level; item 4, (my immediate supervisor is friendly and easy to approach); and, item 3, (my immediate supervisor stresses the importance of work goals), both at $\mathrm{p}<0.10$ level.

Major gender differences in average scores on the 9 items were observed in the Chinese and Japanese data sets. Most of the items indicated significant differences at both the $p<0.05$ and $p<0.10$ levels. However, the Japanese and Korean data sets included only 19 and 3 female managers, respectively. These results are, therefore, not representative of the data set and need to be interpreted with caution.

The between-subjects MANOVA indicated a significant interaction between gender and culture on all of the items with the exception of item 9 ("my supervisor checks everything) and item 10 ("when suggestions are made to top management, they receive fair evaluation). These results suggest the ratings on 7 items are influenced by gender, varying by culture. However, the results of ANOVA require full score 
equivalence (van de Vijver \& Leung, 1997). When the presence of bias cannot be ruled out, the interpretation of significant differences may be ambiguous. Because the data are underrepresented by female managers, gender effects are to be expected.

Item Bias Detection Analysis

Next, an item bias analysis on the 9 items was performed to further screen the data for possible threats to the assumptions of multivariate normality. The results are reported in the Appendix.

The item bias detection technique recommended by van de Vijver and Leung (1997), which was used in this study, applies between-subjects MANOVAs. The item score is the dependent variable and the 12 cultural groups and the score levels-9-15,1620,21-25,26-30,31-35,36-40,41-44-are the independent variables. The analysis is conditional because score level is used in the design specification as an independent variable (van de Vijver \& Leung, 1997). When both the main effect of culture (country/region) and the interaction between score level and culture is non-significant, the items are considered to be unbiased (van de Vijver \& Leung, 1997). A significant main effect of culture indicates uniform bias, which implies that one group may have higher scores on an item than individuals from another cultural group even when they have the same total test score (van de Vijver \& Leung, 1997). A significant interaction between score level and culture indicates that the difference across cultural groups is not invariant (equivalent) across score levels. That is, the item discriminates better in one 
group than in another, which is referred to as non-uniform bias (Mellenbergh, 1982; van de Vijver \& Leung, 1997).

The data were divided into score groups on the basis of the total score of the instrument. The minimum score on the Likert-type scale in this study was $9 \times 1=9$ and the maximum score was $9 \times 5=45$. The minimum and maximum score groups were not considered. The remaining score groups (10 through 44) provided valuable information for item bias analysis. Because it was infeasible to separate all possible score groups (most of the levels will have insufficient data to warrant such an analysis), the data were divided into the appropriate number of score levels. The score widths of the groups were chosen in such a way that the number of subjects in the groups were as similar as possible. For this study, 7 score groups with approximately 350 persons each seemed appropriate.

Inspection of the distribution of the 7 score levels indicated a normal distribution. However, a frequency analysis indicated 50 entries with a total item score of zero $(0)$. After inspection of the data spreadsheet, it was discovered that 50 cases did not have any scores for the leader behavior items on the questionnaire. After double-checking with the preliminary data sets, the 50 cases were eliminated from the sample.

The results of the MANOVA indicated a main effect of culture for all items with the exception of item 5 (my immediate supervisor is eager to recognize and reward good performance). Consequently, certain groups may have higher or lower scores on an item 
than individuals from another group, even though they have the same total test score (van de Vijver, 1997). Moreover, 3 nonuniform scoring biases were detected for items: item 2, (my immediate supervisor emphasizes high standards of performance); item 8, (my immediate supervisor treats me with respect); item 9, (my immediate supervisor checks everything; individual judgment is not trusted), which could indicate that these items discriminate better in one group than in another (Mellenbergh, 1982; van de Vijver \& Leung, 1997). Separate analyses were run for male and female managers which indicated similar interaction effects between culture and score level (non-uniform bias) for both groups, with the exception of item 3, (my immediate supervisor stresses the importance of work goals), which indicated a significant interaction in the female sample, only.

In sum, the item bias analysis indicated that managers did not respond differentially to the leader behavior items, but that managers from certain groups may have higher or lower scores on an item than individuals from another group, even though they have the same total test score (van de Vijver \& Leung, 1997). However, interaction effects in ANOVA are recognized for their cross-sample instability (van de Vijver \& Leung, 1997), which could have disturbed the nonuniform item bias detected in the three items referred to above. The psychometric properties of the 9 items are examined in the next section. 
The Cronbach alpha reliability coefficient was used to assess the intercorrelations among the 9 items underlying the two constructs. The alpha coefficients for consideration and initiation of structure are reported for each country and the total sample in Table 5. The correlations among the 9 items for the total sample are reported in Table 6. Correlations among the 9 items for each country are reported separately in the Tables 7 through 18.

Overall, the managers reported a fairly strong sense of consideration and initiation of structure. The 9 measures attained adequate reliabilities (i.e. $>0.70$, see Nunnally, 1978) ranging from 0.72 to 0.90 in most of the countries/regions sampled. Low alphas were observed in Australia for consideration with an alpha score of 0.50, and in Canada, the U.S. and Indonesia for initiation of structure with alphas scores of $0.59,0.64$, and 0.043 respectively.

The correlation matrix for the Australian sample indicated negative correlations between item 8, ("my immediate supervisor treats me with respect"), and all other items. Moreover, the U.S. and Canadian samples indicated negative correlations between item 9, ("my immediate supervisor checks everything: individual judgment is not trusted"), and all other items. The negative correlations most likely contributed to the lower reliabilities of both constructs in the Australian and U.S samples. The Indonesian correlation matrix was difficult to interpret. Item 1 revealed extreme low correlations 
with all other items (range 0.016 to 0.115 and -0.148 ). Items 2,3 and 10 indicated somewhat higher correlations with the other items (range 0.091 to 0.278 ). Items $4,7,8$ and 9 indicated high intercorrelations (range 0.47 to 0.73 ). The pattern of correlations ain the Indonesian data may indicate that the managers perceived consideration and initiation of structure differently than their intended meaning, perhaps contributing to the low reliability for the initiation of structure construct.

By and large, the 9 items indicated high, positive correlations for most of the countries/regions sampled. However, high positive intercorrelations among the measures for consideration and initiation of structure and between the two constructs have historically been reported (e.g. Bass, 1990; Fleishman, 1998; Schriesheim, Cogliser \& Neider, 1998).

\section{Results of Preliminary Data Analyses}

The initial demographical analysis indicated an underrepresentation of female managers (24\%), in particular in the Japanese and Taiwanese samples. The distributional properties of the 9 leadership items indicated moderate skewness and kurtosis across the 12 groups. Multivariate between-subjects tests indicated a significant interaction between gender and culture suggesting that the ratings of the managers were influenced by gender, varying across cultures (van de Vijver, 1997). Although the distributional distortions and gender differences were of concern, the distortions in the data were within the norms of multivariate normality for Maximum Likelyhood (ML) estimators (e.g. 
Muthen \& Kaplan, 1985; West, Finch \& Curran, 1995). The Cronbach alpha reliability coefficients for the two constructs were acceptable (i.e. $>0.70$, see Nunnally, 1978) for most countries/regions. The Indonesia data's low alpha for initiation of structure $(0.28)$ and ambiguous correlation matrix made this data questionable. However, the Indonesia data were included in the multiple-group analyses, because the ML estimator is fairly robust to the violation of the multivariate normality (Muthen \& Kaplan, 1985). The results of the multiple-group MACS analyses are discussed next.

\section{Hypotheses Tests}

The multiple group MACS analyses were executed by AMOS 4.0 (analysis of moment structures, developed by the Smallwaters Corporation and distributed by SPSS) to test the hierarchy of hypotheses discussed in the previous chapter. AMOS implements the general approach to data analysis known as structural equation modeling (see AMOS 4.0 Guide, Arbuckle \& Wothke, 1999).

Consideration and initiation of structure, were tested for measurement equivalence and, subsequently, for possible cross-cultural differences in the constructs' (latent) means across 12 countries/regions. A covariance matrix for each country/region was used as input for model evaluation using the Maximum Likelihood (ML) estimation procedure recommended by Bollen (1989). To date, the ML is the most widely used fitting function for general structural equation models. One of its major advantages is that its properties are asymptotic so that they hold in large samples (see Bollen, 1989, p. 
107-112). A tabular summary of the results is presented in Table 19, which includes statistics for $\chi^{2}$, df, $\Delta \chi^{2}, \Delta \mathrm{df}, \mathrm{CFI}, \mathrm{TLI}, \Delta \mathrm{TLI}, \mathrm{RSMEA}, \Delta \mathrm{RSMEA}$ and Pclose as they apply to the sequence of hypotheses tests. Additional AMOS 4.0 model fit indices for each hypothesis test are reported in the Appendix. The complete AMOS 4.0 output for each model may be obtained from the author.

Hypothesis 1: Same Factor Structure

Hypothesis 1 tested whether the baseline model for consideration and initiation of structure could be applied across cultures. The initial results indicated that the covariance matrices for Indonesia were not positive definite (i.e. its implied matrices were not valid solutions). Consequently, the Indonesia sample was not included in the multiple group analyses.

The results indicated that the baseline model effectively captured the two leader behaviors across the remaining 11 countries/regions sampled. The fit indices, CFI 0.987 , TLI 0.978, RSMEA 0.034 and a Pclose of 1.000, indicated excellent model fit. Even though the $\chi^{2}$ value of 1117.814 with 312 degrees of freedom was highly significant, it was disregarded due to the statistic's well-known sensitivity to numerous constraints and large sample size (here $\mathrm{N}=2388$ ) (e.g. Medsker, Williams \& Holahan, 1995). Factor form equivalence allowed for subsequent tests in the equivalence hierarchy. 
Hypothesis 2: Equality of Factor Loadings

Hypothesis 2 tested whether the factor loadings for both constructs were invariant across the 11 groups. To assess the significant differences between the constrained model and the baseline model the following model fit criteria were used: $\Delta T L I$ less than or equal to .05 (Little, 1997), a significant $\triangle \mathrm{RSMEA}$ with a probability of close fit (Pclose) less than or equal to 0.05 (Browne \& Cudeck, 1993). These criteria were also used to assess model fit in all subsequent hypotheses.

Hypothesis 2 indicated an excellent fit of the model to the data. As seen in Table 19, the fit criteria, $\triangle$ TLI of $<0.05, \triangle$ RMSEA of 0.004 with a non-significant Pclose of 1.000 and an overall model fit of CFI 0.979 , were all within the recommended norms discussed earlier. The Chi-square difference test $\left(\Delta \chi^{2}=1472.794\right.$, df 356) was not taken as a reliable indicator due to its sensitivity to sample size (Cheung \& Rensvold, 2000).

Hypothesis 3: Equality of Intercepts

Hypothesis 3, the test for intercept equality or strong factorial invariance, indicated a marginal fit between the factor invariant model and the (equality of intercepts) constrained model. As seen in Table 19, the RSMEA 0.057 indicated a significant Pclose of 0.000 , however, the $\triangle T L I$ of 0.035 and an overall model fit index CFI of 0.940 were both within the recommended norms. 
Because the model indicated a marginal fit to the data, each construct was tested separately for intercept invariance. As seen in Table 19, initiation of structure indicated good model fit; all criteria were within the recommend norms (i.e. $\triangle T L I$ of 0.014 , a RSMEA of 0.047, a nonsignificant Pclose of 0.991, and overall model fit of CFI 0.965). Consideration indicated marginal fit as a result of a RSMEA of 0.053 and a significant Pclose 0.001 , however, the $\triangle T L I$ of 0.026 and overall model fit of CFI 0.945 were both within the recommended norms for model fit. The significant Chi-square difference tests ( $\Delta \chi^{2}$ tests: full model, $2283.314, \Delta$ df 90 ; initiation of structure; $2872.814, \Delta \mathrm{df} 40$ ) were not taken as reliable indicators due to their sensitivity to sample size (Cheung \& Rensvold, 2000).

For the most part, hypothesis 3 provided reasonable evidence that both consideration and initiation of structure were measurement equivalent under the condition of strong factorial invariance (Meredith, 1993; Little, 1997).

Hypothesis 4: Equality of Measurement Error Variances

Hypothesis 4, the test for equality of measurement error variances or strict factorial invariance (Meredith, 1993), demonstrated excellent model fit. As seen in Table 19, the fit criteria, $\triangle$ TLI $0.016, \triangle$ RSMEA 0.005 with a nonsignificant Pclose of 0.649 and an overall model fit of CFI 0.955 , were all well within the recommended norms. 
Overall, the model fit criteria for hypotheses 1 through 4 indicated reasonable fit between the freely estimated model and the measurement equivalent models. Thus, on the basis of a modeling rationale, consideration and initiation of structure were measurement equivalent in the 11 cultural groups sampled. The empirical support for each hypothesis tested was based on model fit indices. Measurement equivalence is a necessary condition for comparing differences in the constructs' means.

Because measurement equivalence was established, particular hypotheses about possible cultural influences on the construct can be meaningfully tested on the reliable and true properties of the constructs (i.e. the constructs' means, variances, covariances or correlations) (Little, 1997, 2000). Construct level hypotheses are tested under the strong factorial condition advocated by Meredith (1993) and Little (1997,2000), because if cultural influences and the unique factors of the indicators are independent when conditioned on the common variance components of the constructs, then an equivalent measurement space can be specified so that the common variance components of the constructs contain information about cultural influences. Strong factorial invariance assumes that sources of bias and error are negligible (see footnote in Little 1997, page 55) and, therefore, should be represented as unconstrained residual variance terms across groups in order to examine the theoretically meaningful common-variance components with as little bias as possible (Little, 1997).

In the next hypothesis, the (latent) means of consideration and initiation of structure are tested to uncover similarities and differences in the managers' average 
perception of consideration and initiation of structure (i.e. task-oriented and relationshiporiented behavior) across the 11 cultural groups.

Hypothesis 5: Equality of Latent Means

Hypothesis 5 tested for significant differences in relative means in a two-step model. The model predicted whether the latent mean parameters predicted to be nonzero in the model were in fact significantly different from zero. In other words, the predicted model parameters that are significantly different from zero represent are true differences on the latent means (i.e. managers' average perception) and not measurement artifacts. The unstandardized parameters (model output) for consideration and initiation of structure are given for each country/region in Figures through 10 through 31 . The relative estimated mean difference scores with their respective critical $t$ values for each construct are reported by country/region in Table 20 .

The constrained model (equality of latent means) was tested against hypothesis 4 , intercept invariance, as recommended by Meredith (1993), Little (1997), and Cheung \& Rensvold (2000). The model indicated a marginal fit to the data. As seen in Table 19, the RSMEA (0.054) was larger than 0.05 and the $\triangle$ RSMEA of 0.006 was significant (Pclose 0.000 ). However, the TLI of 0.939 and a $\triangle T L I$ of 0.021 and overall model fit with a CFI of 0.947 , indicated an acceptable fit to the data (Little, 2000). Therefore, on the basis of a modeling rationale, it can be concluded that the observed (similarities and) differences in the latent means of consideration and initiation of structure are real differences rather 
than measurement artifacts. As seen in Table 20, most of the constructs' means were significant (i.e. significantly different from zero), indicating that certain aspects of consideration and initiation of structure may be affected by the cultural influences.

The estimated mean difference scores for initiation of structure were significantly different from zero in only three countries/regions: Taiwan, 0.287 ( $p$ value 3.220 ), the Gulf region, -0.635 ( $p$ value -5.298 , and Mexico 0.227 ( $p$ value 2.391). The estimated mean difference scores for consideration indicated significant differences (from zero) 8 of the countries/regions: China -0.309 ( $p$ value -2.847$)$, Japan -.327 ( $p$ value -3.337 ), Korea -.720 ( $p$ value -6.947), Philippines 0.333 ( $p$ value 3.012 ), Gulf -0.547 ( $p$ value 4.033), Latin America 0.435 ( $p$ value 3.951) and Mexico 0.255 ( $p$ value 2.354).

\section{Results}

Hypotheses 1 through 4 provided powerful evidence that task orientation and relationship orientation, can be reliably and validly operationalized through consideration and initiation of structure and are, therefore, generalizable across the 11 cultural groups sampled. Specifically, "the constructs are defined in exactly the same operational manner in each group, and as a result, they can be compared meaningfully and with quantitative precision" (Little, 1997, p. 56). But, hypothesis 5 revealed significant differences in the estimated mean difference scores for both constructs, indicating that some aspects of these behaviors may be affected by cultural influences that characterize each cultural group. 
Estimated Mean Difference Scores in Task Orientation (Initiation of Structure)

As seen in Table 20, the estimated mean difference scores were not significantly different from zero in 8 of the 11 countries, namely, Australia, United States, Canada, China, Japan, Korea, Philippines and Latin America. In these countries, managers applied more or less equal weights to task-oriented behavior. The consistency in this pattern begins to support some form of universality in the application of task orientation among the managers in these 8 countries/region.

However, as seen in Table 20, the estimated mean difference scores in Taiwan and Mexico $(0.287$, CR $3.294 ; 0.227$, CR 2.397$)$ were significantly higher as compared to the other 9 countries/regions, suggesting that in Taiwan and Mexico managers believed task orientation to be far more important as compared to the managers from the other countries. As it relates to Taiwan, these results are similar to those reported by Bond and Hwang (1986) in their review of leadership studies in Taiwan.

The literature on leader behavior in Latin America and Mexico is rather ambiguous, in particular as it relates to task orientation in Mexico (e.g. Von Glinow \& Drost, 1998). For example, in a study of leader behavior in Mexico, Von Glinow and Drost (1998) observed that managers and non-managers often embedded task orientation in relationship orientation. Given Mexico's high collectivism (Hofstede, 1991), paternalism and rigid social structure, Drost and Von Glinow (1998) reasoned that leader behavior in Mexico reflected the paternalistic mind-sets of the Mexican managers- 
perhaps suggesting that task-orientation is an established practice-because managers were expected to simultaneously express considerate behavior and delegate tasks to subordinates. The results here suggest that task orientation is a distinct leadership practice in both Mexico and Latin America, lending some support to the effectiveness of directive leadership practices in Latin America (e.g. Bass, et al., 1979; Dorfman, et al. 1997).

As seen in Table 20, the estimated mean difference score in the Gulf region $(-0.635, \mathrm{CR}-5.298)$ was significantly lower as compared to the estimated mean difference scores in the other countries/region, indicating that the Gulf region manager are far less task orientated as compared to the managers in the other 10 countries/region. These results are not consistent with the, albeit limited, literature on leadership practices in the Middle East. For instance, in a comparative study of leadership styles in Turkey and the U.S., Marcoulides and Yavas (1998) found that the Turkish managers preferred a directive leadership style to a participative leadership style. Considering the region's high power distance (Hofstede, 1991) and commitment to the Islam, obedience and respect for one's superior are traditional societal norms, and, as a result, one would expect task orientation to be highly valued managerial practice in the Middle East (Scandura, Von Glinow and Lowe, 1999). These values may have affected the mind-sets of the Gulf region managers-perhaps indicated by the lower ratings on task orientation - because they believe task-oriented behavior to be usual (i.e. routine) rather than effective. 
Estimated Mean Difference Scores in Relationship Orientation (Consideration)

As seen in Table 20, the estimated mean difference scores in relationship orientation were significantly different for most countries/regions, with the exception of the Gulf region and the Philippines. However, these differences in relationship orientation also revealed some form of consistency within three cultural clusters, namely, the Asian cluster (i.e. China, Korea and Japan), the Latin American cluster (i.e. Latin America and Mexico, and the Anglo cluster (i.e. Australia, Canada and the U.S.). As seen in Table 20, the estimated mean difference scores in China, Japan, and Korea $(-0.309, \mathrm{CR}-2.847 ;-.327, \mathrm{CR}-3.337 ;-0.720, \mathrm{CR}-6.947$, respectively) were significantly lower as compared to the estimated mean difference scores in the Anglo and Latin American cultural clusters, whereas the estimated mean difference scores in the Latin American cluster were significantly higher compared to the Asian and Anglo cultural clusters.

As it relates to the Asian cultural cluster, there is an abundance of evidence in the literature that supports relationship-oriented behavior to be the norm rather than the exception in China, Japan and Korea (e.g. Bass, 1990; Bass, Burger et al. 1979; Chan, 1995; Dorfman \& Howell, 1998; Dorfman \& Howell, 1994; Hofstede, 1980; Hofstede, 1991). The Confucian value system, which permeates the Far Eastern societies represented in this study, emphasizes the welfare and development of subordinates, group harmony and conflict-free interpersonal relations (Steers, Shin \& Ungson, 1989). Even though Confucian values emphasize harmony, it is based on inequality, power and 
prestige (Alston, 1989). These values result in leaders with considerable power to direct subordinates, who, as a consequence, expect harmony (consideration) and accept direction (task structure). The lower ratings by the Asian managers seemed curious at first, considering the Confucian value system. For the Asian managers, relationship orientation may be so ingrained in their business practices, perhaps reflecting the traditional nature of harmony in their lower ratings on this behavior. However, as seen in Table 20, the results also indicated that relationship orientation is perceived differently within the Asian cultural cluster. For example, in Korea the managers applied far less weight to relationship orientation than did the managers in China and Japan, perhaps revealing the strong authoritarian style of superiors in managerial processes in Korean organizations (Chen, 1995, p. 217).

As it relates to the Latin American cultural cluster, the Latin American and Mexican managers perceived relationship orientation to be far more important as compared to the managers from the other countries represented in Table 20. Again, the literature on relationship orientation in Latin America and Mexico is confusing (see Drost \& Von Glinow, 1998). For instance, Burger, Doktor and Barrett (1979) concluded that Latin American managers regarded being relationship-oriented (considerate) as unimportant at all levels of management. In a study of leader behavior in Mexico, Drost and Von Glinow (1998) found task orientation was frequently embedded in relationship orientation and, therefore, it was not clear under which conditions these behaviors prevailed. The results here indicated that relationship orientation was indeed believed to be a distinct, important leader behavior. Again reflecting strong paternalism is the 
prevalence of relationship over task-perhaps suggesting a willingness to accept structure by subordinates - revealed by the high ratings of the Latin American and Mexican managers on relationship-oriented behavior.

To date, little is known about leadership practices in the Philippines. As seen in Table 20, the Philippines managers indicated a strong commitment toward relationship orientation in their leadership practices. Given the country's high collectivism (Hofstede, 1991), relationship-oriented behavior is expected to be highly valued in organizations in the Philippines.

As seen in Table 20, the Gulf region's estimated mean difference score was significantly lower as compared to the estimated mean difference scores in the other countries. This result suggests that in this region managers believe relationship orientation to be far less important as compared to the managers from all the other countries represented in Table 20. Similarly, in a study of leader behavior in the Middle East, Scandura, Von Glinow and Lowe (1999) found no relationship between relationship orientation and job satisfaction and leader effectiveness. The lower ratings observed in this study may well reflect the high power distance (Hofstede, 1991) mind-sets of the Gulf managers, which was alluded to earlier-perhaps indicating an unwillingness to accept relationship-oriented behavior-because they believe such behavior to be confusing to subordinates and expose weakness and/or uncertainty on the part of the leader (Hofstede, 1992; Scandura, Von Glinow and Lowe, 1999). 


\section{DISCUSSION AND CONCLUSION}

The major premise of this study was to hypothetically develop and empirically confirm the universal application of task orientation and relationship orientation and the generalizability of their measurement across cultures. The lack of empirical evidence supporting such an application provided the impetus for the study. The results of the study indicate that task orientation and relationship orientation, realiably and validly operationalized through consideration and initiation of structure, are generalizable across cultures. But, the results also reveal significant differences in the perception of these behaviors, suggesting that some aspects of task orientation and relationship orientation are strongly affected by the cultural influences.

This chapter will discuss the theoretical and practical significance of the results along with implications for future research. Before addressing these issues, the study's theoretical and methodological premises are briefly reconsidered.

Theory and Method

The theoretical foundation of this study comes from the significant recurrence throughout the leadership literature of two distinct leadership behaviors, task orientation and relationship orientation. They are widely accepted as the most consistently observed 
and measured behaviors in leadership research (House \& Aditya, 1997). As such, task orientation and relationship orientation are assumed to be generic behaviors, which are universally applied in organizations across cultures, even though they may be uniquely enacted in organizations characterizing each cultural context. Because task orientation and relationship orientation are identifiable, universal leader behaviors, then it seems logical that the enactment (i.e. application) these behaviors would reflect their cultural context. It could be that certain cultures give unique meaning to task orientation and relationship orientation.

Because cultural contexts reflect a collection of many factors and processes, therefore, their effects on psychological constructs are best untangled if the underlying behaviors are defined equivalently (i.e. construct comparability, measurement equivalence) across cultural group (Little, 1997). Even though measurement equivalence of theoretical constructs is a necessary condition for comparing differences between cultural groups, invariance of the construct's measurement level does not exclude the influence of pervasive cultural influences on the construct's behavioral (i.e. latent) level. If cultural influences, such as social desirability, humility, evasiveness or acquiescence (van de Vijver \& Poortinga, 1992; Mullen, 1995; Little, 1997; Cheung \& Rensvold, 2000) are pervasive characteristics of a cultural group, then they are part of the cultural makeup of individuals (Little, 2000). These characteristics (or any other pervasive characteristic of a society) will influence each indicator the same degree and as a result, a construct would, inevitably, reflect such influences. Thus, at the behavioral level, 
pervasive cultural characteristics are not measurement artifacts, but, instead, are reliable and measurable aspects of the individuals' cultural makeup.

These challenges to theory and method lead to two fundamental concerns in this study: 1) the identification of theoretical constructs that can reliably and validly operationalize task orientation and relationship orientation across cultural groups, and 2) the selection of an analytical framework that ensures construct comparability (measurement equivalence) and can detect possible cultural influences on the constructs in a multiple group setting.

To operationalize task orientation and relationship orientation, two wellestablished theoretical leadership constructs, consideration and initiation of structure, are used. These measures are robust and widely accepted in cross-cultural leadership research, discussed earlier in Chapter 4. To establish their generalizability, perceptual data from 2341 managers and engineers are tested in a sequence of hypotheses in multiple group mean and covariance structures (MACS) analyses. Empirical support for each hypothesis tested is based on model fit indices.

Generalizability of Task Orientation and Relationship Orientation

The hypotheses reveal two significant results. First, hypotheses 1 through 4 provide powerful evidence that task orientation and relationship orientation can be 
validly and reliably operationalized through consideration and initiation of structure and, therefore, are generalizable across the 11 cultural groups studied.

Second, hypothesis 5 reveals significant (similarities and) differences in the average perception of task orientation and relationship orientation, suggesting that some aspects of these behaviors are strongly affected by the cultural influences. In contrast to hypotheses 1 and 4, which established the reliability and validity at the measurement level, hypothesis 5 reflects directly interpretable, error-free effects among the constructs at the behavioral (i.e. latent) level. Said differently, task orientation and relationship orientation can be defined equivalently across the 11 cultures studied, yet still demonstrate different relations across cultures. Thus, the similarities and differences in the perceptions of task orientation and relationship orientation found in this study are "true" similarities and differences and may contain information about cultural influences characterizing each cultural context (i.e. groups). The nature of such influences should be examined before results can be meaningfully interpreted across cultures. To examine the effects of cultural characteristics on the constructs, additional hypotheses on the constructs' latent parameters (i.e. variances, covariances or correlations) can be tested across groups. Such construct level tests are illustrated in the next section.

Assessment of Cultural Effects on Task Orientation and Relationship Orientation

Within the MACS analyses framework hypotheses about the nature of possible cultural influences can be meaningfully tested against the true and reliable properties of 
the constructs (i.e. means, variances, and covariances or correlations) in each group. As seen in Table 20, the relative mean difference scores for task orientation and relationship orientation indicate some form of consistency in average perceptions of these behaviors in 8 countries, but also indicate significant differences in average perceptions in the 3 of the 11 cultural groups. What the results do not indicate, however, is whether the managers across 8 groups are more homogeneous in their perceptions of task orientation or relationship orientation than are the managers of the other 3 groups. The results also do not indicate whether the observed similarities and differences are related to the managers' associations between the two behaviors (i.e. how managers relate the behaviors to one another). That is, similar associations (correlation) between the two constructs between two groups suggest that managers in both groups associate task orientation and relationship orientation to the same degree (i.e. strength of the relationship between the two behaviors). Hypotheses on the variances and correlations (i.e. covariances) of the constructs across groups can help answer some of these questions.

Variability (i.e. standard deviation) of a construct reflects the similarity in a group's responses. For example, the lower the variability of task orientation is within a group, the more homogeneous (i.e. identical, uniform) the perceptions (responses) of task orientation are within a group. Thus, if two groups indicate similar variability on task orientation, the lower the variability is, the more homogeneous both groups are in their perception of this behavior. As seen in Figures 16 and 22 (p. 156 and 162), the variability of task orientation (i.e. initiation of structure) is similar in China and Korea 
( 0.49 and 0.50 , respectively), suggesting that the Chinese and Korean managers are equally homogeneous in their perception of task orientation. On the other hand, as seen in Figure 18 (p. 158), the variability of task orientation is much lower in Taiwan (0.26) than is the variability in China and Korea, suggesting that the Taiwanese managers are more homogeneous in their responses to task orientation than are the Chinese and Korean managers. Hypotheses on the variability of constructs are useful, because they can help detect differential socialization practices (e.g. gender effects) across cultural groups (Little, 1997).

Correlations or covariances between constructs reflect the independence of the two constructs (see methodology section). If the covariances (i.e. associations) between task orientation (initiation of structure) and relationship orientation (consideration) are similar between two groups, then the individual-difference associations between these two behaviors are quite robust to cultural or social influences, even if their variances (i.e. standard deviations of the constructs) differ (Little, 1997). As seen in Figures 11, 13, 15, 19,21 , and 23 (p.151, 153, 155, 159, 161 and 163), the covariances (i.e. association) between the two constructs are similar in Taiwan, Japan and Korea $(0.21,0.26$ and 0.22 , respectively) and are similar in Australia, U.S. and Canada (0.31, 0.34 and 0.32), perhaps indicating that within each cultural cluster managers associate task orientation and relationship orientation similarly, even though the variances of the constructs differ. For instance, the variability of task orientation (initiation of structure) of Taiwan $(0.26$, Figure 19, p. 159) is in much lower as compared to the variability of task orientation in Japan and Korean (0.45 and 0.45, Figures 21 and 23, p.161 and 163, respectively). 
These illustrations, albeit hypothetical and brief, demonstrate the effectiveness of construct level hypotheses in cross-cultural research. Construct level hypotheses allow the researcher to discover and examine the influence of cultural characteristics on behaviors within each cultural group studied. Pervasive cultural characteristics must be measured and controlled for when testing for cross-cultural differences in behaviors of interest, so that observed similarities and differences can be meaningfully interpreted.

Theoretical Contributions

The study makes a significant contribution to leadership theory by drawing on the recurrence of two distinct leader behaviors, task orientation and relationship orientation. For almost five decades, these leader behaviors appear throughout the leadership literature as important phenomena in leadership theorizing and research. As such, task orientation and relationship orientation are assumed generic behaviors, which continue to be universally observed and applied in organizations across cultures.

While the logic suggesting universality of these behaviors is compelling, there is little empirical evidence supporting such premise. Yet, a cumulative body of informed and empirically supported research exists, which, to some extent, has identified taskoriented and relationship-oriented behaviors that might be universally applied (e.g. Misumi, 1985; Misumi \& Peterson, 1985; Sinha, 1980; Smith \& Peterson, 1994; Dorfman, 1997). The lack of empirical evidence supporting such an application may be attributed to the broad generalizations about the observed differences in the behaviors, 
which, upon closer examination, are based on complex conceptualization, psychometrically poor measures and inadequate assessment procedures (e.g. Schriesheim \& Kerr, 1977; Strube \& Garcia, 1981; Vecchio, 1983; Peters, et al, 1985; Smith, et al.1989; Ayman and Chemers, 1982; Evans, 1996; Dorfman, 1996; Schriesheim \& Neider, 1996; House \& Aditya, 1997; Drost \& Von Glinow, 1998).

These challenges to theory and method lead to two theoretical contributions of this study. First, it hypothetically develops the universal application of two etic (Berry, 1990) leadership concepts, task orientation and relationship orientation, and empirically confirms the generalizability of their measurement across 11 cultures. Second, the study demonstrates, first empirically and then hypothetically, how to examine the nature of emic (Berry, 1990) or culturally specific influences on the reliable and true properties of the constructs (i.e. means, variances, covariances or correlations) in a quantitative manner (Little, 1997). If specific cultural characteristics exist, they can be measured independently from the behaviors (i.e. construct) of interest; this way, tests of differences across cultures are more likely to reflect real differences or effects.

Methodological Contributions

The study makes a significant contribution in cross-cultural research methodology in two ways. First, the study provides a theoretical understanding of the nature and generalizability (i.e. construct comparability or measurement equivalence) of latent constructs. Second, it demonstrates a practical, albeit powerful, technique, mean and 
covariance structures (MACS) analyses, that ensures construct comparability and can detect possible cultural nuances/influences on the constructs underlying behavior across multiple groups.

MACS analyses provide very robust tests of the validity of construct comparability. The advantages of construct comparability are that the constructs are generalizable to each cultural context, sources of error and bias are minimal, cultural differences have not inconsistently affected the constructs basic measurement characteristics, and the reliable and true properties of the constructs can be assessed for possible cultural influences on the constructs (Little, 1997). The effectiveness of multiple-group MACS analyses is that mean-level information about the indicators of constructs is also analyzed along with the typical variance-covariance information of standard structural equation modeling techniques (Little, 2000).

Cross-cultural studies typically hypothesize cultural differences in individual perceptions and attitudes on the basis of scale scores. Because the observed variables reflect both common and specific sources of variance, as a result, cultural influences may affect both sources at the construct level (Mulaik, 1972). If cultural influences affect the specific variance components of particular indictors of a construct (e.g. an item is perceived differently or poorly translated in one group), nonequivalence of the construct would result (Little, 1997; Mullen, 1995). Construct comparability (i.e. measurement equivalence) holds if cultural characteristics have influenced only the common variance components of a construct's indicators and not the specific variance components of a 
construct's indicators (Little, 1997, p. 214). However, construct comparability (i.e. measurement equivalence) does not exclude a consistent or uniform construct-level bias. If, for instance, acquiescence, social desirability, humility or evasiveness (van de Vijver \& Poortinga, 1992; Mullen, 1995; Little, 1997; Cheung \& Rensvold, 2000) are pervasive characteristics of a cultural group, these characteristics (or any other pervasive characteristic) will influence each indicator to the same degree and a construct would, inevitably, reflect such influences (Little, 2000). At the behavioral level, pervasive cultural characteristics are not measurement artifacts, but instead, are reliable and measurable aspects of the individuals' cultural makeup (Little, 1997, p. 215). Consequently, constructs can be defined equivalently, yet still demonstrate different relations across cultural groups (Little, 1997), which the results of this study revealed.

The MACS analyses framework is particularly useful for multiple group comparisons of (latent) constructs in cross-cultural research, because it simultaneously validates (test) hypothesized factor structures across (cultural) groups and can test the nature of (systematic) cultural influences (i.e. similarities or differences) on many aspects of the constructs (i.e. means, variances, covariances, correlations) (Little, 1997).

In sum, the advantages of the MACS analyses framework are threefold: (1) it is theoretically parsimonious, (b) it is empirically parsimonious, (3) and it provides a theoretical basis by which quantitative cross-group comparisons can be executed (Little, 1997). The MACS analyses framework allows researchers to establish construct comparability, to detect possible (systematic) cross-cultural influences on the constructs, 
and, subsequently, to hypothesize cross-cultural differences on leader behavior independent of pervasive cultural influences.

\section{Limitations}

The limitations of the study relate to instrumentation and sampling.

The measures of leader behaviors are obtained from leader descriptions derived from perceptual measures on a single-survey instrument. However, evidence exists for the validity of behavioral measures when responses are elicited for specific behaviors (Gioia \& Sims, 1985; Yukl \& Van Fleet, 1992). The scale for initiation of structure was slightly modified (i.e. dropping item 6 ("my immediate supervisor stresses high standards of performance for group or unit"), which is noted in the methodology chapter. As seen in Table 5, this modification does not affect the psychometric property of the scale.

The samples vary in terms of breadth and depth of industries surveyed in each country, which may account for some variation in the distributions of the data. The samples vary in size, although most samples are adequate for this study (i.e. $n=144-$ $\mathrm{n}=430$ ). The samples for Canada, Taiwan and the Gulf region are relatively small $(n=118, n=118, n=97)$. The samples are comprised of working managers and engineers, with the exception of two samples derived from executive in MBA programs in the U.S. and executive training programs in Latin America. The samples are underrepresented by female managers (total $24 \%$ ), in particular by Japan and Korean female managers ( $8 \%$ 
and $2 \%$, respectively). However, it is difficult to acquire an equal number of male and female managers in cross-cultural studies, as female managers are often in the minority in many of the lesser-developed countries/regions comprising this sample.

The samples are limited to four cultural/economic regions, North America and Australia, Asia and Latin America. The Latin American region is limited to Mexico, Venezuela, Costa Rica, Nicaragua, Salvador and Panama. The Gulf region is limited to the Jordan and Saudi Arabia. Several developed and lesser-developed regions such as Europe and Africa are not included in the samples. It is important that this limitation is considered, because the socio-cultural and economic differences between these regions and those surveyed are great. The difficulties in obtaining data from numerous cultures are known, however, the study's systematic sampling technique (see method section) and its effort to select an array of diverse cultures increases the possibility of detecting real cross-cultural differences if they truly exist.

Implications for Theory and Practice

Given the study's compelling historical and empirical evidence of the generalizabililty of task orientation and relationship orientation and the significance of cultural influences on these behaviors, it can be concluded that cross-cultural leadership research needs to be directed toward the conditions and contexts under which these behaviors prevail. In a recent critique of current leadership theory, theorists (e.g. Byer, 1999; Shamir \& Howell, 1999; Yukl, 1999) fervently called for psychometrically strong 
measures and for the inclusion of context into the discussion of leader behavior effects.

This study answers such a call in demonstrating a powerful theoretical and practical technique that allows researchers to simultaneously validate hypothesized factor structures and to detect possible cultural influences (i.e. defining contextual characteristics) on the true and reliable properties of psychological constructs.

The contextual and psychometric limitations observed in the extant leadership theory are often associated with the emic-etic (Berry, 1990) argument as to whether prominent U.S leadership theories are generalizable to other countries. Perhaps it is time to reconsider the argument and put forward that etic-imposed constructs are generalizable, if emic (Berry, 1990) or culturally specific "proper contexts" are embedded into the discussion of etic-imposed constructs (Drost \& Von Glinow, 1998), which the study demonstrated hypothetically and empirically.

A major problem in cross-cultural research, as was alluded to earlier in the study, is the limited discussion of possible cultural influences (i.e. context) on psychological constructs. Cross-cultural research typically hypothesizes the effects of etic-imposed, universal behaviors, as a result, these behaviors would, inevitably, reflect their cultural context. The influence of cultural characteristics on behavioral constructs is not always evident at the measurement level and may contribute to misleading effects or measurement artifacts. In contrast, at the behavioral level, pervasive cultural characteristics (e.g. evasiveness, humility, gender biases) are not necessarily measurement artifacts, but instead, are reliable and measurable aspects of the individuals' 
cultural makeup. Such characteristics (or any other pervasive contextual element) can be detected and examined, which this study demonstrated, and - if they occur-should be embedded (i.e. controlled for or measured) within theoretical frameworks. From this point of view, emic (Berry, 1990) or culturally specific characteristics of a society can be measured or embedded into hypothesized relationships, so that the effects of leader behaviors can be observed independent of such characteristics. Adding "proper context" (Drost and Von Glinow, 1998) to etic-imposed leadership concepts provides an important step when testing for between-group differences, because they can help explain observed similarities and differences at a more universal (i.e. etic-imposed) level. While emic studies reveal culturally specific leader behaviors, and even though these behaviors are important to consider, they do not allow sufficient generalizability, nor do they encourage researchers to step back and look for similarities within and between cultures (Drost \& Von Glinow, 1998).

It is clear that the leadership phenomenon is complex to conceptualize and difficult to operationalize, particularly from a cross-cultural perspective. Theorists in the U.S. and abroad continue to investigate the effects of culture and situational factors on leader effectiveness in flexible, albeit complex, contingency models (Fiedler, 1967; Evans, 1970; Vroom \& Yetton, 1973; Fiedler \& Chemers, 1974, 1984; House, 1972; House \& Dessler, 1974; House \& Mitchell, 1974; Vroom \& Jago, 1988; Yukl \& Van Fleet, 1992; House, 1996; Chemers, 1993, 1997), which to some extent have identified task orientation and relationship orientation behaviors, yet, it is not clear under which conditions these two leader behaviors prevail. To understand leadership at the universal 
level, the more recent contingency models are too complex to test empirically or apply practically (e.g. Chemers, 1993, 1997). As a result they do not offer much empirical evidence to current leadership theory.

If leadership theory is an effort by theorists to explain the complex (observed) world of leaders, then the leadership phenomenon must to be simplified conceptually. Current theoretical models do not provide enough guidance in the form of universal leader behaviors to help practitioners recognize the underlying behavioral requirements and choices in the many fragmented activities and problems confronting them. A theory of cross-cultural leadership needs universal behaviors and situational elements that are easy to apply. These universal behaviors must provide managers some flexibility (i.e. choice) yet enable them to distinguish between more or less desirable universal behaviors in particular situations across cultures.

In conclusion, the study proposes a unified theory of cross-cultural leadership. A unified theory of cross-cultural theory must begin with task orientation and relationship orientation, because they are universally accepted and observed in organizations and can be reliably and validly operationalized through consideration and initiation of structure. A unified theory of cross-cultural leadership must unify "proper context" (Drost \& Von Glinow, 1998) into a theoretical framework of task orientation and relationship orientation so that the conditions or situations upon which these behaviors are enacted can be tested independent of cultural characteristics defining each context. A unified cross-cultural leadership theory should be tested within a framework (i.e. MACS 
analyses) that ensures construct comparability and can detect cultural influences on taskoriented and relationship-oriented behaviors. Hence, building toward a unified theory of task-orientated and relationship-oriented leader behavior operationalized from this perspective is more likely to reflect real substantive effects and demonstrate strong nomological validity for theoretical relationships that are being tested. 


\section{Table 1. Indicator Questions for Consideration and Initiation of Structure}

\section{Initiation of Structure}

$X_{1} \quad$ Sets specific goals for me to accomplish ( $1=$ not al all, $5=$ to a very great extent)

$\mathrm{X}_{2} \quad$ Emphasizes high standards of performance ( $1=$ not al all, $5=$ to a very great extent)

$\mathrm{X}_{3} \quad$ Stresses the importance of work goals $\quad(1=$ not al all, $5=$ to a very great extent)

$\mathrm{X}_{4} \quad$ Checks everything; individual judgment is not trusted

( $1=$ not al all, $5=$ to a very great extent)

\section{Consideration}

$\mathrm{X}_{5} \quad$ Is friendly and easy to approach

( $1=$ not al all, $5=$ to a very great extent)

$\mathrm{X}_{6} \quad$ Is eager to recognize and reward good performance

(1=not al all, 5=to a very great extent)

$\mathrm{X}_{7} \quad$ Is willing to listen to my problems

( $1=$ not al all, $5=$ to a very great extent)

$\mathrm{X}_{8} \quad$ Treats me with respect

( $1=$ not al all, $5=$ to a very great extent)

$\mathrm{X}_{9} \quad$ When suggestions are made to top management, they receive fair evaluation $\quad(1=$ not al all, $5=$ to a very great extent) 
Table 2. Sequence of Hypotheses Test for Equality of Latent Means

\begin{tabular}{|c|c|c|}
\hline Hypotheses & Symbol & Constraints \\
\hline $\mathrm{H} 1$ & $\mathrm{H}_{\text {form }}$ & $\begin{array}{l}\text { Same factor structure (Figure } 2 \text { ). The same factor structure } \\
\text { holds when the baseline model indicates adequate fit across } \\
\text { groups. }\end{array}$ \\
\hline $\mathrm{H} 2$ & $\mathrm{H}_{\Lambda \mathrm{x}}$ & $\begin{array}{l}\text { Equality of factor loadings (Figure } 3 \text { ). Factor loadings are } \\
\text { constrained to be equal across groups. Factorial invariance } \\
\text { exists if there is no significant difference in fit between } \mathrm{H} 2 \\
\text { and } \mathrm{H} 1 \text { across groups. }\end{array}$ \\
\hline $\mathrm{H} 3$ & $\mathrm{H}_{\Lambda v}$ & $\begin{array}{l}\text { Equality of intercepts. (Figure 4) The same factor form } \\
\text { (H 1) and equality of factor loadings (H 2) are required } \\
\text { before testing restrictions on means and intercepts. Equality } \\
\text { of intercepts exists if there is no significant difference in fit } \\
\text { between } \mathrm{H} 3 \text { and } \mathrm{H} 2 \text { across groups. }\end{array}$ \\
\hline H3 IS & $\mathrm{H}_{\Lambda v}$ & $\begin{array}{l}\text { Equality of intercepts of initiation of structure (IS). } \\
\text { (Figure 5) Intercepts of consideration are free. The same } \\
\text { factor form ( } \mathrm{H} 1 \text { ) and equality of factor loadings ( } \mathrm{H} \text { 2) are } \\
\text { required before testing restrictions on means and intercepts. } \\
\text { Equality of intercepts in IS exists if there is no significant } \\
\text { difference in fit between } \mathrm{H} 4 \text { (IS) and } \mathrm{H} 2 \text { across groups. } \\
\text { No constraints are placed on measurement errors. }\end{array}$ \\
\hline $\mathrm{H} 3 \mathrm{C}$ & $\mathrm{H}_{\Lambda v}$ & $\begin{array}{l}\text { Equality of intercepts of consideration (Figure 6). } \\
\text { Intercepts of initiation of structure are free. The same factor } \\
\text { form (H } 1 \text { ) and equality of factor loadings (H 2) are } \\
\text { required before testing restrictions on means and intercepts. } \\
\text { Equality of intercepts for } \mathrm{C} \text { exists if there is no significant } \\
\text { difference in fit between } \mathrm{H} 4(\mathrm{C}) \text { and } \mathrm{H} 2 \text { across groups. No } \\
\text { constraints on measurement errors. }\end{array}$ \\
\hline$\overline{\mathrm{H} 4}$ & $\mathrm{H}_{\lambda \Theta}$ & $\begin{array}{l}\text { Equality of measurement error variances (Figure 7). The } \\
\text { same factor form, (H 1) and equality of factor loadings } \\
\text { (H 2) are required. Measurement error variances are } \\
\text { constrained to be equal across groups. Equality of } \\
\text { measurement error variances exists if there is no significant } \\
\text { difference in fit between } \mathrm{H} 3 \text { and } \mathrm{H} 2 \text { across groups. }\end{array}$ \\
\hline $\mathrm{H} 5$ & $\mathrm{H}_{\Lambda v \mathrm{~K}}$ & $\begin{array}{l}\text { Equality of latent means. The same factor form } \mathrm{H} 1 \text {, } \\
\text { equality of factor loadings } \mathrm{H} 2 \text {, and the invariance of } \\
\text { intercepts } \mathrm{H} 4 \text { are required before testing restrictions on } \\
\text { means. The equality of means for exists if there is no } \\
\text { significant difference between } \mathrm{H} 5 \text { and } \mathrm{H} 4 \text {. }\end{array}$ \\
\hline
\end{tabular}




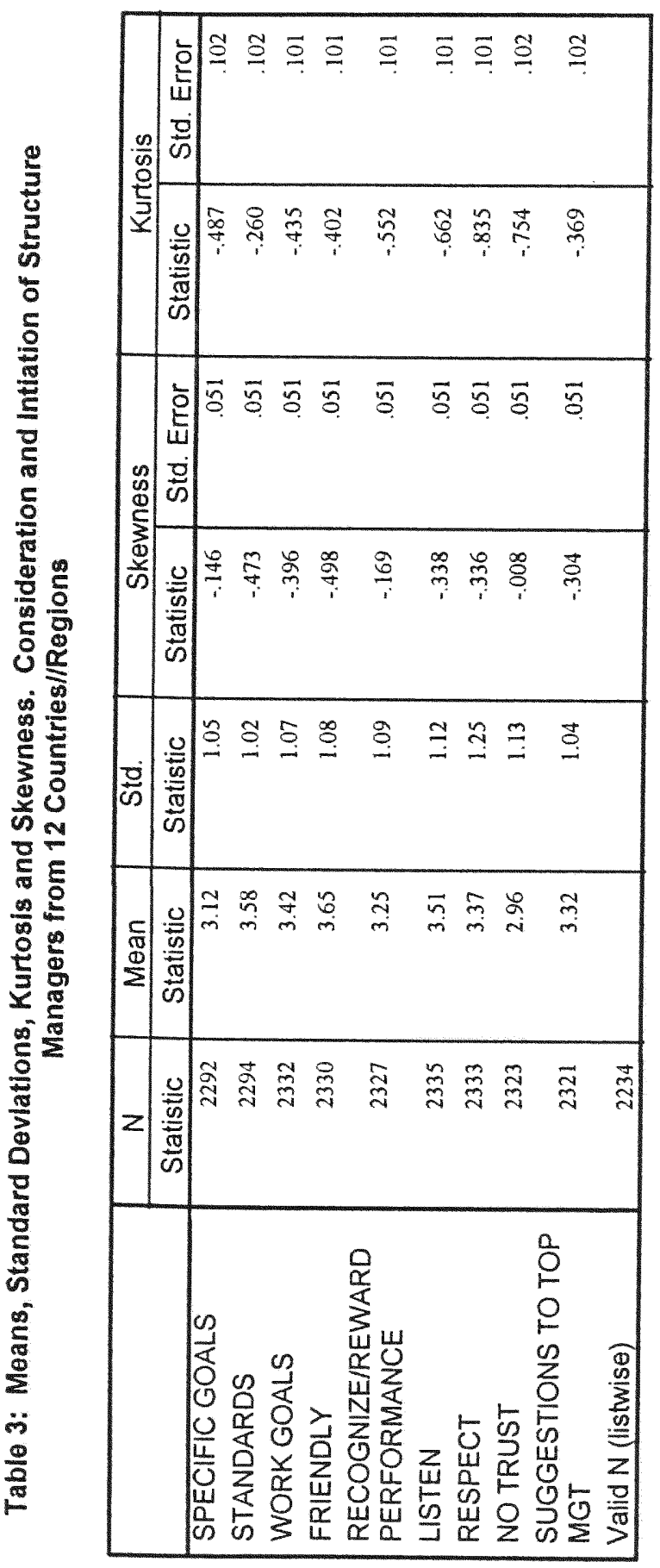




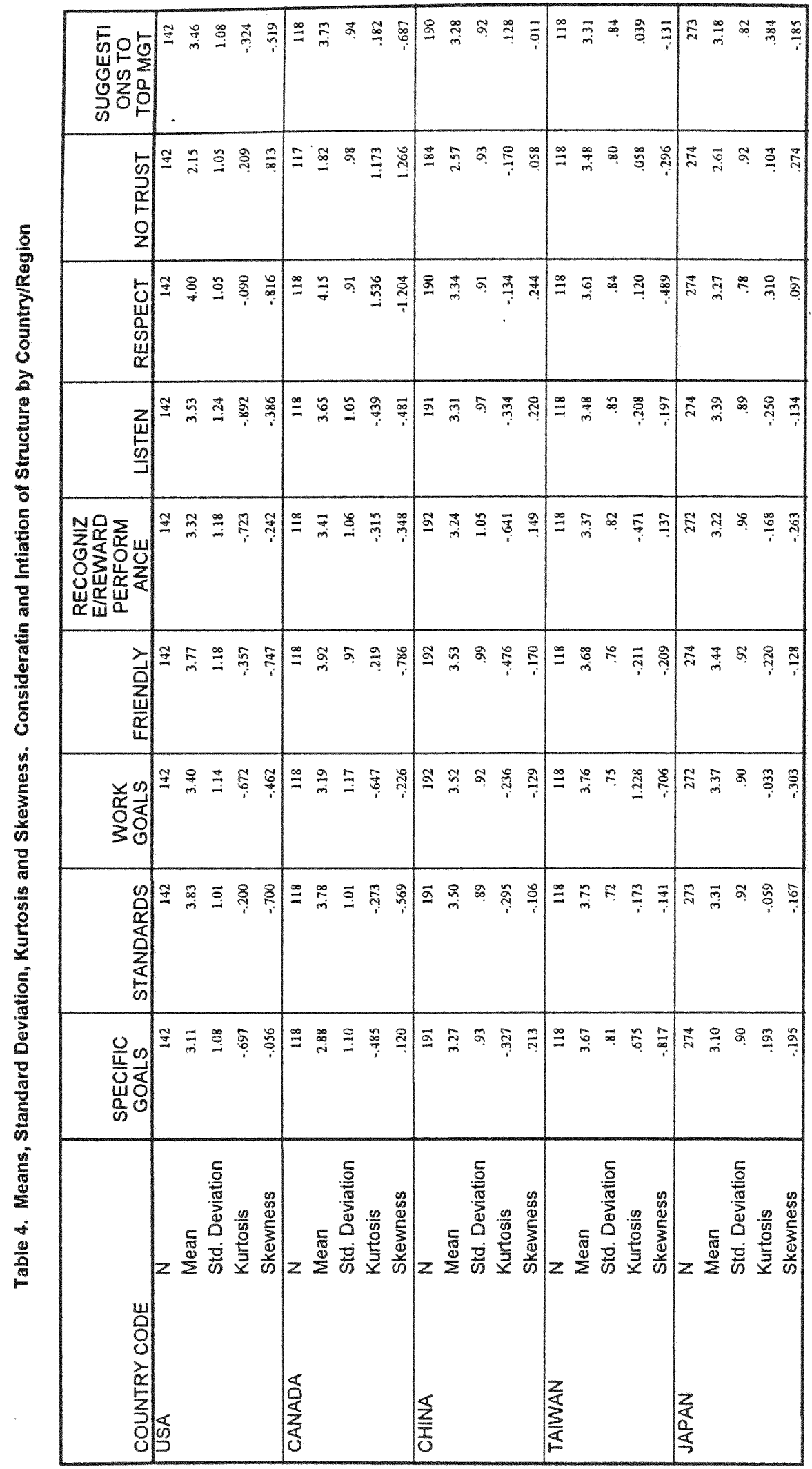




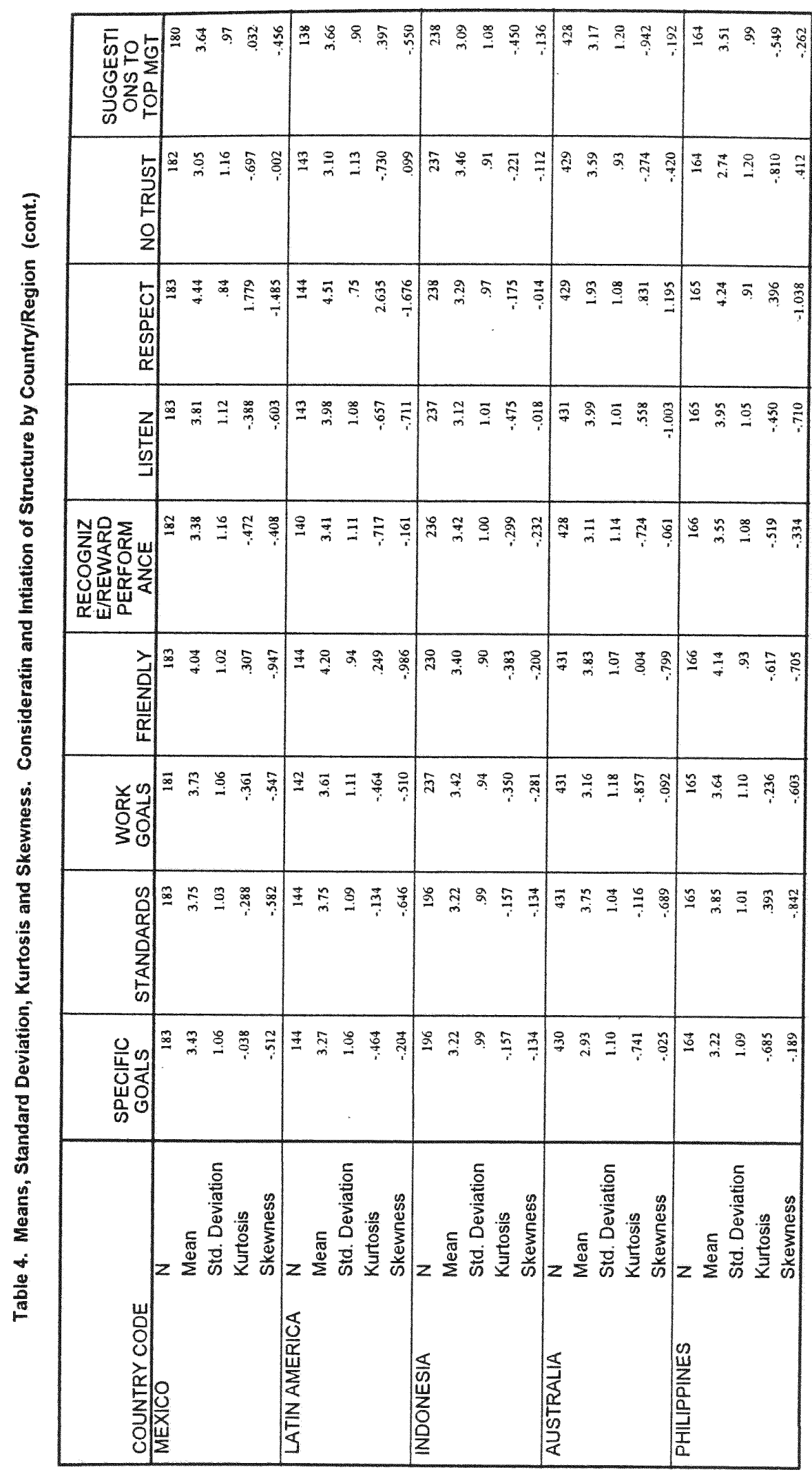




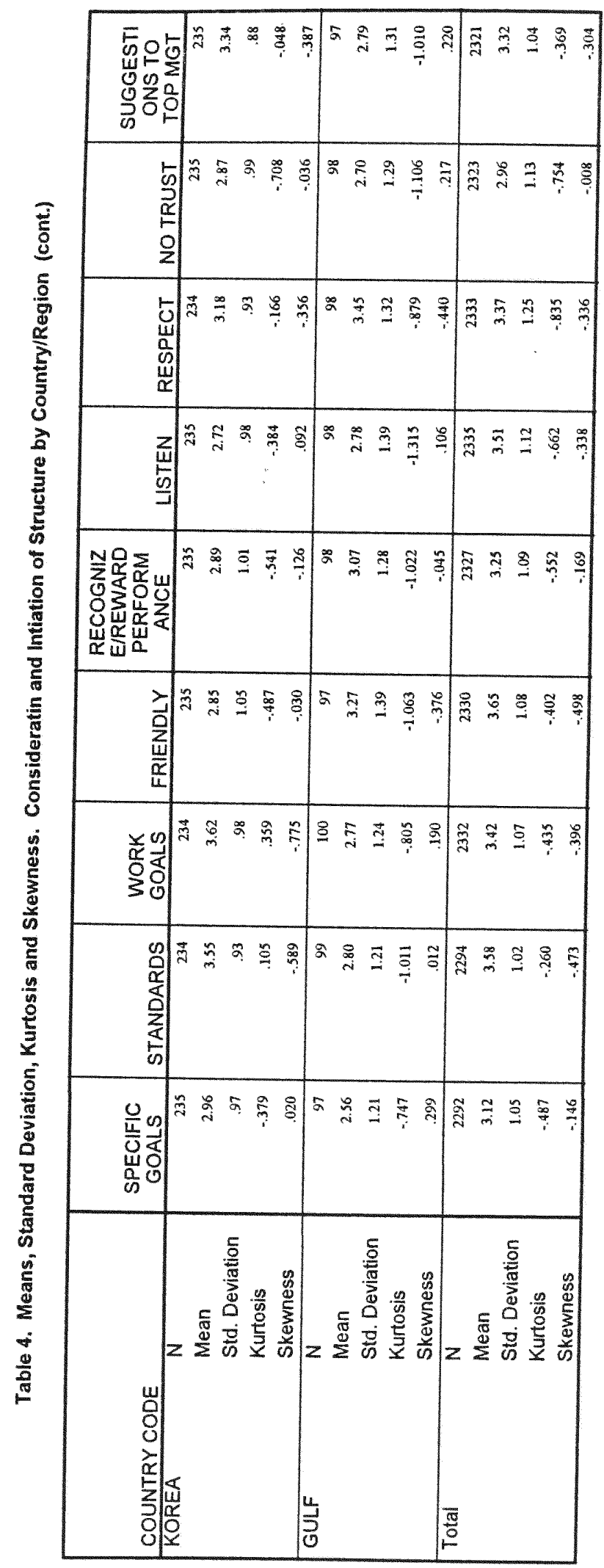


Table 5. Reliabilities for Consideration and Initiation of Structure by Country/Region

\begin{tabular}{|c|c|c|c|c|c|}
\hline COUNTRY & C\&IS & $\mathrm{C}(5)$ & IS (5) & IS (4) & IS (3) \\
\hline $\begin{array}{l}\text { AUSTRALI } \\
\text { A }(n=421)\end{array}$ & .79 & .50 & .74 & .74 & .80 \\
\hline $\begin{array}{l}\text { CANADA } \\
(\mathrm{n}=117)\end{array}$ & .79 & .84 & N/A & .59 & .87 \\
\hline $\begin{array}{l}\text { U.S.A. } \\
(\mathrm{n}=142)\end{array}$ & .81 & .90 & $.72^{*}$ & .64 & .77 \\
\hline $\begin{array}{l}\text { CHINA } \\
(n=182)\end{array}$ & .91 & .91 & .80 & .75 & .86 \\
\hline $\begin{array}{l}\text { TAIWAN } \\
(\mathrm{n}=118)\end{array}$ & .86 & .87 & N/A & .73 & .75 \\
\hline $\begin{array}{l}\text { JAPAN } \\
(\mathrm{n}=272)\end{array}$ & .82 & .82 & N/A & .74 & .83 \\
\hline $\begin{array}{l}\text { KOREA } \\
(\mathrm{n}=232)\end{array}$ & .84 & .84 & .77 & .71 & .79 \\
\hline $\begin{array}{l}\text { GULF } \\
(\mathrm{n}=94)\end{array}$ & .81 & .76 & N/A & .75 & .76 \\
\hline $\begin{array}{l}\text { INDONESI } \\
\mathrm{A}(\mathrm{n}=182)\end{array}$ & .78 & .76 & N/A & .43 & .28 \\
\hline $\begin{array}{l}\text { PHILIPPIN } \\
\text { ES }(n=162)\end{array}$ & .89 & .88 & .81 & .72 & .86 \\
\hline $\begin{array}{l}\text { LATIN } \\
\text { AMERICA } \\
(n=141)\end{array}$ & .78 & .83 & N/A & .72 & .78 \\
\hline $\begin{array}{l}\text { MEXICO } \\
(\mathrm{n}=177)\end{array}$ & .85 & .80 & N/.A & .75 & .85 \\
\hline $\begin{array}{l}\text { TOTAL } \\
\text { SAMPLE }\end{array}$ & .81 & .78 & N/A & .70 & .80 \\
\hline
\end{tabular}

C\&IS = one factor scale reliability

$\mathrm{C}=5$ item scale

IS $=5$ item scale

IS (4) $=4$ item scale

IS (3) $=3$ item scale

*5 item scale based on $n=94$ 


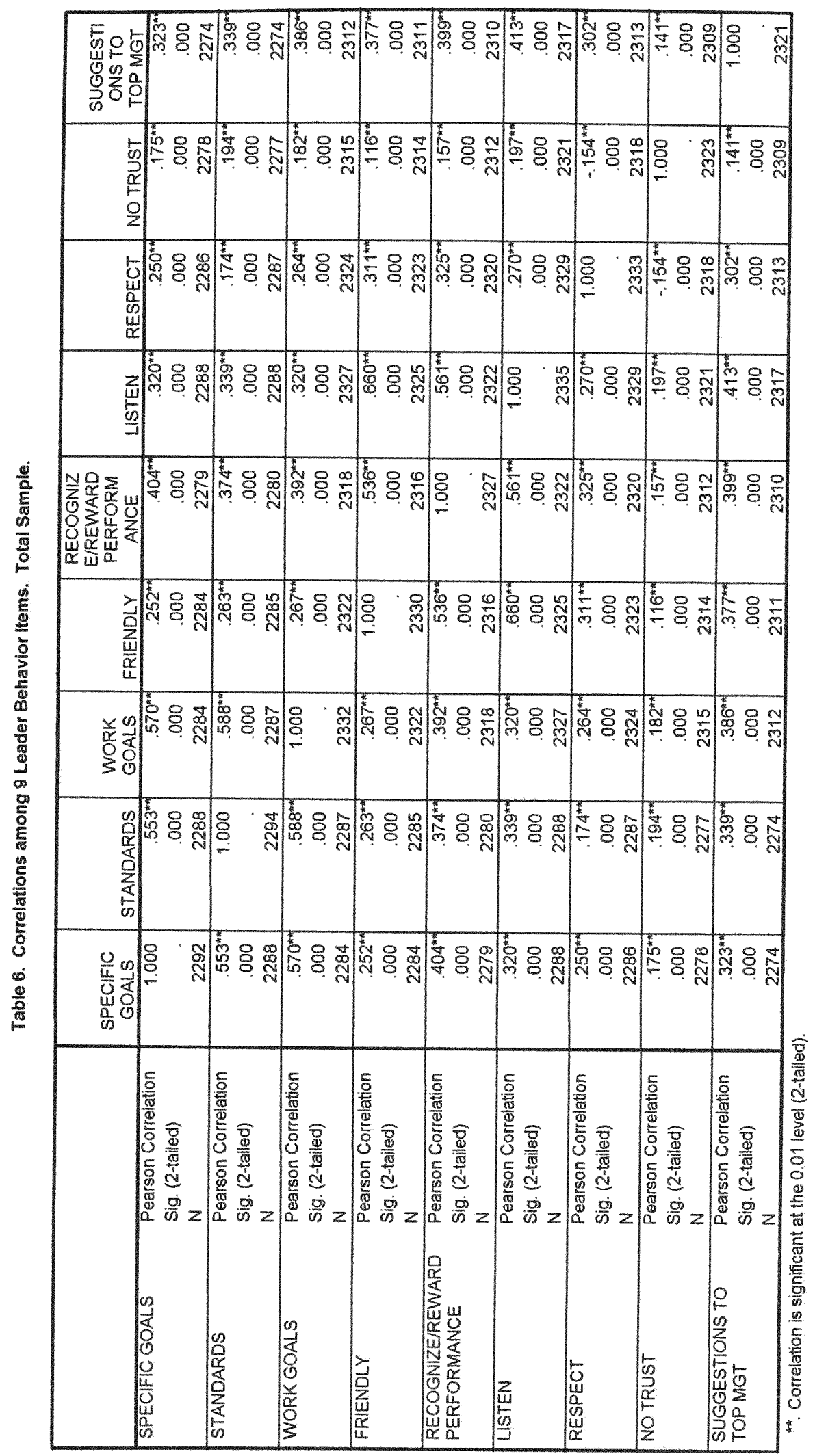




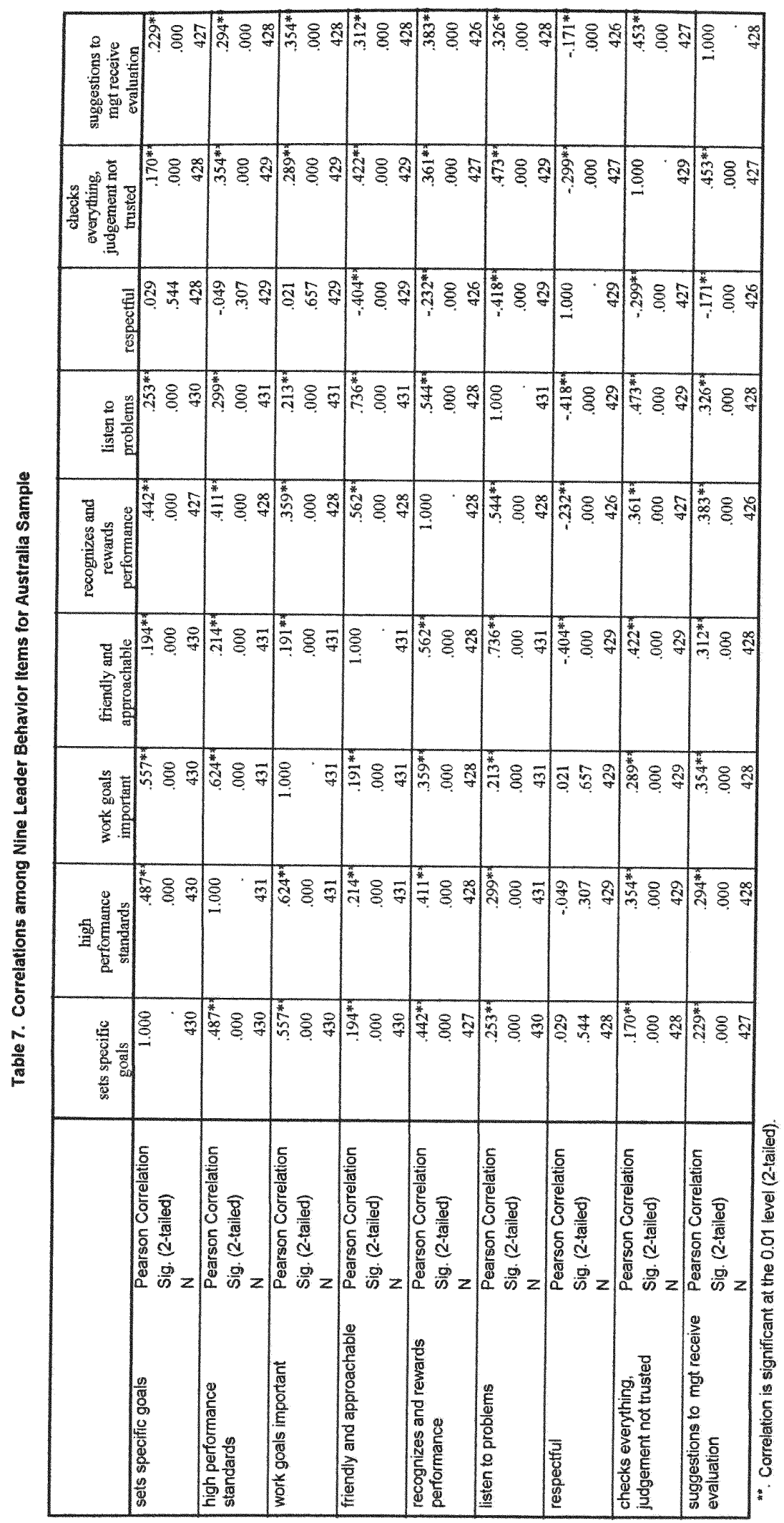




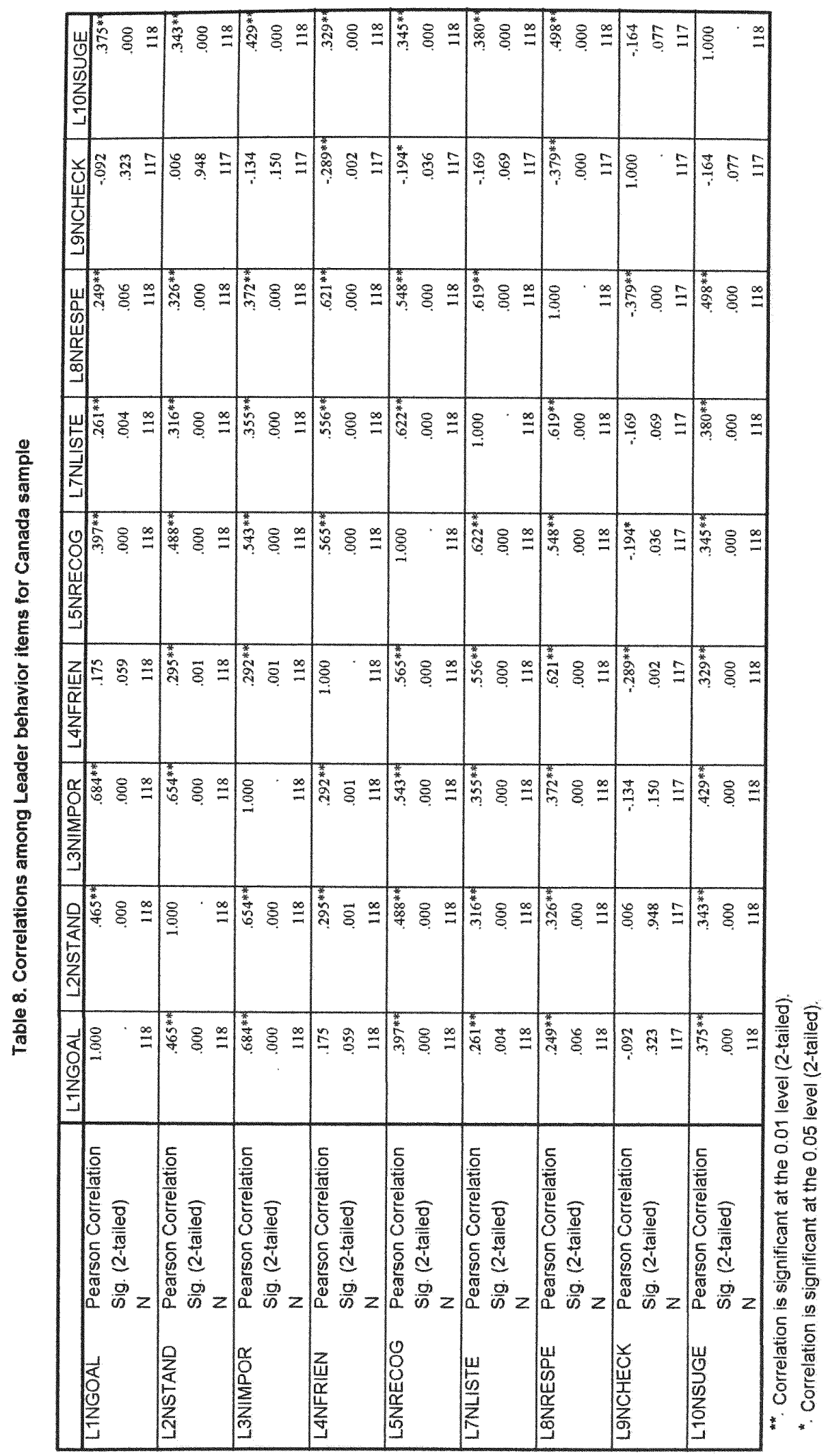




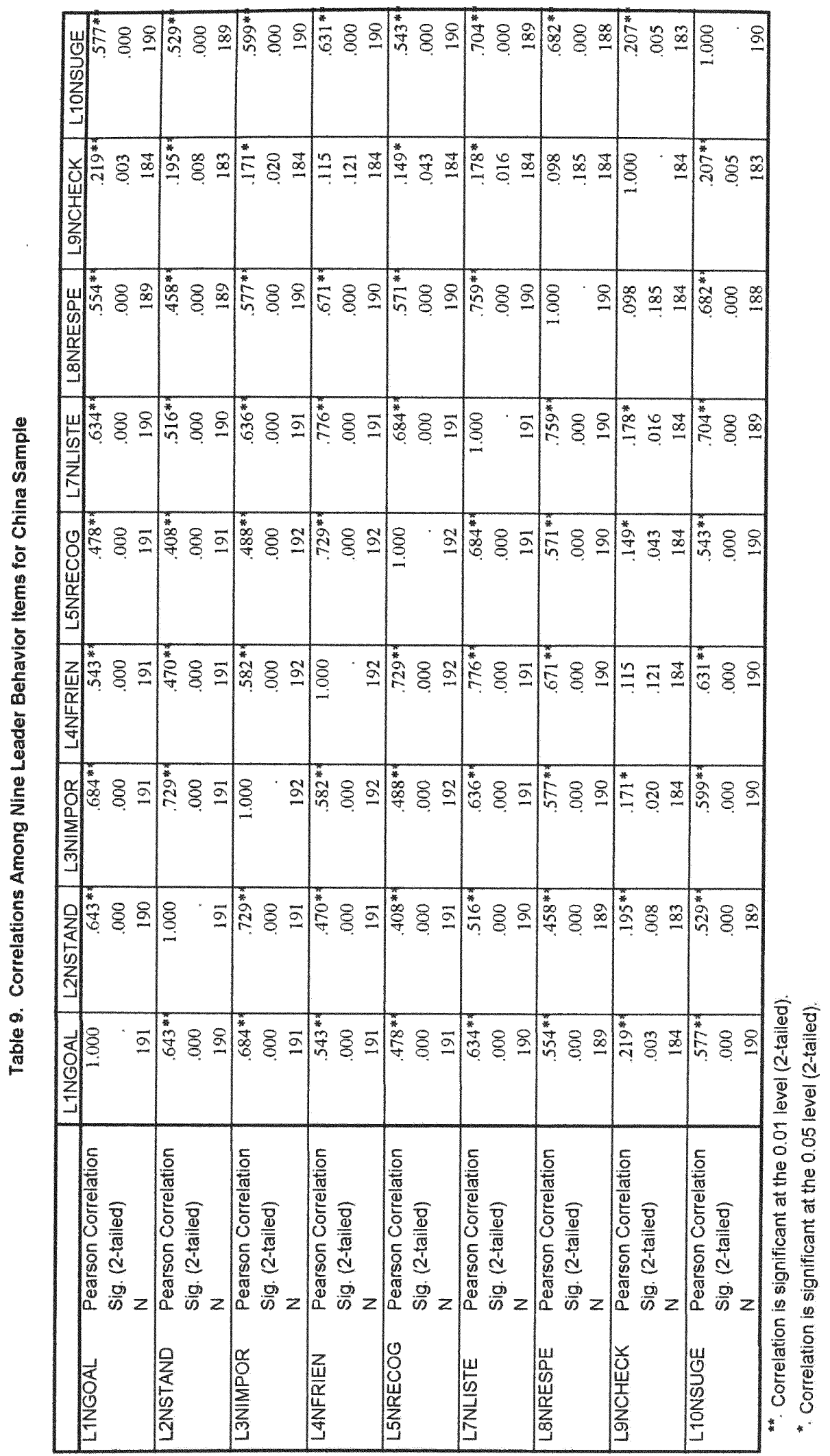




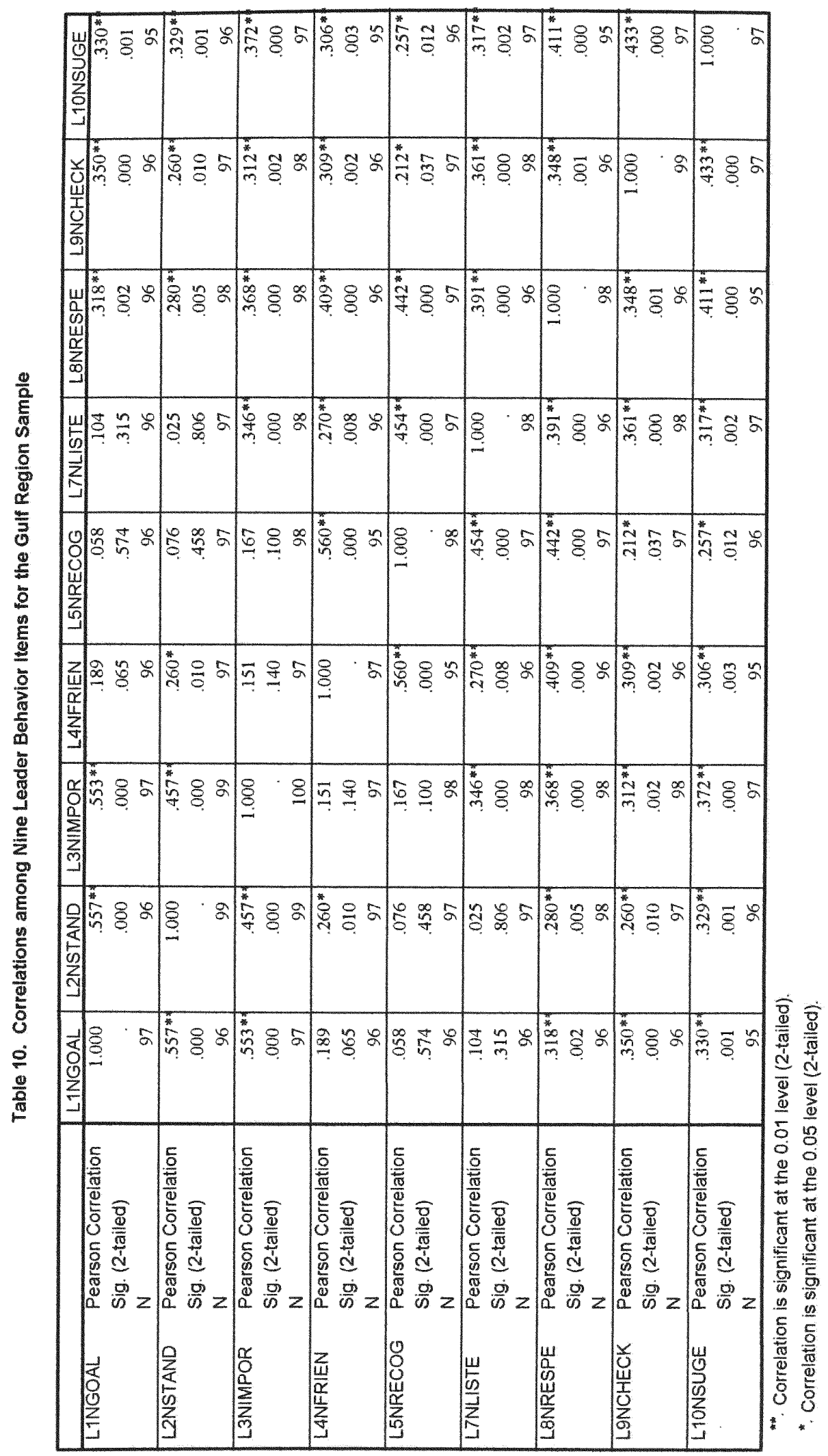




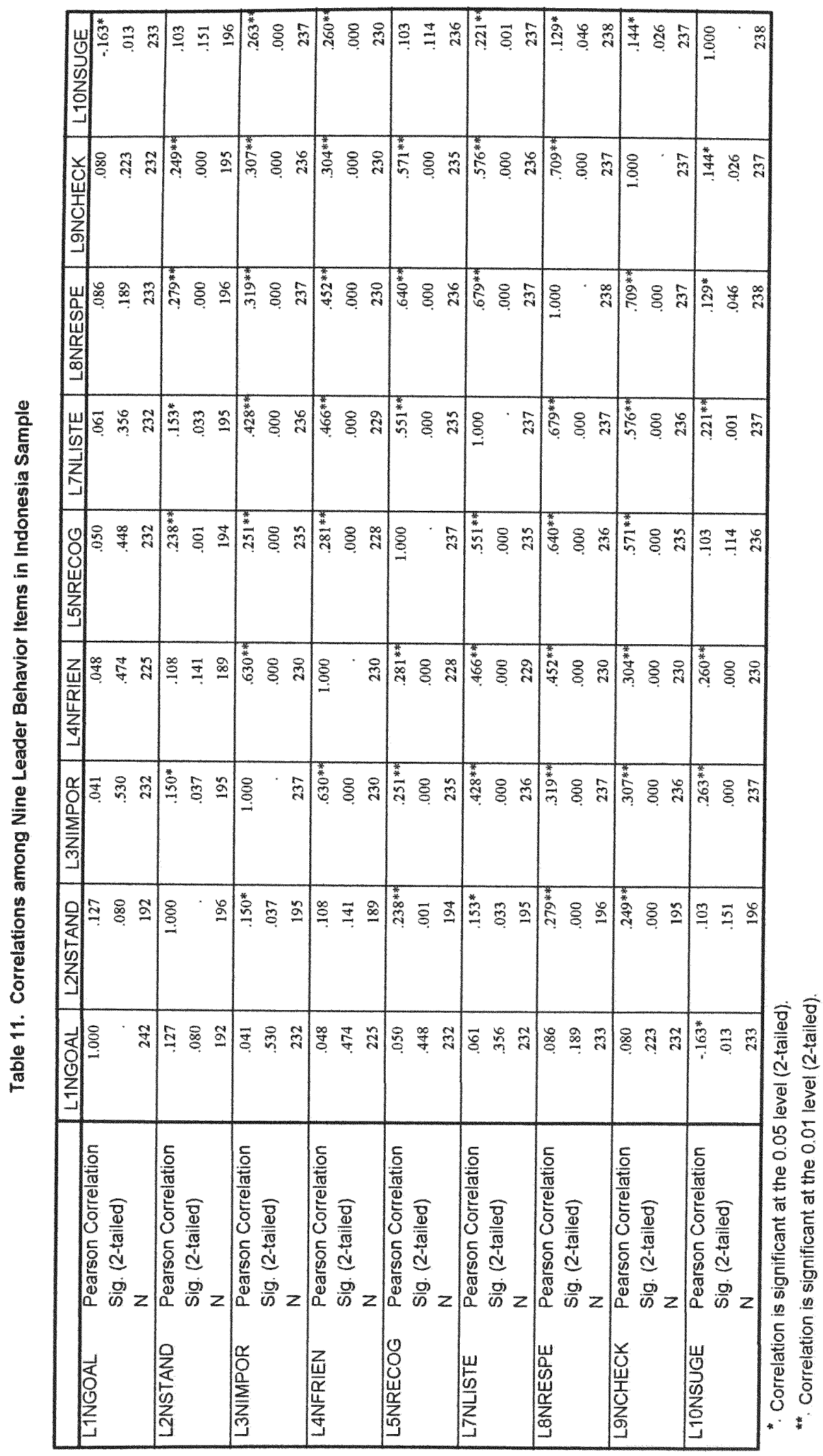




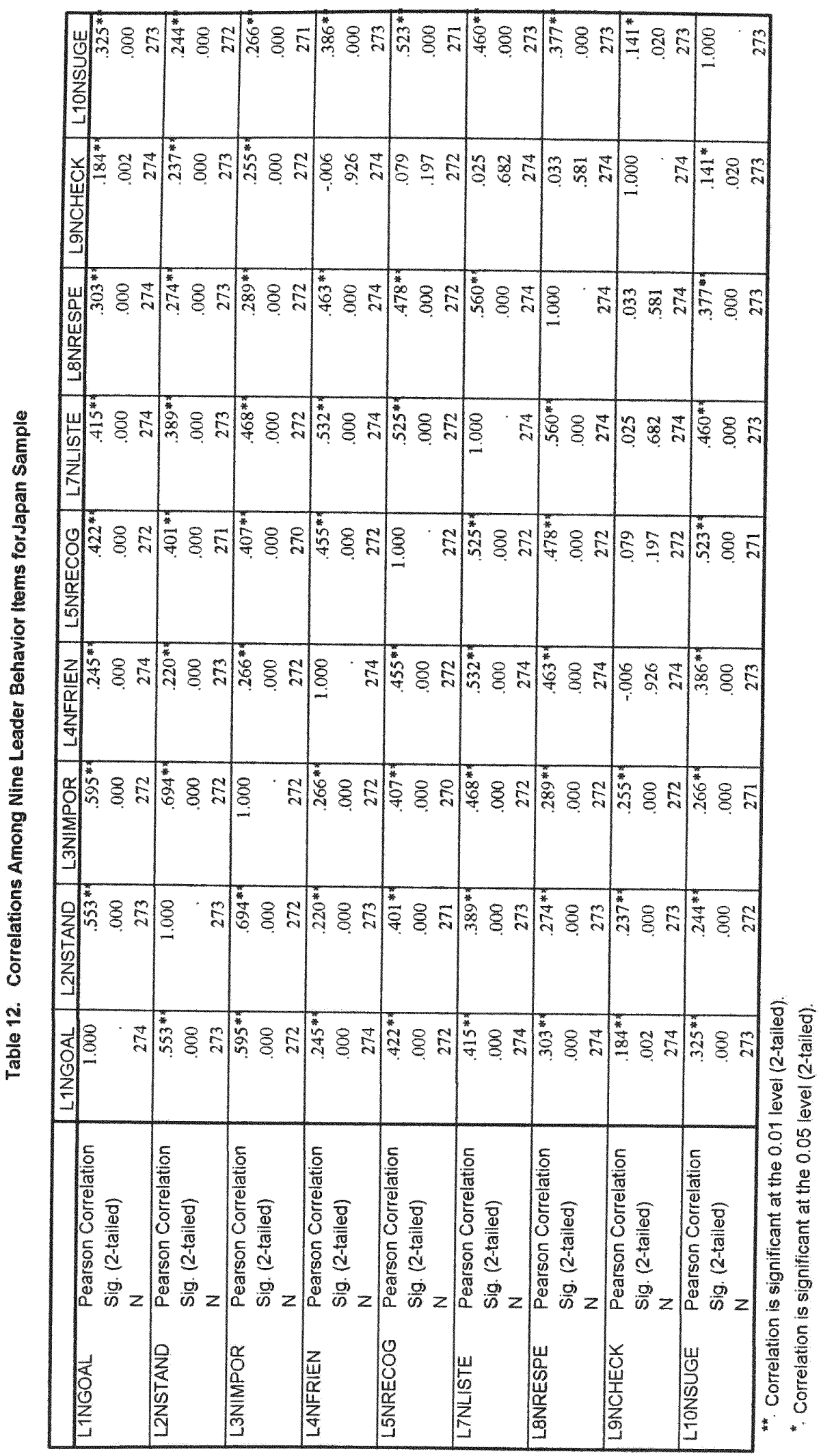




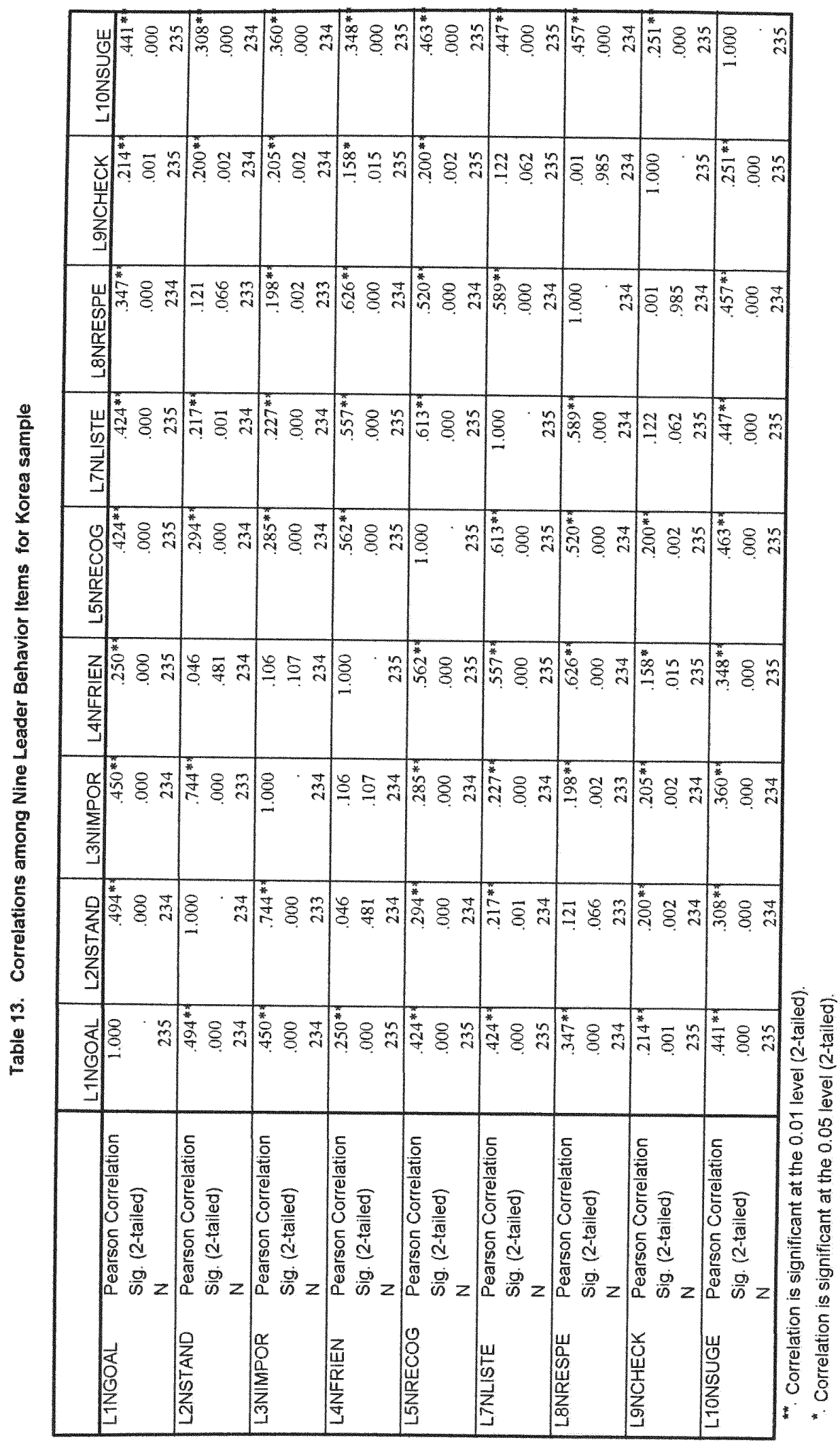




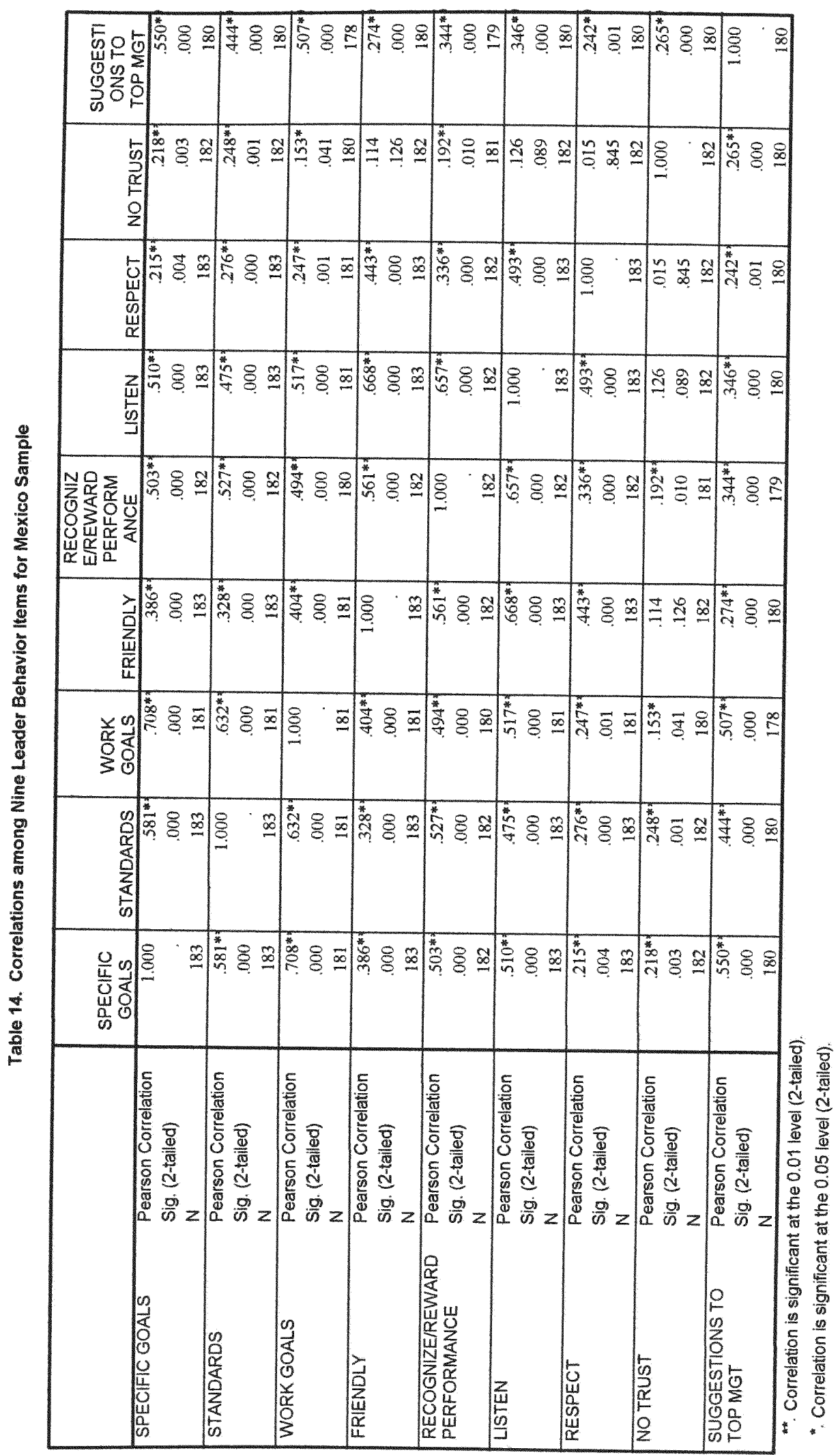




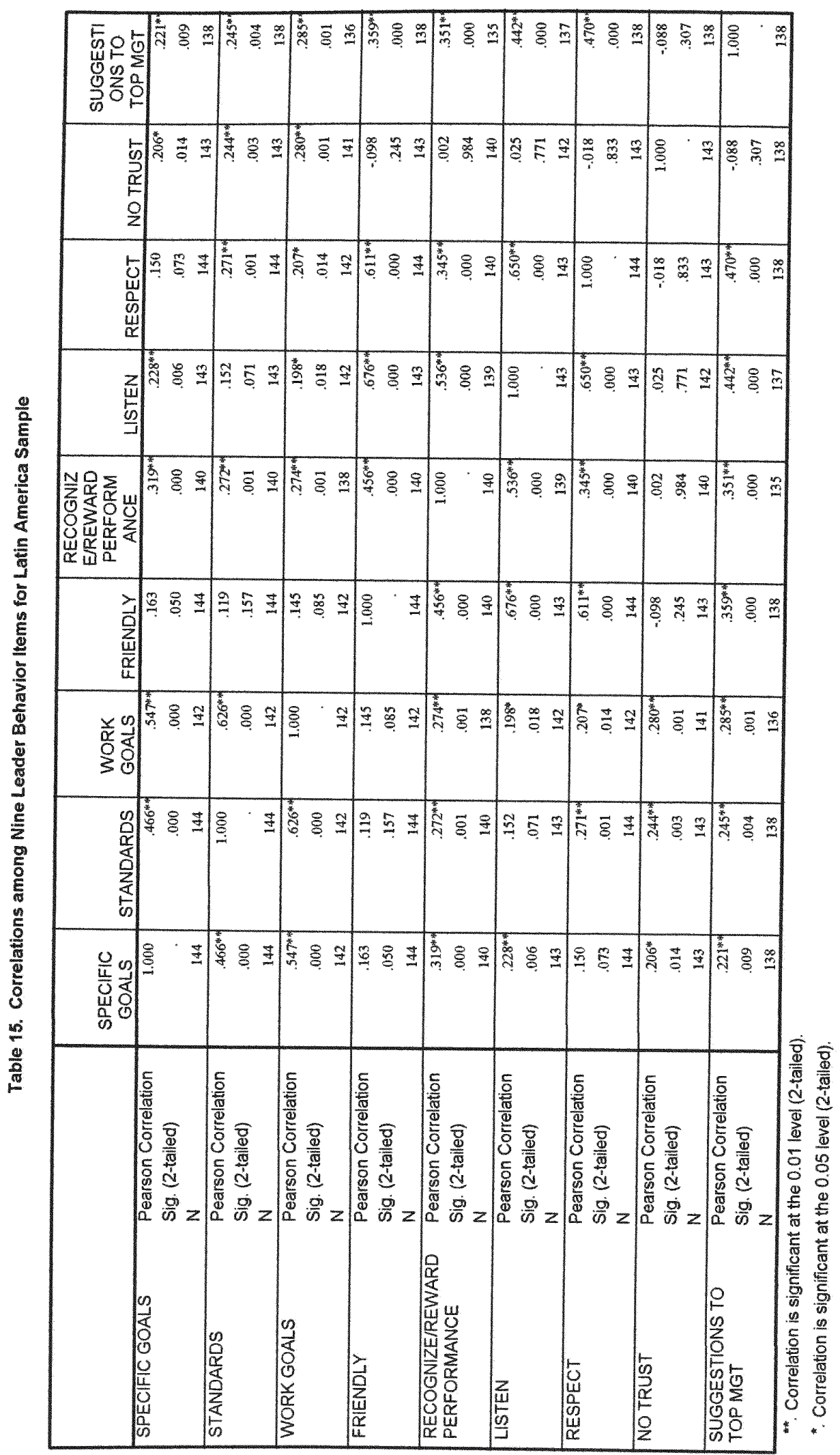




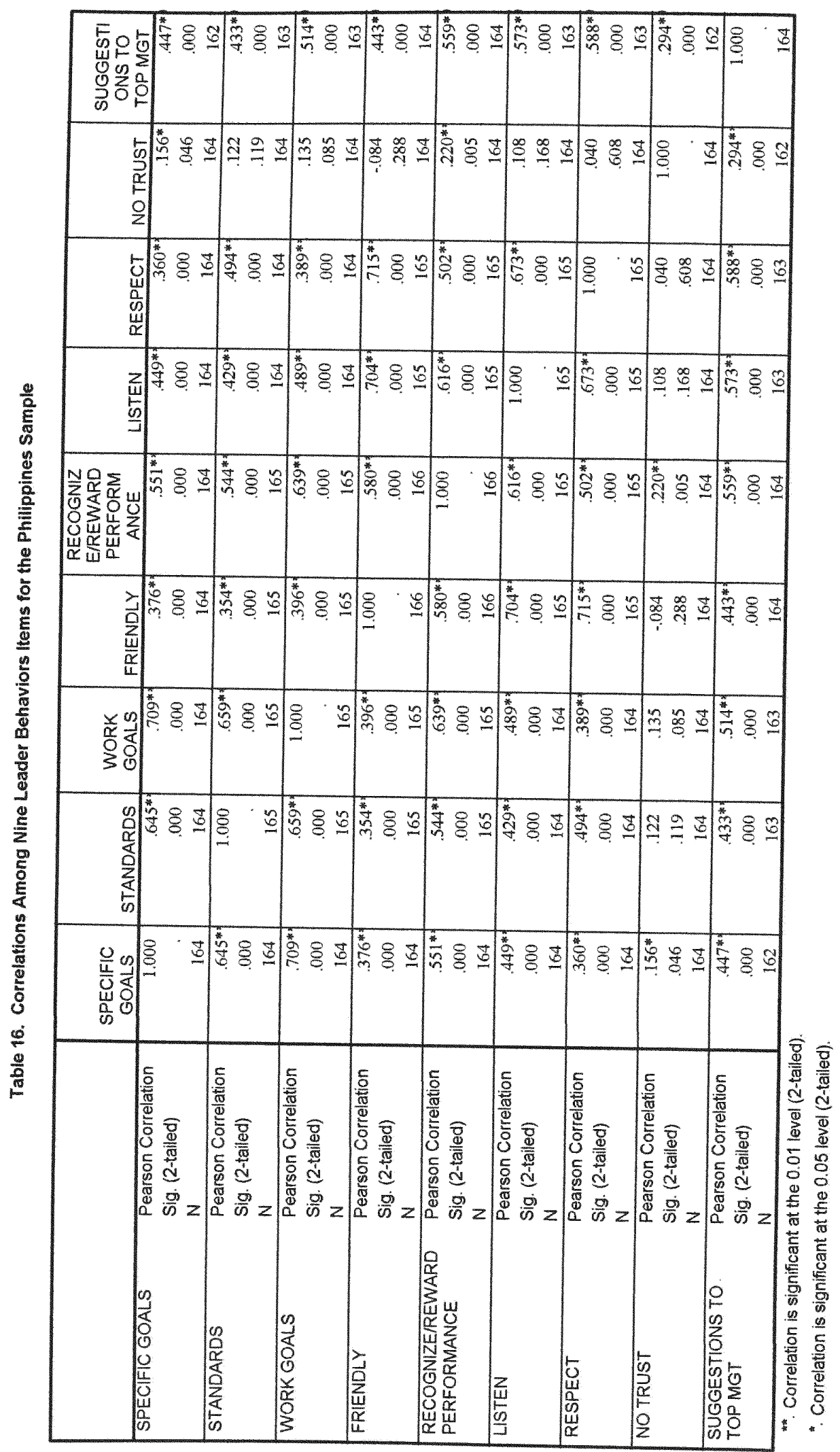




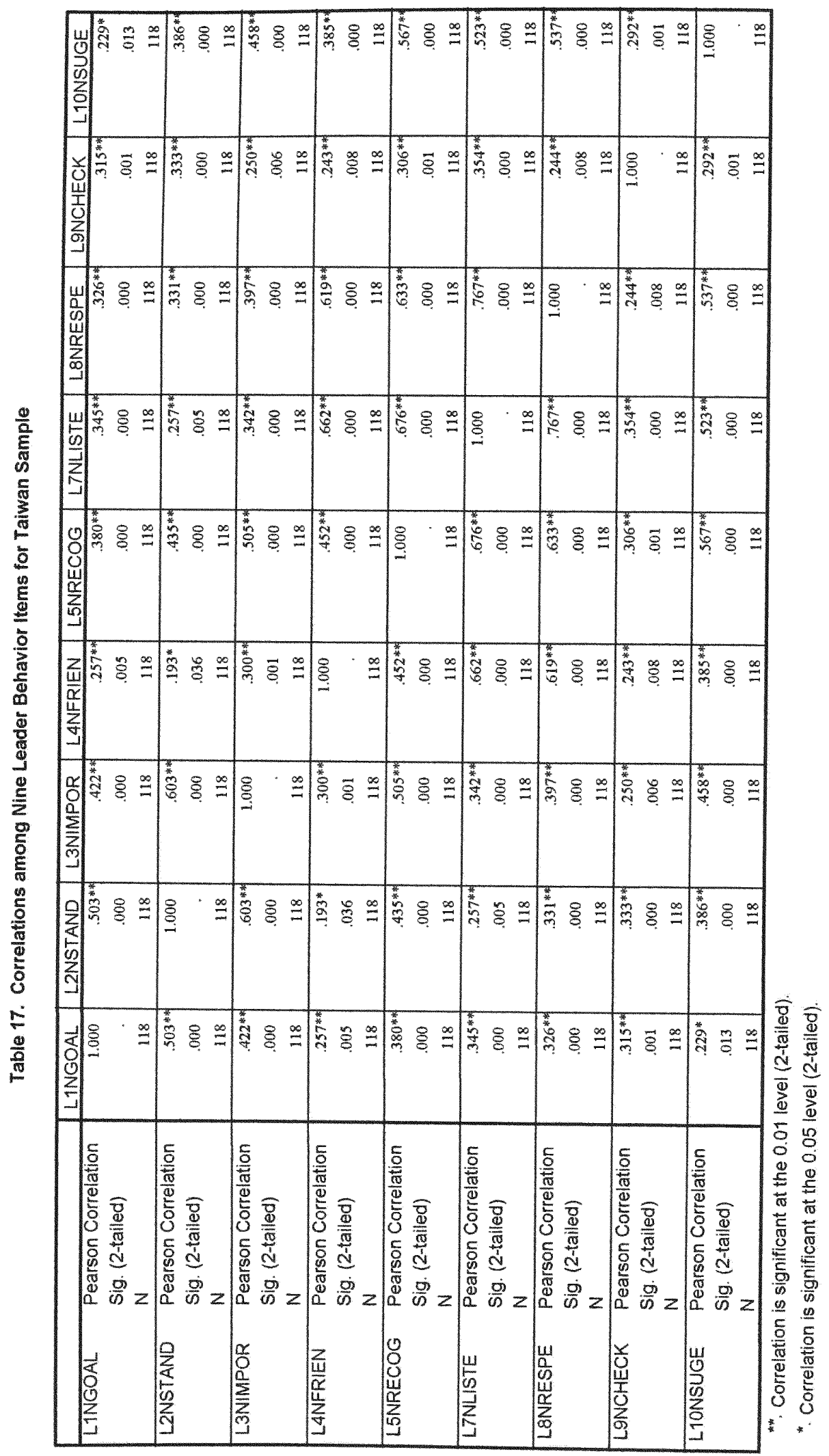







Table 19. Results: Equivalence of Latent Means across 11 countries/regions Multiple-Group Mean and Covariance Structures Analysis

\begin{tabular}{|l|l|l|l|l|l|l|l|l|l|l|}
\hline H\# & $\chi^{2 *}$ & Df & $\Delta \chi^{2 *}$ & $\Delta$ df & CFI & TLI & $\Delta$ TLI & RSMEA & $\Delta$ RSMEA & Pclose \\
\hline H1 & 1117.814 & 286 & $-\cdots$ & $-\cdots$ & .987 & .978 & & .034 & $-\cdots$ & 1.000 \\
\hline H2 & 1472.794 & 356 & 668.661 & 70 & .979 & .971 & -.006 & .038 & .004 & 1.000 \\
\hline H3 & 3691.049 & 446 & 2403.784 & 90 & .940 & .934 & -.037 & .057 & .020 & .000 \\
\hline H3IS & 2283.314 & 396 & 902.939 & 40 & .965 & .957 & -.014 & .047 & .009 & .991 \\
\hline H3C & 2872.814 & 406 & 1487.625 & 50 & .955 & .945 & -.026 & .053 & .015 & .001 \\
\hline H4 & 2633.688 & 446 & 1553.478 & 90 & .960 & .955 & -.016 & .048 & .010 & .968 \\
\hline H5 & 3691.049 & 446 & 1170.945 & 00 & .947 & .939 & .005 & .056 & .001 & .000 \\
\hline
\end{tabular}

${ }^{*} \mathrm{p}=.0000$

NOTE: CFI=comparative fit index; TLI=Tucker \&Lewis Index; RMSEA=root mean square error of approximation. Results: Intercepts for initiation of structure are equivalent across groups meeting both statistical and modeling criteria. Intercepts for consideration are equivalent across groups meeting modeling criteria, CFI and TLI, only. Means for both consideration and initiation of structure are equivalent across groups meeting modeling criteria, CFI and TLI, only. Relative mean differences for consideration and initiation of structure are reported in Table 5.

1. Calculation of differences: $\mathrm{H}(i)=\chi^{2}$, TLI, or RMSEA

$\Delta \mathrm{H} 2=\mathrm{H} 2-\mathrm{H} 1$

$\Delta \mathrm{H} 3=\mathrm{H} 3-\mathrm{H} 2$

$\Delta \mathrm{H} 4=\mathrm{H} 4-\mathrm{H} 2$

$\triangle \mathrm{H} 5=\mathrm{H} 5-\mathrm{H} 3$

2. $\mathrm{H} 3 \mathrm{C}$ and $\mathrm{H} 5$ indicate nonequivalence due to:

$\triangle R M S E A$ significant (Browne \& Cudeck, 1993)

Pclose $<.05$ (Browne \& Cudeck, 1993) 
Table 20. Estimated Relative Mean Difference Scores for Consideration and Initiation of Structure across 11 Countries/Regions

\begin{tabular}{|c|c|c|c|c|}
\hline Country & \multicolumn{2}{|c|}{$\begin{array}{lc}\text { Difference in } & \text { Critical Ratio } \\
\text { Estimated Mean (critical } t \text { value } \\
\text { Scores } & p<0.05 \text { ) }\end{array}$} & $\begin{array}{l}\text { Difference in } \\
\text { Estimated Mea } \\
\text { Scores } \\
\text { Consideration }\end{array}$ & $\begin{array}{l}\text { ical Ratio } \\
\text { cal } t \text { value } \\
<0.05) \\
t \text { value }\end{array}$ \\
\hline Australia & -0.070 & -0.848 & 0.030 & 0.312 \\
\hline United States* & $\begin{array}{ll}0.000 & \text { Fix } \\
& \text { Sco }\end{array}$ & $\begin{array}{l}0.000 \\
0^{*}\end{array}$ & $\cdots$ & --.-- \\
\hline Canada & -0.181 & -1.659 & 0.174 & 1.494 \\
\hline China & 0.018 & 0.201 & -0.309 & $-2.847^{* *}$ \\
\hline Taiwan & 0.287 & $3.220^{* *}$ & -0.134 & -1.214 \\
\hline Japan & -0.130 & -1.562 & -0.327 & $-3.337^{* *}$ \\
\hline Korea & 0.012 & 0.141 & -0.720 & $-6.947 * *$ \\
\hline Philippines & 0.156 & 1.576 & 0.333 & $3.012^{* *}$ \\
\hline Gulf & -0.635 & $-5.298 * *$ & -0.547 & $-4.022 * *$ \\
\hline Latin America & 0.131 & 1.297 & 0.435 & $3.951^{* *}$ \\
\hline Mexico & 0.227 & $2.391^{* *}$ & 0.255 & $2.354^{* *}$ \\
\hline
\end{tabular}

${ }^{*}$ By specifying zero value for means for the U.S. sample, the model is identified.

** Significant at $p<0.05, t$ value $>1.96$

NOTE: The relative mean difference scores on the constructs, consideration and initiation of structure, are an estimation of the unit difference between the U.S. fixed mean of zero and the respective country/region means on consideration and initiation of structure. The critical ratio (CR) provides a significance test based on the critical value for a standard normally distributed random variable. The question asked here is whether the country or region's mean differences are significantly different from zero, $t$ value larger than 1.96 are significant at $p<.05$ level. Significant differences were indicated in Taiwan, Gulf regions and Mexico for initiation of structure, and in most countries/regions for consideration with the exception of Australia, Canada and Taiwan. 


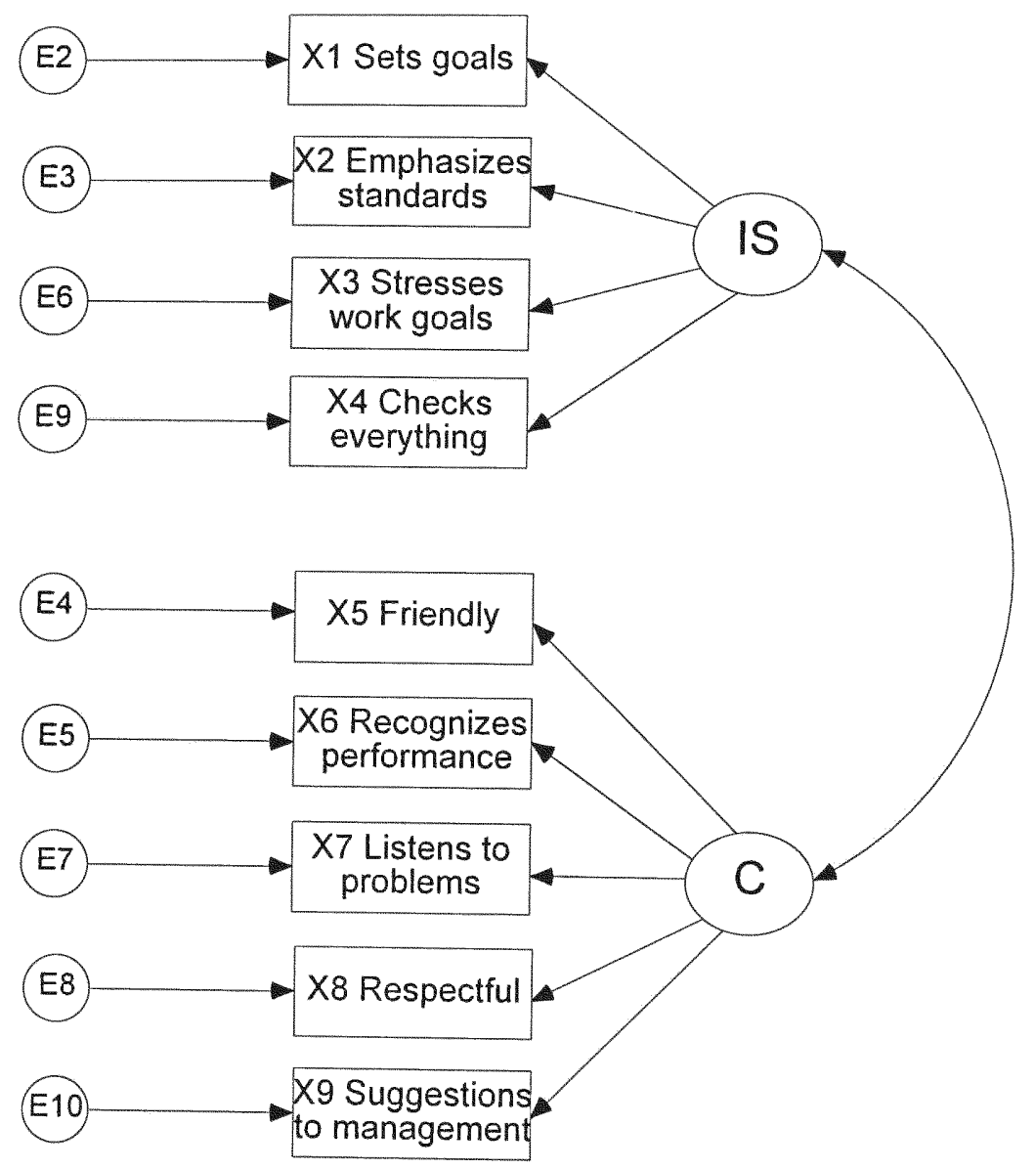

FIGURE 1

TWO FACTOR MODEL

CONSIDERATION AND INITIATION OF STRUCTURE 


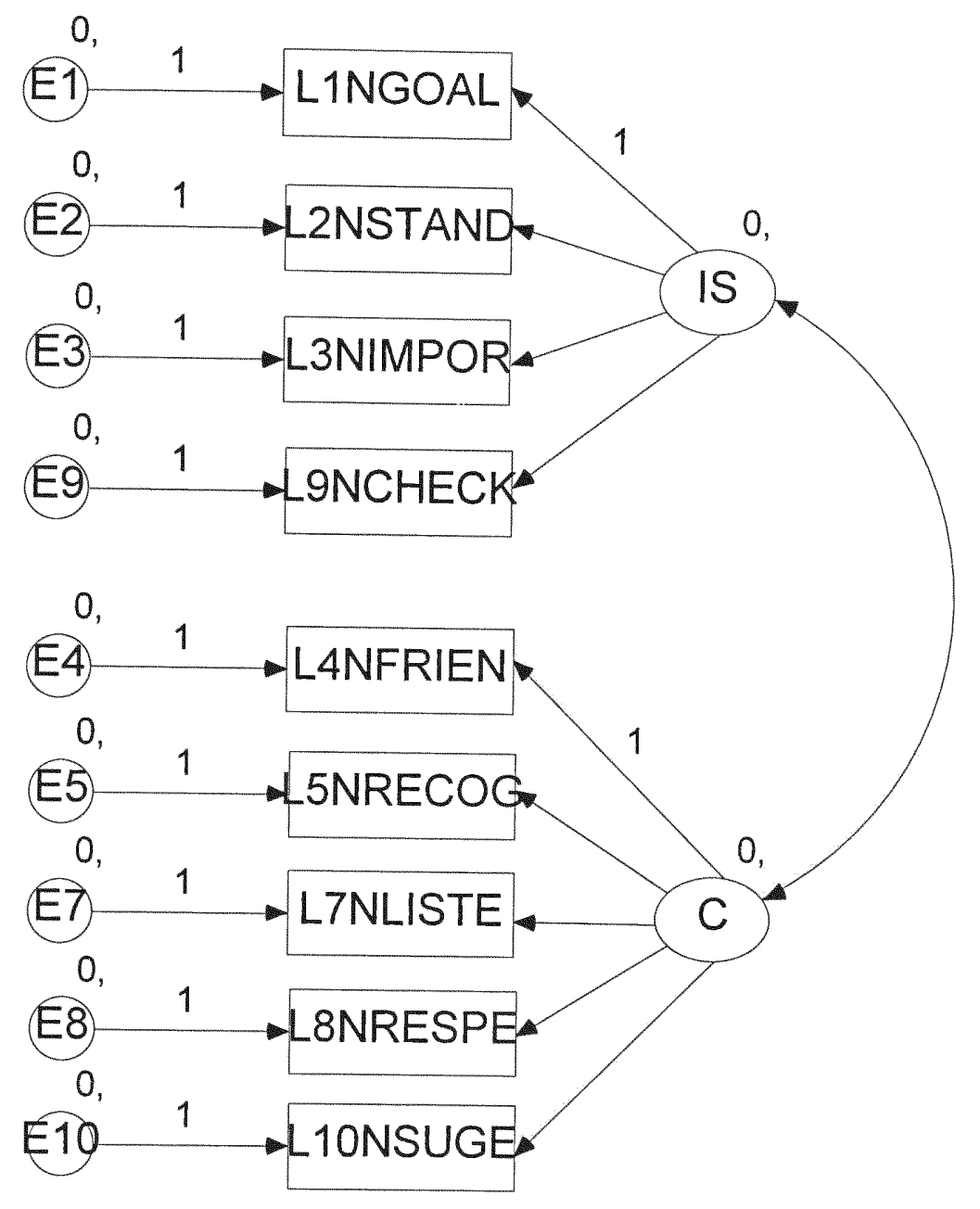

FIGURE 2: HYPOTHESIS 1

SAME FACTOR STRUCTURE

CONSIDERATION AND INITIATION OF STRUCTURE

MEASUREMENT MODEL 


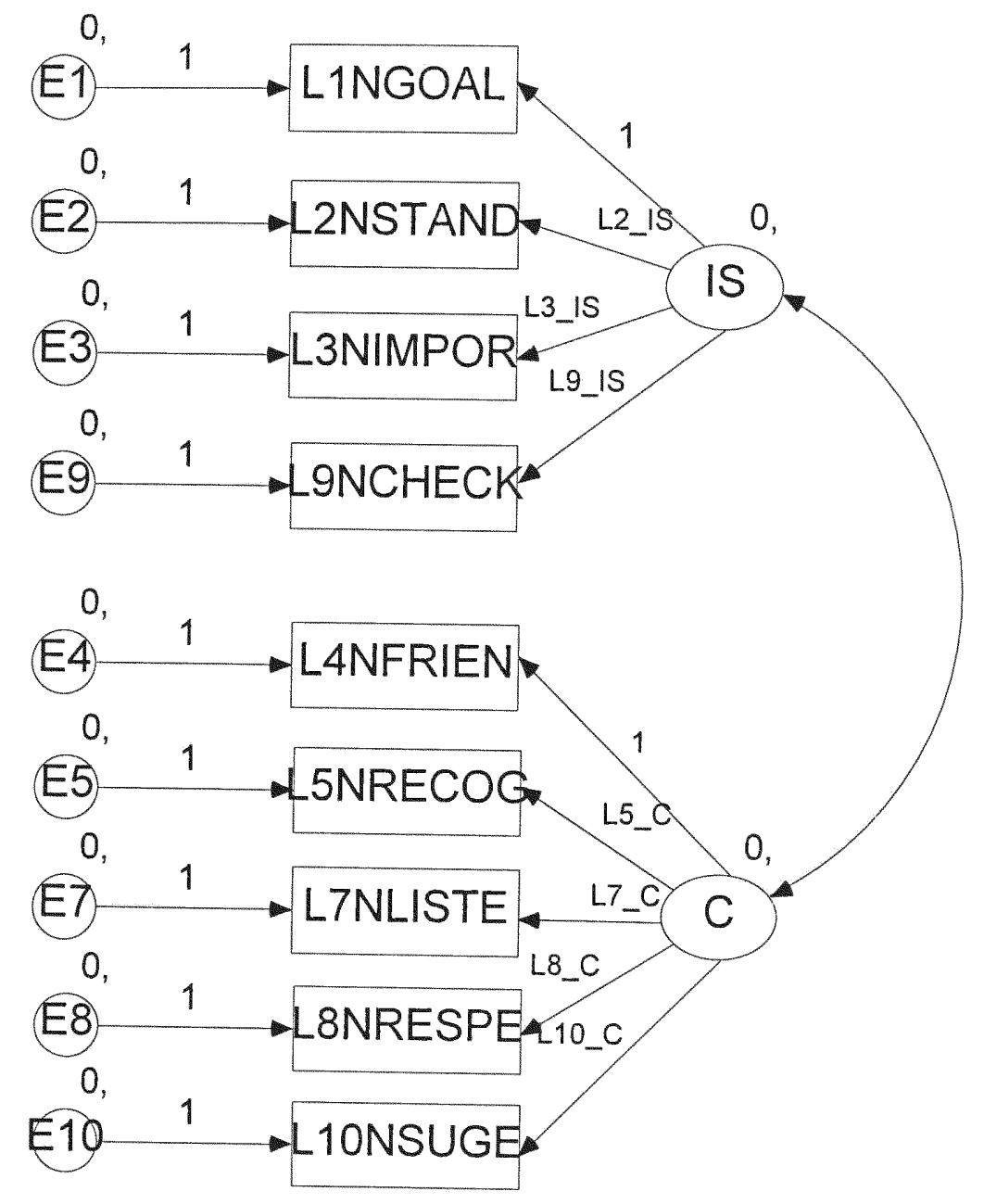

FIGURE 3: HYPOTHESIS 2

SAME FACTOR LOADINGS

CONSIDERATION AND INITIATION OF STRUCTURE MEASUREMENT MODEL 


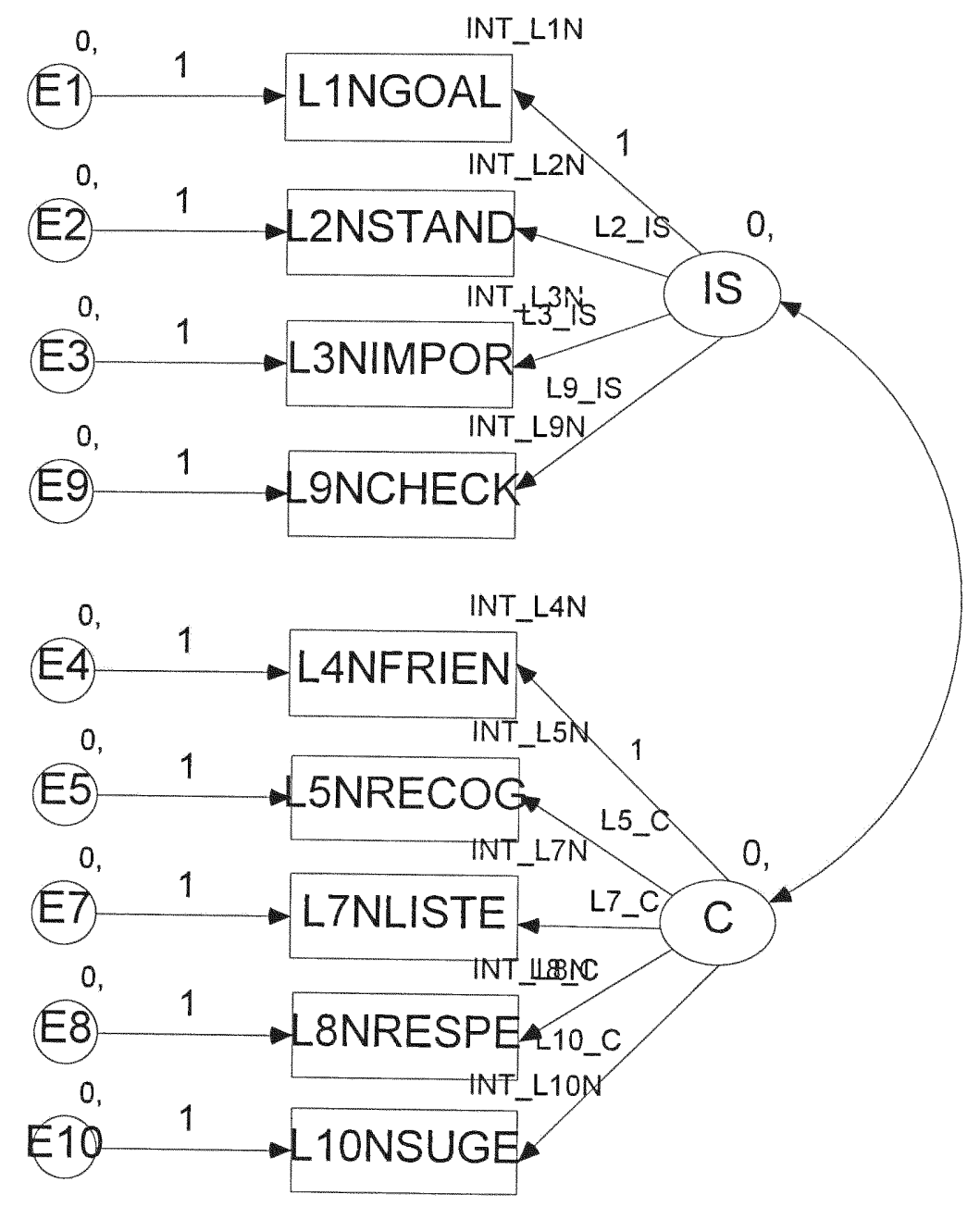

FIGURE 4: HYPOTHESIS 3

EQUALITY OF INTERCEPTS

MEASUREMENT MODEL 


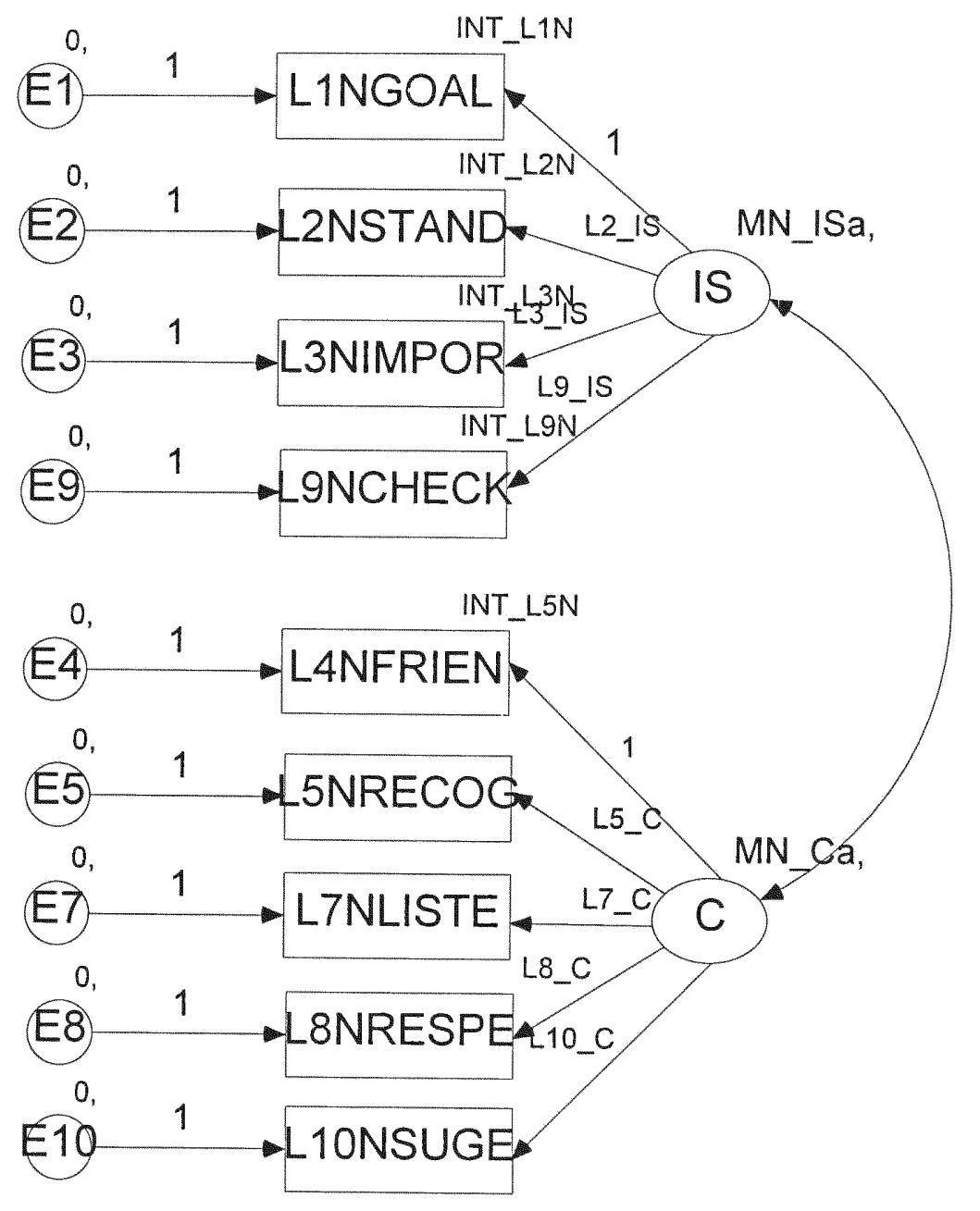

FIGURE 5: HYPOTHESIS 3 (IS)

EQUALITY OF INTERCEPTS

INITIATION OF STRUCTURE

MEASUREMENT MODEL 


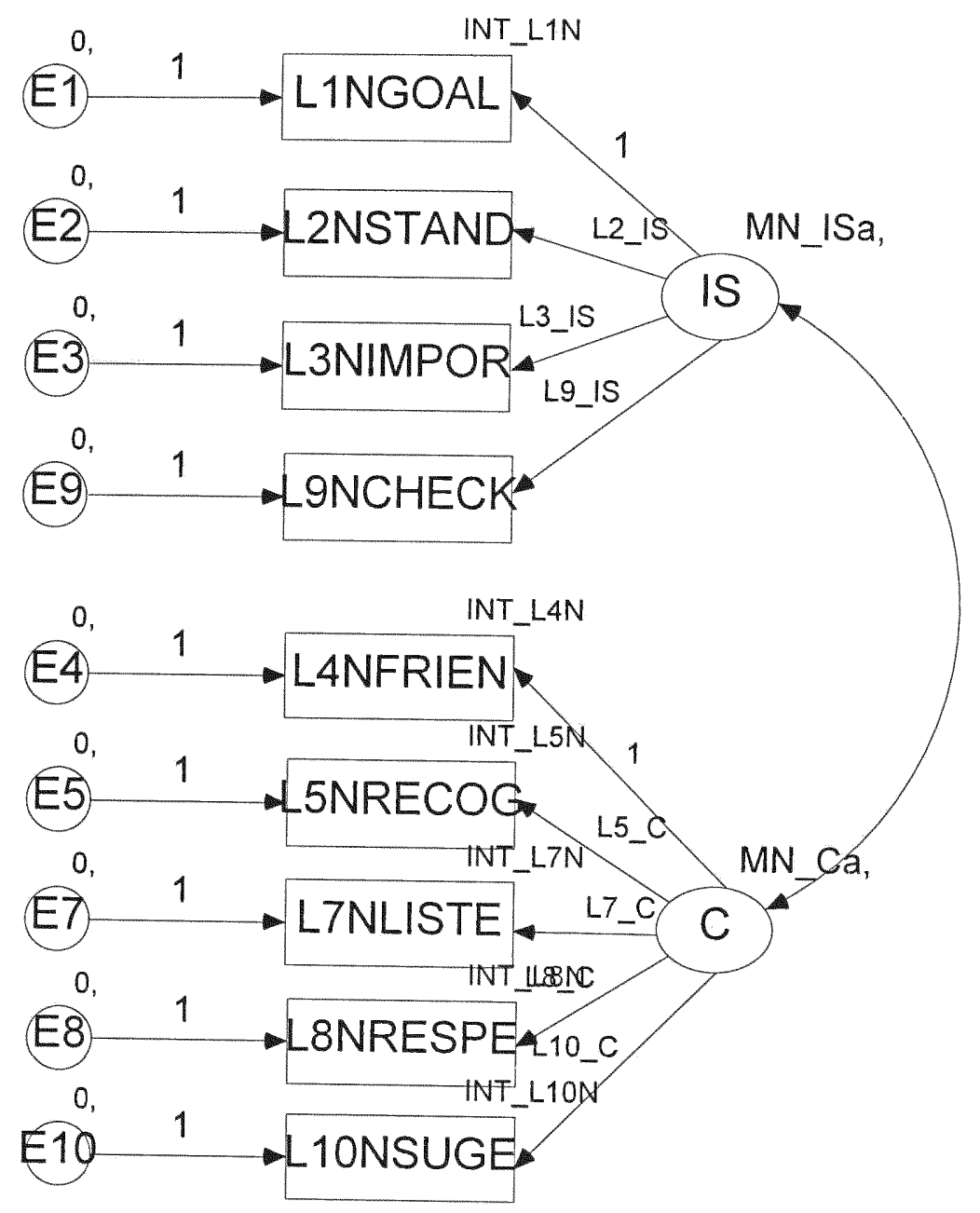

FIGURE 6: HYPOTHESIS 3 (C) EQUALITY OF INTERCEPTS CONSIDERATION MEASUREMENT MODEL 


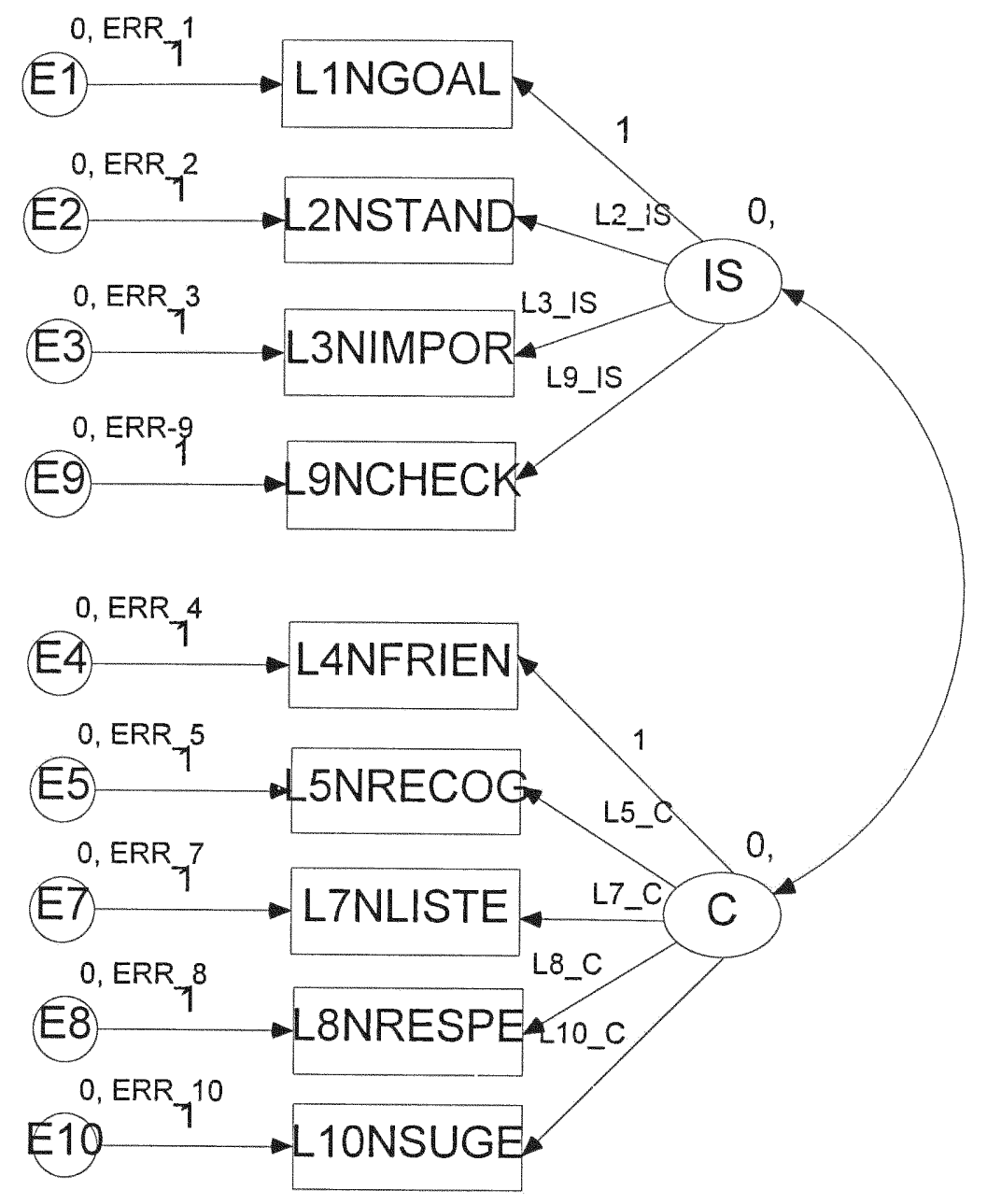

FIGURE 7: HYPOTHESIS 4

EQUALITY OF MEASUREMENT ERROR VARIANCES CONSIDERATION AND INITIATION OF STRUCTURE MEASUREMENT MODEL 


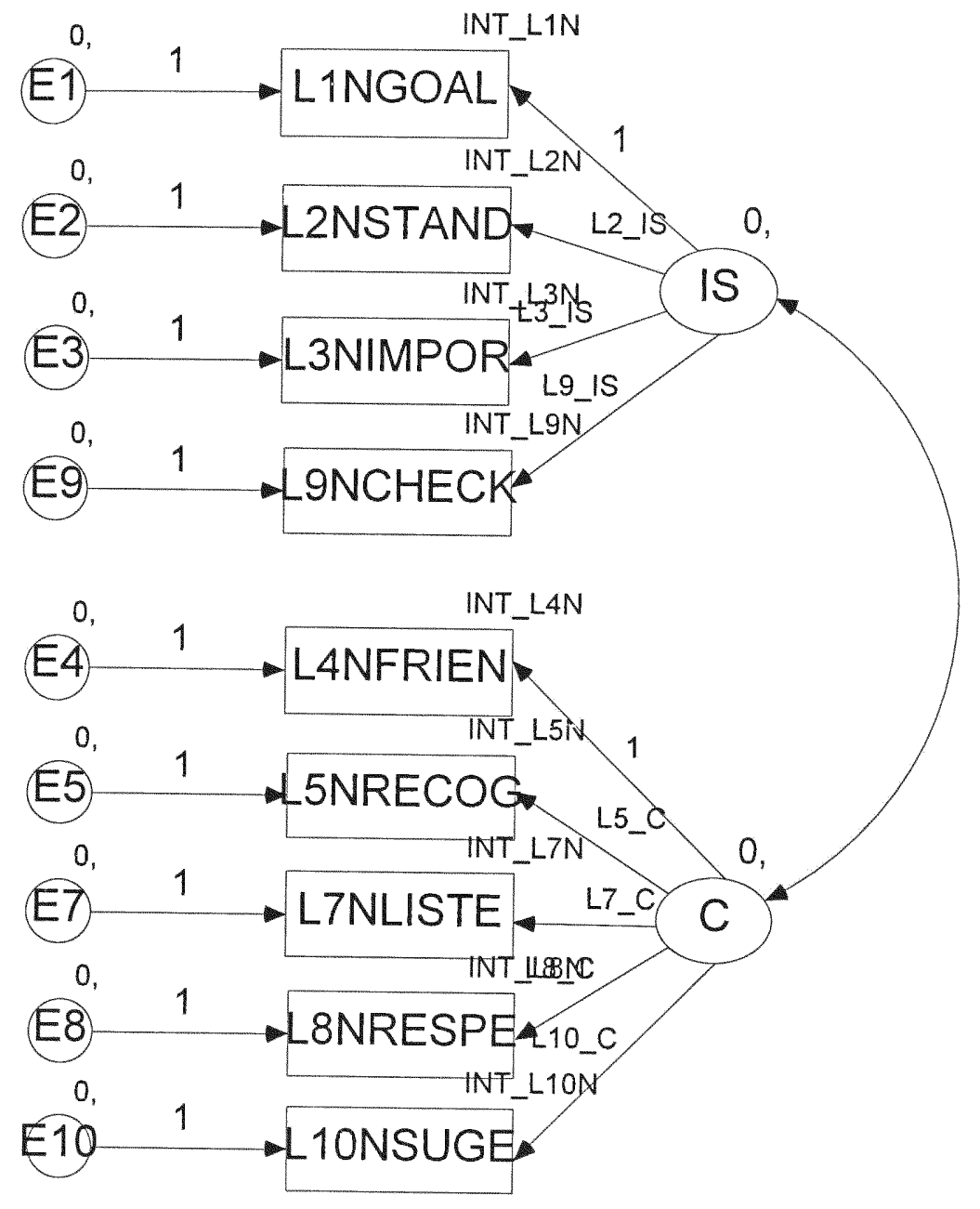

FIGURE 8: HYPOTHESIS 5

EQUALITY OF LATENT MEANS

MEASUREMENT MODEL

STEP 1 


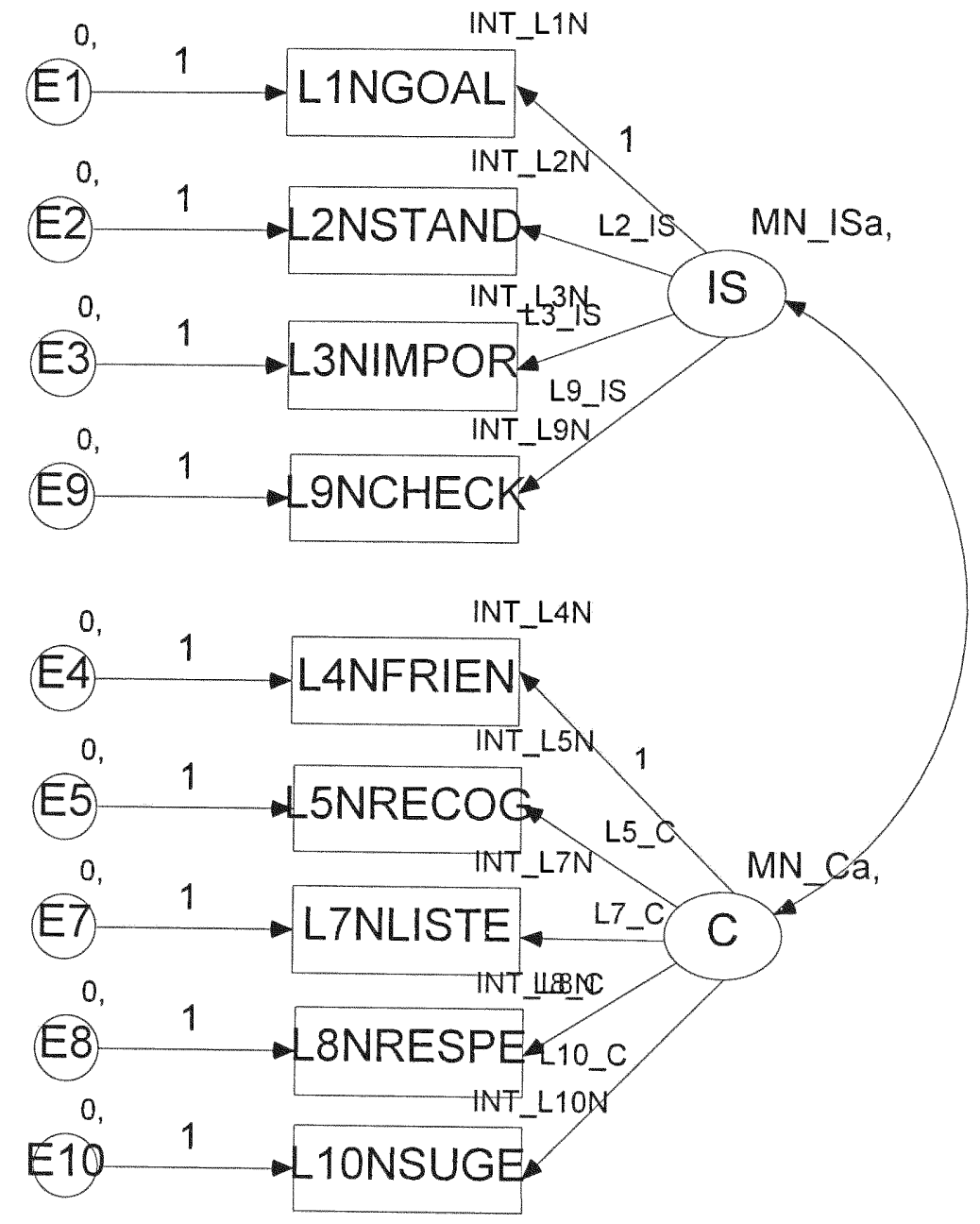

FIGURE 9: HYPOTHESIS 5

EQUALITY OF LATENT MEANS

MEASUREMENT MODEL

STEP 2 


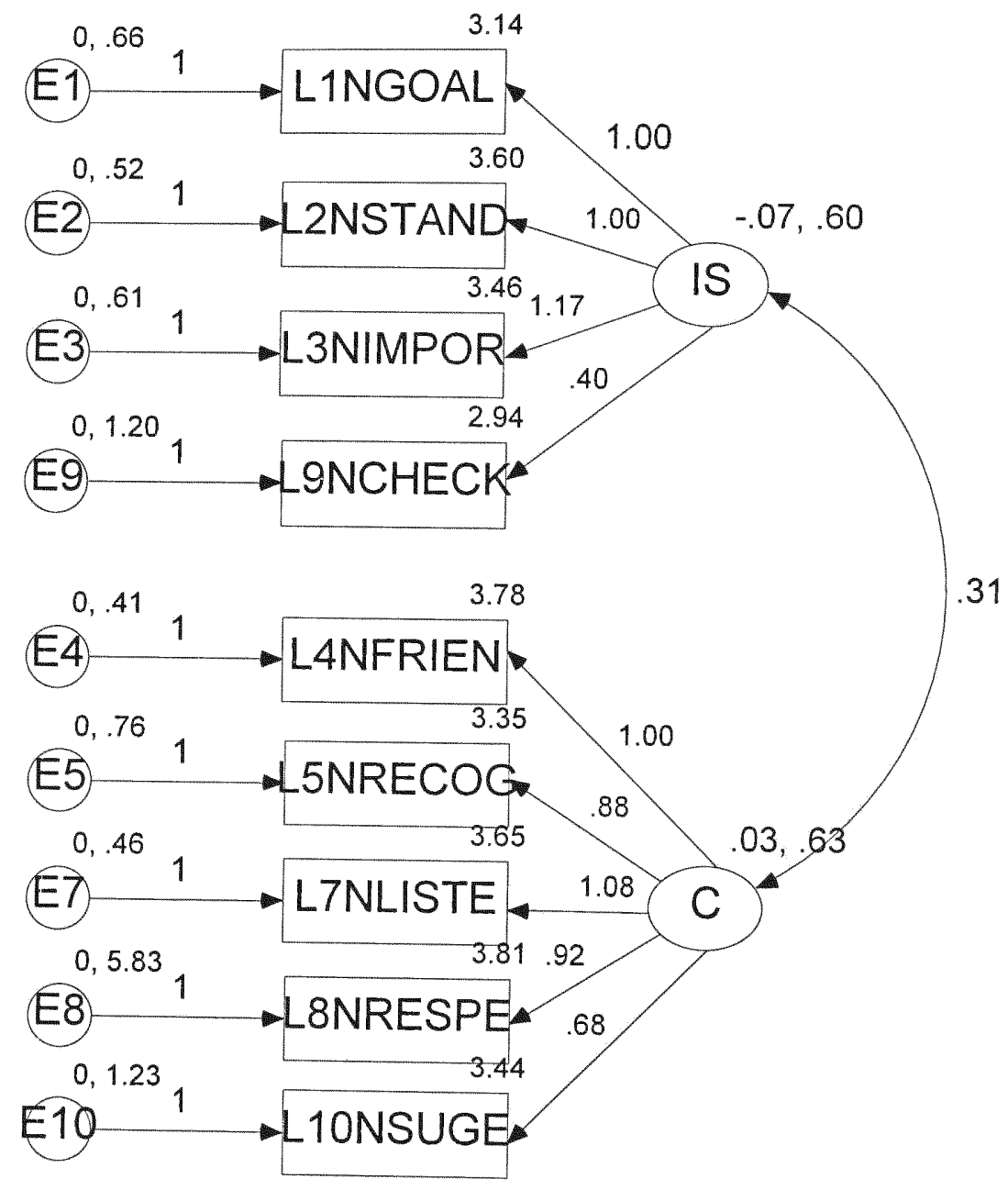

FIGURE 10: AUSTRALIA

EQUALTIY OF LATENT MEANS

STEP 1 


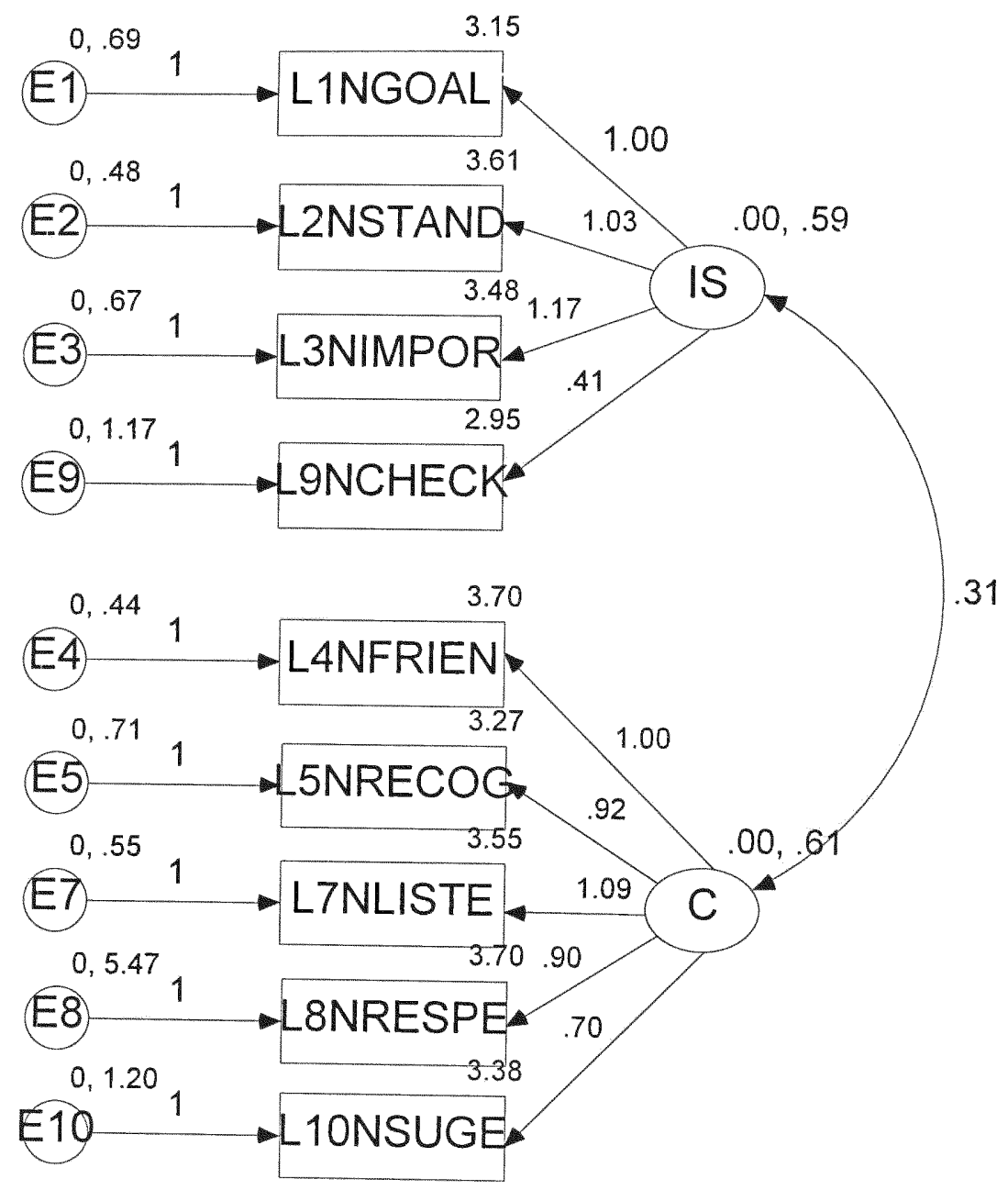

FIGURE 11: AUSTRALIA

EQUALITY OF LATENT MEANS

STEP 2 


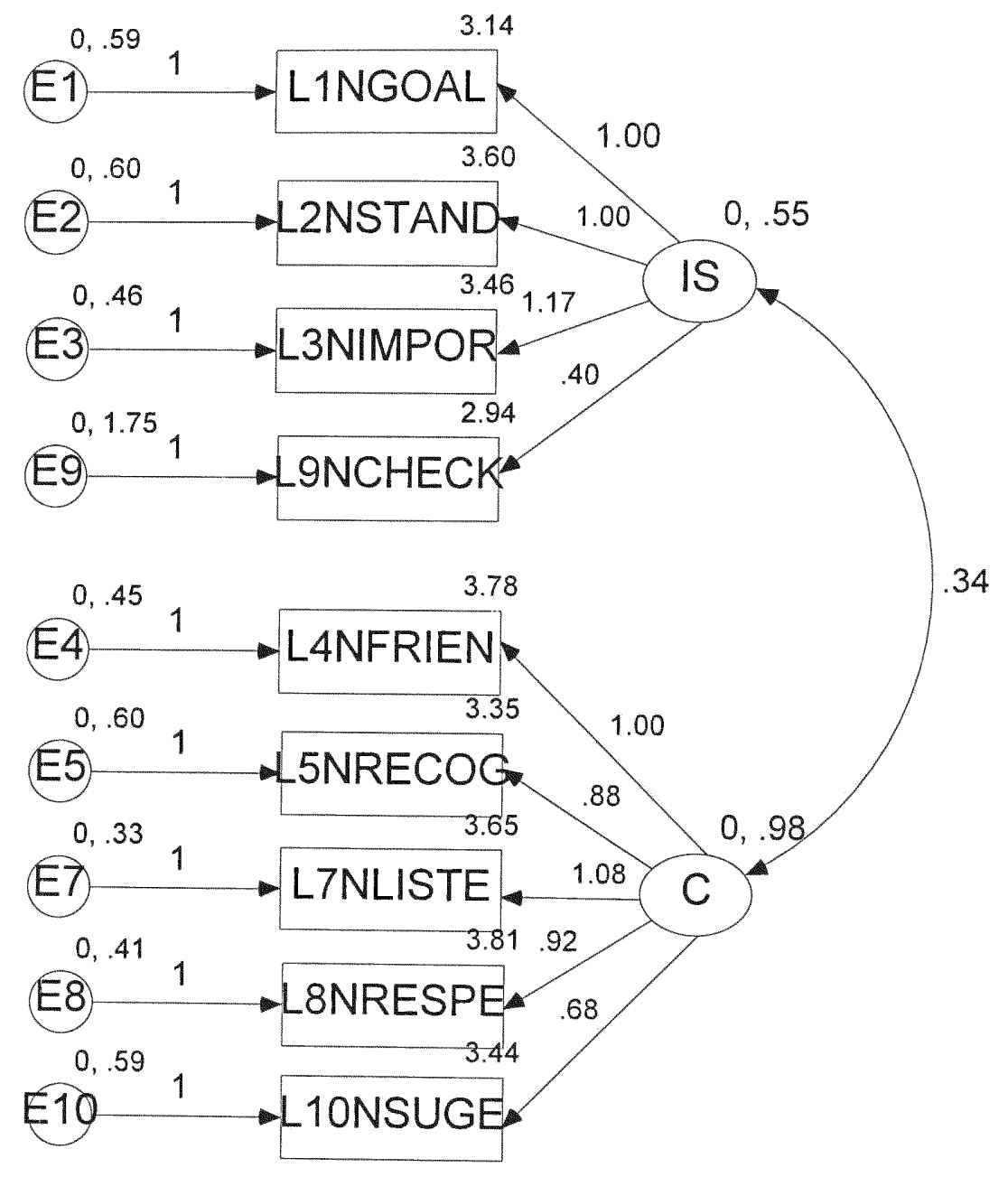

FIGURE 12: UNITED STATES

EQUALITY OF MEANS

STEP 1 


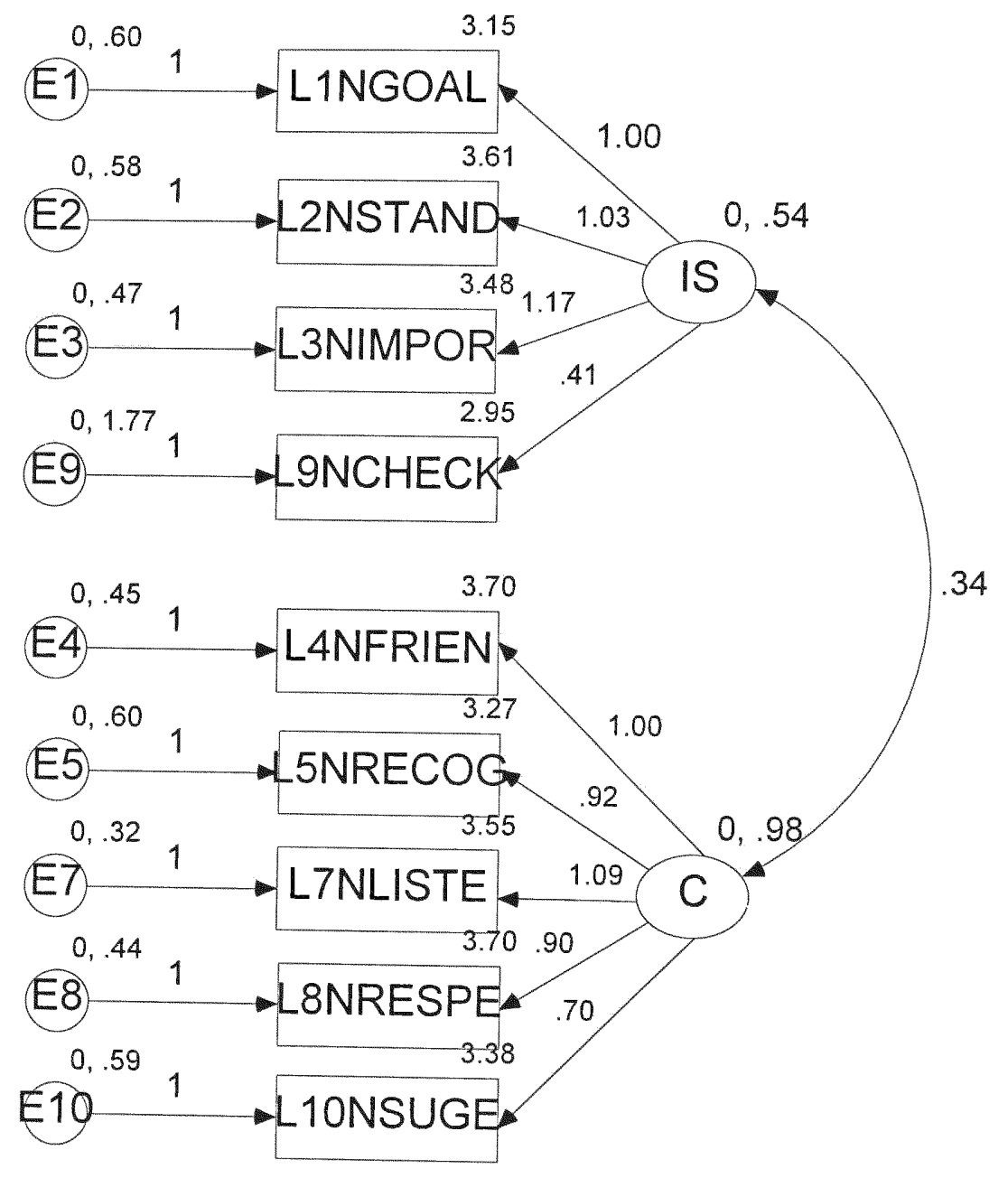

FIGURE 13: UNITED STATES

EQUALITY OF MEANS

STEP 2 


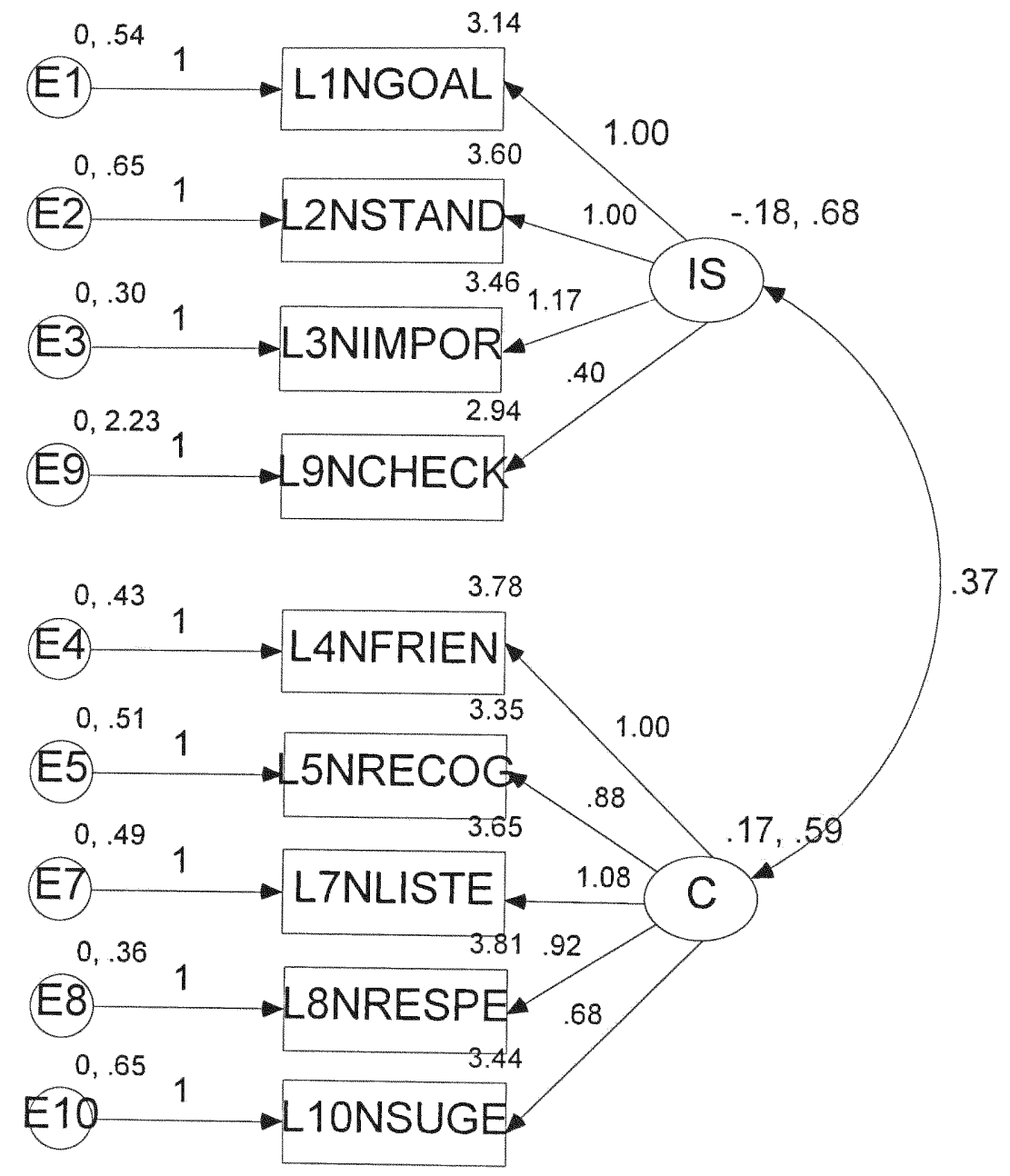

FIGURE 14: CANADA EQUALITY OF MEANS

STEP 1 


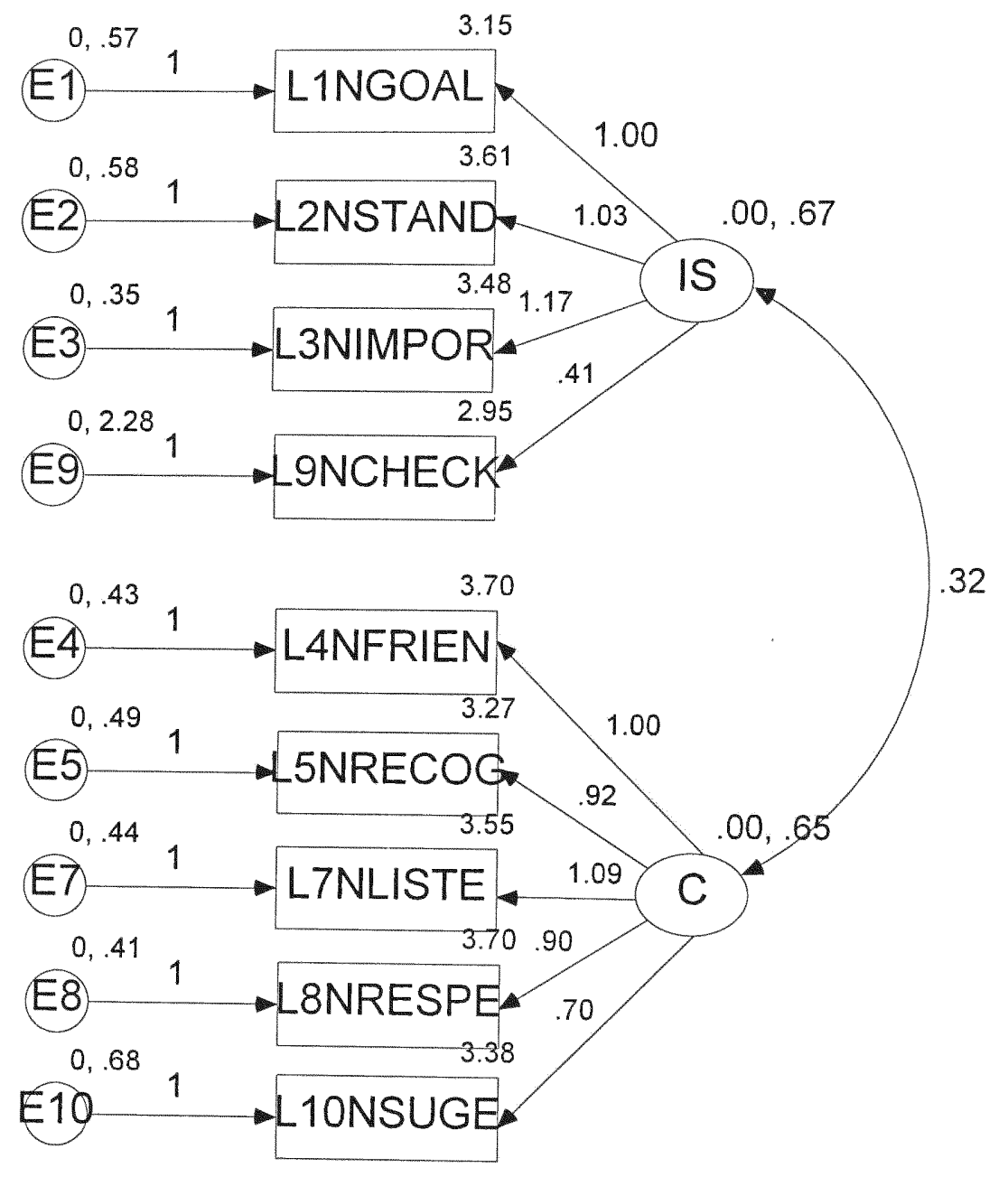

FIGURE 15: CANADA EQUALITY OF MEANS

STEP 2 


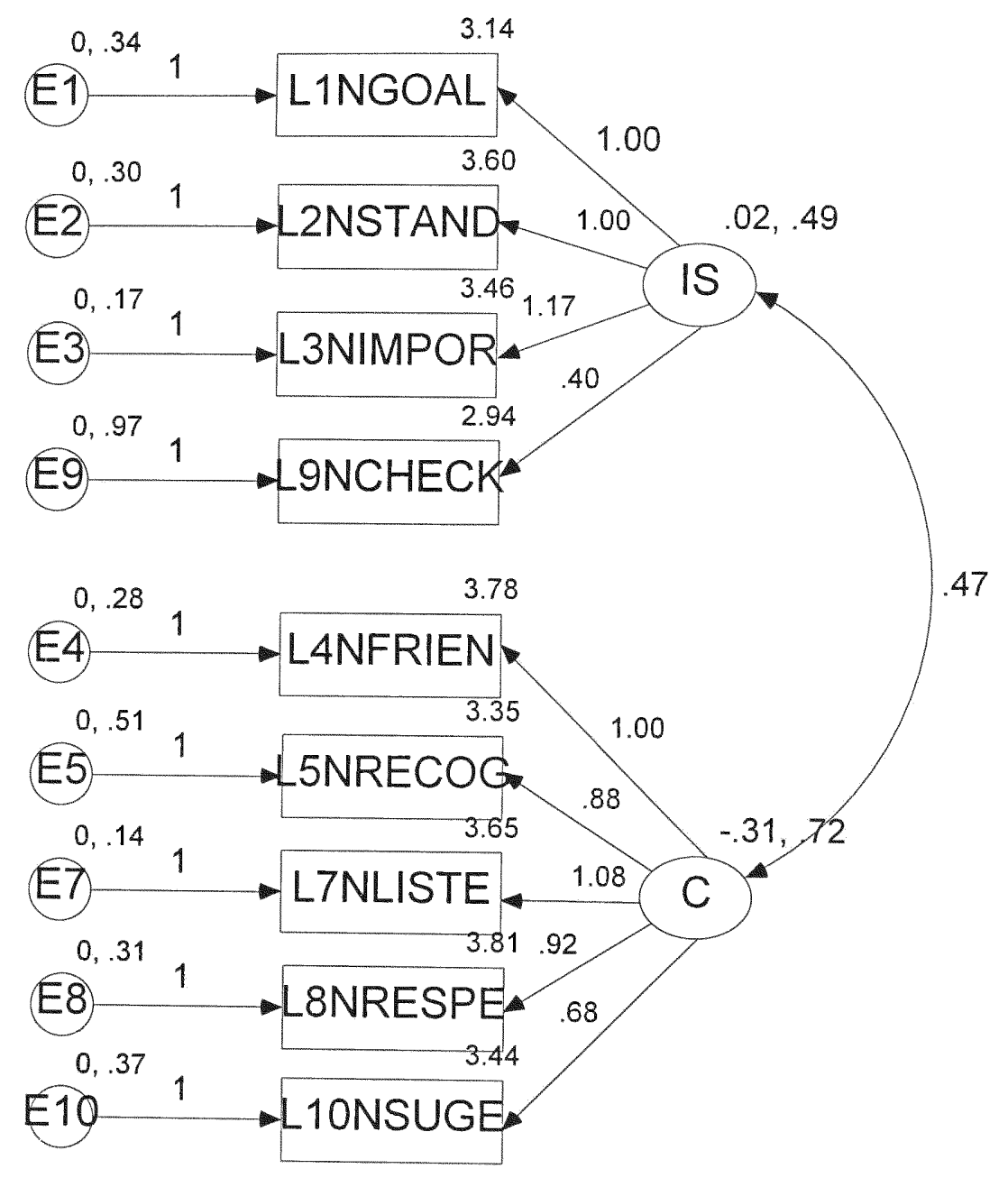

FIGURE 16: CHINA

EQUALITY OF MEANS

STEP 1 


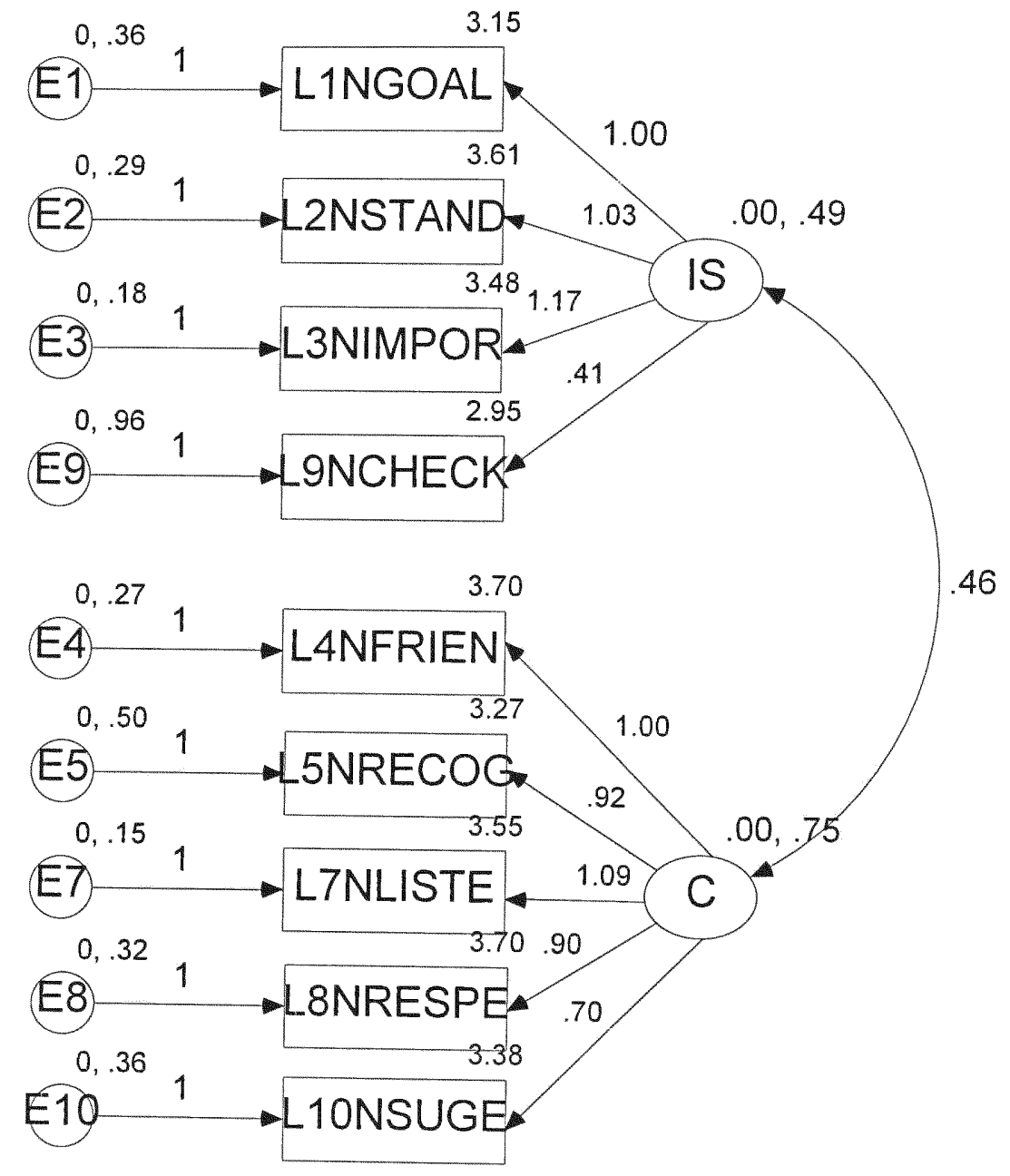

FIGURE 17: CHINA EQUALITY OF MEANS STEP 2 


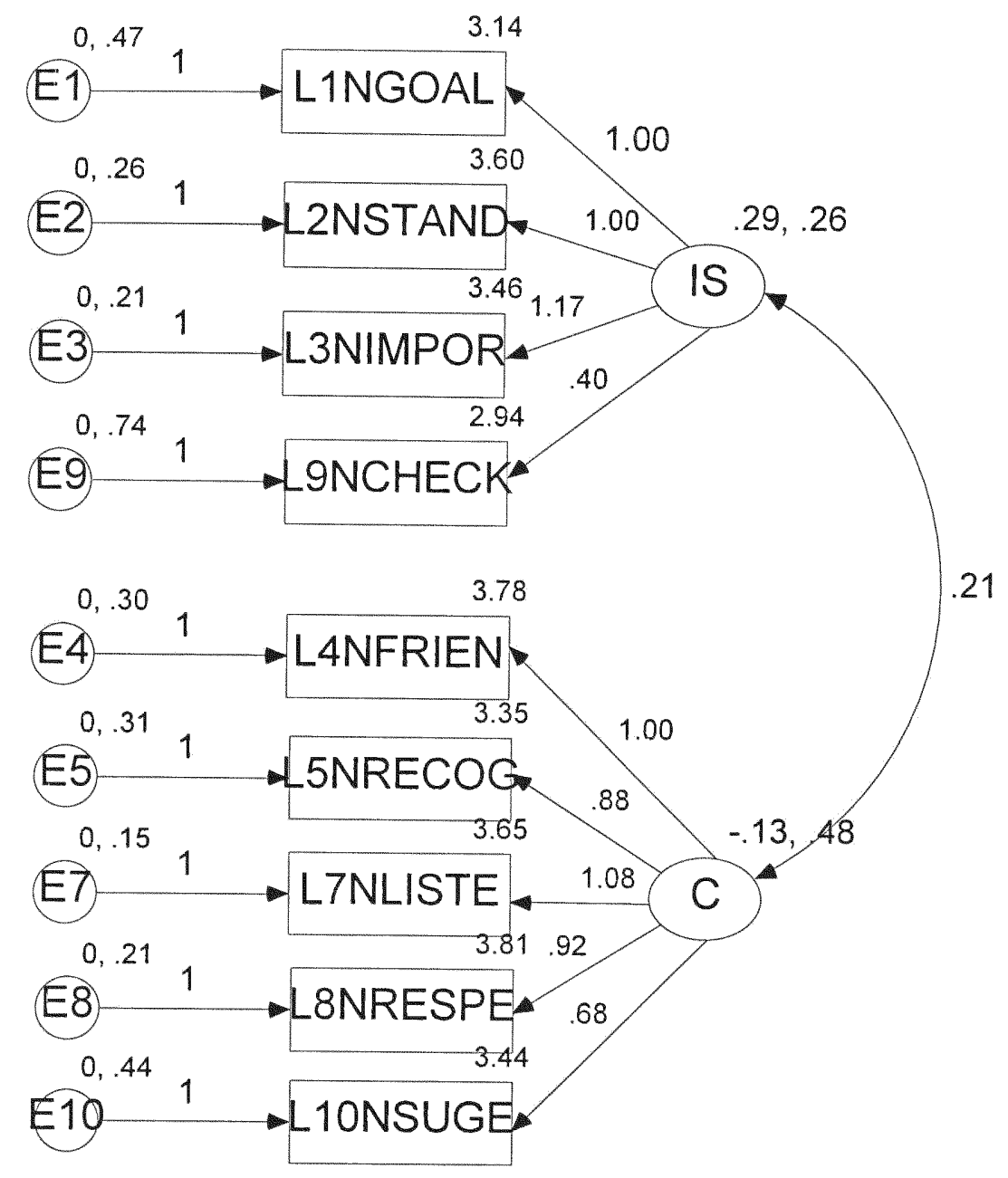

FIGURE 18: TAIWAN EQUALITY OF MEANS

STEP 1 


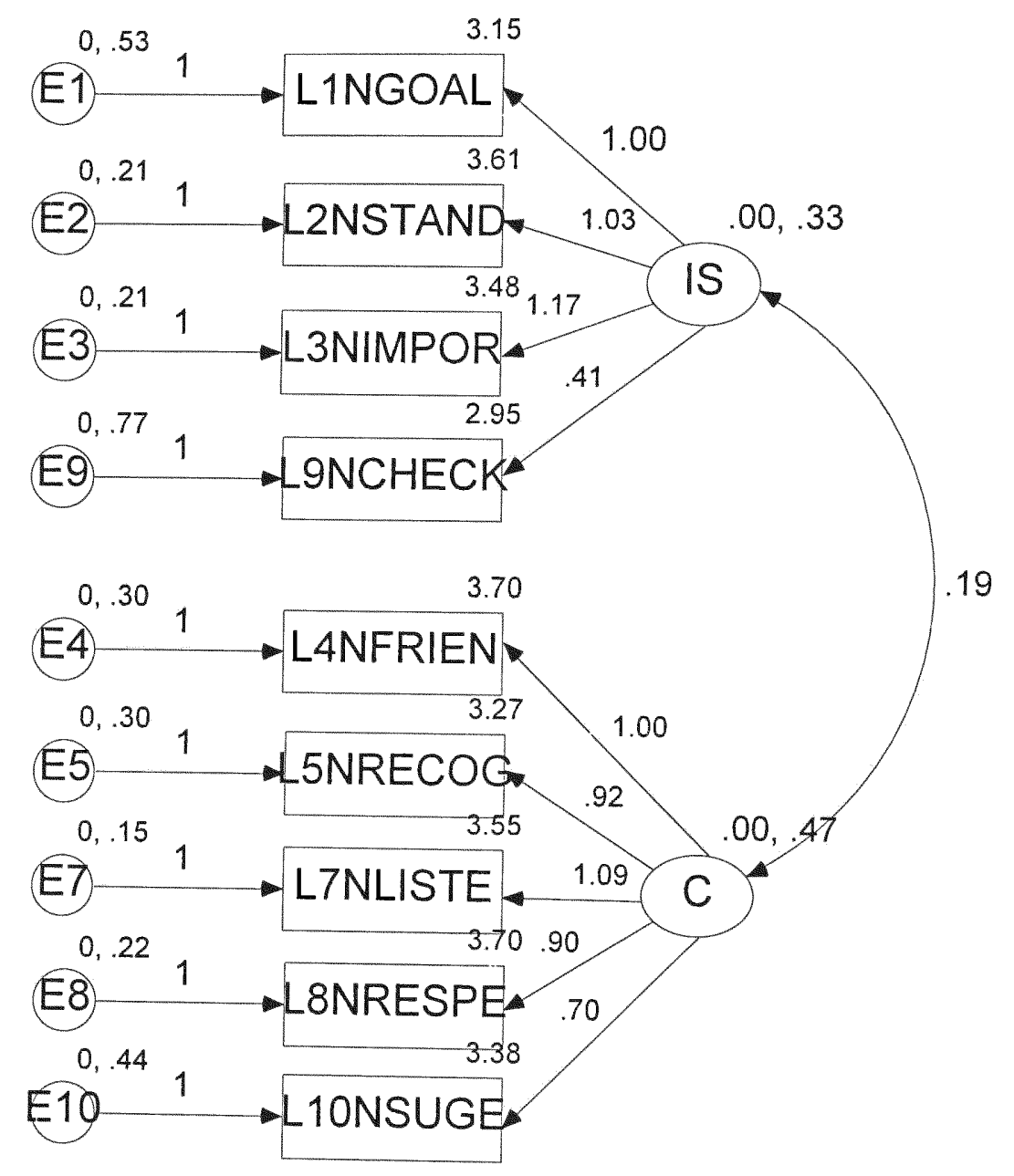

FIGURE 19: TAIWAN EQUALITY OF MEANS STEP 2 


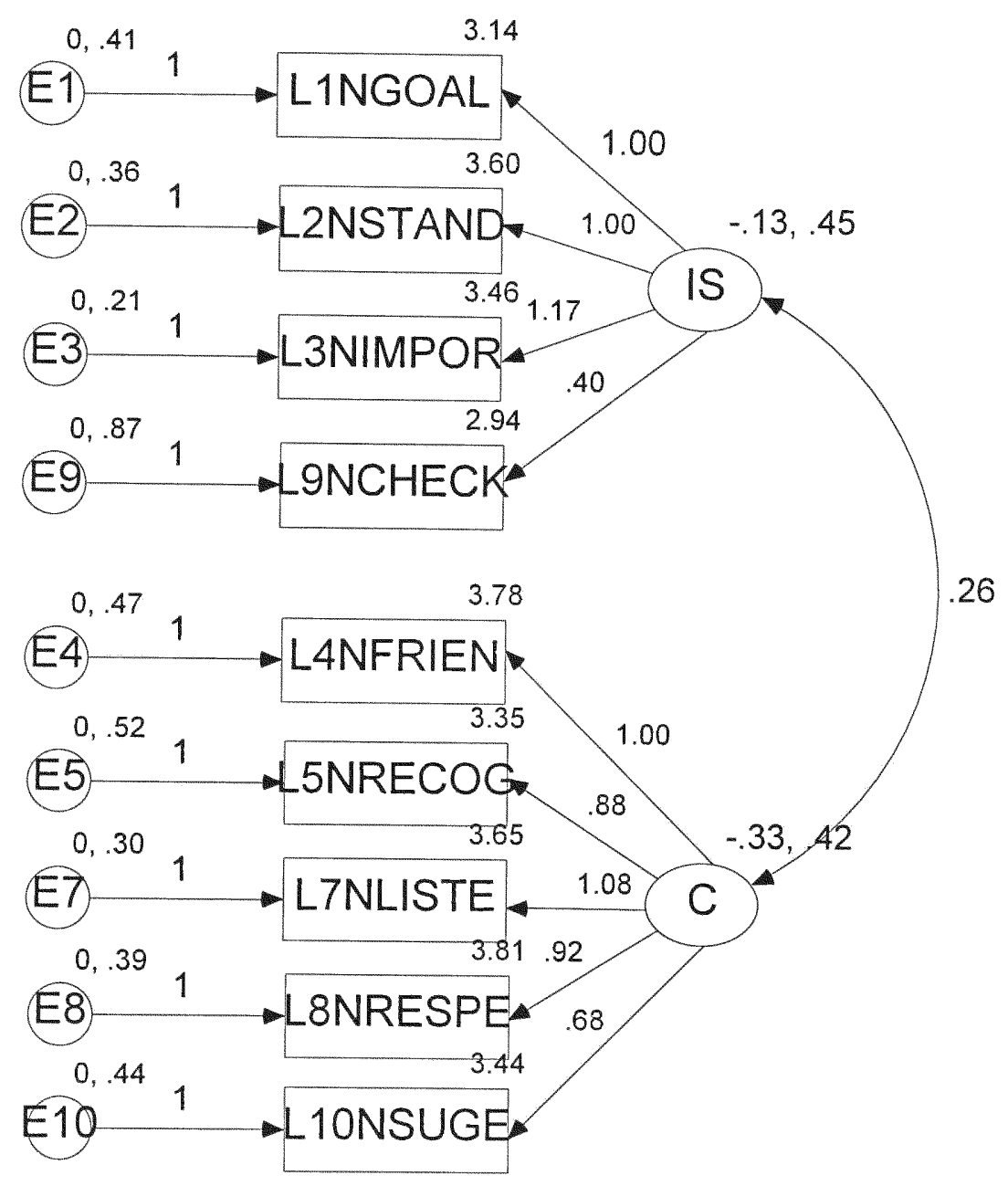

FIGURE 20: JAPAN

EQUALITY OF MEANS

STEP 1 


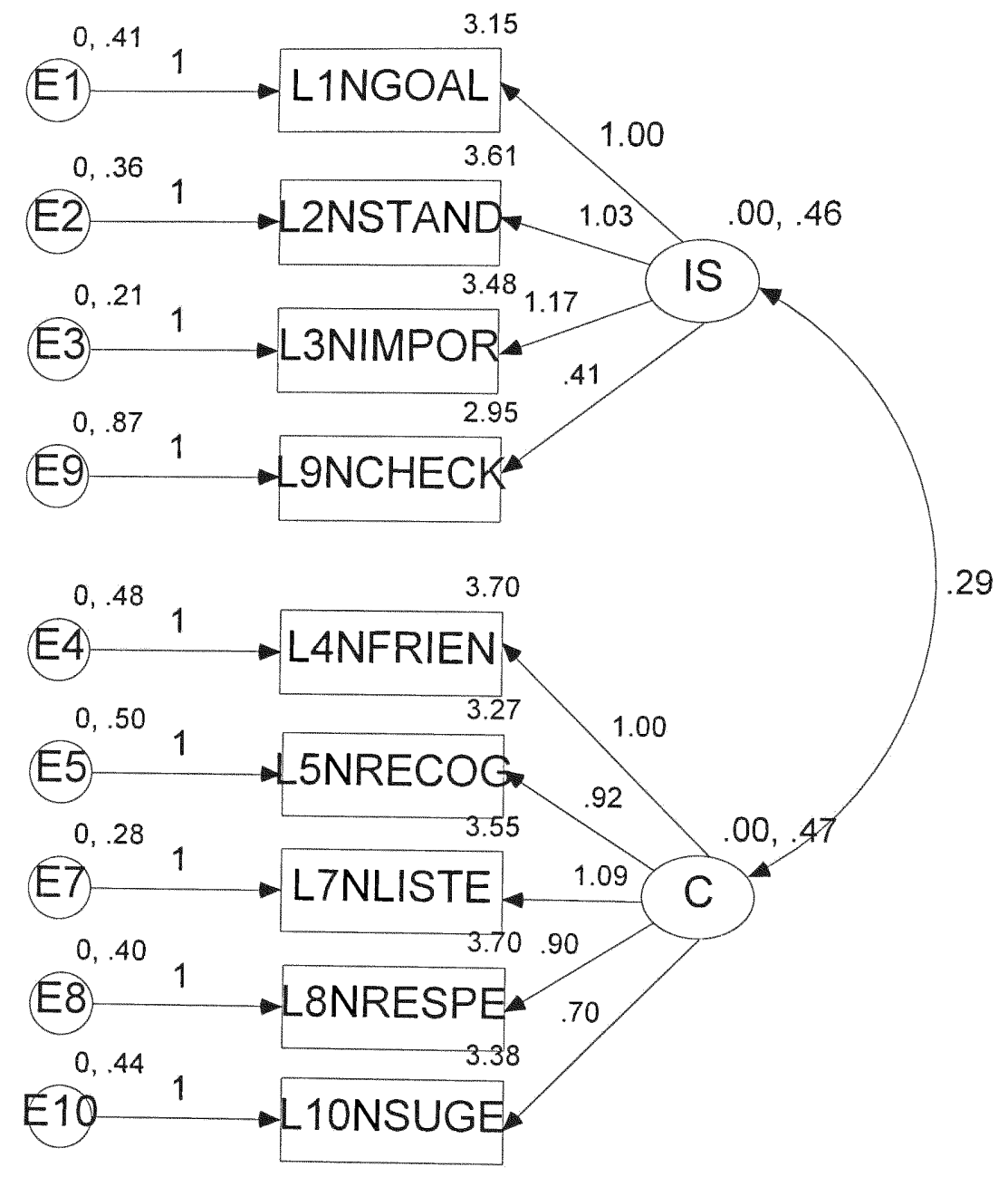

FIGURE 21: JAPAN EQUALITY OF MEANS

STEP 2 


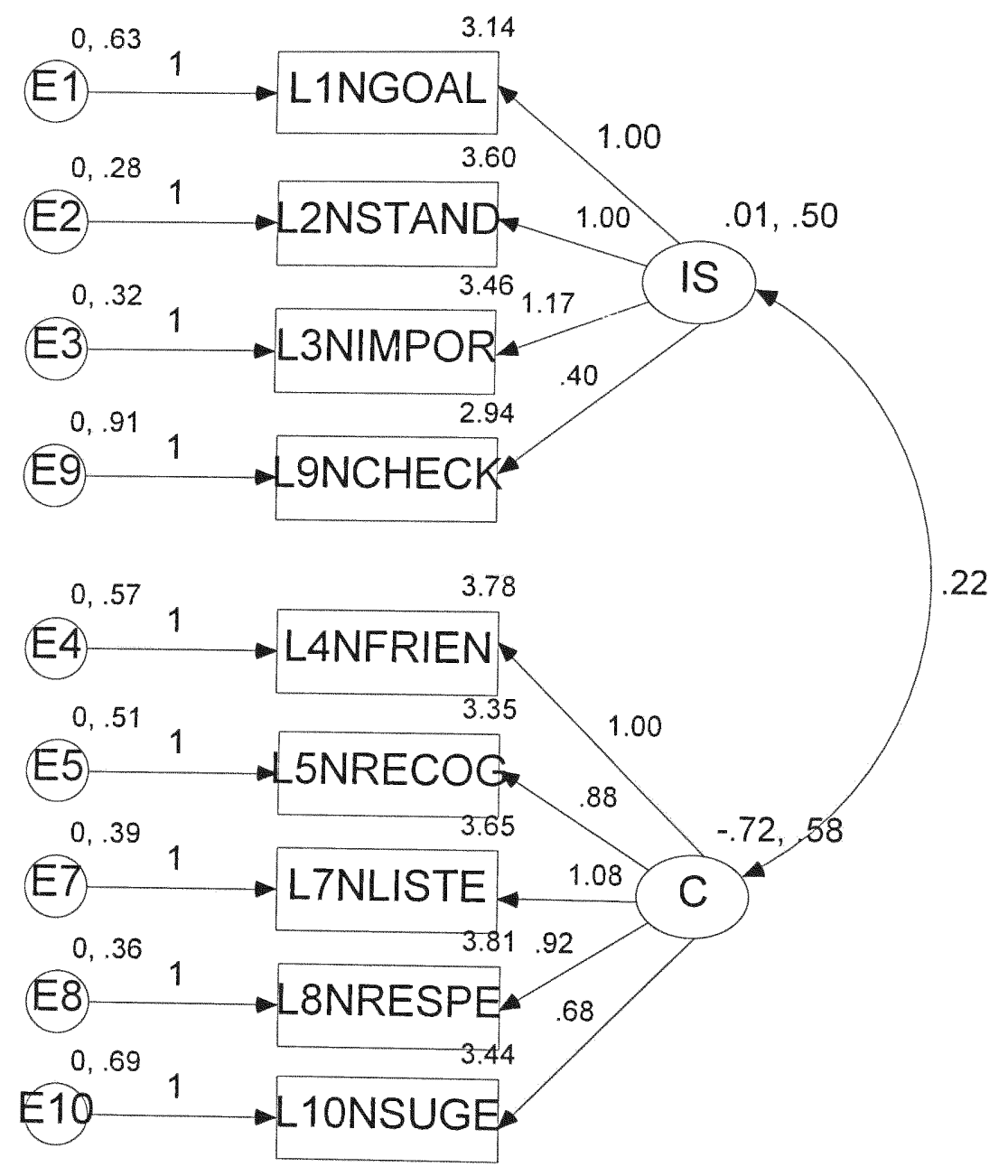

FIGURE 22: KOREA EQUALITY OF MEANS

STEP 1 


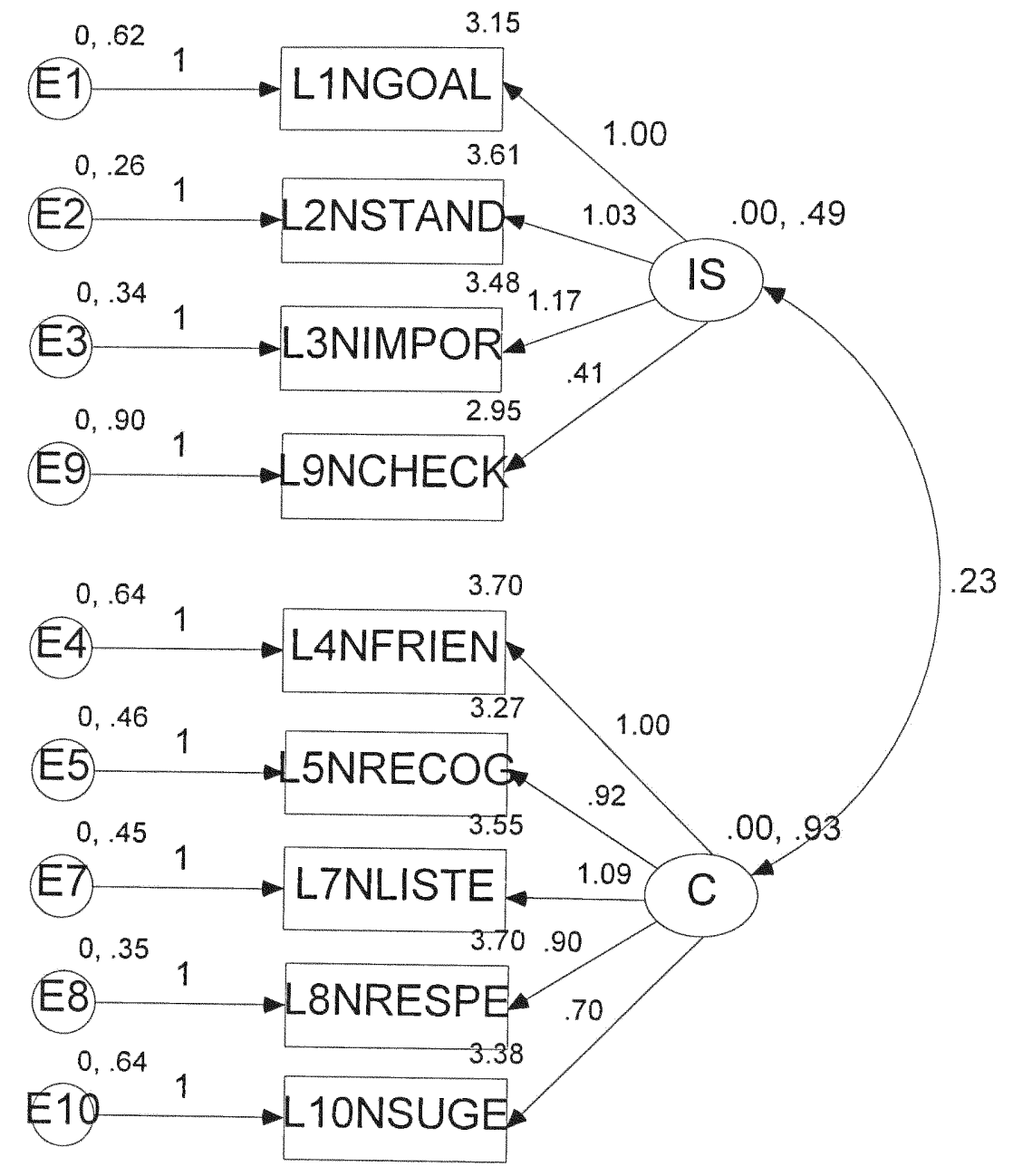

FIGURE 23: KOREA EQUALITY OF MEANS STEP 2 


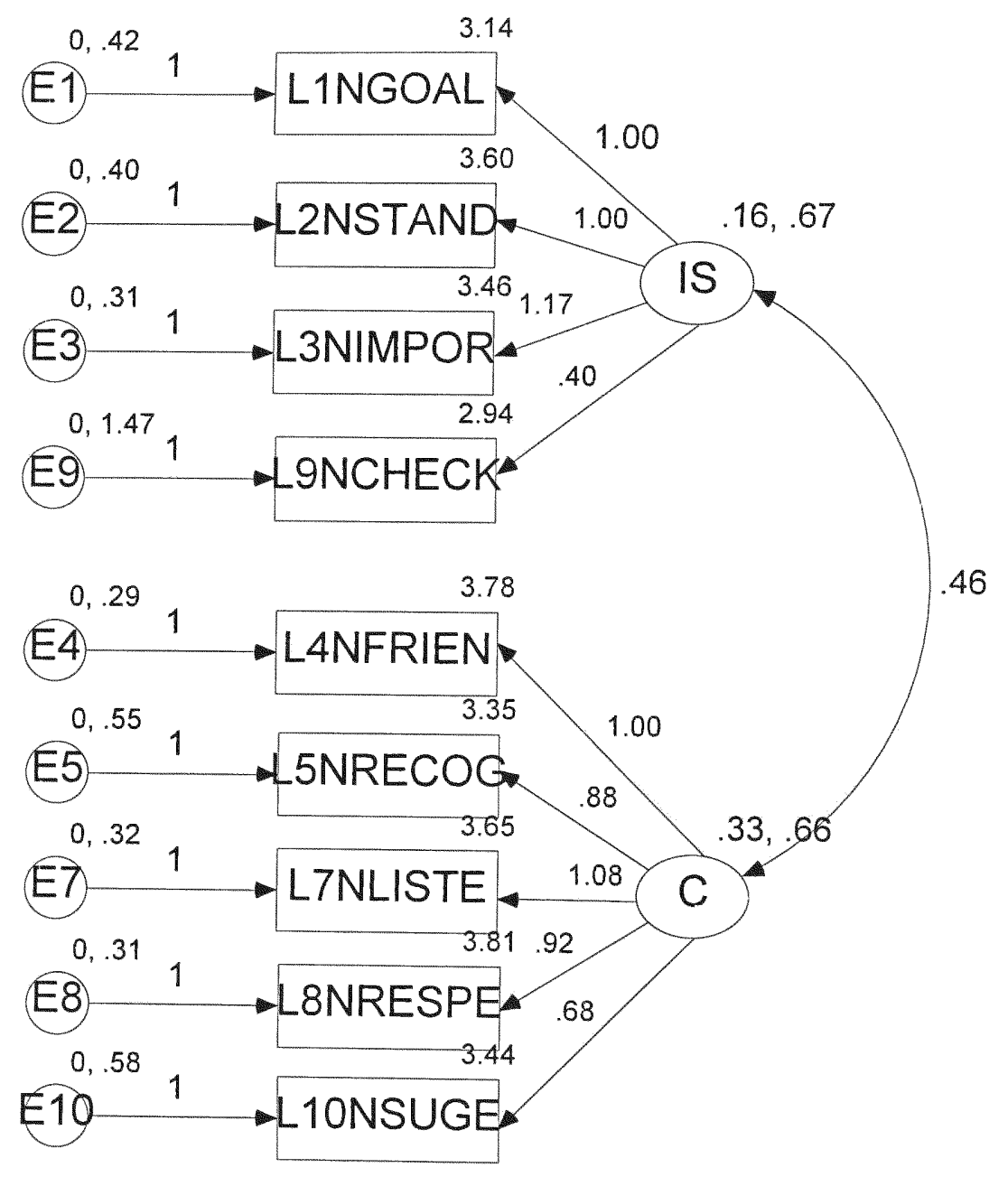

FIGURE 24: PHILIPPINES

EQUALITY OF MEANS

STEP 1 


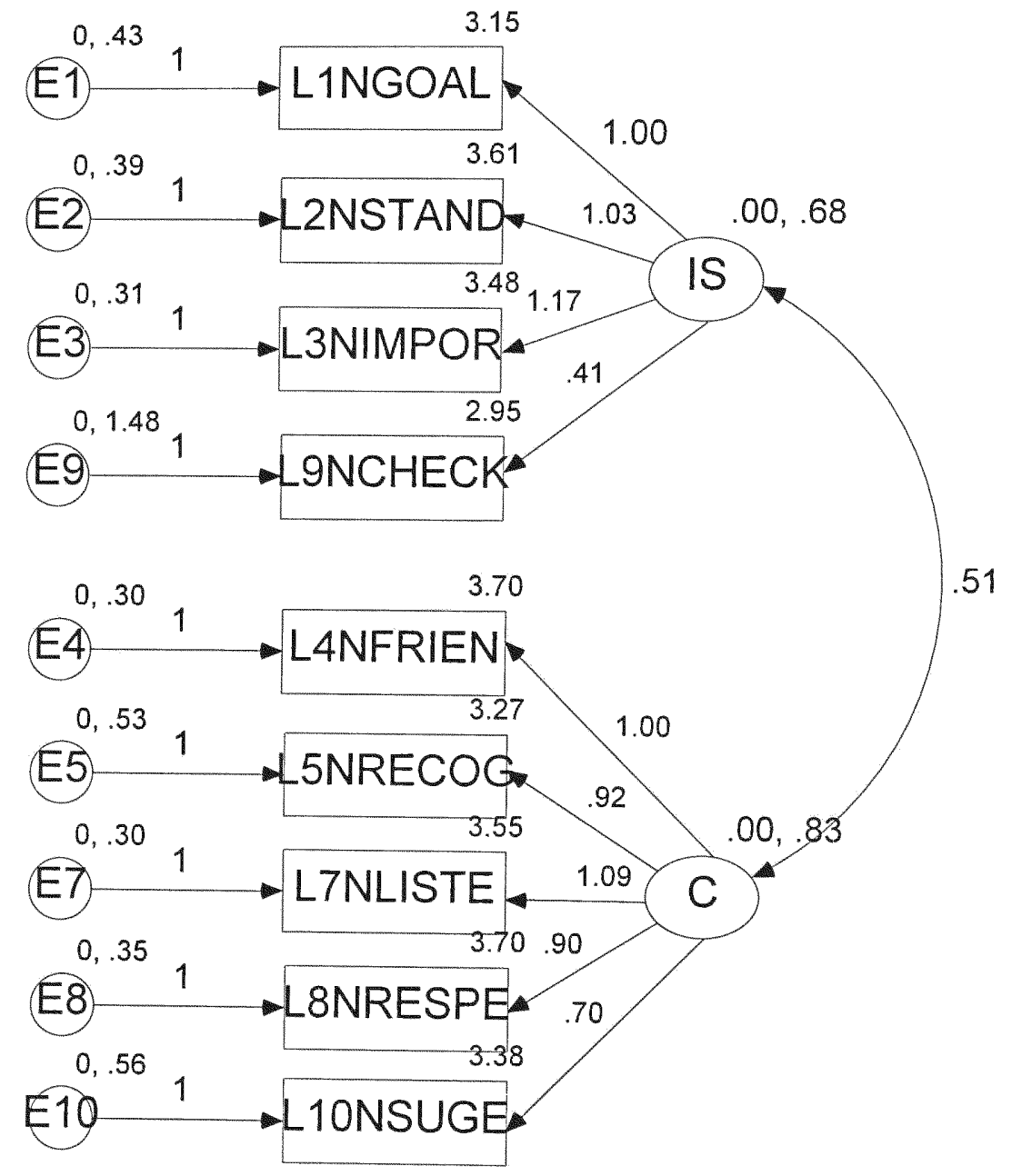

FIGURE 25: PHILIPPINES

EQUALITY OF MEANS

STEP 2 


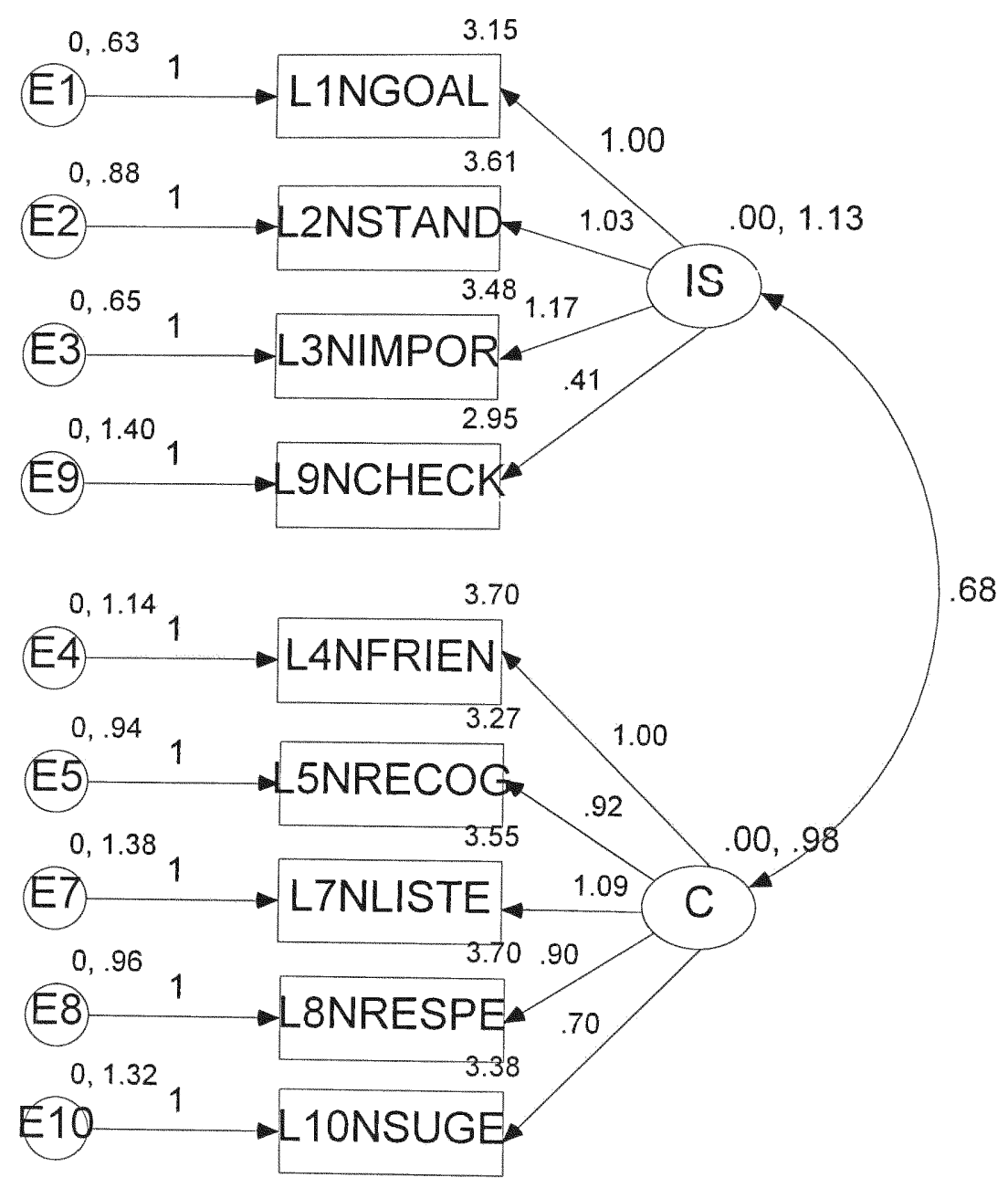

FIGURE 26: GULF REGION EQUALITY OF MEANS STEP 1 


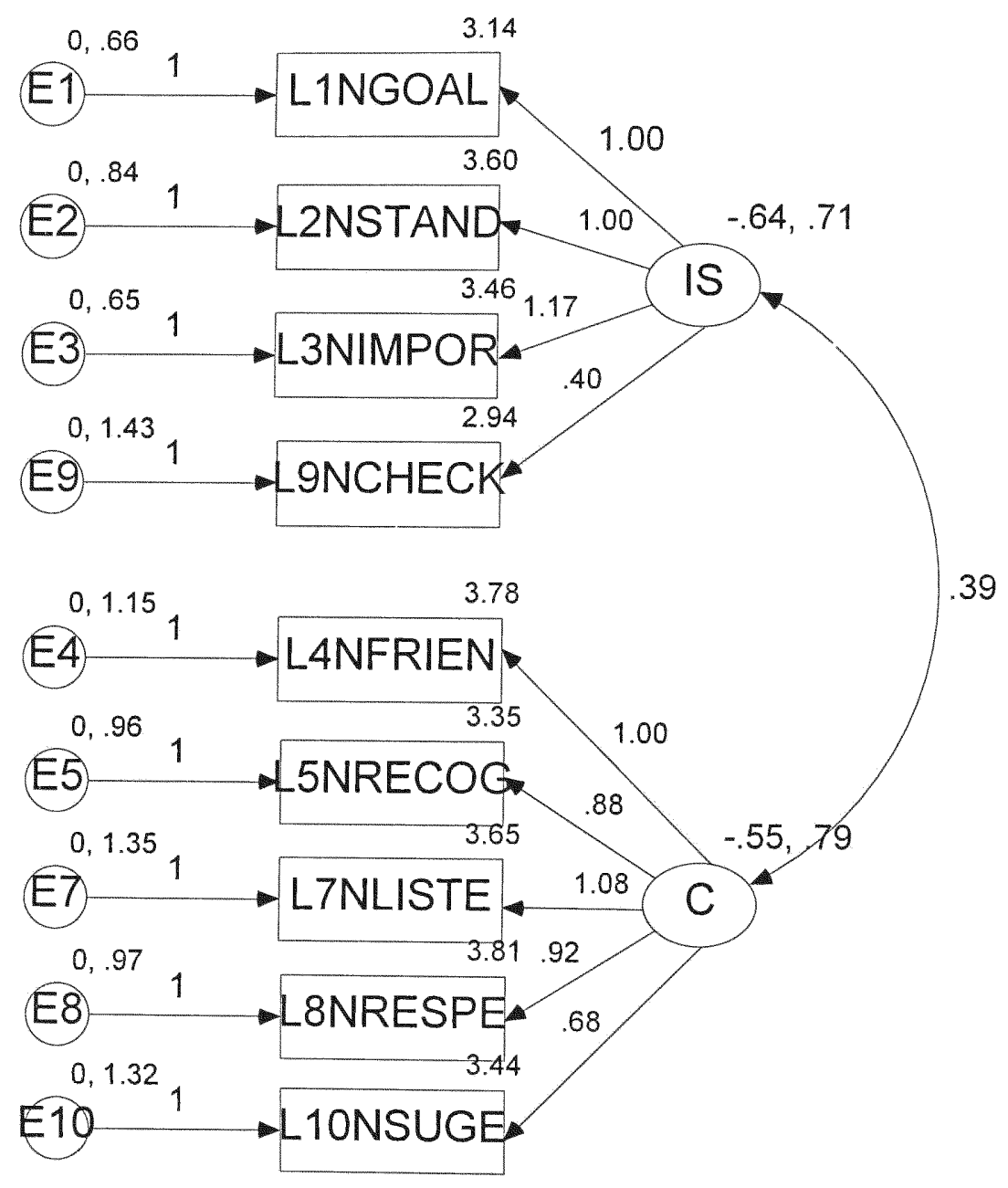

FIGURE 27: GULF REGION

EQUALITY OF MEANS

STEP 2 


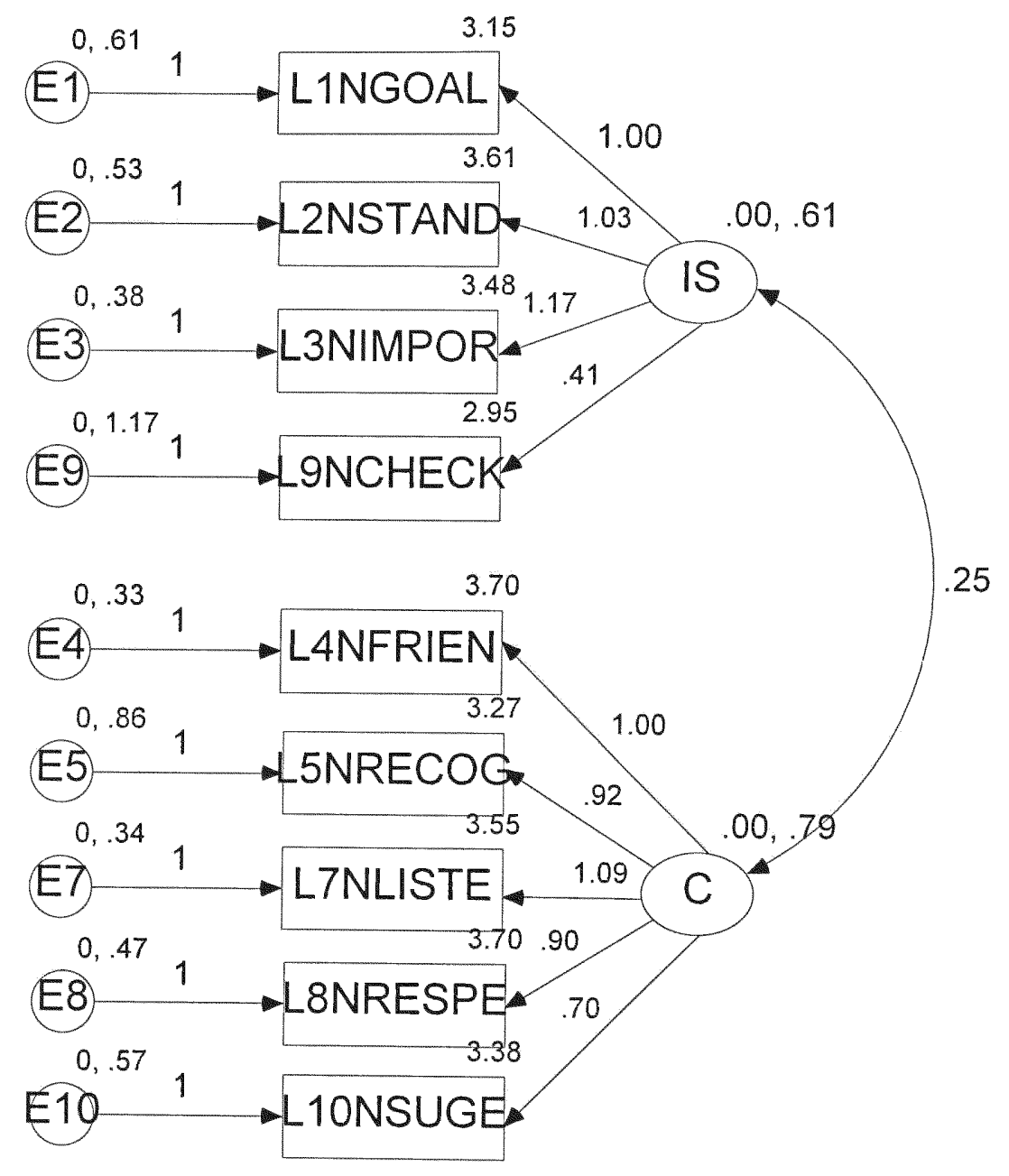

FIGURE 28: LATIN AMERICA EQUALITY OF MEANS

STEP 1 


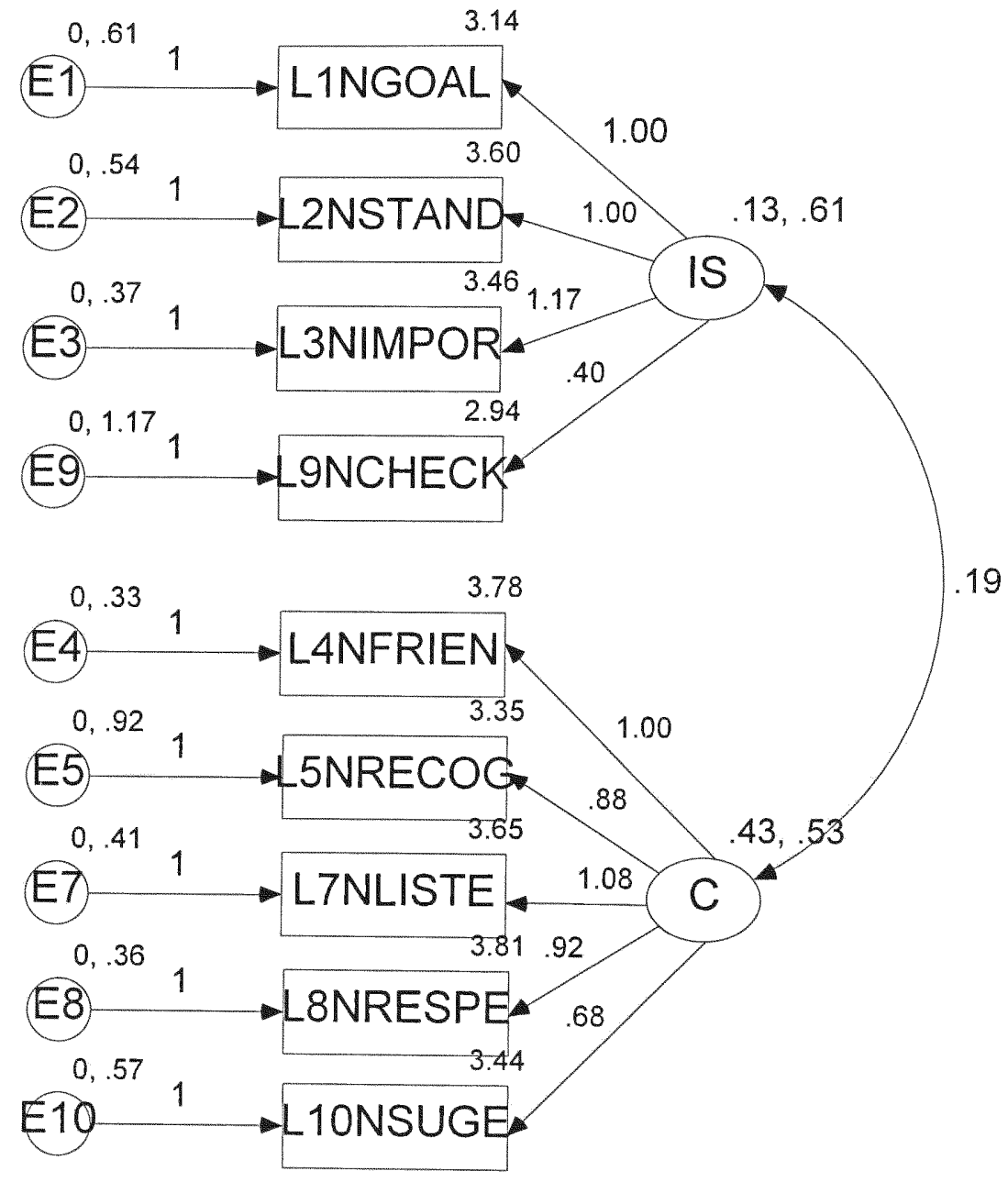

FIGURE 29: LATIIN AMERICA EQUALITY OF MEANS STEP 2 


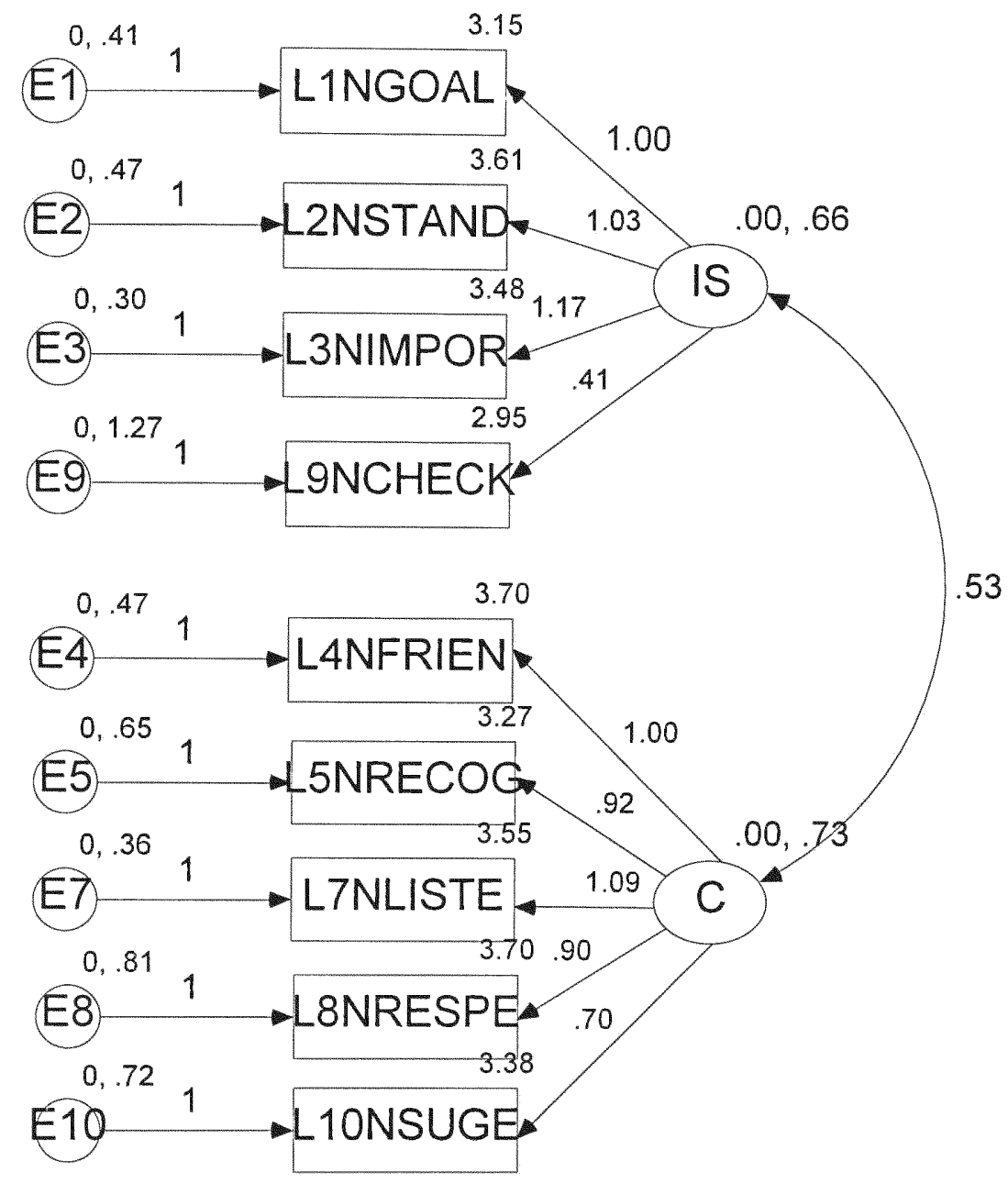

FIGURE 30: MEXICO

EQUALITY OF MEANS

STEP 1 


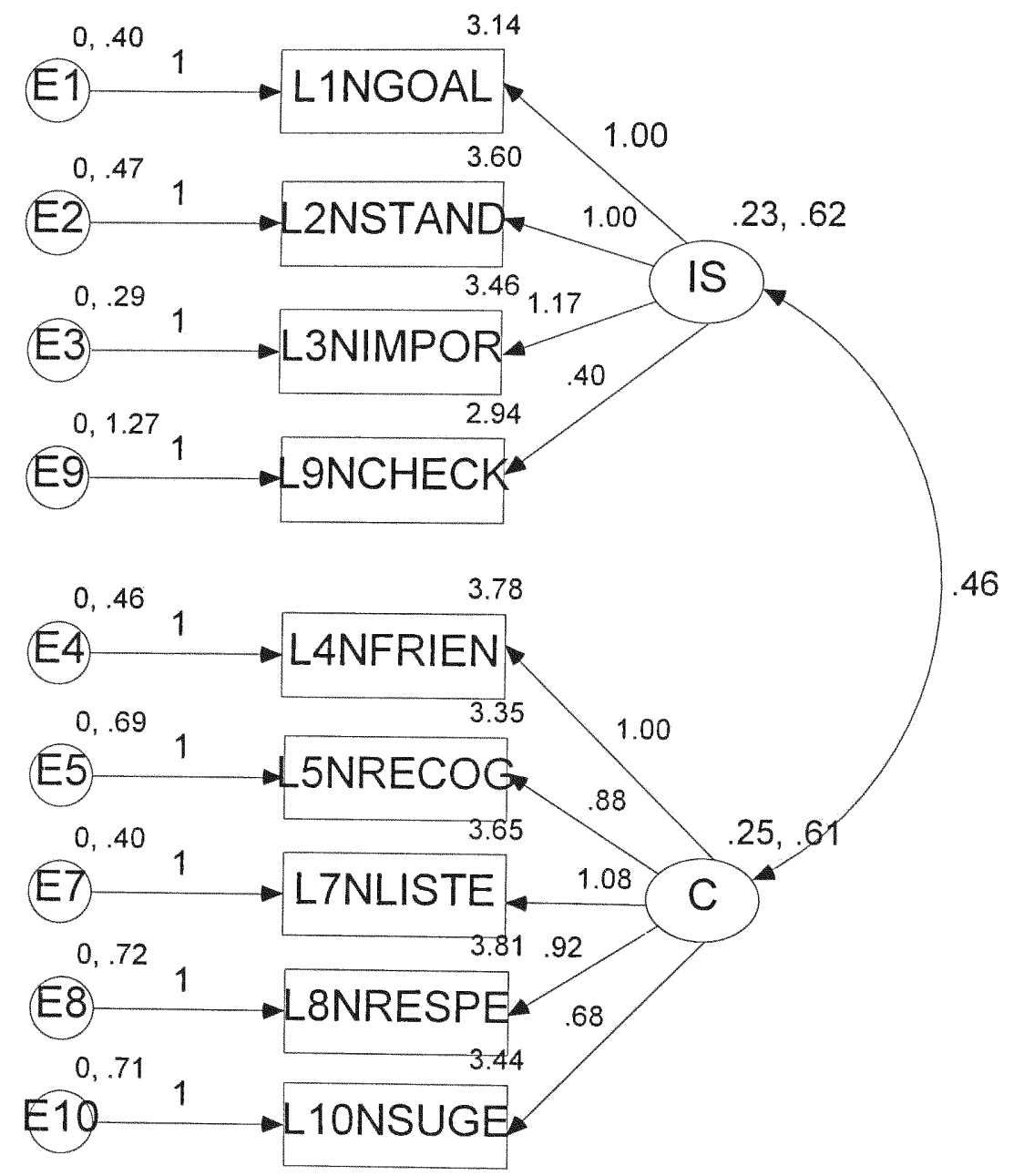

FIGURE 31: MEXICO

EQUALITY OF MEANS

STEP 2 


\section{REFERENCES}

Adler, N.J. (1984). Understanding the ways of understanding: Cross-cultural management methodology reviewed. Advances in International Comparative Management 1:31-67.

Al-Gattan A. (1985). Test of the Path-Goal Theory of leadership in the multinational domain. Group and organizational Studies 10(4): 429-445.

Alston, J.P. (1989). Wa, guanxi and Inhwa: Managerial principles in Japan, China, and Korea. Business Horizons, March-April, 26-31.

Anderson, L.R. (1983). Management of the mixed-cultural work group. Organizational Behavior and Human Performance, 31, 303-330.

Arbucke, J.L. and Wothke, W. (1999). AMOS 4.0 User's Guide. SmallWaters Corporation.

Ashour, A.S. (1973). Further discussion of Fiedler's contingency model of leadership effectiveness: an evaluation. Organizational Behavior and Human Performance, 9: 339355.

Ayman, R., Chemers, M.M. and Fiedler, F. E. (1998). The contingency model of leadership effectiveness: Its level of analysis. In Dansereau, F. \& Yammarino, F. J. (Eds.), Leadership: The Multiple-Level Approaches, Classical and New Wave, 24, Part A, 73-96. Stamford, CT: JAI Press Inc.

Ayman, R. and Chemers, M.M. (1982). The relationship of managerial behavior to effectiveness and satisfaction in Mexico. Paper, International Congress of Applied Psychology. Edinburgh.

Ayman, R. and Chemers, M.M. (1983). Relationship of supervisory behavior ratings to work group effectiveness and subordinate satisfaction among Iranian managers. Journal of Applied Psychology, 68 (2), 338-341.

Bagozzi, R.P., and Yi, Y. (1989). On the use of structural equation models in experimental designs. Journal of marketing Research, 26, 271-284.

Bales, R. F. (1950). Interaction process analysis. Reading, MA: Addison-Wesley.

Bales, R.F. and Slater, P.E. (1955). Role differentiation is small decision-making groups. In T. Parsons \& R.F. Bales (Eds.), Family, socialization, and interaction processes. New York, Free Press. 
Bass, B.M. (1962). Orientation inventory. Palo Alto, CA: Consulting Psychologists Press.

Bass, B.M. (1985). Leadership and performance beyond expectation. New York: Free Press.

Bass, B.M. (1990). Bass and Stogdill's Handbook of Leadership. 3d ed. New York: Free Press.

Bass, B.M. (1991). Is there universality in the full range model of leadership? Paper presented at the National Academy of Management Annual Meeting, Miami.

Bass, B.M. (1999). On the taming of charisma: a reply to Janice Beyer. Leadership Quarterly, 10(2), 541-553.

Bass, B.M. and Valenzi, E.R. (1974). Contingency aspects of effective management styles. In J.G. Hunt and L.L. Larsen (Eds.), Contingency approaches to leadership. Carbondale, IL: Southern Illinois University Press.

Bass, B.M., Valenzi, E.R., Farrow, D.L. and Solomon, R.J. (1975). Management style associated with organizational, task, personal, and interpersonal contingencies. Journal of Applied Psychology, 60, 720-729.

Bass, B.M., P.C. Burger, R. Doktor, and Barrett, G.V. (1979). Assessment of Managers: An International comparison. New York: Free Press.

Bass, B.M., D.A. Waldman, B.J. Avolio, and Bebb, M. (1979). Assessment of managers: An international comparison. New York: Free Press.

Bass, B.M. and Avolio, B.J. (1989). The mulifactor leadership questionnaire. Palo Alto, CA: Consulting Psychologiests Press.

Bass, B.M. and Avolio, B.J. (1993). Transformational leadership: A response to critiques. In M.M. Chemers and R. Ayman (eds.), Leadership theory and research: Perspectives and Directions, 49-80, San Diego, Academic Press.

Bennis, W. and Nanus, B. (1985). Leaders: The strategies for taking charge. New York: Harper \& Row.

Bensaou, M., Coyne, M. and Venkatraman, N. (1999). Testing metric equivalence in cross-national strategy research: An empirical test across the United States and Japan. Strategic Management Journal, 20, 671-689.

Bennett, M. (1977). Testing management theories cross-culturally. Journal of Applied Psychology, 62(5), 578-581. 
Bentler, P.M. (1980). Multivariate analysis with latent variables: Causal modeling. Annual Review of Psychology, 31, 419-456.

Berry, J.W. (1990). Imposed etics, emics and derived emics: Their conceptual and operational status in cross-cultural psychology. In T.N. Headland, K.L. Pike and M. Harris (Eds.), Emics and etics: Insider/outsider debate. Newbury Park, California: Sage Publications.

Berry, J.W., Poortinga, Y.H., Segall, M.H., and Dasen, P.R. (1992). Cross-cultural psychology: Research and applications. Cambridge, UK: Cambridge University Press.

Beyer, J. (1999). Two approaches to studying charismatic leadership: Competing or complimentary? Leadership Quarterly, (10) 2, 575-588.

Bollen, K.A. (1989). Structural equations with latent variables. John Wiley \& Sons, Inc.

Bond, M.H. Ed. (1995). Handbook of Chinese psychology. Hong Kong: Oxford University Press.

Bond, M.H. and Hwang, K.K. (1986). The social psychology of the Chinese people. Hong Kong: Oxford University Press

Bond, M.H. and Smith P.B. (1996). Cross-cultural social and organizational psychology. In Spence, J., Darley J.M. \& Donald J. Foss, Eds., Annual Review of Psychology, 47, 205-235. Palo Alto: Annual Reviews Inc.

Boomsma, A. (1982). The robustness of LISREL against small sample sizes in factor analysis models. In K. G. Joreskog and H. Wold, eds., Systems under Indirect Observation, Part I. Amsterdam: North-Holland, 149-173.

Bottger, P.C., Hallein, I.H., and Yetton, P.W. (1985). A cross-national study of leadership: Participation as a function of problem structure and leader power. Journal of Management Studies, 22: 358-368.

Bowers, D.G. and Seashore, S.E. (1967). Peer leadership within work groups. Personnel Administration, 30, 45-50.

Brown, A.F. (1967). Reactions to leadership. Educational Administrative Quarterly, 3 , 62-73.

Brown, E.D. and Sechrest, L. (1990). Experiments in cross-cultural research. Handbook of Cross-Cultural Psychology. Boston, MA: Allyn Bacon.

Bryman, A. (1993). Charismatic leadership in business organizations: some neglected issues. Leadership Quarterly, 4(3/4): 289-304. 
Burns, J.M. (1978). Leadership. New York: Harper and Row.

Byrne, B.M., Shavelson, R.J. and Muthen, B. (1989). Testing for the equivalence of factor covariance and mean structures: The issue of partial measurement invariance. Applied Psychological Measurement, 14, 183-196.

Chemers, M.M. (1993). An Integrative theory of leadership. In M.M. Chemers nd R. Ayman (Eds.), Leadership theory and research: Perspectives and directions. San Diego: Academic Press.

Chemers, M.M. (1997). An integrative theory of leadership. Mahwah, New Jersey: Lawrence Earlbaum Associates, Inc. Publishers.

Chemers, M.M., Ayman, R. and Fiedler, F. E. (1998). The contingency model of leadership effectiveness: Back to the future. In Dansereau, F. \& Yammarino, F. J. (Eds.), Leadership: The Multiple-Level Approaches, Classical and New Wave, 24, Part A, 135-144. Stamford, CT: JAI Press Inc.

Chen, M. (1995). Asian management systems: Chinese, Japanese and Korean Styles of Business. Thunderbird/Routledge Series in International Management, 213-228.

Cheung, G.W. and Rensvold, R. B. (2000). Assessing extreme and acquiescence response sets in cross-cultural research using structural equations modeling. Journal of Cross-Cultural Psychology, 31 (2), 187-212.

Cole, D.A. and Maxwell, S.E. (1985). Multitrait-multimethod comparisons across populations: A confirmatory factor analytic approach. Multivariate Behavioral Research, 20, 389-417.

Cole, D.A., Maxwell, S.E., Arvey, R. and Salas, E (1993). Multivariate group comparisons of variable systems: MANOVA and structural equation modeling. Psychological Bulletin, 114 (1), 174-184.

Conger, J.A. and Kanungo, R.A. (1987). Toward a behavioral theory of charismatic leadership in organizational settings. Academy of Management Review, 12; 637-647.

Conger, J.A. (1999). Charismatic and transformational leadership in organizations: An insider's perspective on these developing streams of research. Leadership Quarterly, 10 (2), 145-179.

Cunnningham, W., Cunningham, I.C.M., and Green, R.T. (1977). The ipsative process to reduce response set bias. Public Opinion Quarterly, 41 (Fall), 379-394. 
Dansereau, F., Graen, G.B., and Haga, W. (1975). A vertical dyad linkage approach to leadership in formal organizations. Organizational Behavior and Human Performance, 13: 46-78.

Davidson, A.R., Jaccard, J.J., Triandis, H.C., Morales, M.L., and Diaz-Guerro, R. (1976). Cross-cultural model testing: Toward a solution of the etic-emic dilemma. International Journal of Psychology, 11, 1-13.

Davis-Blake, A. and Pfeffer, J. (1988). Just a mirage: The search for dispositional effects in organizational research. Academy of Management Review, 14 (3), 385-400.

Davis, H.J., Ming, L.W., and Brosnan, T.F. (1986). The Farmer-Richman model: $A$ bibliographic essay emphasizing applicability to Singapore and Indonesia. Paper, Academy of Management, Chicago.

Den Hartog, D.N., House, R.J., Hanges, P.J. and Ruiz-Quintanilla, S. A. (1999). Culture specific and cross-culturally generalizable implicit leadership theories: Are attributes of charismatic/transformational leadership universally endorsed? Leadership Quarterly, 10 (2), 219-256.

Dorfman, P.W. and Howell, J.P. (1988). Dimensions of national culture and effective leadership patterns: Hofstede revisited. Advances in International comparative Management, 3, 127-150.

Dorfman, P.W. (1996). International and cross-cultural leadership. In Punnett, B.J. and Shenkar, O. (Eds.) Handbook for International Management Research. Blackwell Publishers Inc., 267-231.

Dorfman, P.W., Howell, J.P., Hibino, S., Lee, J.K., Tate, U. and Bautista, A. (1997). Leadership in Western and Asian countries: commonalities and differences in effective leadership processes across cultures. Leadership Quarterly, 8 (3), 233-274.

Drost, E.A. and Von Glinow, M.A. (1998). Leadership behavior in Mexico: Etic philosophies-emic practices. In Scandura, T.A. and Serapio, M.G. (Eds.), International Business and International Relations, 7, 3-29. Stamford, CT: JAI Press, Inc.

Douglas, S. P. and Craig, S. C. (1983). International Marketing Research. Englewood Cliffs, N.J.: Prentice Hall.

Evans, M.G. (1996). R.J. House's Path-Goal Theory of leader effectiveness. Leadership Quarterly, 7(3): 305-309.

Farmer, R.N. and Richman, B. (1965). Comparative Management \& Economic Progress. Homewood, Illinois: Richard D. Irwin \& Sons 
Farris, G.F. and Butterfield, D.A. (1972). Control theory in Brazilian organizations. Administrative Science Quarterly, 17 (4), 574-585.

Fiedler, F. E. (1967). A theory of leadership effectiveness. New York: McGraw Hill. Fiedler, F.E., Meuwse, W., and Oonk, S. (1961). An exploratory study of group creataivity in laboratory tasks. Acta Psychologica 18: 100-119.

Fiedler, F.E. and Chemers, M.M. (1974). Leadership and effective management. Glenview, IL: Scott, Foresman.

Fiedler, F.E. and Chemers, M.M. (1984). Improving leadership effectiveness: The leader-match concept. New York; Wiley.

Fisher, B.M. and Edwards, J.E. (1988). Consideration and Initiating structure and their relationships with leader effectiveness: A meta-analysis. Best Papers Proceedings, Academy of Management, Anaheim, CA, 201-205.

Fleishman, E.A. and Simmons, J. (1970). Relationship between leadership patterns and effectiveness ratings among Israeli foremen. Personnel psychology, 23, 169-172.

Fleishman, E.A. (1973). Twenty years of consideration and structure. In Fleishman E.A. and Hunt, J.G. (Eds.), Current developments in the study of leadership. Carbondale: Southern Illinois University Press.

Foster, G. (1960). Culture and Conquest: America's Spanish heritage (p. 4). Chicago: Quadrangle Books.

Fleishman, E.A. (1989). Examiner's manual for the Supervisory Behavior Description Opinion Questionnaire (Revised). Chicago: Science Research Associates.

Fleishman, E. A. (1998). Consideration and initiation of structure: another look at their role in leadership research. In F. Dansereau and F. J. Yammarino (Eds.), Leadership: The multiple-level approaches, Part A, 51-61.

Fleishman, E.A. and Simmons, J. (1970). Relationship between leadership patterns and effectiveness ratings among Israeli foremen. Personnel Psychology, 23, 169-172.

French, J.R.P. and Raven, B. (1959). The bases of social power. In D. Cartwright (Ed.), Studies in social power. Ann Arbor: University of Michigan, Institute for Social Research.

Gioia, D.A. and Sims, H.P. (1985). On avoiding the influence of implicit leadership theories in leader behavior descriptions. Educational and Psychological Measurement, 45, 217-232. 
Graen, G. and Cashman, J.F. (1975). A role-making model of leadership in formal organizations: A developmental approach. In J.G. Hunt and L.L. Larson (Eds.), Leadership frontiers, 143-165. Kent, OH: Kent State University Press.

Graen, G. and Scandura, T. (1987). Toward a psychology of dyadic organizing. Research in Organizational Behavior, 9, 175-208.

Graham, W.K. (1968). Description of leader behavior and evaluation of leaders as a function of LPC. Personnel psychology, 21, 457-464.

Gutmann, M.C. (1996). The meanings of macho: Being a man in Mexico City. Berkeley: University of California Press, 23.

Halpin, A.W. and Winer, B.J. (1957). A factorial study of the leader behavior descriptions. In R.M. Stogdill \& A.E. Coons (Eds.), Leader behavior: Its description and measurement. Columbus: Ohio State University, Bureau of Business Research.

Harbison, F. and Myers, C. (1959) Management in the industrial world: An international study. New York: NY: McGraw Hill.

Harrison, L.E. (1985). Underdevelopment is a state of mind: The Latin American case. The Center for International Affairs, Harvard University and University Press of America,132-150.

Heller, F.A. (1969). The role of business management in relation to economic development. International Journal of Comparative Sociology, 10, 292-298.

Heller, F.A. and B. Wilpert (1981). Competence and power in managerial decisionmaking: A study of senior-levels of organization in Eight Countries. London: Wiley.

Heller, F.,Drenth, P., Koopman, P., and Rus, V. (1988). Decisions in organizations. London: Sage Publications.

Hempdill, J.K. and Coons, A.E. (1957). Development of the leader behavior description questionnaire. In R.M. Stogdill and A. E. Coons (Eds.), Leader behavior: Its description and measurement. Columbus: Ohio State University, Bureau of Business Research.

Hofstede, G. (1980). Culture's Consequences: International differences in work-related values. London: Sage.

Hofstede, G. (1980a). Motivation, leadership, and organization: Do American theories apply abroad? Organizational Dynamics, 9, 42-63.

Hofstede, G. (1991). Cultures and organizations: Software of the mind. Berkshire, England: McGraw-Hill Book Company Europe. 
House, R.J. (1971). A path-goal theory of leader effectiveness. Administrative Science Quarterly, 16, 556-571.

Howell, J. P., Dorfman, P.W., Hibino, S., Lee, J.K., and Tate, U. (1994). Leadership in Western and Asian countries: Commonalities and differences in effective leadership processes and substitutes across cultures. Center for Business Research. New Mexico State University.

House, R. J. (1977). A 1976 theory of charismatic-leadership. In J.G. Hunt and L.L. Larson (Eds.), Leadership: The cutting edge, 189-207. Carbondale, IL: Southern Illinois University Press.

House, R.J., Shane, S, and Herold, D. (1996). Rumors of the death of dispositional theory and research in organizational behavior are greatly exaggerated. Academy of Management Review, 21(1), 203-224.

House R.J. and Aditya, R. N. (1997). The Social Scientific Study of Leadership: Quo Vaids? Journal of Management, 23 (3), 409-473.

House R.J. and Baetz, M.L. (1979). Leadership: Some empirical generalizations and new research directions. In B. Staw (Ed.), Research in Organizational Behavior, 1, 341 423. Greenwich, CT: JAI Press.

House, R.J. and Dessler, G. (1974). The path-goal theory of leadership: Some post hoc and a priori tests. In J.G. Hunt (ed), Contingency Approaches to Leadership.

Carbondale, IL: Southern Illinois University Press.

House, R.J. and Mitchell, T.R. (1974). Path-goal theory of leadership. Journal of Contemporary Business, 3; 81-97.

House, R.J. and Shamir, B. (1993). Toward the integration of transactional, charismatic and visionary theories. Leadership Theory and Research, 81-107. San Diego: Academic Press.

Hope Pelled, L. and Hill, K.D. (1997). Participative management in Northern Mexico: a study of maquiladoras. The International Journal of Human Resource Management, 8 (3), 197-212.

Howell, J.M., Dorfman, P.W., Hibino, S., Lee, J.K., and Tate, U. (1994). Leadership in Western and Asian countries: Commonalities and differences in effective leadership processes and substitutes across cultures. Center for Business Research. New Mexico State University.

Hsu, F.L.K. (1982). American and Chinese: Passage to differences. Honolulu: University of Hawaii Press. 
Hunt, J.G., Osborn, R.N., and Schriesheim, C.A. (1977). Some neglected aspects of leadership research. Proceedings, Midwest Academy of Management Meetings, Bloomington, IN, 364-375.

Hunt, J.G. and Peterson, M.F. (1997). International and cross-cultural leadership research. Leadership Quarterly, 8(3), 203-231.

Inkeles, A. (1966). The modernization of man. In M.Weiner (Ed.), Modernization. New York: Basic Books.

Jago, G., Reber, G., Bohnisch, W., Maczynski, J., Zavrel, J. and Dudorkin, J (1993). Culture' consequence? A seven-nation study of participation. Proceedings of the $24^{\text {th }}$ Annual Meeting of the Decision Sciences Institute. Washington, D.C.: Decision Sciences Institute.

Joreskog, K.G. (1981). Analysis of covariance structures. Scandanavian Journal of Statistics, 8, 65-92.

Joreskog, K.G. and Sorbom, D. (1993). LISREL8: Structural equation modeling with the SIMP LIS command language. Chicago: Scientific Software International.

Kahn, R.L. and Katz, D. (1951). Leadership practices in relation to productivity and morale. In D. Cargwright and A. Zander (Eds.), Group Dynamics, New York: Harper \& Row.

Katerberg, R. and Hom, P.W. (1981). Effects of within-group and between-groups variation in leadership. Journal of Applied Psychology, 66, 218-233.

Kavanagh, M.J. (1975). Expected supervisory behavior, interpersonal trust and environmental preferences. Some relationships based on a dyadic model of leadership. Organizational Behavior and Human Performance, 13, 17-30.

Kelloway, E.K. (1998). Using LISREL for structural equation modeling: A researchers guide. Thousands Oaks, CA: Sage Publications, Inc.

Kenis, I. (1977). A cross-cultural study of personality and leadership. Group and Organization Studies, 2, 49-50.

Kenny, D.A. and Zaccaro, S.J. (1983). An estimate of the variance due to traits in leadership. Journal of Applied Psychology, 68(4): 678-685

Korman, A.K. (1966). Consideration, initiating structure, and organizational criteria. Personnel Psychology, 18, 349-360. 
Kotabe, M. and Murray, J. Y. (1990). Linking product and process innovation and modes of international sourcing in global competition: A case of foreign multinational firms. Journal of International Business Studies, 21(3), 383-425.

Kouzes,, J.M. and Posner, B.Z. (1987). The leadership challenge: how to get extraordinary things done. San Francisco: Jossey-Bass.

Little, T.D. (1997) Mean and covariance structures (MACS) analyses of cross-cultural data: Practical and theoretical issues. Multivariate Behavioral Research, 32 (1), 53-76.

Little, T.D. (2000). On the comparability of constructs in cross-cultural research. A critique of Cheung and Rensvold. Journal of Cross Cultural Psychology, 31 (2), 213-219.

Lord, R.G., DeVader, C. L., and Alliger, G. M. (1986). A meta-analysis of the relation between personality traits and leadership perceptions: An application of validity generalization procedures. Journal of Applied Psychology, 71, 402-410.

Lord, R.G., Foti, R.J., and DeVader, C. (1984). A test of leadership categorization theory: Internal structure, information processing, and leadership perceptions. Organizational Behavior and Human Performance, 34, 343-378.

Lytle, A.L., Brett, J. M., Barsness, Z.I., Tinsley, C H., and Janssens, M. (1995). A paradigm for confirmatory cross-cultural research in organizational behavior, Research in Organizational Behavior, 17, 167-214. JAI Press Inc.

Marcoulides, G. A. \& Yavas, B. F. (1998). Examining leadership styles in different countries: An attempt to reconcile the culturalist and rationalist approaches to international management. Working Paper. California State University, Fullerton.

McClelland, D.C. (1961). The achieving society. New York: Van Nostrand Reinhold.

McClelland, D.C. (1975). Power: The inner experience. N.Y.: Irvington.

McClelland, D.C. (1985). Human motivation. Glenview, IL: Scott, Foresman.

McGraw, B. and Joreskog, K.G. (1971). Factorial invariance of ability measures in groups differing in intelligence and socioeconomic status. British Journal of Mathematical and Statistical Psychology, 24, 154-168.

Meredith, W. (1993). Measurement invariance, factor analysis and factorial invariance. Psychometrika, 58, 525-543.

Meade, R.D. (1967). An experimental study of leadership in India. Journal of Social Psychology, 72, 35-43. 
Meade, R.D. and Whittaker, J.D. (1967). A cross-cultural study of authoritarianism. Journal of Social Psychology, 72-349-364.

Medsker, G.J., Williams, L.J. and Holahan, P.J. (1994). A Review of current practices for evaluating causal models in organizational behavior and human resource management research. Journal of Management, 20 (2), 439-464.

Miller, J.A. (1973). A hierarchical structure of leadership behaviors (Tech. Rep. No. 66). Rochester, NY: University of Rochester, Management Research Center.

Miner, J.B. (1973). The management process: Theory, research and practice. New York; Macmillan.

Misumi, J. (1974). Action research on the development of leadership, decision-making processes and organizational performance in a Japanese shipyard. Paper, International congress of Applied Psychology, Liege, Belgium.

Misumi, J. and Shirakasi, S. (1966). An experimental study of the effects of supervisory behavior on productivity and morale in a hierarchical organization. Human Relations, 19 (3), 297-307.

Misumi, J. (1985). The behavioral science of leadership: An interdisciplinary Japanese research program. Ann Arbor, MI: University of Michigan Press.

Misumi, J. and Peterson, M.F. (1985). The performance-maintenance (PM) theory of leadership: Review of a Japanese research program. Administrative Science Quarterly, 30, 196-223.

Misumi, J. and Peterson, M.F. (1987). Supervision and leadership. Advances in organizational psychology: An international review. Newbury Park, CA: Sage Publications.

Mulaik, S. A. (1972). Foundations of factor analysis. New York: McGraw-Hill.

Mulaik, S.A., James, L.R., Van Alstine, J., Bennett, N., Lind, S. and Stillwell, C.D. (1989). An evaluation of goodness-of-fit indices for structural equation models. Psychological Bulletin, 105: 430-445.

Mullen, M. R. (1995). Diagnosing measurement equivalence in cross-national research, Journal of International Business Studies, 26 (3) , 573-596.

Muthen, B. and Kaplan, D. (1985). A comparison of some methodologies for the factor analysis of non-normal Likert variables. British Journal of Mathematical and Statistical Psychology, 38:171-189. 
Nunnally, J. C. (1978). Psychometric Theory. Second Edition. McGraw-Hill Book Company, p. 435.

Pelled L.H. and Hill, K.D. (1997). Participative management in Northern Mexico: a study of maquiladoras. The International Journal of Human Resource Management, 8 (3), 197-212.

Peters, L.H., Hartke, D.D. and Pohlman, J.T. (1985). Fiedler's contingency model of leadership: An application of the meta-analysis procedure of Schmidt and Hunter. Psychological Bulletin, 97: 274-285.

Podsakoff, P.M. and Schriesheim, C.A. (1985). Leader reward and punishment behavior: a methodological and substantive review. In B. Staw and L.L. Cummings (Eds.), Research in Organizational Behavior, San Francisco: Jossey-Bass.

Poortinga, Y. H. (1989). Equivalence of cross-cultural data: An overview of basic issues. International Journal of Psychology, 24, 737-756.

Redding, S.G. and Casey, T.W. (1976). Managerial beliefs among Asian managaers. In R. L. Taylor, M.J. O' Connell, R. A. Zawacki, and D. D. Warwick (Eds.), Proceedings of the Academy of Management $36^{\text {th }}$ Annual Meeting, 351-356. Academy of Management, 1976 Annual Meeting in Kansas City, Kansas.

Rim, Y. (1965). Leadership attitudes and decisions involving risk. Personnel Psychology, 18, 423-430.

Riordan, C.M. and Vandenberg, R.J. (1994). A central question in cross-cultural research: Do employees of different cultures interpret work-related measures in an equivalent manner? Journal of Management, 20, 643-671.

Rush, M.C., Thomas, J.C. and Lord, R.G. (1977). Implicit leadership theory: A potential threat to the internal validity of leader behavior questionnaires. Organizational Behavior and Human Performance, 20, 93-110.

Scandura, Von Glinow, M.A. and Lowe, K. (1999). When East meets West: Leadership "Best Practices" in the United States and Middle East. In Gessner, M.J. and Arnold, V. (Eds.), Advances in Global Leadership, Volume 1. Stamford, CT: JAI Press

Shamir, B. and Howell, J. M. (1999). Organizational and contextual influences on the emergence and effectiveness of charismatic leadership. Leadership Quarterly, 19(20), 257-283.

Schneider, B. (1983). International psychology \& organizational behavior. In L. L. Cummings \& B.M. Shaw (Eds.) Research in Organizational behavior, 5, 1-31.

CT: JAI Press. 
Schrieheim, C.A. and Stogdill, R.M. (1975). Differences in factor structure across three versions of The Ohio State leadership scales. Personnel psychology, 29, 189-206.

Schriesheim, C.A., House, R.J. and Kerr, S. (1976). Leader initiating structure: A reconciliation of discrepant research results and some empirical tests. Organizational Behavior and Human Performance, 15, 297-321.

Schriesheim, C.A. and Kerr, S. (1974). Psychometric properties of the Ohio State leadership scales. Psychological Bulletin, 81, 756-765.

Schriesheim, C. A. and Kerr, S. (1977). Theories and measures of leadership: A critical appraisal of present and future directions. In J.C. Hunt and L.L. Larson (Eds.), Leadership: The cutting edge. Carbondale, IL: Southern Illinois University Press.

Schriesheim, C. A and Von Glinow, M.A. (1977). The path goal theory of leadership: A theoretical and empirical analysis. Academy of Management Journal, 20, 398-405.

Schriesheim, C.A., Kinicki, A.J. and Schriesheim, J.F. (1979). The effect of leniency of leader behavior descriptions. Organizational Behavior and Human Performance, 23, 1-29.

Schriesheim, C. and Neider, L.L. (1996). Path-goal leadership theory: The long and winding road. The Leadership Quarterly, 7(3): 317-321.

Schriesheim, C. A., Cogliser, C.C. and Neider, L.L. (1998). Is it "trustworthy"? A Multiple-levels-of-analysis reexamination of an Ohio State leadership Study, with implications for research. In F. Dansereau and F. J. Yammarino (Eds.), Leadership: The multiple-level approaches, Part A, 3-43..

Seltzer, J. and Bass, B.M. (1987). Leadership is more than initiation of structure and consideration. Paper presented at the American Psychological Association, New York.

Shaw, M. and Costanzo, P.R. (1982). Theories of social psychology. $2^{\text {nd }}$ edition. New York: McGraw-Hill.

Singh, S.N. and Arya, H.P. (1965). Value-orientations of local village leaders. Manas, 12, 145-156.

Sinha, J.B.P. (1980). The nurturant task leader. New Delhi: Concept.

Sirota, D. and Greenwood, J.M. (1971). Understand your overseas work force. Harvard Business Review, 49 (1), 53-60. 
Smith, P.B., Dugan, S., and Trompenaars, F. (1996). National culture and the values of organizational employees: a dimensional analysis across 43 nations. Journal of CrossCultural Psychology, 27 (2): 231-264.

Smith, P.B. and Peterson, M.F. (1988). Leadership, organizations and culture: An event management model. London: Sage Publications.

Smith, P.B., Misumi, J., Tayeb, M., Peterson, M., and Bond, M. (1989). On the generality of leadership style measures across cultures. Journal of Occupational Psychology, 62 (2), 97-109.

Smith, P.C., Kendall, L.M. and Hulin, C.L. (1969). The measurement of satisfaction in work and retirement. Chicago: Rand McNally.

Steers, R.M., Shin, Y.K., and Ungson, G.R. (1989). The Chaebol: Korea's new industrial might. New York: Harper.

Stogdill, R.M. (1963). Manual for the leader behavior description questionnaire-form XII. Columbus: Ohio State University, Bureau of Business Research.

Stogdill, R.M., Goode, O.S. and Day, D.R. (1963). The leader behavior of corporation presidents. Personnel Psychology, 16, 127-132.

Stogdill, R.M., Goode, O.S. and Day, D.R. (1963). The leader behavior of United States senators. Journal of Psychology, 56, 3-8.

Stogdill, R.M., Goode, O.S. and Day, D.R. (1964). The leader behavior of presidents of labor unions. Personnel Psychology, 17, 49-57.

Stogdill, R.M., Goode, O.S. and Day, D.R. (1965). The leader behavior of university presidents. Columbus: Ohio State University, Bureau of Business Research.

Strube, M.J. and Garcia, J.E. (1983). On the proper interpretation of empirical findings: Strube and Garcia (1981) revisited. Psychological Bulletin, 93(3), 600-603.

Strube, M.J. and Garcia, J.E. (1981). A meta-analytical investigation of Fiedler's Contingency Model of leadership effectiveness. Psychological Bulletin, 90, 303-321.

Tannenbaum, A.S. and Schmidt, W.H. (1958). How to choose a leadership pattern. Harvard Business Review, 36(2), 95-101.

Taylor, J.C. and Bowers, D.G. (1972). Survey of organizations: Toward a machinescored standardized questionnaire instrument. Ann Harbor: University of Michigan, Institute for Social Research. 
Tichy, N.M. and Devanna, M. (1986). Transformational leadership. New York: Wiley.

Teagarden, M.B., Von Glinow, M.A., Bowen, D., Frayne, C., Nason, S., Huo, P., Milliman, J., Arias, M., Butler, M., Geringer, M., Kim, N., Scullion, H., Lowe, K., and Drost, E.A. (1995). Toward building a theory of comparative management research methodology: An idiographic case study of the best international human resources management project. Academy of Management Journal, 38, 1261-1287.

Teagarden, M.B. and Von Glinow, M.A. (1997). Human Resource Management in Cross-Cultural contexts: Emic Practices versus Etic Philosophies. Management International Review, 37, 7-20.

Triandis, H.C. (1993). The contingency model in cross-cultural perspective. In M.M. Chemers and Ayman, R. (Eds.), Leadership theory and research: Perspectives and directions, 167-188. San Diego: Academic Press.

Triandis, H.C. (1994). Cross-cultural industrial and organizational psychology. In H.C. Triandis et al. (Eds.), Handbook of industrial and organizational psychology $\left(2^{\text {nd }}\right)$, 4, 103-172. CA: Consulting Psychological Press.

Tscheulin, D. (1973). Leader behaviors in German industry. Journal of Applied Psychology, 57, 29-31.

Tucker. L.R. and Lewis, C. (1973). A reliability coefficient for maximum likelihood factor analysis. Psychosometrika, 38, 1-10.

Van de Vijver, F.J.R and Tanzer, N.K. (1997). Bias and Equivalence in Cross-Cultural Assessment: An Overview. European Review of Applied Psychology, 47 (4), 4, 263-279.

Van de Vijver, F.J.R. and Leung, K. (1997). Methods and data analysis for crosscultural research. SAGE Publications: Thousand Oaks, London, New Delhi.

Van de Vijver, F.J.R. and Leung, K. (1999). Methodological issues in psychological research on culture. Journal of Cross-Cultural Psychology, 31 (1), 33-51.

Vargas, G.A. and Johnson, T. W. (1993). An analysis of operational experience in the U.S./Mexico production sharing (maquiladora) program. Journal of Operations Management, 11, 17-34.

Vecchio, R.P. (1983). Assessing the validity of Fiedler's contingency model of leader effectiveness: a closer look at Strube and Garcia. Psychological Bulletin, 93(2), 404-408.

Vroom, V.H. and Yetton, P.W. (1973). Leadership and decision-making. Pittsburgh, PA: Universisty of Pittsburgh Press. 
Vroom, V.H. and Jago, A.G. (1988). The new leadership: Managing participation in organizations. Englewood cliffs, NJ: Prentice Hall.

Wahba, M. and Bridwell, L. (1975). Maslow reconsidered: A review of the need hierarchy theory. Organizational Behavior and Human Performance, 15 (2), 212-240.

Webber, R. H. (1969). Convergence or divergence. Columbia Journal of World Business, 4(3), 185-195.

Weissenger, P. and Kavanagh, M.J. (1972). The independence of initiation of structure and consideration: A review of the literature. Personnel Psychology, 25, 199-130.

West, S.G., Finch, J.F. and Curran, P.J. (1995). Structural equation models with nonnormal variables: Problems and remedies. In R.H. Hoyle (ed.) Structural equation modeling: concepts, issues and applications, 56-75. Newbury Park, CA: Sage Publications, Inc.

Wheaton, B. (1987). Assessment of fit in over-identified models with latent variables. Sociological Methods and Research, 16, 118-154.

Williams, L. J. (1995). Covariance structure modeling in organizational research: Problems with the method vs. applications of the method. Journal of Organizational Behavior, 16, 225-233.

Wofford, J.C. (1971). Managerial behaviors, situational factors, and productivity and morale. Administrative Science Quarterly, 16, 10-17.

Yammarino, F.J. and Bass, B.M. (1990). Long-term forecasting of transformational leadership and its effects among naval officers: Some preliminary findings. In K.E. Clark and Clark, M.B. (Eds.), Measures of leadership. Greensboro, NC: Center for Creative Leadership.

Yukl, G.A. (1968). Leader personality and situational variables as co-determinants of leader behavior. Dissertation Abstracts, 29, 406.

Yukl. G.A. (1971). Toward a behavioral theory of leadership. Organization Behavior and Human Performance, 6, 414-440.

Yukl, G. A. (1989). Leadership in organizations, (2 ${ }^{\text {ne }}$ ed.) Englewood Cliffs, NJ: Prentice Hall.

Yukl, G.A. (1999). An evaluation of conceptual weaknesses in transformational and charismatic leadership theories. Leadership Quarterly, 10(2), 285-305. 
Yukl, G.A. and Hunt, J.B. (1976). An empirical comparison of the Michigan four-factor and Ohio State LBDQ leadership scales. Organizational Behavior and Human Performance, 17, 45-65.

Yukl, G.A. and Van Fleet, D. (1992). Theory and research on leadership in organizations. Handbook of Industrial and Organizational Psychology. Palo alto, CA: Consulting Psychology Press, 147-197. 
APPENDIX 
APPENDIX: Percentage of Male and Female Managers: Total Sample, Managers from 12 Countries/Regions

\begin{tabular}{|c|c|c|c|c|c|}
\hline & & Frequency & Percent & Valid Percent & $\begin{array}{c}\text { Cumulative } \\
\text { Percent }\end{array}$ \\
\hline \multirow[t]{3}{*}{ Valid } & MALE & 1684 & 71.9 & 76.0 & 76.0 \\
\hline & FEMALE & 531 & 22.7 & 24.0 & 100.0 \\
\hline & Total & 2215 & 94.6 & 100.0 & \\
\hline \multirow{2}{*}{$\begin{array}{l}\text { Missing } \\
\text { Total }\end{array}$} & System & 126 & 5.4 & & \\
\hline & & 2341 & 100.0 & & \\
\hline
\end{tabular}

APPENDIX: Level of Education: Total Sample, Managers from 12 countries/regions

\begin{tabular}{|c|c|c|c|c|c|}
\hline & & Frequency & Percent & Valid Percent & $\begin{array}{c}\text { Cumulative } \\
\text { Percent } \\
\end{array}$ \\
\hline \multirow[t]{8}{*}{ Valid } & 1 & 1 & .0 & .0 & .0 \\
\hline & $\begin{array}{l}\text { HIGH SCHOOL OR } \\
\text { LOWER }\end{array}$ & 222 & 9.5 & 9.8 & 9.9 \\
\hline & $\begin{array}{l}\text { SOME COLLEGE } \\
\text { EDUCATION }\end{array}$ & 239 & 10.2 & 10.6 & 20.4 \\
\hline & BACHELOR'S DEGREE & 872 & 37.2 & 38.6 & 59.0 \\
\hline & $\begin{array}{l}\text { SOME GRADUATE } \\
\text { WORK }\end{array}$ & 459 & 19.6 & 20.3 & 79.3 \\
\hline & GRADUATE DEGREE & 337 & 14.4 & 14.9 & 94.2 \\
\hline & OTHER & 131 & 5.6 & 5.8 & 100.0 \\
\hline & Total & 2261 & 96.6 & 100.0 & \\
\hline Missing & System & 80 & 3.4 & & \\
\hline Total & & 2341 & 100.0 & & \\
\hline
\end{tabular}

APPENDIX: Age Categories: Total Sample, Managers from 12 Countries/Regions

\begin{tabular}{|ll|r|r|r|r|}
\hline & Frequency & Percent & Valid Percent & $\begin{array}{c}\text { Cumulative } \\
\text { Percent }\end{array}$ \\
\hline Valid & LESS THAN 30 & 290 & 12.4 & 12.5 & 12.5 \\
& $30-39$ & 867 & 37.0 & 37.3 & 49.7 \\
& $40-49$ & 865 & 37.0 & 37.2 & 86.9 \\
& $50-59$ & 287 & 12.3 & 12.3 & 99.2 \\
& 60 OR OLDER & 18 & .8 & .8 & 100.0 \\
& Total & 2327 & 99.4 & 100.0 & \\
Missing & System & 14 & .6 & & \\
Total & 2341 & 100.0 & & \\
\hline
\end{tabular}


APPENDIX: Salary Levels: Total Sample, Managers from 12 Countries/Regions

\begin{tabular}{|c|c|c|c|c|c|}
\hline & & Frequency & Percent & Valid Percent & $\begin{array}{c}\text { Cumulative } \\
\text { Percent }\end{array}$ \\
\hline \multirow[t]{6}{*}{ Valid } & LESS THAN $\$ 25,000$ & 446 & 19.1 & 20.0 & 20.0 \\
\hline & $\$ 25,000$ TO 50,000 & 442 & 18.9 & 19.8 & 39.8 \\
\hline & $\$ 50,001$ TO 75,000 & 548 & 23.4 & 24.6 & 64.4 \\
\hline & $\$ 75,001$ TO $\$ 100,000$ & 499 & 21.3 & 22.4 & 86.8 \\
\hline & $\begin{array}{l}\text { GREATER THAN } \\
\$ 100,000\end{array}$ & 294 & 12.6 & 13.2 & 100.0 \\
\hline & Total & 2229 & 95.2 & 100.0 & \\
\hline \multirow{2}{*}{$\begin{array}{l}\text { Missing } \\
\text { Total } \\
\end{array}$} & System & 112 & 4.8 & & \\
\hline & & 2341 & 100.0 & & \\
\hline
\end{tabular}

APPENDIX: Education Levels for Managers by Country/Region

Count

\begin{tabular}{|c|c|c|c|c|c|c|c|c|c|}
\hline & & \multicolumn{7}{|c|}{ EOUCATION } & \multirow[b]{2}{*}{ Total } \\
\hline & & 1 & $\begin{array}{c}\text { HIGH } \\
\text { SCHOOL } \\
\text { OR LOWER } \\
\end{array}$ & $\begin{array}{c}\text { SOME } \\
\text { COLLEGE } \\
\text { EDUCATION } \\
\end{array}$ & $\begin{array}{c}\text { BACHELOR'S } \\
\text { DEGREE }\end{array}$ & $\begin{array}{c}\text { SOME } \\
\text { GRADUATE } \\
\text { WORK }\end{array}$ & $\begin{array}{c}\text { GRADUATE } \\
\text { OEGREE } \\
\end{array}$ & OTHER & \\
\hline \multirow{13}{*}{$\begin{array}{l}\text { COUNTRY } \\
\text { CODE }\end{array}$} & USA & \multirow{12}{*}{1} & 1 & 7 & 18 & 53 & 58 & 4 & 141 \\
\hline & CANADA & & 6 & 15 & 4 & 18 & 28 & 6 & 117 \\
\hline & CHINA & & 47 & 15 & 78 & 1 & 3 & 37 & 181 \\
\hline & TAINAN & & 2 & 13 & 84 & 19 & & & 118 \\
\hline & JAPAN & & 58 & 26 & 158 & 1 & 26 & 4 & 273 \\
\hline & $\begin{array}{l}\text { MEXICO } \\
\text { LATIN }\end{array}$ & & 10 & 17 & 86 & 28 & 22 & 14 & 178 \\
\hline & $\begin{array}{l}\text { LATIN } \\
\text { AMERICA }\end{array}$ & & 1 & 22 & 64 & 24 & 25 & 3 & 139 \\
\hline & INDONESIA & & 7 & 11 & 13 & 149 & 5 & 47 & 232 \\
\hline & AUSTRALIA & & 41 & 49 & 121 & 82 & 96 & & 389 \\
\hline & PHILIPPINES & & & 1 & 52 & 66 & 44 & 1 & 164 \\
\hline & KOREA & & 34 & 33 & 144 & 2 & 15 & 3 & 231 \\
\hline & GULF & & 15 & 30 & 10 & 16 & 15 & 12 & 98 \\
\hline & & 1 & 222 & 239 & 872 & 459 & 337 & 131 & 2261 \\
\hline
\end{tabular}


APPENDIX: Percentage of Male and Female Managers by Country/Region

\begin{tabular}{|c|c|c|c|c|c|}
\hline & & & \multicolumn{2}{|c|}{ GENDER } & \multirow[b]{2}{*}{ Total } \\
\hline \multirow{49}{*}{$\begin{array}{l}\text { COUNTRY } \\
\text { CODE }\end{array}$} & & & MALE & FEMALE & \\
\hline & USA & Count & 91 & 48 & 139 \\
\hline & & $\begin{array}{l}\text { \% within } \\
\text { COUNTRY CODE }\end{array}$ & $65.5 \%$ & $34.5 \%$ & $100,0 \%$ \\
\hline & & \% within GENDER & $5.4 \%$ & $9.0 \%$ & $6.3 \%$ \\
\hline & & $\%$ of Total & $4.1 \%$ & $2.2 \%$ & $6.3 \%$ \\
\hline & CANADA & Count & 95 & 21 & 116 \\
\hline & & $\begin{array}{l}\text { \% within } \\
\text { COUNTRY COOE }\end{array}$ & $81.9 \%$ & $18.1 \%$ & $100.0 \%$ \\
\hline & & $\%$ within GENDER & $5.6 \%$ & $4.0 \%$ & $5.2 \%$ \\
\hline & & $\%$ of Total & $4.3 \%$ & $.9 \%$ & $5.2 \%$ \\
\hline & CHINA & Count & 123 & 61 & 184 \\
\hline & & $\begin{array}{l}\text { \% within } \\
\text { COUNTRY CODE }\end{array}$ & $66.8 \%$ & $33.2 \%$ & $100.0 \%$ \\
\hline & & \% within GENDER & $7.3 \%$ & $11.5 \%$ & $8.3 \%$ \\
\hline & & $\%$ of Total & $5.6 \%$ & $2.8 \%$ & $8.3 \%$ \\
\hline & TAWAN & Count & 101 & 16 & 117 \\
\hline & & $\begin{array}{l}\% \text { within } \\
\text { COUNTRY CODE }\end{array}$ & $86.3 \%$ & $13.7 \%$ & $100.0 \%$ \\
\hline & & $\%$ within GENDER & $6.0 \%$ & $3.0 \%$ & $5.3 \%$ \\
\hline & & $\%$ of Total & $4.6 \%$ & $.7 \%$ & $5.3 \%$ \\
\hline & JAPAN & Count & 247 & 19 & 266 \\
\hline & & $\begin{array}{l}\text { \% within } \\
\text { COUNTRY CODE }\end{array}$ & $92.9 \%$ & $7.1 \%$ & $100.0 \%$ \\
\hline & & $\%$ within GENDER & $14.7 \%$ & $3.6 \%$ & $12.0 \%$ \\
\hline & & $\%$ of Total & $11.2 \%$ & $9 \%$ & $12.0 \%$ \\
\hline & MEXICO & Count & 121 & 30 & 151 \\
\hline & & $\begin{array}{l}\% \text { within } \\
\text { COUNTRY CODE }\end{array}$ & $80.1 \%$ & $19.9 \%$ & $100.0 \%$ \\
\hline & & $\%$ within GENDER & $7.2 \%$ & $5.6 \%$ & $6.8 \%$ \\
\hline & & $\%$ of Total & $5.5 \%$ & $1.4 \%$ & $6.8 \%$ \\
\hline & LATIN & Count & 80 & 51 & 131 \\
\hline & & $\begin{array}{l}\text { \% within } \\
\text { COUNTRY CODE }\end{array}$ & $61.1 \%$ & $38.9 \%$ & $100.0 \%$ \\
\hline & & $\%$ within GENDER & $4.8 \%$ & $9.6 \%$ & $5.9 \%$ \\
\hline & & $\%$ of Total & $3.6 \%$ & $2.3 \%$ & $5.9 \%$ \\
\hline & INDONESIA & Count & 195 & 43 & 238 \\
\hline & & $\begin{array}{l}\text { \% within } \\
\text { COUNTRY CODE }\end{array}$ & $81.9 \%$ & $18.1 \%$ & $100.0 \%$ \\
\hline & & $\%$ within GENDER & $11.6 \%$ & $8.1 \%$ & $10.7 \%$ \\
\hline & & $\%$ of Total & $8.8 \%$ & $1.9 \%$ & $10.7 \%$ \\
\hline & AUSTRALIA & Count & 268 & 138 & 406 \\
\hline & & $\begin{array}{l}\text { \% within } \\
\text { COUNTRY CODE }\end{array}$ & $66.0 \%$ & $34.0 \%$ & $100.0 \%$ \\
\hline & & \% within GENOER & $15,9 \%$ & $26.0 \%$ & $18.3 \%$ \\
\hline & & $\%$ of Total & $12.1 \%$ & $6.2 \%$ & $18.3 \%$ \\
\hline & PHILIPPINES & Count & 58 & 80 & 138 \\
\hline & & $\begin{array}{l}\% \text { within } \\
\text { COUNTRY CODE }\end{array}$ & $42.0 \%$ & $58.0 \%$ & $100.0 \%$ \\
\hline & & $\%$ within GENDER & $3.4 \%$ & $15.1 \%$ & $6.2 \%$ \\
\hline & & $\%$ of Total & $2.6 \%$ & $3.6 \%$ & $6.2 \%$ \\
\hline & KOREA & Count & 227 & 3 & 230 \\
\hline & & $\begin{array}{l}\text { \% within } \\
\text { COUNTRY COOE }\end{array}$ & $98.7 \%$ & $1.3 \%$ & $100,0 \%$ \\
\hline & & $\%$ within GENDER & $13.5 \%$ & $.6 \%$ & $10.4 \%$ \\
\hline & & $\%$ of Total & $10.2 \%$ & $.1 \%$ & $10.4 \%$ \\
\hline & GULF & Count & 78 & 21 & 99 \\
\hline & & $\begin{array}{l}\text { \% within } \\
\text { COUNTRY CODE }\end{array}$ & $78.8 \%$ & $21.2 \%$ & $100.0 \%$ \\
\hline & & \% within GENDER & $4.6 \%$ & $4.0 \%$ & $4.5 \%$ \\
\hline & & $\%$ of Total & $3.5 \%$ & $.9 \%$ & $4.5 \%$ \\
\hline \multirow[t]{4}{*}{ Total } & & Count & 1684 & 531 & 2215 \\
\hline & & $\begin{array}{l}\text { \% within } \\
\text { COUNTRY CODE }\end{array}$ & $76.0 \%$ & $24.0 \%$ & $100.0 \%$ \\
\hline & & $\%$ within GENDER & $100.0 \%$ & $100,0 \%$ & $100.0 \%$ \\
\hline & & $\%$ of Totgl & $76.0 \%$ & $24.0 \%$ & $100.0 \%$ \\
\hline
\end{tabular}


APPENDIX: Educational Level for Mate and Femate Managers by Country/Region

\begin{tabular}{|c|c|c|c|c|c|c|c|c|c|c|}
\hline \multirow[b]{2}{*}{ GENDER } & & & & \multicolumn{6}{|c|}{ EDUCATION } & \multirow[b]{2}{*}{ Total } \\
\hline & & & & $\begin{array}{c}\text { HIGH } \\
\text { SCHOOL } \\
\text { ORLOWER } \\
\end{array}$ & $\begin{array}{l}\text { SOME } \\
\text { COLLEGE } \\
\text { EDUCATION }\end{array}$ & $\begin{array}{c}\text { BACHELOR'S } \\
\text { DEGREE }\end{array}$ & $\begin{array}{c}\text { SOME } \\
\text { GRADUATE } \\
\text { WORK } \\
\end{array}$ & $\begin{array}{l}\text { GRAQUATE } \\
\text { DEGREE }\end{array}$ & OTHER & \\
\hline \multirow[t]{52}{*}{ MALE } & \multirow{48}{*}{$\begin{array}{l}\text { COUNTRY } \\
\text { CODE }\end{array}$} & USA & Count & 1 & 3 & 9 & 33 & 42 & 3 & 91 \\
\hline & & & $\begin{array}{l}\text { \% within COUNTRY } \\
\text { CODE }\end{array}$ & $1.1 \%$ & $3.3 \%$ & $9.9 \%$ & $36.3 \%$ & $46.2 \%$ & $3.3 \%$ & $1000 \%$ \\
\hline & & & \% within EDUCATION & $6 \%$ & $1.8 \%$ & $1.4 \%$ & $10.4 \%$ & $17.6 \%$ & $3.1 \%$ & $5.5 \%$ \\
\hline & & & $\%$ of Total & $.1 \%$ & $.2 \%$ & $.5 \%$ & $2.0 \%$ & $2.6 \%$ & $.2 \%$ & $5.5 \%$ \\
\hline & & CANADA & Count & 3 & 8 & 39 & 16 & 24 & 5 & 95 \\
\hline & & & $\begin{array}{l}\text { \% within COUNTRY } \\
\text { COOE }\end{array}$ & $32 \%$ & $8.4 \%$ & $41.1 \%$ & $168 \%$ & $253 \%$ & $5.3 \%$ & $100.0 \%$ \\
\hline & & & \% within EDUCATION & $1.7 \%$ & $4.8 \%$ & $6.0 \%$ & $50 \%$ & $10.0 \%$ & $5.2 \%$ & $5.8 \%$ \\
\hline & & & $\%$ of Total & $2 \%$ & $.5 \%$ & $2.4 \%$ & $10 \%$ & $15 \%$ & $3 \%$ & $5.8 \%$ \\
\hline & & CHINA & Count & 29 & 12 & $\$ 1$ & 1 & 3 & 22 & 118 \\
\hline & & & $\begin{array}{l}\text { \% within COUNTRY } \\
\text { CODE }\end{array}$ & $24,6 \%$ & $10.2 \%$ & $43.2 \%$ & $.3 \%$ & $2.5 \%$ & $18.6 \%$ & $100.0 \%$ \\
\hline & & & $\%$ within EDUCATION & $168 \%$ & $7,2 \%$ & $7.8 \%$ & $.3 \%$ & $13 \%$ & $22.9 \%$ & $7.2 \%$ \\
\hline & & & $\%$ of Total & $1.8 \%$ & $.7 \%$ & $3.1 \%$ & $.1 \%$ & $2 \%$ & $1.3 \%$ & $7.2 \%$ \\
\hline & & TANAN & Count & 2 & 10 & 73 & 16 & & & 101 \\
\hline & & & $\begin{array}{l}\text { \% within COUNTRY } \\
\text { COOE }\end{array}$ & $2.0 \%$ & $9.9 \%$ & $n 2.3 \%$ & $15.8 \%$ & & & $100.0 \%$ \\
\hline & & & \% within EDUCATION & $1.2 \%$ & $60 \%$ & $11.2 \%$ & $5.0 \%$ & & & $6.1 \%$ \\
\hline & & & \% of Total & $.1 \%$ & $.6 \%$ & $4.4 \%$ & $1.0 \%$ & & & $6.1 \%$ \\
\hline & & JAPAN & Count & 55 & 22 & 139 & 1 & 25 & 4 & 246 \\
\hline & & & $\begin{array}{l}\text { \% within COUNTRY } \\
\text { CODE }\end{array}$ & $22.4 \%$ & $8.9 \%$ & $56.5 \%$ & $.8 \%$ & $10,2 \%$ & $1.6 \%$ & $100.0 \%$ \\
\hline & & & \% within EDUCATION & $31.8 \%$ & $13.2 \%$ & $21.3 \%$ & $.3 \%$ & $10.5 \%$ & $4.2 \%$ & $14.9 \%$ \\
\hline & & & $\%$ of Total & $3.3 \%$ & $1.3 \%$ & $8.4 \%$ & $1 \%$ & $1.5 \%$ & $.2 \%$ & $14.9 \%$ \\
\hline & & MEXICO & Count & 8 & II & 55 & 18 & 17 & 9 & 118 \\
\hline & & & $\begin{array}{l}\text { \% within COUNTRY } \\
\text { CODE }\end{array}$ & $6.8 \%$ & $93 \%$ & $46.6 \%$ & $15.3 \%$ & $14.4 \%$ & $7.6 \%$ & $100,0 \%$ \\
\hline & & & $\%$ within EDUCATION & $4.6 \%$ & $6.6 \%$ & $8.4 \%$ & $5.7 \%$ & $7.1 \%$ & $9.4 \%$ & $7.2 \%$ \\
\hline & & & $\%$ of Total & $.5 \%$ & $.7 \%$ & $3,3 \%$ & $1.1 \%$ & $10 \%$ & $.5 \%$ & $7.2 \%$ \\
\hline & & LATIN AMERICA & Count & 1 & 8 & 35 & 13 & 20 & 3 & 80 \\
\hline & & & $\begin{array}{l}\text { \% within COUNTRY } \\
\text { COOE }\end{array}$ & $1.3 \%$ & $10.0 \%$ & $43.8 \%$ & $16.3 \%$ & $25.0 \%$ & $3.8 \%$ & $100.0 \%$ \\
\hline & & & \% within EDUCATION & $6 \%$ & $4.8 \%$ & $5.4 \%$ & $4.1 \%$ & $8.4 \%$ & $3.1 \%$ & $49 \%$ \\
\hline & & & $\%$ of Total & $.1 \%$ & $.5 \%$ & $2.1 \%$ & $.8 \%$ & $12 \%$ & $2 \%$ & $49 \%$ \\
\hline & & INDONESIA & Count & 4 & 11 & 10 & 125 & 4 & 38 & 192 \\
\hline & & & $\begin{array}{l}\text { \% within COUNTRY } \\
\text { CODE }\end{array}$ & $2.1 \%$ & $5.7 \%$ & $5.2 \%$ & $65.1 \%$ & $2.1 \%$ & $19.8 \%$ & $100.0 \%$ \\
\hline & & & \% within EDUCATION & $2.3 \%$ & $6.6 \%$ & $1.5 \%$ & $39.3 \%$ & $1.7 \%$ & $39.6 \%$ & $11.7 \%$ \\
\hline & & & \% of Total & $2 \%$ & $.7 \%$ & $.6 \%$ & $7.6 \%$ & $2 \%$ & $2.3 \%$ & $11.7 \%$ \\
\hline & & AUSTRALIA & Count & 24 & 29 & 81 & 51 & 60 & & 245 \\
\hline & & & $\begin{array}{l}\text { \% within COUNTRY } \\
\text { CODE }\end{array}$ & $9.8 \%$ & $11.8 \%$ & $33.1 \%$ & $20.8 \%$ & $24.5 \%$ & & $100: 0 \%$ \\
\hline & & & $\%$ within EDUCATION & $13.9 \%$ & $17.4 \%$ & $12.4 \%$ & $160 \%$ & $25.1 \%$ & & $14.9 \%$ \\
\hline & & & $\%$ of Total & $1.5 \%$ & $1.8 \%$ & $49 \%$ & $3.1 \%$ & $3.6 \%$ & & $149 \%$ \\
\hline & & PHILIPPINES & Count & & & 14 & 27 & 17 & & 58 \\
\hline & & & $\begin{array}{l}\text { \% within COUNTRY } \\
\text { CODE }\end{array}$ & & & $24.1 \%$ & $46.6 \%$ & $29.3 \%$ & & $100,0 \%$ \\
\hline & & & \% within EDUCATION & & & $2.1 \%$ & $8.5 \%$ & $7.1 \%$ & & $3.5 \%$ \\
\hline & & & $\%$ of Total & & & $.9 \%$ & $1.6 \%$ & $10 \%$ & & $35 \%$ \\
\hline & & KOREA & Count & 34 & 31 & 1.42 & 2 & 14 & 3 & 226 \\
\hline & & & $\begin{array}{l}\text { \% within COUNTRY } \\
\text { CODE }\end{array}$ & $150 \%$ & $13.7 \%$ & $62.8 \%$ & $.9 \%$ & $6.2 \%$ & $1.3 \%$ & $100.0 \%$ \\
\hline & & & $\%$ within EDUCATION & $19.7 \%$ & $18.6 \%$ & $21.7 \%$ & $.6 \%$ & $5.9 \%$ & $3.1 \%$ & $13.7 \%$ \\
\hline & & & $\%$ of Total & $2.1 \%$ & $1,9 \%$ & $8.6 \%$ & $1 \%$ & $9 \%$ & $2 \%$ & $137 \%$ \\
\hline & & GULF & Count & 12 & 22 & 6 & 15 & 13 & 9 & 77 \\
\hline & & & $\begin{array}{l}\text { \% within COUNTRY } \\
\text { CODE }\end{array}$ & $15.6 \%$ & $28.6 \%$ & $78 \%$ & $19.5 \%$ & $16.9 \%$ & $11.7 \%$ & $100.0 \%$ \\
\hline & & & \% within EDUCATION & $69 \%$ & $13.2 \%$ & $9 \%$ & $4.7 \%$ & $5.4 \%$ & $9.4 \%$ & $4.7 \%$ \\
\hline & & & $\%$ of Total & $.7 \%$ & $1.3 \%$ & $4 \%$ & $.9 \%$ & $.8 \%$ & $5 \%$ & $47 \%$ \\
\hline & \multirow[t]{4}{*}{ Total } & & Count & 173 & 167 & 654 & 318 & 239 & 96 & 1647 \\
\hline & & & $\begin{array}{l}\text { \% within COUNTRY } \\
\text { CODE }\end{array}$ & $10.5 \%$ & $10.1 \%$ & $39.7 \%$ & $19.3 \%$ & $14.5 \%$ & $5.8 \%$ & $100.0 \%$ \\
\hline & & & $\%$ within EDUCATION & $100.0 \%$ & $100.0 \%$ & $\begin{array}{l}100.0 \% \\
307 \%\end{array}$ & $100.0 \%$ & $100.0 \%$ & $100.0 \%$ & $100.0 \%$ \\
\hline & & & $\%$ of Jotal & $10.5 \%$ & $10.1 \%$ & $397 \%$ & $19.3 \%$ & $14.5 \%$ & $58 \%$ & $100.0 \%$ \\
\hline \multirow[t]{16}{*}{ FEMALE } & \multirow{16}{*}{$\begin{array}{l}\text { COUNTEY } \\
\text { CODE }\end{array}$} & USA & $\begin{array}{l}\text { Count } \\
\text { \% within COUNTRY }\end{array}$ & & 4 & 9 & 18 & 16 & 1 & 48 \\
\hline & & & COOE & & $8.3 \%$ & $18.8 \%$ & $37.5 \%$ & $33.3 \%$ & $21 \%$ & $100.0 \%$ \\
\hline & & & \% within EDUCATION & & $65 \%$ & $5.1 \%$ & $15.4 \%$ & $20.8 \%$ & $3.2 \%$ & $9.5 \%$ \\
\hline & & & \% of Total & & $8 \%$ & $18 \%$ & $3.6 \%$ & $3.2 \%$ & $2 \%$ & $9.5 \%$ \\
\hline & & CANIADA & Count & 2 & 7 & 5 & 2 & 3 & 1 & 20 \\
\hline & & & $\begin{array}{l}\text { CODIhI COUNIR } \\
\text { CODE }\end{array}$ & $10.0 \%$ & $35.0 \%$ & $25.0 \%$ & $10.0 \%$ & $15.0 \%$ & $5.0 \%$ & $100.0 \%$ \\
\hline & & & \% within EOUCATION & $4.9 \%$ & $11.3 \%$ & $2.8 \%$ & $1.7 \%$ & $3.9 \%$ & $3.2 \%$ & $4.0 \%$ \\
\hline & & & $\%$ of Total & $.4 \%$ & $1.4 \%$ & $1.0 \%$ & $.4 \%$ & $.6 \%$ & $.2 \%$ & $4.0 \%$ \\
\hline & & CHINA & Count & 18 & 3 & 23 & & & 14 & 58 \\
\hline & & & $\begin{array}{l}\text { \% within COUNTRY } \\
\text { CODE }\end{array}$ & $31.0 \%$ & $5.2 \%$ & $39.7 \%$ & & & $24.1 \%$ & $100.0 \%$ \\
\hline & & & \% within EOUCATION & $439 \%$ & $4.8 \%$ & $13.1 \%$ & & & $45.2 \%$ & $11.5 \%$ \\
\hline & & & $\%$ of Total & $3.6 \%$ & $.6 \%$ & $46 \%$ & & & $2.8 \%$ & $11.5 \%$ \\
\hline & & TAWAN & Count & & 3 & 11 & 2 & & & 16 \\
\hline & & & $\begin{array}{l}\text { \% within COUNTRY } \\
\text { COOE }\end{array}$ & & $18.8 \%$ & $68.8 \%$ & $12.5 \%$ & & & $100.0 \%$ \\
\hline & & & \% within EOUCATION & & $4.8 \%$ & $6.3 \%$ & $1.7 \%$ & & & $32 \%$ \\
\hline & & & $\%$ of Total & & $6 \%$ & $2.2 \%$ & $4 \%$ & & & $3.2 \%$ \\
\hline
\end{tabular}


APPENDIX: Educational Level for Male and Female hanagers by Country/Region (cont)

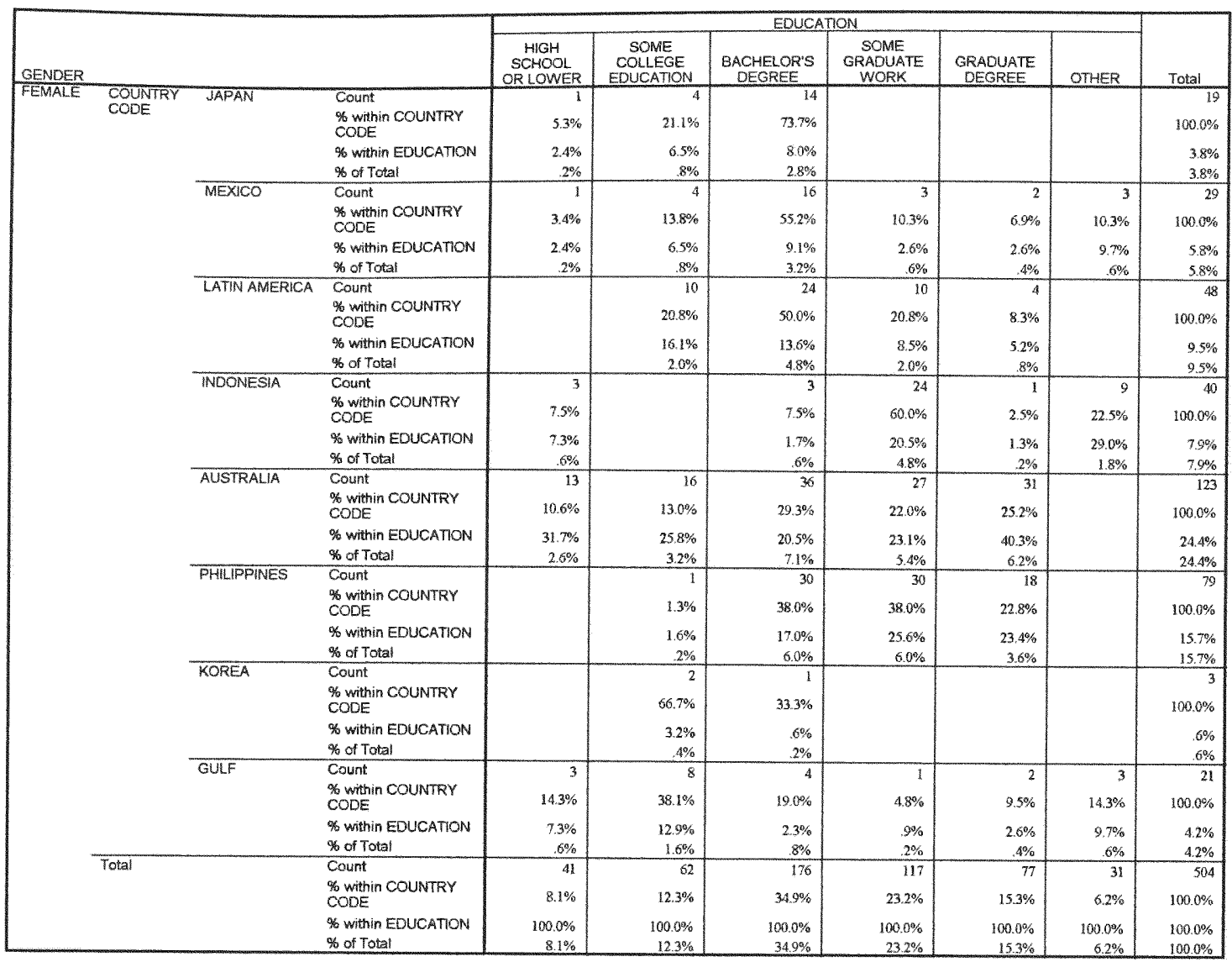

Chi-Square Tests: Educational Level for Male and Female Managers by Country/Region

\begin{tabular}{|ll|r|r|r|}
\hline GENDER & & Value & df & $\begin{array}{c}\text { Asymp. Sig. } \\
\text { (2-sided) }\end{array}$ \\
\hline MALE & Pearson Chi-Square & $992.332^{\mathrm{a}}$ & 55 & .000 \\
& Likelihood Ratio & 1040.586 & 55 & .000 \\
& Linear-by-Linear & 7.202 & 1 & .007 \\
& Association & 1647 & & .000 \\
& N of Valid Cases & $313.962^{\mathrm{b}}$ & 55 & .000 \\
\hline FEMALE & Pearson Chi-Square & 337.247 & 55 & .508 \\
& Likelihood Ratio & .438 & 1 & \\
& Linear-by-Linear & & & \\
& Association & & & \\
& N of Valid Cases & & & \\
\hline
\end{tabular}

a. 3 cells $(4.2 \%)$ have expected count less than 5 . The minimum expected count is 3.38 .

b. 40 cells $(55.6 \%)$ have expected count less than 5 . The minimum expected count is 18 . 
APPENDIX: Salary levels for Male and Female Managers by Country/Region

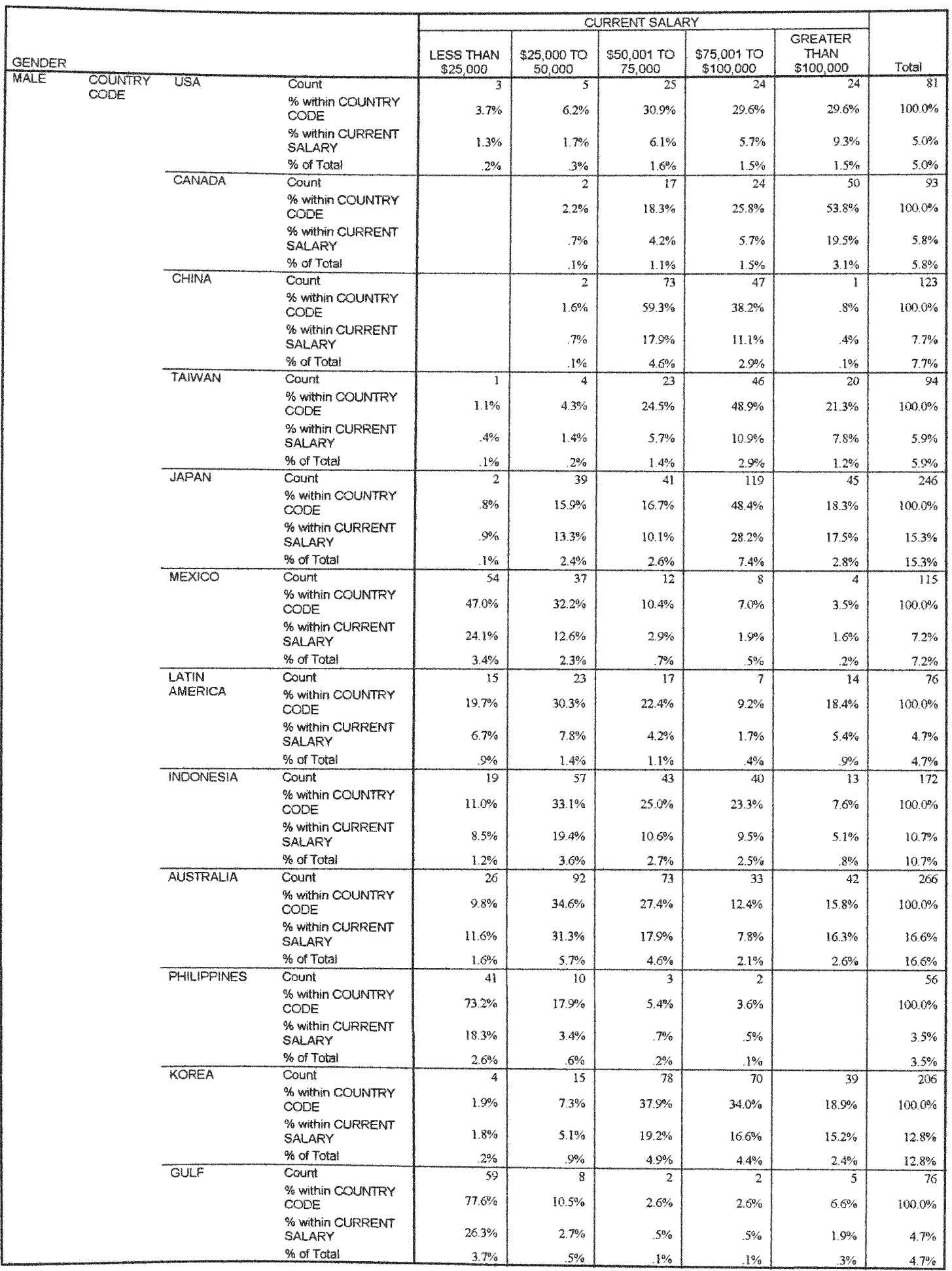


APPENDX: Salary levels for Male and Female Managers by CountryfRegion (cont.)

\begin{tabular}{|c|c|c|c|c|c|c|c|c|c|}
\hline \multirow[b]{2}{*}{ GENDER } & & & & \multicolumn{5}{|c|}{ CURRENT SALARY } & \multirow[b]{2}{*}{ Total } \\
\hline & & & & $\begin{array}{c}\text { LESS THAN } \\
\$ 25,000\end{array}$ & $\begin{array}{c}\$ 25,000 T 0 \\
50,000 \\
\end{array}$ & $\begin{array}{c}350,001 \mathrm{TO} \\
75,000 \\
\end{array}$ & $\begin{array}{c}\$ 75,001 T 0 \\
\$ 100,000 \\
\end{array}$ & $\begin{array}{c}\text { GREATER } \\
\text { THAN } \\
\$ 100,000 \\
\end{array}$ & \\
\hline \multirow[t]{4}{*}{ MALE } & \multirow{4}{*}{\multicolumn{2}{|c|}{ Total }} & Count & 224 & 294 & 407 & 422 & 257 & 1604 \\
\hline & & & $\begin{array}{l}\% \text { within COUNTRY } \\
\text { CODE }\end{array}$ & $14.0 \%$ & $18.3 \%$ & $25.4 \%$ & $263 \%$ & $16.0 \%$ & $100,0 \%$ \\
\hline & & & $\begin{array}{l}\% \text { within CURRENT } \\
\text { SALARY }\end{array}$ & $100.0 \%$ & $1000 \%$ & $100.0 \%$ & $1000 \%$ & $1000 \%$ & $100.0 \%$ \\
\hline & & & \% of Total & $14,0 \%$ & $18.3 \%$ & $254 \%$ & $263 \%$ & $16.0 \%$ & $1000 \%$ \\
\hline \multirow[t]{41}{*}{ FEMALE } & \multirow{41}{*}{$\begin{array}{l}\text { COUNTRY } \\
\text { COOE }\end{array}$} & \multirow[t]{4}{*}{ USA } & Count & 2 & 6 & 23 & 9 & 5 & 45 \\
\hline & & & $\begin{array}{l}\text { \% within COUNTRY } \\
\text { COOE }\end{array}$ & $4.4 \%$ & $13.3 \%$ & $51.1 \%$ & $20.0 \%$ & $11.1 \%$ & $1000 \%$ \\
\hline & & & $\begin{array}{l}\% \text { within CURRENT } \\
\text { SALARY }\end{array}$ & $1.1 \%$ & $49 \%$ & $19.3 \%$ & $14.3 \%$ & $21.7 \%$ & $8.9 \%$ \\
\hline & & & $\%$ of Total & $4 \%$ & $1.2 \%$ & $4.5 \%$ & $1.8 \%$ & $1.0 \%$ & $8.9 \%$ \\
\hline & & \multirow[t]{4}{*}{ CANADA } & Count & & 7 & 6 & 3 & 5 & $2 l$ \\
\hline & & & $\begin{array}{l}\text { \% within COUNTRY } \\
\text { COOE }\end{array}$ & & $33.3 \%$ & $28.6 \%$ & $14.3 \%$ & $23.8 \%$ & $1000 \%$ \\
\hline & & & $\begin{array}{l}\text { \% within CURRENT } \\
\text { SALARY }\end{array}$ & & $5.7 \%$ & $5.0 \%$ & $4.8 \%$ & $21.7 \%$ & $4.2 \%$ \\
\hline & & & \% of Total & & $1.4 \%$ & $1.2 \%$ & $.6 \%$ & $10 \%$ & $4.2 \%$ \\
\hline & & \multirow[t]{4}{*}{ CHINA } & Count & & & 38 & 23 & & 61 \\
\hline & & & $\begin{array}{l}\text { \% within COUNIRY } \\
\text { COOE }\end{array}$ & & & $62.3 \%$ & $37.7 \%$ & & $100.0 \%$ \\
\hline & & & $\begin{array}{l}\text { \% within CURRENT } \\
\text { SALARY }\end{array}$ & & & $31: 5 \%$ & $36.5 \%$ & & $12.1 \%$ \\
\hline & & & $\%$ of Total & & & $7.5 \%$ & $45 \%$ & & $12.1 \%$ \\
\hline & & \multirow[t]{4}{*}{ TAWAN } & Count & 5 & 1 & 2 & 6 & 1 & 15 \\
\hline & & & $\begin{array}{l}\% \text { within COUNTRY } \\
\text { CODE }\end{array}$ & $33.3 \%$ & $6.7 \%$ & $13.3 \%$ & $40.0 \%$ & $6.7 \%$ & $100.0 \%$ \\
\hline & & & $\begin{array}{l}\% \text { within CURRENT } \\
\text { SALARY }\end{array}$ & $2.8 \%$ & $8 \%$ & $1.7 \%$ & $9.5 \%$ & $4.3 \%$ & $3.0 \%$ \\
\hline & & & $\%$ of Total & $10 \%$ & $2 \%$ & $.4 \%$ & $1.2 \%$ & $.2 \%$ & $3.0 \%$ \\
\hline & & \multirow[t]{4}{*}{ JAPAN } & Count & & 10 & 6 & 2 & 1 & 19 \\
\hline & & & $\begin{array}{l}\text { \% within COUNIRY } \\
\text { CODE }\end{array}$ & & $52.6 \%$ & $31.6 \%$ & $10.5 \%$ & $5.3 \%$ & $100.0 \%$ \\
\hline & & & $\begin{array}{l}\text { \% within CURRENT } \\
\text { SALARY }\end{array}$ & & $8.2 \%$ & $5.0 \%$ & $3.2 \%$ & $4.3 \%$ & $3.8 \%$ \\
\hline & & & $\%$ of Total & & $2.0 \%$ & $1.2 \%$ & $.4 \%$ & $.2 \%$ & $3.8 \%$ \\
\hline & & \multirow[t]{3}{*}{ MEXICO } & $\begin{array}{l}\text { Count } \\
\text { \% within COUNTRY } \\
\text { CODE }\end{array}$ & $\begin{array}{r}27 \\
93.1 \%\end{array}$ & & $\begin{array}{r}1 \\
3.4 \%\end{array}$ & $\begin{array}{r}1 \\
3.4 \%\end{array}$ & & $\begin{array}{r}29 \\
100.0 \%\end{array}$ \\
\hline & & & $\begin{array}{l}\text { \% within CURRENT } \\
\text { SALARY }\end{array}$ & $15.1 \%$ & & $8 \%$ & $1.6 \%$ & & $5.7 \%$ \\
\hline & & & $\%$ of Total & $5.3 \%$ & & $.2 \%$ & $2 \%$ & & $5.7 \%$ \\
\hline & & \multirow[t]{3}{*}{$\begin{array}{l}\text { LATIN } \\
\text { AMERICA }\end{array}$} & $\begin{array}{l}\text { Gount } \\
\text { \% within COUNTRY } \\
\text { COOE }\end{array}$ & $\begin{array}{r}19 \\
42.2 \%\end{array}$ & $\begin{array}{r}14 \\
31.1 \%\end{array}$ & $\begin{array}{r}7 \\
15.6 \%\end{array}$ & & $11.1 \%$ & $\begin{array}{r}45 \\
1000 \%\end{array}$ \\
\hline & & & $\begin{array}{l}\% \text { within CURRENT } \\
\text { SALARY }\end{array}$ & $10.6 \%$ & $11.5 \%$ & $59 \%$ & & $21.7 \%$ & $8.9 \%$ \\
\hline & & & $\%$ of Total & $3.8 \%$ & $2.8 \%$ & $1.4 \%$ & & $1.0 \%$ & $8.9 \%$ \\
\hline & & \multirow[t]{4}{*}{ INDONESIA } & Count & 4 & 14 & 6 & 10 & 3 & 37 \\
\hline & & & $\begin{array}{l}\text { \% within COUNTRY } \\
\text { CODE }\end{array}$ & $10.8 \%$ & $37.8 \%$ & $16.2 \%$ & $27.0 \%$ & $8.1 \%$ & $1000 \%$ \\
\hline & & & $\begin{array}{l}\text { \% wihin CURRENT } \\
\text { SALARY }\end{array}$ & $2.2 \%$ & $11.5 \%$ & $5.0 \%$ & $15.9 \%$ & $13.0 \%$ & $7.3 \%$ \\
\hline & & & $\%$ of Total & $.8 \%$ & $2.8 \%$ & $1.2 \%$ & $20 \%$ & $.6 \%$ & $7.3 \%$ \\
\hline & & \multirow[t]{3}{*}{ AUSTRALIA } & $\begin{array}{l}\text { Count } \\
\text { \% whin COUNTRY } \\
\text { CODE }\end{array}$ & $\begin{array}{r}46 \\
33.3 \%\end{array}$ & $\begin{array}{r}57 \\
41.3 \%\end{array}$ & $\begin{array}{r}25 \\
18.1 \%\end{array}$ & $\begin{array}{r}7 \\
5.1 \%\end{array}$ & $\begin{array}{r}3 \\
2.2 \%\end{array}$ & $\begin{array}{r}138 \\
100.0 \%\end{array}$ \\
\hline & & & $\begin{array}{l}\text { \% within CURRENT } \\
\text { SALARY }\end{array}$ & $25.7 \%$ & $46.7 \%$ & $21.0 \%$ & $11.1 \%$ & $13.0 \%$ & $27.3 \%$ \\
\hline & & & \% of Total & $9.1 \%$ & $11.3 \%$ & $45 \%$ & $1.4 \%$ & $6 \%$ & $27.3 \%$ \\
\hline & & \multirow[t]{4}{*}{ PHLLIPPINES } & Count & 61 & 8 & 2 & 2 & & 73 \\
\hline & & & $\begin{array}{l}\text { \% within COUNTRY } \\
\text { COOE }\end{array}$ & $83.6 \%$ & $11.0 \%$ & $2.7 \%$ & $2.7 \%$ & & $100.0 \%$ \\
\hline & & & $\begin{array}{l}\text { \% within CURFENT } \\
\text { SALARY }\end{array}$ & $34.1 \%$ & $6.6 \%$ & $1.7 \%$ & $3.2 \%$ & & $14.4 \%$ \\
\hline & & & $\%$ of Total & $12.1 \%$ & $1.6 \%$ & $.4 \%$ & $4 \%$ & & $14.4 \%$ \\
\hline & & \multirow[t]{4}{*}{ KOREA } & Count & & 1 & 2 & & & 3 \\
\hline & & & $\begin{array}{l}\text { \% within COUNTRY } \\
\text { CODE }\end{array}$ & & $33.3 \%$ & $66.7 \%$ & & & $100.0 \%$ \\
\hline & & & $\begin{array}{l}\text { \% within CURRENT } \\
\text { SALARY }\end{array}$ & & $.8 \%$ & $1.7 \%$ & & & $6 \%$ \\
\hline & & & $\%$ of Total & & $2 \%$ & $4 \%$ & & & $.6 \%$ \\
\hline
\end{tabular}


APPENOIX: Salary levels for Male and Fernale Managers by Country/Region (cont)

\begin{tabular}{|c|c|c|c|c|c|c|c|c|}
\hline \multirow[b]{2}{*}{ GENOER } & & & \multicolumn{5}{|c|}{ CURRENT SALARY } & \multirow[b]{2}{*}{ Total } \\
\hline & & & $\begin{array}{l}\text { LESS THAN } \\
\$ 25,000\end{array}$ & $\begin{array}{c}\$ 25,000 \text { TO } \\
50,000\end{array}$ & $\begin{array}{c}\$ 50,00170 \\
75,000\end{array}$ & $\begin{array}{c}\$ 75,001 \mathrm{TO} \\
\$ 100,000\end{array}$ & $\begin{array}{l}\text { GREATER } \\
\text { THAN } \\
\$ 100,000\end{array}$ & \\
\hline \multirow[t]{8}{*}{ FEMALE } & COUNIRY GULF & count & 15 & 4 & 1 & & & 20 \\
\hline & & $\begin{array}{l}\text { \% within COUNTRY } \\
\text { CODE }\end{array}$ & $75.0 \%$ & $20.0 \%$ & $5.0 \%$ & & & $100.0 \%$ \\
\hline & & $\begin{array}{l}\text { \% within CURRENT } \\
\text { SALARY }\end{array}$ & $8.4 \%$ & $3.3 \%$ & $8 \%$ & & & $4.0 \%$ \\
\hline & & $\%$ of Total & $3.0 \%$ & $.8 \%$ & $.2 \%$ & & & $40 \%$ \\
\hline & Total & Count & 179 & 122 & 119 & 63 & 23 & 506 \\
\hline & & $\begin{array}{l}\text { \% within COUNTRY } \\
\text { CODE }\end{array}$ & $35.4 \%$ & $24.1 \%$ & $23.5 \%$ & $12.5 \%$ & $45 \%$ & $100.0 \%$ \\
\hline & & $\begin{array}{l}\% \text { within CURRENT } \\
\text { SALARY }\end{array}$ & $100.0 \%$ & $100.0 \%$ & $100.0 \%$ & $100.0 \%$ & $1000 \%$ & $100.0 \%$ \\
\hline & & $\%$ of Total & $35.4 \%$ & $24.1 \%$ & $23.5 \%$ & $125 \%$ & $45 \%$ & $100.0 \%$ \\
\hline
\end{tabular}

Chi-Square Tests: Salary Levels for Male and Female Managers by Country/Region

\begin{tabular}{|ll|r|r|r|}
\hline GENDER & & \multicolumn{1}{|c|}{ Value } & df & $\begin{array}{c}\text { Asymp. Sig. } \\
\text { (2-sided) }\end{array}$ \\
\hline MALE & Pearson Chi-Square & $1106.419^{\circ}$ & 44 & .000 \\
& Likelihood Ratio & 994.861 & 44 & .000 \\
& Linear-by-Linear & 151.768 & 1 & .000 \\
& Association & 1604 & & .000 \\
& N of Valid Cases & $389.529^{\mathrm{b}}$ & 44 & .000 \\
\hline FEMALE & Pearson Chi-Square & 421.859 & 44 & .000 \\
& Likelihood Ratio & 135.725 & 1 & \\
& Linear-by-Linear & 506 & & \\
& Association & & \\
& N of Valid Cases & & & \\
\end{tabular}

a. 0 cells $(.0 \%)$ have expected count less than 5 . The minimum expected count is 7.82 .

b. 28 cells $(46.7 \%)$ have expected count less than 5 . The minimum expected count is 14 . 
APPENDIX: Age Categories for Male and Female Managers by Country/Region

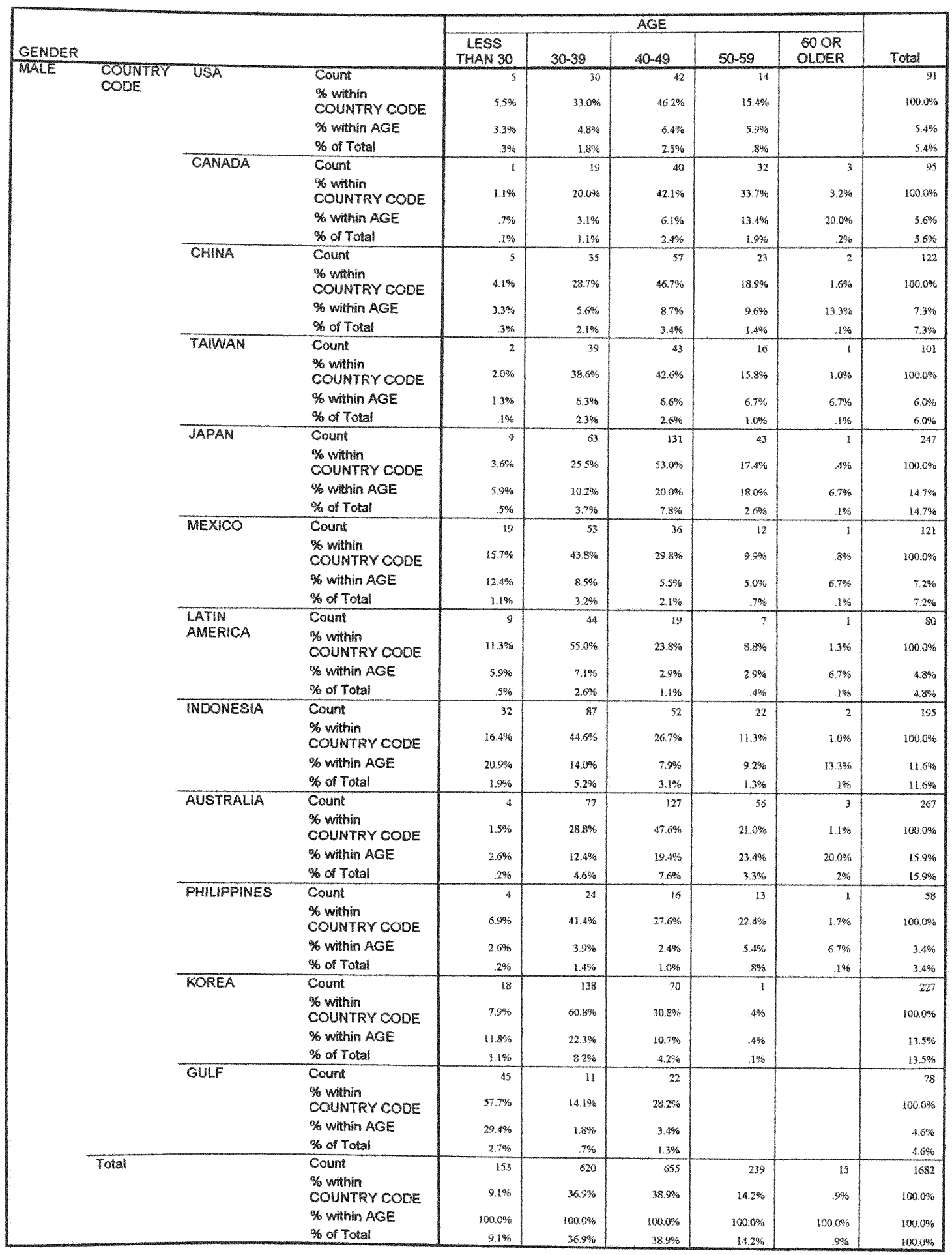


APPENDIX: Age Categories for Male and Female Managers by Country/Region (cont.)

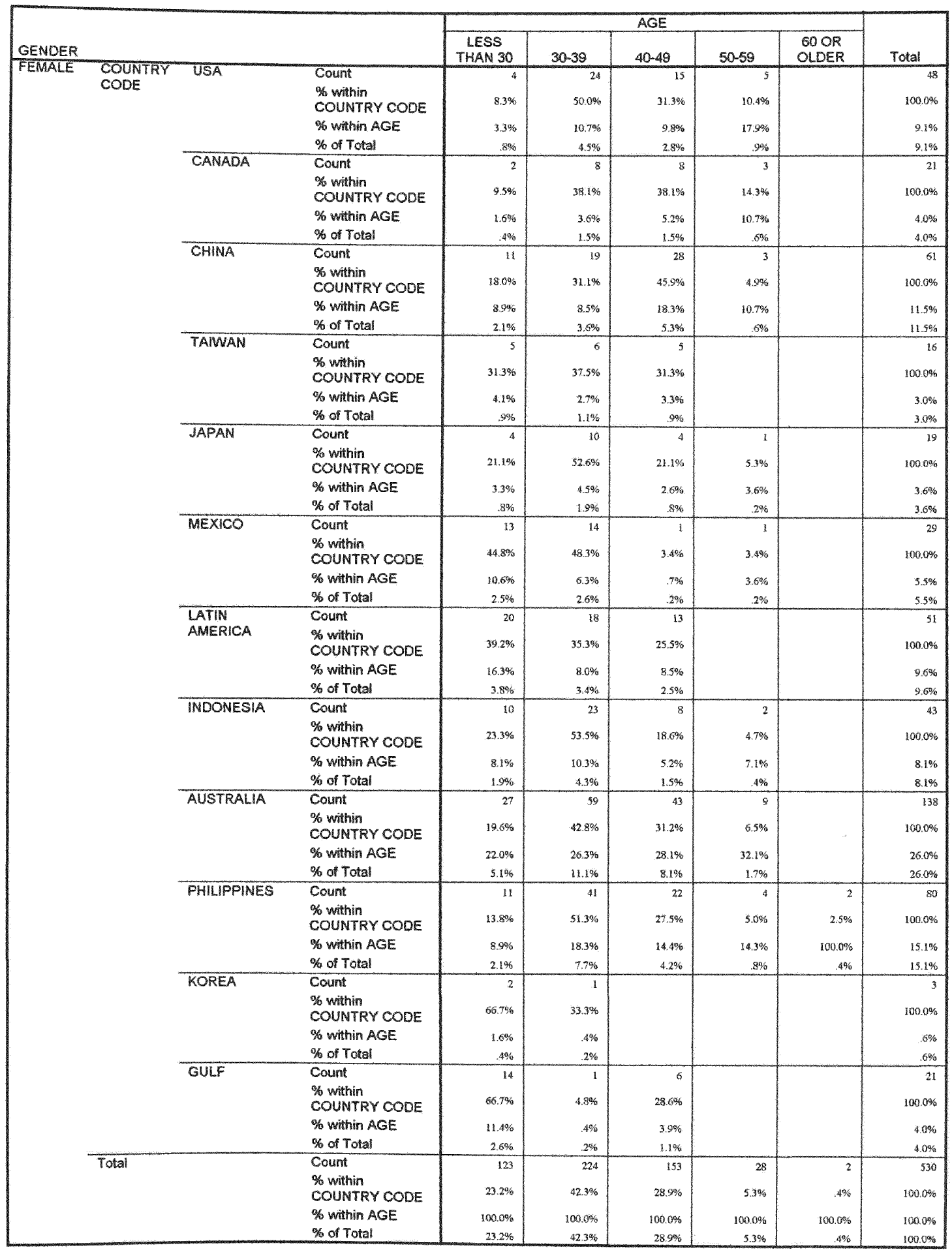


Chi-Square Tests: Age Categories for Male and Female Managers by Country/Region

\begin{tabular}{|ll|r|r|r|}
\hline GENDER & Value & df & \multicolumn{1}{|c|}{$\begin{array}{c}\text { Asymp. Sig. } \\
\text { (2-sided) }\end{array}$} \\
\hline MALE & Pearson Chi-Square & $486.607^{\circ}$ & 44 & .000 \\
& Likelihood Ratio & 420.344 & 44 & .000 \\
& Linear-by-Linear & 95.837 & 1 & .000 \\
& Association & 1682 & & .000 \\
& N of Valid Cases & $95.179^{\circ}$ & 44 & .000 \\
\hline FEMALE & Pearson Chi-Square & 96.942 & 44 & .021 \\
& Likelihood Ratio & 5.307 & 1 & \\
& Linear-by-Linear & 530 & & \\
& Association & & & \\
& N of Valid Cases & & & \\
\hline
\end{tabular}

a. 12 cells $(20.0 \%)$ have expected count less than 5 . The minimum expected count is .52 .

b. 31 cells $(51.7 \%)$ have expected count less than 5 . The minimum expected count is 01 . 
APPENDIX: Multivariate Tests

Item Bias Detection: Score Level by Country

\begin{tabular}{|ll|l|r|}
\hline & & Value Label & N \\
\hline LEVELS & 1 & $9-15$ & 24 \\
& 2 & $15-20$ & 130 \\
& 3 & $21-25$ & 304 \\
& 4 & $26-30$ & 689 \\
& 5 & $31-35$ & 652 \\
& 6 & $36-40$ & 327 \\
& 7 & $41-44$ & 97 \\
COUNTRY & 1 & USA & 141 \\
CODE & 2 & CANADA & 117 \\
& 3 & CHINA & 181 \\
4 & TAINAN & 118 \\
& 5 & JAPAN & 269 \\
6 & MEXICO & 172 \\
7 & LATNN & 133 \\
& 8 & AMERICA & 185 \\
10 & ANDONESIA & 422 \\
& AUSTRALIA & 159 \\
11 & PHILIPINNES & 232 \\
& 14 & KOREA & 94 \\
\hline
\end{tabular}

APPENDIX: Multivariate Tests: Item Bias Detection. Score Level Analysis by Country/Regionc

\begin{tabular}{|ll|r|r|r|r|r|}
\hline Effect & & Value & \multicolumn{1}{c|}{$F$} & Hypothesis df & Error df & Sig. \\
\hline Intercept & Pillai's Trace & .988 & $19403.284^{\mathrm{a}}$ & 9.000 & 2132.000 & .000 \\
& Wilks' Lambda & .012 & $19403.284^{\mathrm{a}}$ & 9.000 & 2132.000 & .000 \\
& Hotelling's Trace & 81.909 & $19403.284^{\mathrm{a}}$ & 9.000 & 2132.000 & .000 \\
& Roy's Largest Root & 81.909 & $19403.284^{\mathrm{a}}$ & 9.000 & 2132.000 & .000 \\
\hline LEVELS & Pillai's Trace & .942 & 44.242 & 54.000 & 12822.000 & .000 \\
& Wilks' Lambda & .083 & 126.718 & 54.000 & 10875.704 & .000 \\
& Hotelling's Trace & 10.743 & 423.812 & 54.000 & 12782.000 & .000 \\
& Roy's Largest Root & 10.715 & $2544.180^{\mathrm{b}}$ & 9.000 & 2137.000 & .000 \\
\hline CoCODE & Pillai's Trace & .228 & 5.062 & 99.000 & 19260.000 & .000 \\
& Wilks' Lambda & .789 & 5.202 & 99.000 & 15046.346 & .000 \\
& Hotelling's Trace & .247 & 5.316 & 99.000 & 19172.000 & .000 \\
& Roy's Largest Root & .126 & $24.500^{\mathrm{b}}$ & 11.000 & 2140.000 & .000 \\
& Pillai's Trace & .449 & 1.730 & 585.000 & 19260.000 & .000 \\
& Wiks' Lambda & .624 & 1.766 & 585.000 & 19042.767 & .000 \\
& Hotelling's Trace & .496 & 1.805 & 585.000 & 19172.000 & .000 \\
& Roy's Largest Root & .194 & $6.379^{\mathrm{b}}$ & 65.000 & 2140.000 & .000 \\
\hline
\end{tabular}

a. Exact statistic

b. The statistic is an upper bound on $F$ that yields a lower bound on the significance level.

c. Design: Intercept+LEVELS+COCOOE+LEVELS * COCODE 
APPENDIX: Multivariate tests: Item Bias Detection. Score Level Analysis by Country/Region

\begin{tabular}{|c|c|c|c|c|c|c|}
\hline Source & Dependent Variable & $\begin{array}{c}\text { Type III Sum } \\
\text { of Squares }\end{array}$ & $d f$ & Mean Square & $F$ & Sig. \\
\hline \multirow[t]{9}{*}{ Corrected Model } & SPECIFIC GOALS & $1127.534^{8}$ & 82 & 13.750 & 23.089 & .000 \\
\hline & STANDARDS & $1102827^{\circ}$ & 82 & 13.49 & 24.300 & .000 \\
\hline & WORK GOALS & $1281.874^{\mathrm{C}}$ & 82 & 15.633 & 26.371 & .000 \\
\hline & FRIENDLY & $1277.939^{d}$ & 82 & 15.585 & 25.193 & .000 \\
\hline & $\begin{array}{l}\text { RECOGNIZE/REWARD } \\
\text { PERFORMANCE }\end{array}$ & $1394.224^{8}$ & 82 & 17.003 & 30.012 & .000 \\
\hline & LISTEN & $1645.538^{f}$ & 82 & 20.068 & 37.664 & .000 \\
\hline & RESPECT & $2271.836^{\circ}$ & 82 & 27.705 & 48.106 & .000 \\
\hline & NOTRUST & $1043.519^{\mathrm{h}}$ & 82 & 12.726 & 15.415 & .000 \\
\hline & $\begin{array}{l}\text { SUGGESTIONS TO } \\
\text { TOP MGT }\end{array}$ & $1019.088^{i}$ & 82 & 12.428 & 19.730 & 000 \\
\hline \multirow[t]{9}{*}{ Intercept } & SPECIFIC GOALS & 3566.583 & 1 & 3566.583 & 5988.781 & .000 \\
\hline & STANDARDS & 4452.057 & 1 & 4452.057 & 8043.879 & .000 \\
\hline & WORK GOALS & 4330.974 & 1 & 4330.974 & 7306,128 & .000 \\
\hline & FRIENDLY & 4874.723 & 1 & 4874.723 & 7880,114 & .000 \\
\hline & $\begin{array}{l}\text { RECOGNIZE/REWARD } \\
\text { PERFORMANCE }\end{array}$ & 3758.420 & 1 & 3758.420 & 6534.143 & .000 \\
\hline & LISTEN & 4223.914 & 1 & 4223.914 & 7927.669 & .000 \\
\hline & RESPECT & 4711.393 & 1 & 4711393 & 8180.573 & .000 \\
\hline & NOTRUST & 3109.876 & 1 & 3109.876 & 3767.140 & .000 \\
\hline & $\begin{array}{l}\text { SUGGESTIONS TO } \\
\text { TOPMGT }\end{array}$ & 4147.977 & 1 & 4147.977 & 6585.155 & .000 \\
\hline \multirow[t]{9}{*}{ LEVELS } & SPECIFIC GOALS & 623.717 & 6 & 103.953 & 174.551 & .000 \\
\hline & STANDARDS & 565.273 & 6 & 94.212 & 170.221 & .000 \\
\hline & WORK GOALS & 675,404 & 6 & 112.567 & 189.895 & .000 \\
\hline & FRIENDLY & 543.214 & 6 & 90.536 & 146.353 & .000 \\
\hline & $\begin{array}{l}\text { RECOGNIZEIREWARD } \\
\text { PERFORMANCE }\end{array}$ & 776.537 & 6 & 129.423 & 228.450 & .000 \\
\hline & LISTEN & 787.878 & 6 & 131.313 & 246.455 & .000 \\
\hline & RESPECT & 462.499 & 6 & 77.083 & 133.842 & .000 \\
\hline & NO TRUST & 180.856 & 6 & 30.143 & 36.513 & .000 \\
\hline & $\begin{array}{l}\text { SUGGESTIONS TO } \\
\text { TOPMGT }\end{array}$ & 528.556 & 6 & 88.093 & 139.852 & .000 \\
\hline \multirow[t]{9}{*}{ COCODE } & SPECIFIC GOALS & 15.963 & i1 & 1.451 & 2.437 & .005 \\
\hline & STANDARDS & 18.157 & 11 & 1.651 & 2.982 & .001 \\
\hline & WORK GOALS & 21.956 & $n$ & 1.996 & 3.367 & 600 \\
\hline & FRIENDLY & 26.882 & 11 & 2.444 & 3950 & .000 \\
\hline & $\begin{array}{l}\text { RECOGNIZE/REWARD } \\
\text { PERFORMANCE }\end{array}$ & 6931 & 11 & .630 & 1.112 & .347 \\
\hline & LISTEN & 29.460 & 11 & 2.678 & 5.027 & .000 \\
\hline & RESPECT & 133.160 & 11 & 12.105 & 21.019 & .000 \\
\hline & NOTRUST & 105.469 & 11 & 9.588 & 11.614 & .000 \\
\hline & $\begin{array}{l}\text { SUGGESTIONS TO } \\
\text { TOP MGT }\end{array}$ & 12614 & 11 & 1.147 & 1.820 & .046 \\
\hline \multirow[t]{9}{*}{ LEVELS * COCOOE } & SPECIFIC GOALS & 43.498 & 65 & 669 & 1124 & .235 \\
\hline & STANDARDS & 51.842 & 65 & .798 & 1.441 & .013 \\
\hline & WORK GOALS & 42.940 & 65 & .661 & 1.114 & .250 \\
\hline & FRIENDLY & 36.544 & 65 & .562 & .909 & .682 \\
\hline & $\begin{array}{l}\text { RECOGNIZE/REWARD } \\
\text { PERFORMANCE }\end{array}$ & 34.955 & 65 & .538 & .949 & .592 \\
\hline & LISTEN & 43.587 & 65 & .671 & 1.259 & .082 \\
\hline & RESPECT & 223.743 & 65 & 3.442 & 5.977 & .000 \\
\hline & NO TRUST & 146.303 & 65 & 2.259 & 2736 & .000 \\
\hline & $\begin{array}{l}\text { SUGGESTIONS TO } \\
\text { TOPMGT } \\
\end{array}$ & 44.878 & 65 & .690 & 1096 & .282 \\
\hline
\end{tabular}


APPENDIX: Multivariate tests: Item Bias Detection. Score Level Analysis by Country/Region (cont)

\begin{tabular}{|c|c|c|c|c|c|c|}
\hline Source & Dependent Variable & $\begin{array}{l}\text { Type III Sum } \\
\text { of Squares }\end{array}$ & $d f$ & Mean Square & $F$ & Sig. \\
\hline Error & $\begin{array}{l}\text { SPECIFIC GOALS } \\
\text { STANDARDS } \\
\text { WORK GOALS } \\
\text { FRIENDLY } \\
\text { RECOGNIZE/REWARO } \\
\text { PERFORMANCE } \\
\text { LISTEN } \\
\text { RESPECT } \\
\text { NOTRUST } \\
\text { SUGGESTIONSTO } \\
\text { TOPMGT }\end{array}$ & $\begin{array}{l}1274.464 \\
1184.429 \\
1268.563 \\
1323.827 \\
1212.367 \\
1140.206 \\
1232.478 \\
1766.628 \\
1347.982\end{array}$ & $\begin{array}{l}2140 \\
2140 \\
2140 \\
2140 \\
2140 \\
2140 \\
2140 \\
2140 \\
2140\end{array}$ & $\begin{array}{r}.596 \\
.553 \\
.593 \\
.619 \\
.567 \\
.533 \\
.576 \\
.826 \\
.630\end{array}$ & & \\
\hline Total & $\begin{array}{l}\text { SPECIFIC GOALS } \\
\text { STANDARDS } \\
\text { WORK GOALS } \\
\text { FRIENDLY } \\
\text { RECOGNIZEREWARD } \\
\text { PERFORMANCE } \\
\text { LISTEN } \\
\text { RESPECT } \\
\text { NOTRUST } \\
\text { SUGGESTIONSTO } \\
\text { TOPMGT } \\
\end{array}$ & $\begin{array}{l}23906.000 \\
307900000 \\
285060000 \\
32014000 \\
25907.000 \\
30042.000 \\
28653.000 \\
21939.000 \\
267150000\end{array}$ & $\begin{array}{l}2223 \\
2223 \\
2223 \\
2223 \\
2223 \\
2223 \\
2223 \\
2223 \\
2223\end{array}$ & & & \\
\hline Corrected Total & $\begin{array}{l}\text { SPECIFIC GOALS } \\
\text { STANDARDS } \\
\text { WORK GOALS } \\
\text { FRIENDLY } \\
\text { RECOGNIZE/REWARD } \\
\text { PERFORMANCE } \\
\text { LISTEN } \\
\text { RESPECT } \\
\text { NO TRUST } \\
\text { SUGGESTIONS TO } \\
\text { TOPMGT }\end{array}$ & $\begin{array}{l}2401.998 \\
2287.256 \\
2550.437 \\
2601.766 \\
2606.591 \\
2785.744 \\
3504.314 \\
2810.147 \\
2367.070\end{array}$ & $\begin{array}{l}2222 \\
2222 \\
2222 \\
2222 \\
2222 \\
2222 \\
2222 \\
2222 \\
2222\end{array}$ & & & \\
\hline
\end{tabular}

a. $R$ Squared $=.469($ Adjusted $R$ Squared $=.449)$

b. $R$ Squared $=.482$ (Adjusted $R$ Squared $=.462$ )

c. $R$ Squared $=.503$ (Adjusted $R$ Squared $=.484$ )

d. $R$ Squared $=.491$ (Adjusted $R$ Squared $=.472)$

e. $R$ Squared $=.535$ (Adjusted $R$ Squared $=.517$ )

f. $R$ Squared $=.591$ (Adjusted $R$ Squared $=.575$ )

g. $R$ Squared $=.648$ (Adjusted $R$ Squared $=.635$ )

h. $R$ Squared $=.371$ (Adjusted $R$ Squared $=.347$ )

i. $R$ Squared $=.431$ (Adjusted R Squared $=.409$ ) 
APPENDIX: Between-Subjects Factors: Gender Effects on Leader Behavior Items by Country/Region

\begin{tabular}{|ll|l|r|}
\hline & & Value Label & N \\
\hline COUNTRY & 1 & USA & 139 \\
CODE & 2 & CANADA & 115 \\
& 3 & CHINA & 174 \\
& 4 & TAIWAN & 117 \\
5 & JAPAN & 262 \\
6 & MEXICO & 145 \\
7 & LATIN & 121 \\
& AMERICA & 186 \\
& 10 & INDONESIA & 397 \\
11 & AUSTRALIA & 135 \\
12 & PHILIPPINES & 227 \\
14 & KOREA & 93 \\
\hline
\end{tabular}

APPENDIX: Multivariate Tests: Gender Effects on Leader Behavior Items by Country/Regionc

\begin{tabular}{|c|c|c|c|c|c|c|c|}
\hline Effect & & Value & $F$ & Hypothesis of & Error df & Sig. & Eta Squared \\
\hline \multirow[t]{4}{*}{ Intercept } & Pillai's Trace & .754 & $710.780^{8}$ & 9.000 & 2090.000 & .000 & .754 \\
\hline & Wilks' Lambda & .246 & $710.780^{\mathrm{a}}$ & 9.000 & 2090.000 & .000 & .754 \\
\hline & Hotelling's Trace & 3.061 & $710.780^{\mathrm{a}}$ & 9.000 & 2090.000 & .000 & .754 \\
\hline & Roy's Largest Root & 3.061 & $710.780^{\mathrm{a}}$ & 9.000 & 2090.000 & .000 & .754 \\
\hline \multirow[t]{4}{*}{ SEX } & Pillai's Trace & .004 & $1.027^{\mathrm{a}}$ & 9.000 & 2090.000 & .416 & .004 \\
\hline & Wiks' Lambda & .996 & $1.027^{\mathrm{a}}$ & 9.000 & 2090.000 & .416 & .004 \\
\hline & Hoteling's Trace & .004 & $1.027^{\mathrm{a}}$ & 9.000 & 2090,000 & .416 & .004 \\
\hline & Roy's Largest Root & .004 & $1.027^{8}$ & 9,000 & 2090.000 & .416 & .004 \\
\hline \multirow[t]{4}{*}{ COCODE } & Pillai's Trace & 1.029 & 24.613 & 99.000 & 18882.000 & .000 & .114 \\
\hline & Wilks Lambda & .276 & 29.883 & 99.000 & 14750.161 & .000 & .133 \\
\hline & Hotelling's Trace & 1.689 & 35.636 & 99.000 & 18794.000 & .000 & .158 \\
\hline & Roy's Largest Root & 1.069 & $203.965^{b}$ & 11.000 & 2098.000 & .000 & .517 \\
\hline
\end{tabular}
a. Exact statistic
b. The statistic is an upper bound on $F$ that yields a lower bound on the significance level.
c. Design: Intercept+SEX+COCODE 
APPENDIX: Tests of Beween-Subjects Effects: Gender Effects on Leader Behavior ftems by Country/region

\begin{tabular}{|c|c|c|c|c|c|c|c|}
\hline Source & Dependent Variable & $\begin{array}{l}\text { Type Ill Sum } \\
\text { of Squares }\end{array}$ & df & Mean Square & $F$ & Sig. & Eta Squared \\
\hline \multirow[t]{9}{*}{ Corrected Model } & SPECIFIC GOALS & $121.000^{9}$ & 12 & 10.083 & 9.686 & .000 & .052 \\
\hline & STANDARDS & $151,965^{\mathrm{b}}$ & 12 & 12.664 & 13.092 & .000 & .070 \\
\hline & WORK GOALS & $127.151^{a}$ & 12 & 10.596 & 9.620 & .000 & .052 \\
\hline & FRIENDLY & $296.113^{c}$ & 12 & 24.676 & 23.834 & .000 & .120 \\
\hline & $\begin{array}{l}\text { RECOGNIZE/REWARD } \\
\text { PERFORMANCE }\end{array}$ & $64.667^{d}$ & 12 & 5.389 & 4.678 & .000 & .026 \\
\hline & LISTEN & $406.406^{\mathrm{e}}$ & 12 & 33.867 & 31.930 & .000 & .154 \\
\hline & RESPECT & $1411.208^{f}$ & 12 & 117.601 & 129.707 & .000 & .426 \\
\hline & NOTRUST & 555.8419 & 12 & 46.320 & 45.094 &, 000 & .205 \\
\hline & $\begin{array}{l}\text { SUGGESTIONS TO TOP } \\
\text { MGT }\end{array}$ & $116.556^{h}$ & 12 & 9.713 & 9.471 & .000 & .051 \\
\hline \multirow[t]{9}{*}{ Intercept } & SPECIFIC GOALS & 1980.034 & 1 & 1980.034 & 1901.930 & .000 & .475 \\
\hline & STANDARDS & 2418.489 & 1 & 2418.489 & 2500.290 & .000 & .544 \\
\hline & WORK GOALS & 2384.189 & 1 & 2384.189 & 2164.604 & .000 & .508 \\
\hline & FRIENDLY & 2710.895 & 1 & 2710.895 & 2618.415 & .000 & .555 \\
\hline & $\begin{array}{l}\text { RECOGNIZE/REWARD } \\
\text { PERFORMANCE }\end{array}$ & 2180.068 & 1 & 2180.068 & 1892.623 & .000 & .474 \\
\hline & LISTEN & 2449.289 & 1 & 2449.289 & 2309.183 & .000 & .524 \\
\hline & RESPECT & 2709.788 & 1 & 2709.788 & 2988.739 & .000 & .588 \\
\hline & NOTRUST & 1634.377 & 1 & 1634.377 & 1590.755 & .000 & .431 \\
\hline & $\begin{array}{l}\text { SUGGESTIONS TO TOP } \\
\text { MGT }\end{array}$ & 2334.188 & 1 & 2334.188 & 2276.120 & .000 & .520 \\
\hline \multirow[t]{9}{*}{ SEX } & SPECIFIC GOALS & .565 & 1 & .565 & .543 & .461 & .000 \\
\hline & STANDARDS & .420 & 1 & .420 & .434 & .510 & .000 \\
\hline & WORK GOALS & 1.059 & 1 & 1.059 & .962 & .327 & .000 \\
\hline & FRIENDLY & 1.145 & 1 & 1.145 & 1.106 & .293 & .001 \\
\hline & $\begin{array}{l}\text { RECOGNIZE/REWARD } \\
\text { PERFORMANCE }\end{array}$ & 1.203 & 1 & 1.203 & 1.045 & .307 & .000 \\
\hline & LISTEN & 1.280 & 1 & 1.280 & 1.207 & .272 & .001 \\
\hline & RESPECT & 3.193 & 1 & 3.193 & 3.521 & .061 & .002 \\
\hline & NOTRUST & .484 & 1 & .484 & .471 & .493 & .000 \\
\hline & $\begin{array}{l}\text { SUGGESTIONS TO TOP } \\
\text { MGT }\end{array}$ & 3.104 & 1 & 3.104 & 3.027 & .082 & .001 \\
\hline \multirow[t]{9}{*}{ COCODE } & SPECIFIC GOALS & 120.893 & 11 & 10.990 & 10.557 & .000 & .052 \\
\hline & STANDARDS & 145.739 & 11 & 13,249 & 13.697 & .000 & .067 \\
\hline & WORK GOALS & 124.823 & 11 & 11.348 & 10.302 & .000 & .051 \\
\hline & FRIENDLY & 284.386 & 11 & 25.853 & 24.971 & .000 & 116 \\
\hline & $\begin{array}{l}\text { RECOGNIZE/REWARD } \\
\text { PERFORMANCE }\end{array}$ & 64.625 & 11 & 5.875 & 5.100 & .000 & .026 \\
\hline & LISTEN & 393.163 & 11 & 35.742 & 33.698 & .000 & .150 \\
\hline & RESPECT & 1410.053 & 11 & 128.187 & 141.382 & .000 & .426 \\
\hline & NOTRUST & 555.740 & 11 & 50.522 & 49.173 & .000 & .205 \\
\hline & $\begin{array}{l}\text { SUGGESTIONS TO TOP } \\
\text { MGT }\end{array}$ & 145.743 & 11 & 10.522 & 10.260 & .000 & .051 \\
\hline \multirow[t]{9}{*}{ Error } & SPECIFIC GOALS & 2184.156 & 2098 & 1.041 & & & \\
\hline & STANDARDS & 2029.360 & 2098 & .967 & & & \\
\hline & WORK GOALS & 2310.828 & 2098 & 1.101 & & & \\
\hline & FRIENDLY & 2172.099 & 2098 & 1.035 & & & \\
\hline & $\begin{array}{l}\text { RECOGNIZE/REWARD } \\
\text { PERFORMANCE }\end{array}$ & 2416.638 & 2098 & 1.152 & & & \\
\hline & LISTEN & 2225.292 & 2098 & 1.061 & & & \\
\hline & RESPECT & 1902.185 & 2098 & 907 & & & \\
\hline & NOTRUST & 2155.534 & 2098 & 1.027 & & & \\
\hline & $\begin{array}{l}\text { SUGGESTIONS TO TOP } \\
\text { MGT }\end{array}$ & 2151.524 & 2098 & 1.026 & & & \\
\hline
\end{tabular}


APPENDIX: Tests of Between-Subjects Effects: Gender Effects on Leader Behavior Items by Country/region (cont.)

\begin{tabular}{|c|c|c|c|c|c|c|c|}
\hline Source & Dependent Variable & $\begin{array}{l}\text { Type lli Sum } \\
\text { of Squares }\end{array}$ & df & Mean Square & $\mathrm{F}$ & Sig. & Eta Squared \\
\hline \multirow[t]{9}{*}{ Total } & SPECIFIC GOALS & 22840.000 & 2111 & & & & \\
\hline & STANDARDS & 29277.000 & 2111 & & & & \\
\hline & WORK GOALS & 27118.000 & 2111 & & & & \\
\hline & FRIENDLY & 30336.000 & 2111 & & & & \\
\hline & $\begin{array}{l}\text { RECOGNIZE/REWARD } \\
\text { PERFORMANCF }\end{array}$ & 24670.000 & 2111 & & & & \\
\hline & $\begin{array}{l}\text { PERFORMANCE } \\
\text { LISTEN }\end{array}$ & 28565.000 & 2141 & & & & \\
\hline & RESPECT & 26985.000 & 2111 & & & & \\
\hline & NOTRUST & 21062.000 & 2111 & & & & \\
\hline & $\begin{array}{l}\text { SUGGESTIONS TO TOP } \\
\text { MGT }\end{array}$ & 25513.000 & 2111 & & & & \\
\hline \multirow[t]{9}{*}{ Corrected Total } & SPECIFIC GOALS & 2305.156 & 2110 & & & & \\
\hline & STANDARDS & 2181.325 & 2110 & & & & \\
\hline & WORK GOALS & 2437.979 & 2110 & & & & \\
\hline & FRIENDLY & 2468.212 & 2110 & & & & \\
\hline & $\begin{array}{l}\text { RECOGNIZE/REWARD } \\
\text { PERFORMANCE }\end{array}$ & 2481.305 & 2110 & & & & \\
\hline & LISTEN & 2631.698 & 2110 & & & & \\
\hline & RESPECT & 3313.394 & 2110 & & & & \\
\hline & NO TRUST & 2711.372 & 2110 & & & & \\
\hline & $\begin{array}{l}\text { SUGGESTIONS TO TOP } \\
\text { MGT }\end{array}$ & & 2110 & & & & \\
\hline
\end{tabular}
a. $R$ Squared $=.052$ (Adjusted $R$ Squared $=.047$ )
b. $R$ Squared $=.070$ (Adjusted $R$ Squared $=.064)$
c. $R$ Squared $=.120$ (Adjusted $R$ Squared $=.115$ )
d. $R$ Squared $=.026$ (Adjusted $R$ Squared $=.020$ )
e. $R$ Squared $=.154$ (Adjusted $R$ Squared $=.150$ )
f. $R$ Squared $=.426$ (Adjusted $R$ Squared $=.423$ )
g. $R$ Squared $=.205$ (Adjusted $R$ Squared $=.200$ )
h. $R$ Squared $=.051$ (Adjusted $R$ Squared $=.046$ ) 
FIT MEASURES

Discrepancy

Degrees of freedom

$P$

Number of parameters

Discrepancy / df

RMR

GFI

Adjusted GFI

Parsimony-adjusted GFI

Normed fit index

Relative fit index

Incremental fit index

Tucker-Lewis index

Comparative fit index

Parsimony ratio

Parsimony-adjusted NFI

Parsimony-adjusted CFI

Noncentrality parameter estimate

NCP lower bound

NCP upper bound

FMIN

FO

Fo lower bound

FO upper bound

RMSEA

RMSEA lower bound

RMSEA upper bound

$P$ for test of close fit

Akaike information criterion (AIC)

Browne-Cudeck criterion

Bayes information criterion

Consistent AIC

Expected cross validation index

ECVI lower bound

ECVI upper bound

MECVI

Hoelter .05 index

Hoelter .01 index
Model fit Saturated Independence 979.997

286

0

308

3.427

0

54828.563

495

0
99

110.765

594$$
110.765
$$

0.982

0.969

0.987

0.978

0.987

0.578

0.567

0.57

693.997

602.578

792.998

0.46

0.326

0.283

0.372

0.034

0.031

0.036 
APPENDIX: MODEL FIT INDICES AMOS 4.0

HYPOTHESIS 2: EQUALITY OF FACTOR LOADINGS

FIT MEASURES

Discrepancy

Degrees of freedom

$\mathrm{P}$

Number of parameters

Discrepancy / df

RMR

GFI

Adjusted GFI

Parsimony-adjusted GFI

Normed fit index

Relative fit index

Incremental fit index

Tucker-Lewis index

Comparative fit index

Parsimony ratio

Parsimony-adjusted NFI

Parsimony-adjusted CFI

Noncentrality parameter estimate

NCP lower bound

$\mathrm{NCP}$ upper bound

FMIN

F0

F0 lower bound

F0 upper bound

RMSEA

RMSEA lower bound

RMSEA upper bound

$P$ for test of close fit

Akaike information criterion (AIC

Browne-Cudeck criterion

Bayes information criterion

Consistent AIC

Expected cross validation index

ECVI lower bound

ECVI upper bound

MECVI

Hoelter .05 index

Hoelter .01 index
Model Fit Saturated Independence

1472.79

356

o 54828.563

0

238

4.137

495

0

99

110.765
0.973

0.963

0.979

0.971

0.979

0.719

0.7

0.704

1116.79

1002.15

1238.97

0.691

0.524

0.47

0.582

0.038

0.036

0.04

1

1948.79

1980.65
1188

1267.5
1

0

$1-0$

$1-0$

$\begin{array}{ll}0 & 1\end{array}$

$\begin{array}{ll}0 & 0\end{array}$

0

$\begin{array}{ll}0 & 54333.563\end{array}$

0 53568.153

0 55105.268

$0 \quad 25.741$

0

25.509

25.149

25.871

0.227

0.225

0.229

55026.563

55039.814

$0.915 \quad 0.558$

25.834

$0.861 \quad 0.558$

25.475

$0.972 \quad 0.558$

26.196

$0.93 \quad 0.595$

25.84

590

32

33 
APPENDIX: MODEL FIT INDICES AMOS 4.0

HYPOTHESIS 3: EQUALITY OF INTERCEPTS

FIT MEASURES

Discrepancy

Degrees of freedom

$\mathrm{P}$

Number of parameters

Discrepancy / df

RMR

GFI

\section{Adjusted GFI}

Parsimony-adjusted GFI

Normed fit index

Relative fit index

Incremental fit index

Tucker-Lewis index

Comparative fit index

Parsimony ratio

Parsimony-adjusted NFI

Parsimony-adjusted CFI

Noncentrality parameter estimate

NCP lower bound

NCP upper bound

FMIN

FO

F0 lower bound

F0 upper bound

RMSEA

RMSEA lower bound

RMSEA upper bound

$P$ for test of close fit

Akaike information criterion (AIC

Browne-Cudeck criterion

Bayes information criterion

Consistent AIC

Expected cross validation index

ECVI lower bound

ECVI upper bound

MECVI

Hoelter .05 index

Hoelter .01 index
Model Fit Saturated Independence 3691.05

446

o 54828.563

0

148

8.276
495

0

99

110.765

$\begin{array}{rrr}0.933 & 1 & 0 \\ 0.925 & & 0 \\ 0.94 & 1 & 0 \\ 0.934 & & 0 \\ 0.94 & 1 & 0 \\ 0.901 & 0 & 1 \\ 0.84 & 0 & 0 \\ 0.847 & 0 & 25.741 \\ 3245.05 & 0 & 54333.563 \\ 3054.93 & 0 & 53568.153 \\ 3442.53 & 0 & 55105.268 \\ 1.733 & 0 & 25.509 \\ 1.523 & 0 & 25.149 \\ 1.434 & 0 & 25.871 \\ 1.616 & 0 & 0.227 \\ 0.058 & & 0.225 \\ 0.057 & & 0.229 \\ 0.06 & & \end{array}$

$\begin{array}{rrr}1.872 & 0.558 & 25.834 \\ 1.783 & 0.558 & 25.475 \\ 1.965 & 0.558 & 26.196 \\ 1.881 & 0.595 & 25.84 \\ 297 & & 32 \\ 310 & & 33\end{array}$


APPENDIX: MOOEL FIT INDICES AMOS 4.0

HYPOTHESIS 3 (C): EQUALITY OF INTERCEPTS CONSIDERATION

FIT MEASURES

Discrepancy

Degrees of freedom

$\mathrm{P}$

Number of parameters

Discrepancy / df

RMR

GFI

Adjusted GFI

Parsimony-adjusted GFI

Normed fit index

Relative fit index

Incremental fit index

Tucker-Lewis index

Comparative fit index

Parsimony ratio

Parsimony-adjusted NFI

Parsimony-adjusted CFI

Noncentrality parameter estimate

NCP lower bound

NCP upper bound

FMIN

FO

F0 lower bound

F0 upper bound

RMSEA

RMSEA lower bound

RMSEA upper bound

$P$ for test of close fit

Akaike information criterion (AIr

Browne-Cudeck criterion

Bayes information criterion

Consistent AIC

Expected cross validation index

ECVI lower bound

ECVI upper bound

MECVI

Hoelter 05 index

Hoelter .01 index

$\begin{array}{rrr}\text { Model Fit Saturated Independence } \\ 2872.81 & 0 & 54828.563 \\ 406 & 0 & 495 \\ 0 & & 0 \\ 188 & 594 & 99 \\ 7.076 & & 110.765\end{array}$

0.948

0.936

0.955

0.945

0.955

0.82

0.777

0.783

2466.81

2300.48

2640.55

1.349

1.158

1.08

1.24

0.053

0.052

0.055

0.001

3248.81

3273.98

1188

1267.5

55026.563

55039.814

0.227

0.225

0.229

0

25.509

25.149

25.871

0

54333.563

53568.153

55105.268

1

9


FIT MEASURES

Discrepancy

Degrees of freedom

$\mathrm{P}$

Number of parameters

Discrepancy / df

RMR

GFI

Adjusted GFI

Parsimony-adjusted GFI

Normed fit index

Relative fit index

Incremental fit index

Tucker-Lewis index

Comparative fit index

Parsimony ratio

Parsimony-adjusted NFI

Parsimony-adjusted CFI

Noncentrality parameter estimate

NCP lower bound

NCP upper bound

FMIN

F0

F0 lower bound

F0 upper bound

RMSEA

RMSEA lower bound

RMSEA upper bound

$P$ for test of close fit

Akaike information criterion (AIC

Browne-Cudeck criterion

Bayes information criterion

Consistent AIC

Expected cross validation index

ECVI lower bound

ECVI upper bound

MECVI

Hoelter .05 index

Hoelter .01 index
Model Fit Saturated Independence
2283.31

396

0

198

5.766
0
04828.563

$0 \quad 495$

0

99

110.765

$\begin{array}{rll}0.958 & 1 & 0 \\ 0.948 & & 0 \\ 0.965 & 1 & 0 \\ 0.957 & & 0 \\ 0.965 & 1 & 0 \\ 0.8 & 0 & 1 \\ 0.767 & 0 & 0 \\ 0.772 & 0 & 0\end{array}$

$\begin{array}{lll}1887.31 & 0 & 54333.563\end{array}$

$\begin{array}{lll}1740.76 & 0 & 53568.153\end{array}$

$\begin{array}{lll}2041.31 & 0 & 55105.268\end{array}$

$\begin{array}{lll}1.072 & 0 & 25.741\end{array}$

$\begin{array}{lll}0.886 & 0 & 25.509\end{array}$

$\begin{array}{lll}0.817 & 0 & 25.149\end{array}$

$\begin{array}{lll}0.958 & 0 & 25.871\end{array}$

$0.047 \quad 0.227$

$0.045 \quad 0.225$

$0.049 \quad 0.229$

$0.991 \quad 0$

$1188 \quad 55026.563$

$2705.82 \quad 1267.5 \quad 55039.814$

$\begin{array}{lll}1.258 & 0.558 & 25.834\end{array}$

$\begin{array}{lll}1.189 & 0.558 & 25.475\end{array}$

$\begin{array}{lll}1.33 & 0.558 & 26.196\end{array}$

$\begin{array}{lll}1.27 & 0.595 & 25.84\end{array}$

$424 \quad 32$

$444 \quad 33$ 
APPENDIX: MODEL FIT INDICES AMOS 4.0

HYPOTHESIS 4: EQUALITY OF MEASUREMENT ERROR VARIANCES

FIT MEASURES

Discrepancy

Degrees of freedom

$\mathrm{P}$

Number of parameters

Discrepancy / df

RMR

GFI

Adjusted GFI

Parsimony-adjusted GFI

Normed fit index

Relative fit index

Incremental fit index

Tucker-Lewis index

Comparative fit index

Parsimony ratio

Parsimony-adjusted NFI

Parsimony-adjusted CFI

Noncentrality parameter estimate

NCP lower bound

NCP upper bound

FMIN

F0

F0 lower bound

F0 upper bound

RMSEA

RMSEA lower bound

RMSEA upper bound

$P$ for test of close fit

Akaike information criterion (AI

Browne-Cudeck criterion

Bayes information criterion

Consistent AIC

Expected cross validation index

ECVI lower bound

ECVI upper bound

MECVI

Hoelter .05 index

Hoelter .01 index
Saturated Independence

$\begin{array}{rrr}2633.89 & 0 & 54828.563 \\ 446 & 0 & 495 \\ 0 & & 0 \\ 148 & 594 & 99 \\ 5.906 & & 110.765\end{array}$

$\begin{array}{lll}0.952 & 1 & 0\end{array}$

$0.947 \quad 0$

$\begin{array}{lll}0.96 & 1 & 0\end{array}$

0.955

$\begin{array}{lll}0.96 & 1 & 0\end{array}$

$\begin{array}{lll}0.901 & 0 & 1\end{array}$

$\begin{array}{lll}0.858 & 0 & 0\end{array}$

$0.865 \quad 0 \quad 0$

$\begin{array}{lll}2187.89 & 0 & 54333.563\end{array}$

$\begin{array}{lll}2030.02 & 0 & 53568.153\end{array}$

$\begin{array}{lll}2353.18 & 0 & 55105.268\end{array}$

$\begin{array}{lll}1.237 & 0 & 25.741\end{array}$

$\begin{array}{lll}1.027 & 0 & 25.509\end{array}$

$\begin{array}{lll}0.953 & 0 & 25.149\end{array}$

$\begin{array}{lll}1.105 & 0 & 25.871\end{array}$

$\begin{array}{ll}0.048 & 0.227\end{array}$

$0.046 \quad 0.225$

$\begin{array}{ll}0.05 & 0.229\end{array}$

$0.968 \quad 0$

$\begin{array}{lll}2929.89 & 1188 & 55026.563\end{array}$

$2949.7 \quad 1267.5 \quad 55039.814$

$\begin{array}{rrr}1.376 & 0.558 & 25.834 \\ 1.301 & 0.558 & 25.475 \\ 1.453 & 0.558 & 26.196 \\ 1.385 & 0.595 & 25.84 \\ 412 & & 32 \\ 430 & & 33\end{array}$


APPENDIX: MODEL FIT INDICES AMOS 4.0

HYPOTHESIS 5: EQUALITY OF LATENT MEANS

FIT MEASURES

Discrepancy

Degrees of freedom

$\mathrm{P}$

Number of parameters

Discrepancy / df

RMR

GFI

Adjusted GFI

Parsimony-adjusted GFI

Normed fit index

Relative fit index

Incremental fit index

Tucker-Lewis index

Comparative fit index

Parsimony ratio

Parsimony-adjusted NFI

Parsimony-adjusted CFI

Noncentrality parameter estimate

$\mathrm{NCP}$ lower bound

NCP upper bound

FMIN

F0

F0 lower bound

F0 upper bound

RMSEA

RMSEA lower bound

RMSEA upper bound

$P$ for test of close fit

Akaike information criterion (AIC

Browne-Cudeck criterion

Bayes information criterion

Consistent AIC

Expected cross validation index

ECVI lower bound

ECVI upper bound

MECVI

Hoelter .05 index

Hoelter .01 index
Equality of Means means zero Saturated Independence

$\begin{array}{rrrr}3299.397 & 3691.049 & 0 & 54828.563 \\ 426 & 446 & 0 & 495 \\ 0 & 0 & & 0 \\ 168 & 148 & 594 & 99 \\ 7.745 & 8.276 & & 110.765\end{array}$

$\begin{array}{rrrr}0.94 & 0.933 & 1 & 0 \\ 0.93 & 0.925 & & 0 \\ 0.947 & 0.94 & 1 & 0 \\ 0.939 & 0.934 & & 0 \\ 0.947 & 0.94 & 1 & 0 \\ 0.861 & 0.901 & 0 & 1 \\ 0.809 & 0.84 & 0 & 0 \\ 0.815 & 0.847 & 0 & 0 \\ 2873.397 & 3245.049 & 0 & 54333.563 \\ 2694.255 & 3054.926 & 0 & 53568.153 \\ 3059.909 & 3442.527 & 0 & 55105.268 \\ 1.549 & 1.733 & 0 & 25.741 \\ 1.349 & 1.523 & 0 & 25.509 \\ 1.265 & 1.434 & 0 & 25.149 \\ 1.437 & 1.616 & 0 & 25.871 \\ 0.056 & 0.058 & & 0.227 \\ 0.054 & 0.057 & & 0.225 \\ 0.058 & 0.06 & & 0.229 \\ 0 & 0 & & 0 \\ 3635.397 & 3987.049 & 1188 & 55026.563 \\ 3657.883 & 4006.858 & 1267.5 & 55039.814\end{array}$

1.707

$\begin{array}{ll}1.872 & 0.558\end{array}$

25.834

1.623

$1.783 \quad 0.558$

25.475

1.794

1.965

0.558

26.196

1.717

1.881

0.595

25.84

317

297

310 


\section{APPENDIX}

Dear Manager:

We are asking for your cooperation in completing the attached questionnaire. The questions ask for your opinions about various aspects of your company's human resource management practices. There are no right or wrong answers; we simply ask want your honest opinions. The survey will take approximately 20-30 minutes. This is part of a global study and is likely to help shape future human resource practices.

All of your responses will be kept strictly confidential. Your responses will not be seen by anyone in your company.

We thank you in advance for your participation -- it is greatly appreciated.

Sincerely, 


\section{APPENDIX}

\section{BACKGROUND INFORMATION}

Please place a check $(\square)$ beside or circle the most appropriate answer to each of the following questions. In a few cases please fill-in the blank.

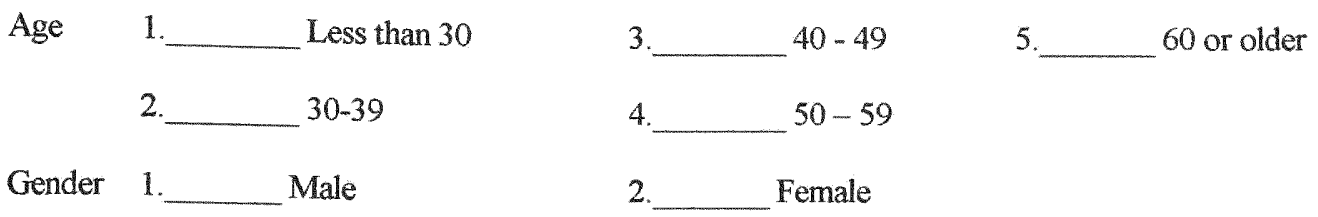

Please indicate the highest grade in school you have completed

$\begin{array}{lll}\text { 1._High School Degree or Less } & 4 . & \text { Some Graduate Education } \\ \text { 2.__ Some College Education } & 5 . & \text { Graduate Degree } \\ \text { 3._Bachelor's Degree } & 6 . & \text { Other }\end{array}$

What is your ethnicity? (e.g. Caucasian, Hispanic, etc.)

Name of Organization

What is your job title?

How long have you been in your current job? years

How long have you been with your current company? years

Do you formally supervise other employees? yes no

If yes, how many employees formally report to you?

How much can you tell by watching the people who report directly to you while they are working?

12

I can tell exactly whether

they are doing their jobs properly or not
45

I can tell very little about how well they are doing their job by watching them

To what extent does your performance depend upon how well others do their job?
1
2
3
4
5
Almost None
A very great deal

What is your current salary?

1.

2.

3.

Less than $\$ 25,000$

$\$ 25,000$ to $\$ 50,000$

$\$ 50,001$ to $\$ 75,000$
4.

5. $\$ 75,001$ to $\$ 100,000$

Greater than $\$ 100,000$ 


\section{APPENDIX}

\section{LEADERSHIP}

How accurately do the following statements describe your company's leadership practices? For each statement provide two responses.

First, use the left column to indicate the extent to which the statements below describe the way Leadership Practices currently are conducted (IS NOW).

Second, use the right column to indicate to what extent the statements below describe the way Leadership Practices ought to be conducted to promote organizational effectiveness (SHOULD BE).

Please use the following scale for the questions below:

$1 \quad 2 \quad 3$

Not at all To a small extent To a moderate extent

My immediate supervisor:

1. Sets specific goals for me to accomplish.

2. Emphasizes high standards of performance.

3. Stresses the importance of work goals.

4. Is friendly and easy to approach.

5. Is eager to recognize and reward good performance.

6. Stresses high standards of performance for group/unit.

7. Is willing to listen to my problems.

8. Treats me with respect.

9. Checks everything; individual judgment is not trusted.

10. When suggestions are made to top management, they receive fair evaluation.
4 5

To a large extent To a very great extent

\begin{tabular}{lllll|lllllll}
\multicolumn{1}{l|}{ IS NOW } & & & \multicolumn{4}{|c}{ SHOULD BE } \\
1 & 2 & 3 & 4 & 5 & 1 & 2 & 3 & 4 & 5 \\
1 & 2 & 3 & 4 & 5 & 1 & 2 & 3 & 4 & 5 \\
1 & 2 & 3 & 4 & 5 & 1 & 2 & 3 & 4 & 5 \\
1 & 2 & 3 & 4 & 5 & 1 & 2 & 3 & 4 & 5 \\
1 & 2 & 3 & 4 & 5 & 1 & 2 & 3 & 4 & 5 \\
1 & 2 & 3 & 4 & 5 & 1 & 2 & 3 & 4 & 5 \\
1 & 2 & 3 & 4 & 5 & 1 & 2 & 3 & 4 & 5 \\
1 & 2 & 3 & 4 & 5 & 1 & 2 & 3 & 4 & 5 \\
1 & 2 & 3 & 4 & 5 & 1 & 2 & 3 & 4 & 5 \\
1 & 2 & 3 & 4 & 5 & 1 & 2 & 3 & 4 & 5
\end{tabular}

Please use the same scale to indicate to what extent your company's leadership practices are effective.

1. The leadership practices help our company to have high-performing employees.

$\begin{array}{lllll}1 & 2 & 3 & 4 & 5\end{array}$

2. The leadership practices help our company

to have employees who are satisfied with their jobs.

$\begin{array}{lllll}1 & 2 & 3 & 4 & 5\end{array}$

3. The leadership practices make a positive contribution to the overall effectiveness of the organization.

$\begin{array}{lllll}1 & 2 & 3 & 4 & 5\end{array}$ 
VITA

\section{ELLEN ANTOINETTE DROST}

1992 MBA, International Business

San Diego State University, College of Business Administration

1989 BS, Finance

San Diego State University, College of Business Administration

ACADEMIC TEACHING EXPERIENCE

2000-current THUNDERBIRD, The American Graduate School of Int'1 Management Visiting Associate Professor, Strategic Management

1998-2000 San Diego State University

1992-1995 International Strategic Management

1996-1998 Florida International University.

International Management and Multinational Strategic Management

1994 -1996 THUNDERBIRD, The American Graduate School of Int'1 Management Guadalajara Summer Program, Global Strategic Management

\section{PUBLICATIONS AND PRESENTATIONS}

Azevedo, A., Drost, E. A. and Mullen, M. (1999). Individualism and Collectivism: Toward a strategy for testing measurement equivalence across cultural diverse groups. Paper presented to the 40th Western Academy of Management, Redondo Beach, CA.

Drost, E. A., Frayne, C. A., Lowe, K. and Geringer, J. M. (2000) Benchmarking training and development practices: a multi-country comparison. Best Paper Proceedings, Southern Academy of Management, Orlando, FL.

Drost, E. A., Frayne, C. A., Lowe, K. and Geringer, J. M. Benchmarking training and development practices: a multi-country comparison. Human Resource Management and The Asia-Pacific Journal of Human Resources. (forthcoming)

Drost, E. A. and Von Glinow, M.A. (1998) Leadership Behaviors in Mexico: Etic philosopies-Emic practices, in Scandura, T.A. \& Serapio, M. G. (Eds.) Research in International Business and International Relations: Leadership and Innovation in Emerging Markets, 7, 3-28. Stamford, CT: JAI Press Inc.

Drost, E. A. and Hofstede, G. (1998). Think locally, act globally. A showcase and tribute to Geert Hofstede presented at the Academy of Management Meetings, San Diego, CA. 
Drost, E. A., Von Glinow, M A and Teagarden M.B. (1996). Human Resource Management in Mexico. Paper presented at the IHRM conference in San Diego.

Drost, E. A., Teagarden, M. B. and Butler, M.C. (1995). International Strategic Human Resource Management: A case study of best practices in Mexico. Paper presented at the 15th International Conference of the Strategic Management Society in Mexico City.

Drost, E. A. (1994). Occupational health and safety activities within the human resource function: a comparison of European best practices. Paper presented at the 4 th Conference on International Human Resource Management. Gold Coast, Australia, July 5-8, 1994.

Drost, E. A. (1994). Government and business relations in Mexico. Paper presented at the Third International Western Academy of Management Conference on Regional and Global Dynamics. Griffith University, Brisbane, Australia, July 10-13, 1994.

Drost, E.A. and Hergert, M. (1993). Jack in the Box goes to Mexico: a case study. CIBER Publication.

Drost, E. A. and Teagarden, M. B. (1993). The function of human resource management in the organization: An empirical study between the United States and Mexico. Paper presented at the 15th International Congress of Training Managers in Mexico City.

Teagarden, M.B., Von Glinow, M.A., Butler, M.C., and Drost, E.A. (1995). The best practices learning curve: Human resource management in Mexico's maquiladora industry. In O. Shenkar (Ed.), Global Perspectives of Human Resource Management, Englewood Cliffs, N.J.: Prentice-Hall, 226-251.

Teagarden, M.B., Von Glinow, M.A., Bowen, D., Frayne, C., Nason, S., Huo, P., Milliman, J., Arias, M., Butler, M., Geringer, J.M., Kim, N., Scullion, H., Lowe, K., and Drost, E. (1995). Toward building a theory of comparative management research methodology: An idiographic case study of the best international human resources management project. Academy of Management Journal, 38(5), 1261-1288.

Von Glinow, M. A. and Drost, E. A. (1998). Coping with process and context in managing a multinational, multicultural, interdisciplinary research consortium. Paper presented at the International Congress of Applied Psychology, San Francisco, CA.

Von Glinow, M. A. and Drost, E. A.(1999). Lessons learned from a 13-country, regional analysis of the IHRM "Best Practices" consortium. A showcase symposium presented at the 1999 Academy of Management meetings, Chicago, IL.

Von Glinow, M.A., Drost, E.A. and Teagarden, M.B. (2000). Converging on international human resource management (IHRM) Practices; Lessons learned for theory and practice. Human Resource Management and the Asia-Pacific Journal of Human Resources. (forthcoming) 